\title{
EMP coupling to ships
}

\author{
F. J. Deadrick, H. S. Cabayan, \\ K. F. Kunz, ${ }^{*}$ R. M. Bevensee, \\ L. C. Martin, and R. W. Egbert
}

Manuscript date: January 1980

Prepared for

Director

Defense Nuclear Agency

Washington, D.C. 20305

*Lu Tech, Inc.

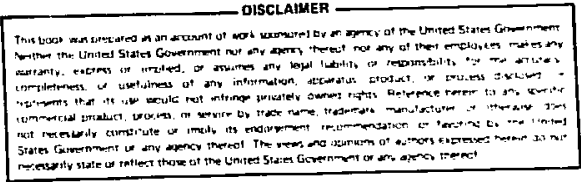

LAWRENCE LIVERMORE LABORATORY University of California • Livermore, California $\bullet 94550$ 
FOREWORD

This research was sponsored by the Defense Nuclear Agency (DNA) under Subtask Code R99QA XEB075 "EMP Interaction and Coupling," work Unit 85 "Coupling Predictions for Ships."

We would like to acknowledge the help and comments of George Baker of DNA and George Brackett, Lloyd Diehl, William Emberson, Charles Simpson, and Neil Stetson of the Naval Surface Weapons Center (NSWC). 
This report makes predictions of the coupling phenomena resulting from the interaction of the electromagnetic pulse (EMP) from a nuclear explosion with the antennas, cables, and mast structural elements of a specific ship, the Canadian ASH ship, HMCs Huron.

Because it is difficult to make predictions of the rather complex conversions of the EMP fields into currents and voltages on the structures and cables of an actual ship, our predictions are based on measurements performed on a 1/48-scale model of the HMCs Huron. These predictions were made without access to the data from FMP simulation tests performed by the Naval Surface Weapons Center (NSWC), Silver Springs, Maryland, on the actual ship.

After we completed our predictions, the Defense Nuclear Agency tasked another organization, Lu Tech, Inc., of Albuquerque, New Mexico, to provide a separate comparison of our predictions with NSWC's measurements from the actual ship.

Dr. Karl Kunz of Lu Tech, Inc. made the comparisons. His results are included in this report as Appendix $A$. 
Foreword

Preface

Abstract

1.0 Introduction

1.1 Báckground

1.2 Organization of the Report

1.3 Overview

1.4 Summary of Predictions

1.5 Sumnary of Scale-Model Measurements and LLL Module Predictions

2.0 Description of the $1 / \triangle 8$-Scale Model HMCs Huron

3.0 Predictions Using the LLL Data Modules

3.1 As-5058 Top Mast Antennas

3.2 HF Transmit Whip Antennas

3.3 HF Receive Fan (Fan "Singlet") Antennas . . . . . . . 3-2

3.4 AN/SRC-23 Flat Top Antennas . . • . . . . . . . . 3-4

3.5 AS-5048 (Port) and AS-5058 (Starb)/AN/WLR/lC Antennas - . . 3-4

3.6 AN/SRN-12 Omega Antenna . . . . . . . . . . . . 3-4

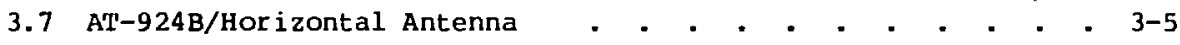

3.8 Mast (Base of one leg) . . . . . . . . . . . . . . 3-5

4.0 LLL 1/48-5cale Model of the HMCs Huron . $_{\text {. }}$. . . . . . . . 4-1

4.1 Equipment Compartment Cable Descriptions $\quad$. . . . . . 4-2

4.1.1 Electronic Warfare Equipment Room (EWER) . . . . . 4-2

4.1.2 Transmitter Room No. $2(\mathrm{TX}-2)$. . . . . . . . . 4-2

4.1.3 Electronic Warfare Control Room (EWCR) . . . . . . 4-2

4.1.4 Combat Control Room (CCR) . . . . . . . . . . 4-3

4.1.5 TíAClN RoOm . . . . . . . . . . . . . . . 4-3

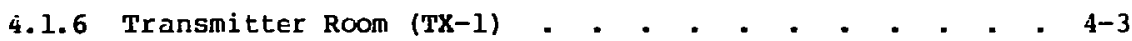

5.0 Scale Model Measurement Techniques and Measured Raw Data . . 5-1

5.1 Impulse Source Fields - . - . . - . . - . - . - 5-1

5.2 Transient Sensors and Probes . . . . . . . . . . . 5-1

5.3 Exterior Coupling . • • . • • • • • • • • • • 5-2

5.4 Interior Coupling Meásurements - Raw Data . . . . . . 5-4

6.0 Processed Data . . . . . . . . . . . . . . . . . 6-1

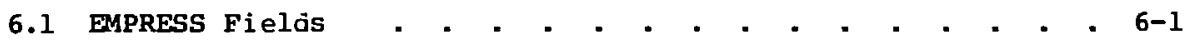


6.2 Data Processing Techniques . . . . . . . . . . . . 5-1

6.3 Full-scale External Coupling Predictions . . . . . . . 6-3

6.4 Full-scale Internal Coupling predictions . . . . . . . 6-3

6.5 Discussion of Measurements . . . . . . . . . . . . 6-4

7.0 Conclusions anã Recommendations . . . . . . . . . . . . 7-1

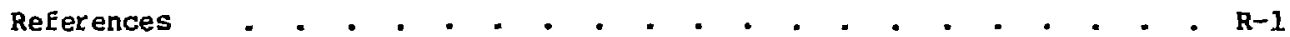

Appenảix: A Comparison of NSWC Measurements and LLL

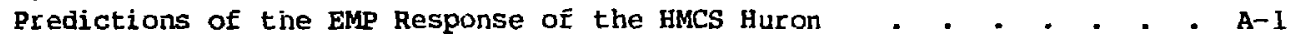

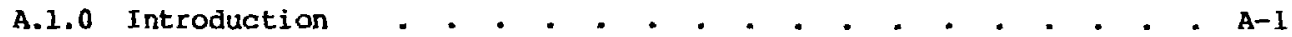

A.2.0 NSWC and LIJ Time-Domain Response Comparisons . . . . . . . A-2

A.2.1 Mast Structural Element Responses . . . - . . - . . . A-2

A.z.2 Interior Cable Responses . . . . . . . . . . . . . A-3

A.2.j Antenna Center Conductor Responses . . . . . . . . . A-7

A.3.0 Mocel Assessment . . . . . . . . . . . . . . . . . A-G

References . . . . . . . . . . . . . . . . . . . . A-33 
1.1. Peaik currents measured on forward portside leg of mast . . 1-6

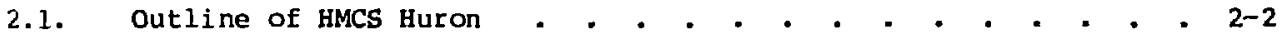

2.2. Upper mast levels . . . . . . . . . • . . . . . 2-3

2.3. Lower mast levels . . . . . . . . . . . . . . . 2-4

2.4. Location of AT-924B antennas . . . . . . . . . . . - 2-5

2.5. Detail of mast cable routings . - . . - . . . . - . 2-6

2.6. Detail of mast cable routings . - . - . - . . . . - 2-7

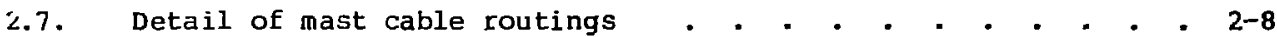

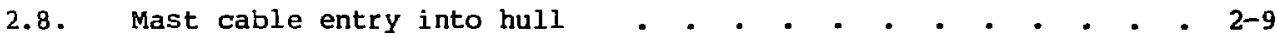

2.9. Exit of cables below cieck . . . . . . . . . . . . . 2-10

2.10. Exit of cables below deck . . . . . . . . . . . . . 2-10

4.1. 1/48-scale model of HMcs Huron on ground plane in front

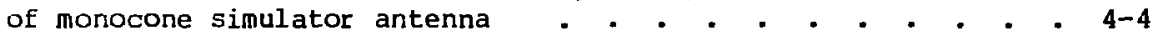

4.2. Antenna locations on 1/48-scale model of HMcs Huron . . . 4-5

4.3a. Cable runs from mast to equipment compartments (02 deck) . . 4-5

4.3b. Cable runs from mast to equipment compartments (01 deck) . 4 4-6

4.3c. Cable runs from mast to equipment compartments (no. 1

deck) . . . . . . . . . . . . . . . . 4-6

5.1. Measured vertical incident field at location of Huron measuremerts . . . . . . . . . . . . . . 5-6

5.2. Frequency spectrum of incident electric field . . . . . . 5-6

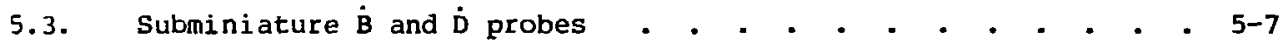

5.4. Tektronix CT-1 current probe - . . . . • . . . . - . 5-8

5.5. Frequency-domain antenna equivalent circuit . . . • • . . 5-8

5.6a. Reflected voltage with antenna feedline open circuited . . . 5-9

5.6b. Reflected voltage with antenna connected to end of

5.6c. Fourier transfor $x_{1}$ of open circuit reflected voltage (Fig. 5.6a) . . . . . . . . . . . . . . . . . 5-10

5.6d. Fourier transform of reflected pulse with antenna
connected to feeciline (Fig. 5.6b) . . . . . . . . . . . . 5-10

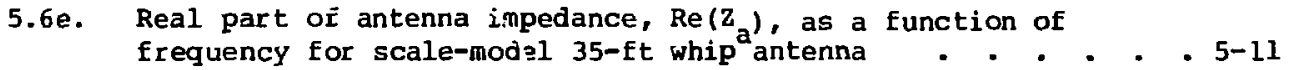

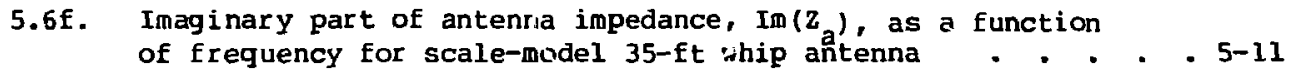

5.6g. Transient response of scale-model antenna due to
incident EM pulse . . . . . . . . . . . . . . . . 5-12 
5.6h. Fourier transform of scale-model transient response - $5-12$

$5.6 i$. Scale model effective height, H of Erequency

(f), as a function $5-13$

5.7a. Real part of antenna input impedance of the MF/HF transmit 35-Et whip (starboard)

5.7b. Imaginary part of antenna input impedance of 35-ft whip (starboard)

5.7c. Effective height of MF/HF transmit 35-ft whip (starboard) . . 5-14

5.8a. Real part of antenna input impedance of $\mathrm{HF}^{\mathrm{a}}$ receive fan (port)

5.8b. Imaginary part of antenna impedance of HF receive Fan (port)

5.8c. Effective height of HF receive fan (port). $5-15$

5.9a. Real part of antenna input impedance of HF receive fan (starboard)

5.9b. Imaginary part of HF receive fan input impedance (starboard)

Effective height of $\mathrm{HF}$ receive $\mathrm{fan}$ (starboard)

5.10a. Real part of $z_{a}$ of the AN/SRN-12 Onega antenna

5.10b. Imaginary part of $z_{a}$ of the AN/SRN-12 Omega antenna

$5-17$

- $5-18$

5.10c. Effective height of the AN/SRN-12 Omega artenna

- 5-18

- $5-19$

5.11a. Real part of $\mathrm{z}_{\mathrm{a}}$ of the $\mathrm{AN} / \mathrm{SRC}-23$ antenna (starboard)

- 5-19

5.11b. Imaginary part of $z$ of the AN/SRC-23 antenna (starboara)

5.11c. Effective height of the AN/SRC-23 antenna (starboard) 5-20

5.12a. Real part of $z_{a}$ of the AN/SRC-23 antenna (port) $5-20$

5.12b. Imaginary part of $\mathrm{z}_{a}$ of the AN/SRC-23 antenna (port) - 5-21

5.12C. Effective height of the AN/SRC-23 (port) 5-21

5.13a. Real part of $z_{a}$ of the AT-924B/AN/SRA-17 antenna (port) $5-22$

5.13b. Imaginary part of $z_{a}$ of the AT-924B/AN/SRA-17c antenna (port)

5.13c. Effective height of the AT-924B/AN/SRA-17C antenna (port)

5.14. Close-up of mast section of Huron model

5.15. Current measurement points on main mast

5.16a. Temporal mast current between levels $A$ and $B$

5.16b. Mast current spectrun botveen levels $A$ and $B$

5.17a. Temporal mast current between levels $C$ and $D$

5.17b. Mast current spectrum between levels $\mathrm{C}$ and $\mathrm{D}$

5.18a - Temporal mast current between levels $G$ and $B$

5.18b. Mast current spectrum between levels $G$ and $H$ 5-23

$5-24$

- 5-25

- 5-26

- 5-26

- 5-27

- 5-27

- 5-28 - 5-28 
5.19a. T'emporal current on horizontal strut at mast level G . . . . 5-29

5.19b. Current spectrum on horizontal strut at mast level G . . . . 5-29

5.20a. EWER bulk-cable current transfer function spectrum . . . . 5-30

5.20b. EWER bulk-cable current . . - . . . . . . . . . . 5-30

5.21a. EWCR bulk-cable current transfer function spectrum . • . . 5-31

5.21b. EWCR bulk-cable current . . . . . . . . . . . . . 5-31

5.22a. 'fACAN bulk-cable current transfer function spectrum . . . . 5-32

5.22b. TACAN bulk-cable current . • • . . . . . . . . . . . 5-32

5.23a. TX-1 julk-cable current transfer function spectrum . • • . 5-33

5.23b. TX-1 bulk-cable current . • • • • • • • • • • • . 5-33

5.24a. TX-2 julk-cable current transfer function spectrum . . . . 5-34

5.24b. TX-2 bulk-cable current . • . • . . . . . . . . . 5-34

5.25a. CCR bulk-cable current transfer function spectrum . • • . . 5-35

5.25b. CCR bulk-cable current . . . . . . . . . . . . . . 5-35

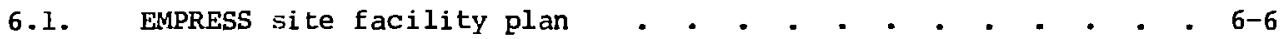

6.2. Vertical electric field produced by EMPRESS simulator

(field measured at ship anchor point) . . * . . . . . . 6-7

6.3. EMPRESS vertical electrį field at ship anchor point . . . . 6-8

6.4. LEL digitized EMPRESS pulse . . . . . . . . . . . . . . 6-9

6.5. Spectrum of EMPREss pulse derived from digitized pulse . . . 6-9

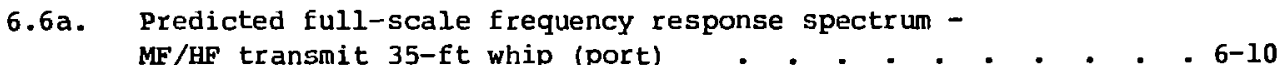

6.6b. Predicted Full-scale transient response - MF/HF

transmit 35-ft whip (port) - . . . . . . . . . . . 6-10

6.6c. Predicted full-scale frequency response spectrum -

6.6d. Predicted full-scale transient response - MF/HF

transmit 35-ft whip (port) . . . . . . . . . . . . 6-11

6.6e. Preäicted full-scale frequency response spectrum -
MF/HF transmit 35-ft whip (port) . $_{\text {. }}$. . . . . . . . 6-12

6.6f. Predicted full-scale transient response - MF/HF
transmit 35-ft whip (port) . . - . . . . . . . . 6-12

6.6g. Predicted full-scale frequency response spectrum -
MF/HF transmit 35-ft whip (port) . . . . . . . . . 6-13

6.6h. Predicted full-scale transient response - MF/HF
transmit 35-ft whip (port) . . . . . . . . . . . 6-13

6.7a. Predicted full-scale frequency response spectrum -
MF/HF transmit 35-ft whip (starboard) . . . . . . . . . . 6-14 
6.7b. Prodicted full-scale transient response - MF/HF

transmit 35-ft whip (starboard) . . . . . . . . . . . 6-14

6.7c. Predicted full-scale frequency response spectrum MF/HF transmit 35-ft whip (starboard)

6-15

6.7d. Predicted full-scale transient response - MF/HF

transmit 35-ft whip (starboard) . . . . . . . . . . . 6-15

6.7e. Predicted full-scale frequency response spectrum MF/HF transmit 35-ft whip (starboard) . . . . . . . . . 6-16

6.7f. Predicted full-scale transient response - MF/HF

transmit 35-ft whip (starboard) . . . . . . . . . . . 6-16

6.7g. Predicted full-scale frequency response spectrum -

MF/HF transmit 35-ft whip (starboard) . . . . . . . . . 6-I7

6.7h. Preaicted full-scale transient resf unse - MF/HF

transmit 35-ft whip (starboard) - . . . . . . . . . . 5-17

6.8a. Predicted full-scale frequency response spectrum - HF receive fan (port)

6.8b. Predicted full-scale tuansient response - HF receive fan (port) - . . . . . . . . . . . . . . . . 6-18

6.8c. Predicted full-scale frequency response spectrum - HF receive fan (port)

6.8d. Predicted full-scale transient response - HF receive fan (port) . . . . . . . . . . . . . . . . . . 6-19

6.8e. Predicted full-scale frequency response spectrum - HF receive fan (por:)

6.8E. Predicted full-scale transient response - HF receive fan (port) . . . . . . . . . . . . . . . . . . 6-20

6.8g. Predicted full-scale frequency response spectrum - HF receive fan (port)

6.8h. Predicted full-scale transient response - HF receive

fan (port) . . . . . . . . . . . . . . . . . . 6-21

6.9a. Predicted full-scale frequency response spectrum - HF receive fan (staiboard)

6.9b. Predicted full-scale transient response - HF receive fan (starboard)

6.9c. Predicted full-scale frequency response spectrum - HF receive fan (starboard)

6.9d. Predicted full-scale transient response - HF receive fan (starboaró:

6.9e. Predicted full-scale frequency response spectrum - HF receive fan (starboard)

6.9f. Predicted full-scale transient response - HF receive fan (starboard)

6.9g. Predicted full-scale frequency response spectrum - HF receive fan (starboard) 
6.9h. Predicted full-scale transient response - HF receive Fan (starboard)

6.10a. Predicted full-scale frequency response spectrum AN/SRC-23 Flat top (port)

6.10b. Predicted full-scale transient response - AN/SRC- $s$ tlat top (port)

6.10c. Predicted full-scale frequency response spectrum AN/SRC-23 Elat top (port)

6.10d. Predicted full-scale transient response - AN/SRC-23

flat top (port)

6.10e. Predicted full-scale frequency response spectrum AN/SRC-23 flat top (port)

6.10f. Predicted full-scale transient response - AN/SRC-23 flat top (port)

6.10g. Predicted full-scale frequency response spectrum AN/SRC-23 flat top (port) . . . . . . . . . . . . . 6-29

6.10h. Predicted full-scale transient response - AN/SRC-23 Elat top (port)

5.lla. Predicted full-scale frequency response spectrum AN/SRC-23 flat top (starboard) $6-29$

6.11b. Predicted full-scale transient response - AN/SRC-23 flat top (starboard)

6.1lc. Predicted full-scale frequency response spectrum AN/SRC-23 flat top (starboard)

6.1ld. Predicted full-scale transient response - AN/SRC-23 flat top (starboard)

6.lle. Predicted full-scale frequency response spectrum AN/SRC-23 flat top (starboard)

5.llf. Predicted full-scale transient response - AN/SRC-23 Elat top (starboard)

6.11g. Predicted full-scale frequency response spectrum AN/SRC-23 flat top (starboard)

6.11h. Predicted full-scale transient response - AN/SRC-23 flat top (starboard)

6.12a Predicted full-scale Erequency response spectrum AN/SRN-12 Omega

6.12b. Predicted full-scale transient response - AN/SRN-12 Omega

6.12c Predicted full-scale frequency response spectrum AN/SRN-12 Omega

6.12d. Predicted full-scale transient response - AN/SRN-12 Onega

6.12e Predicted full-scale frequency response spectrum AN/SRN-12 Omega 
6.12f. Predicted full-scale transient response - AN/SRF-12

Omega

6.12g Predicted full-scale frequency response spectrum -

AN/SRN-12 Omega

6.12h. Predicted full-scale transient response - AN/SRN-12 Omega

5.13a. Predicted full-scale frequency response spectrum AT-924B/AN/SRA-17C (port)

6.13b. Predicted full-scale transiont response AT-924B/AN/SRA-17C (port)

6.13c. Predicted full-scale frequency response spectrum AT-924B/AN/SRA-17C (port,

6.13d. Predicted full-scale transient response AT--924B/AN/SRA-17C (por :)

6.13e. Predicted full-scale frequency response spectrun AT-924B/AN/SRA-17C (port)

6.13f. Predicted full-scale transient response AT-924B/AN/SRA-17C (port)

6.13g. Predicted full-scale frequency response spectrum AT-924B/AN/SRA-17C (port)

6.13h. Predicted full-scele transient response AT-924B/AN/SRA-17C (port)

6.14a. Predicted full-scale mast current spectrum between levels $A$ and $B$ (forward port)

6.14b. Predicted full-scale transient mast surrent between levels $A$ and $B$ (forward port) $6-42$

6.15a. Predicted full-scale mast current spectrum between levels $C$ and $D$ (forward port) $6-43$

6.15b. Predicted full-scale mast current between levels $c$ and D (forward port)

6.16a. Predicted full-scale mast current spectrum betiveen levels $\mathrm{G}$ and $\mathrm{H}$

6.16b. Predicted full-scale mast current between levels $G$ and $H \quad$. 6-44

6.17a. Predicted full-scale mast current spectrum on horizontal strut at level $\mathbf{G}$

6.17b. Predicted full-scale mast current on horizontal strut at level $G$

6.18a. Predicted full-scale current spectrum on EWER cable - 6-45

6.18b. Predicted full-scale transient current on EWER cable - 6-46

6.19a. Predicted full-scale current spectrum on 5WCR cable - 6-46

6.19b. Predicted full-scale transient current on EWCR cable - 6-47

6.20a. Predicted fuil-scale current spectrum on TACAN room . 6-47 cable 
6.20b. Predicted full-scale transient current on TACAN room cable

6.2la. Predicted full-scale curcent spectrum on TX-1 room cable . . 6-49

6.21b. Predicted full-scale transient current on $\mathrm{TX}-1$ room cable

6.22a. Predicted full-scale current spectrum on TX-2 room cable , 6-50

6.22b. Predicted full-scale transient current on $\mathrm{TX}-2$ room cable

6.23a. Predicted full-scale current spectrum on CCR cable . . . . 6-51

6.23b. Predicted full-scale transient current on CCR cable . . . . 6-5l

A.l. Full-scale current on horizontal mast element at level G . . . . . . . . . . . . . . . . . . . A-ll

A.2. Full-scale current frequency spectrum on horizontal mast element at level $\mathbf{G}$

A.3. Full-scale current on mast between levels $\mathbf{G}$ and $\mathbf{H}$. . . . . A-13

A.4. Full-scale current frequency spectrum on mast between levels

$\mathrm{G}$ and $\mathrm{H}$. . . . . . . . . . . . . . . . . . . A-14

A.5. Full-scale current on mast between levels $C$ and $D$ (forward port side)

A.6. Full-scale current frequency spectrum on mast between levels $\mathrm{C}$ and $D$ (forward port side)

A.7. Full-scale current on mast between levels $A$ and $B$ (forward port side)

A.8. Full-scale current spectrum on mast between levels $A$ and $B$ (forward port sicle)

A.9. Full-scale bulk cable current (TACAN room) . . . . . . . A-19

A.10. Full-scale bulk cable current spectrum (TACAN room) . . . . A-20

A.11. Full-scale bulk cable current (EWCR room) . . . . . . . A-2l

A.12. Full-scale bulk cable current spectrum (EWCR room) . . . . A-22

A.13. Full-scale open-circuit voltage for 35-ft whip antenna

(port) . . . . . . . . . . . . . . . . . . . A-23

A.14. Full-scale open-circuit voltage spectrum for 35-ft whip antenna (port)

A.15. Full-scale short-circuit current for 35-ft whip antenna (port)

A.16. Full-scale short circuit current spectrum for 35 -ft whip antenna (port)

A.17. Full-scale open-circuit voltage for lower fan antenna (starboard)

A.18. Full-scale open-circuit voltage spectrum for lower fan antenna (starboard)

A.19. Full-scale short-circuit current for lower fan antenna (star board) 
A.20. Full-scale short-circuit current spectrum for lower fan antenna (starboard) . . . . . . . . . . . . . . A-30

A.21. Full-scale current for horizontal whip (port side of mast) - . . . . . . . . . . . . . . . . . A-31

A.22. Full-scale current spectrum for horizontal whip (port side of mast) . . . . . . . . . . . . . . . . A-32

xiv 
1. I Full-scale peak time-domain quantities referenced to EMPRESS incicient field . . . . . . . . . . . . . . 1-3

1.2 Estimated peak time-domain responses of the antennas on the Huron - . - . - . . - . - . . . - . 1-4

1.3 Scaled-up peak bulk-cable currents measured on cables interior to Huron . . . . . . . . . . . . . . 1-5

A.1 Mast structural element data measurement locations . . . . . A-3

A.2 Mast structural element data comparisons . . . . . . . - . A-4

A. 3 NSWC's assessment of the agreement between the model and full-scale measurements with respect to the interior cables . . . . . . . . . . . . . . . A-5

A. 1 Interior cable data comparisons . . . . . . . . . . . . A-7

A.5 NSWC antenna measurement observations . . - . - . . . . - . A-8

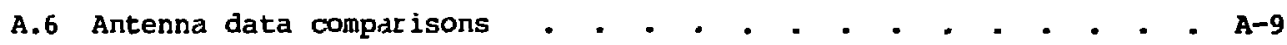


ABSTRACT

Scale-model tests were conducted to establish the adequacy and limitations of model measurements as tools for predicting electromagnetic pulse (ENP) coupling voltages and currents to the critical antennas, cables, and metallic structures on ships. The scale-model predictions are compared with the results of the full-scale EMP simulation test of the Canadian ASA ship, Hucs Huron. (The EAP coupling predictions in this report were made without prior knowledge of the results of the data from the Hucs Huron tests.)

This report establishes that the scale-model tests in conjunction with the data base from our Eup coupling modules* provides the necessary information for source model development and permits effective, low cost study of particular system configurations.

\subsection{INTRODUCTION}

\section{I.1 BACKGROUND}

The Engineering Research Division at Lawrence Livermore Laboratory (LLH), while under contract to the Defense Nuclear Agency (DNA), developed tools to predict the results of electromagnetic pulse (EXP) coupling to a variety of military electronic systems. These tools, which are based primarily on scale and numerical modeling, resulted in a number of useful observations and guidelines which are formulated in an earlier report, External Coupling of BrP to Generic System Structures. 1 Section 10 of the referenced report uses test data from sevaral system tests to validate the tools. At the time the report was published, the predictive capabilities of scale modeling had not been used as a pretest tool.

At the beginning of 1978, George Baker of DNA, requested that Lil reorient its efforts to more closely parallel work being done at various service

Hodule in this context denotes a self-contained package of information about the $\operatorname{ExP}$ response of a generic class of structures. 
laboratories. In particular, William Emberson of the Naval Surface Weapons Center (NSWC), who was in the midst of planning the testing of the Canadian ASW ship, HMCS Huron, at the EMPRESS facility, needed LLL's services. Emberson provided a required list of calculations which was later updated by Charles $\mathbf{G}$. Simpson of DNA.

Although it would have been useful to examine antenna placements and cable runs both outside and inside the hull, by the time LIJ began its work on the project the ship had left NSWC, and it was impractical to set up a visit for a visual inspection.

George Brackett and Neil stetson, who were involved in the tests at the EMPRESS facility, provided us with the necessary engineering information that was not available from photographs, engineering drawings, and sketches.

\subsection{ORGANIZAT'ION OF THE REPORT}

Section 1 gives an overview and summary of the predictions based on scale models. Section 2 describes the important structural features of the Huron as determined by NSWC and shows photographs and sketches of the Huron's various compartments. Section 3 describes the H.L data module predictions. Section 4 describes the LLL 1/48-scale model of the Huron. Section 5 gives the data from the scale model tests. Section 6 gives the processed data extrapolated to the EMPRESS field as required by NSWC. Section 7 gives the conclusions and a discussion of the data obtained during the tests: The Appendix gives the results of our 1/48-scale predictions with NSWC's full-scale measurements.

\subsection{OVERVIEW}

Our predictions are based on both the data from the LLL modules and the scale-model tests. To the best of our knowledge, this is the first attempt to make extensive internal coupling predictions for ships based on scale-model tests.

The LLL modules ${ }^{1}$ give data for estimating the EMP response of various antenna configurations. Before we conducted the scale-model tests, we used the data in the modules to predict the peak voltages and currents on all metallic collectors exterior to the hull. (See Table 1.1.) 
TABLE 1.1. Full-scale peak time-domain quantities referenced to EMPRESS incident field.

\begin{tabular}{|c|c|c|c|c|c|}
\hline $\begin{array}{l}\text { Antenna type } \\
\text { and location }\end{array}$ & $\begin{array}{c}\mathbf{v}_{\text {50-ohm }} \\
\text { load, } \\
\text { kv }\end{array}$ & $\begin{array}{c}I_{50-o h m}{ }^{a} \\
\text { load, } \\
\text { A }\end{array}$ & $\begin{array}{c}\text { open }^{a} \\
\text { circuit, } \\
\text { kV }\end{array}$ & $\begin{array}{c}I_{\text {short }} \\
\text { circuit } \\
\text { kV }\end{array}$ & \\
\hline $\begin{array}{l}\text { MF/AF Tx } \\
\text { 35-ft whip (Port) }\end{array}$ & 3.9 & $78.0 \quad(97)$ & $21.3(33)$ & 90.0 & $(130)$ \\
\hline $\begin{array}{l}\text { MF/AF Tx } \\
\text { 35-ft Whip (Stbd) }\end{array}$ & 3.4 & 68.0 & 20.0 & 78.0 & \\
\hline $\begin{array}{l}\text { HF receive } \\
\text { fan (Port) }\end{array}$ & 1.7 & $34.0(2.8)$ & $8.2(0.91)$ & 46.0 & $(3.5)$ \\
\hline $\begin{array}{l}\text { HF receive } \\
\text { fan (Stbd) }\end{array}$ & 0.37 & 7.4 & 1.24 & 11.5 & \\
\hline $\begin{array}{l}\text { AN/SRC- } 23 \\
\text { flat top (Port) }\end{array}$ & 7.1 & 142.0 (48) & $10.8(12)$ & 175.0 & (65) \\
\hline $\begin{array}{l}\text { AN/SRC- } 23 \\
\text { flat top (Stbd) }\end{array}$ & 2.6 & 52.0 & 10.4 & 58.0 & \\
\hline AN/SRN-12 Omega & 0.475 & $9.5(8.1)$ & $2.7(3.2)$ & 11.5 & $(10.1)$ \\
\hline $\begin{array}{l}\text { AT-924B/An/SRA-17C } \\
\text { (Port) }\end{array}$ & 0.255 & $5.1(0.47)$ & $5.1(0.14)$ & 5.7 & $(0.75)$ \\
\hline
\end{tabular}

Numbers shown in parentheses are the LLL data module predictions.

To a certain extent, validation for external coupling (i.e. to antennas and other metallic structures outside the hull) has been established (see sec. 10 of Ref. 1); therefore, good agreement is expected in this area. However, no such claim can be made for internal coupling predictions i.e., for cables and other metallic structures inside the hull.

Because hull and cable shielding cannot be evaluated, and because secund order effects, such as coupling through cracks or undetected openings are not modeled, the limitations on making internal coupling predictions are obvious. However, if these limitations are not critical, and if the comparisons are accurate enough to be useful to system designers and hardening engineers, then scale models will be a useful tool for EMP coupling assessment of ships.

We caution that scale-model tests should not be considered as a replacement for system tests; at best, they can serve as pretest tools or as complements to other tests. 


\subsection{SUMMARY OF PREDICTIONS}

Table 1.? summarizes the estimated peak time-domain responses of various antennas on the Huron induced by the vertically-polarized mode of the EMPRESS facility. The open-circuit voltage, the 50-ohm current, and the stort-circuit current at the load terminals, where the antenna connects to its cable, are listed for antennas on the port side (the side nearest the sinulator). The responses were predicted from the EMP data modules (see Sec. 3).

\subsection{SUMMARY OF SCALE-MODEI, MEASUREMENTS AND LLL MODULE PREDICTIONS}

In all cases we scaled up the model measurements by the model size-factor of 48 and convolved our model measurements with the vertically-polarized pulse from the EMPRESS facility; this procedure gave data directly comparable with NSWC's full-scale measurements. The 50-ohm load voltage and currents, the open-circuit voltage, and the short-circuit current at the load terminals (where the antenna connects to its cable) are all obtainable from full-scale

TABLE 1.2. Estimated peak time-domain responses of the antennas on the Buron.

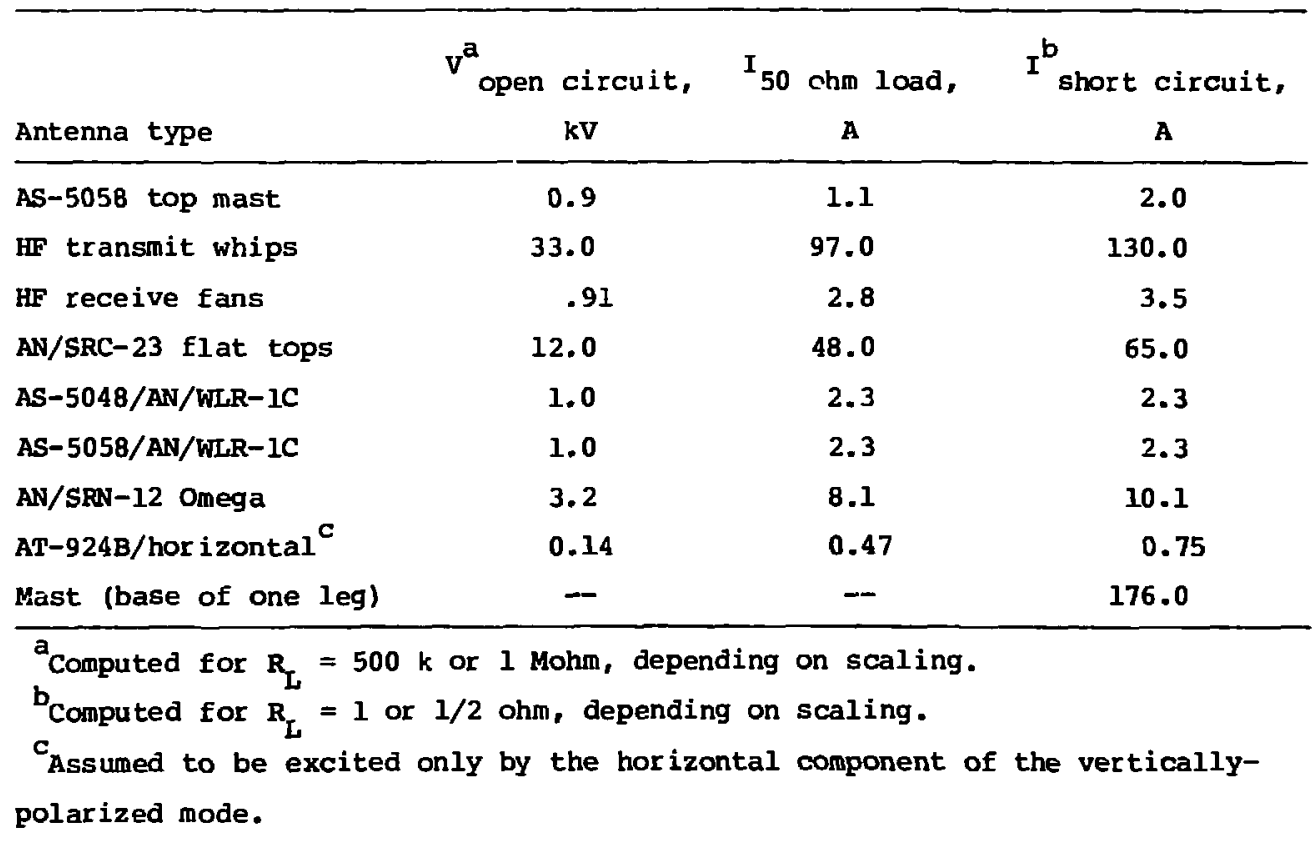


measurements. Table 1.2 gives the full-scale measurement data of the antennas on board the Huron.

Figure 1.1 shows the various antennas tested and lists the peak currents detected. We used a small current probe to test for current on various members of the Huron's main mast. Again, the scale-faodel measurements were scaled-up to the full-size EMPREss level for comparison.

We measured the bulk-cable currents inside the Huron's superstructure. These bulk-cable currents are, for example, the cable shield currents on the cables running from the mast antennas down to the various compartments. The results of these measurements are irstallation dependent. We modeled the full-scale ship without knowledge of groundings, cable layouts, cracks and gaps, etc. The peak cable-current measirements give a ball-park estimate for systems assessment. Table 1.3 lists the internal bulk-cable currents scaled to a full-sized ship illuminated by a vertically-polarized EMPRESS type pulse. Because the EMP coupling modules do not cover internal coupling problems. no internal coupling predictions were made.

TABLE 1.3. Scaled-up peak bulk-cable currents measured on cables interior to Huron. a

Cable

Location

EWER

EWCR

TACAN roOm

$T X-1$ room

TX-2 room

CCR
Internal coupling configuration

Wire terminated to horizontal strut at mast level $\mathrm{J}$. (20-wire bundle to fiberglass mast also in room.)

Open wire to top of fiberglass mast (open circuited). (10-wire bundle also in room.)

Wire from TACAN antenna at mast level $J$. through 01 deck.

wire connected to port flat top through 01 deck, to TACAN room.

single wire from top of mast (open end). through ENER

Wire grounded to horizontal strut at mast level J (AS-5041/AN/SRC 503) through

01 deck to EWCR. (10-wire bundle to TACAN)
Peak bulk current, A 38

24 38 7

5.4

\footnotetext{
${ }^{a}$ Currents referenced to EMPRESS pulse.
} 


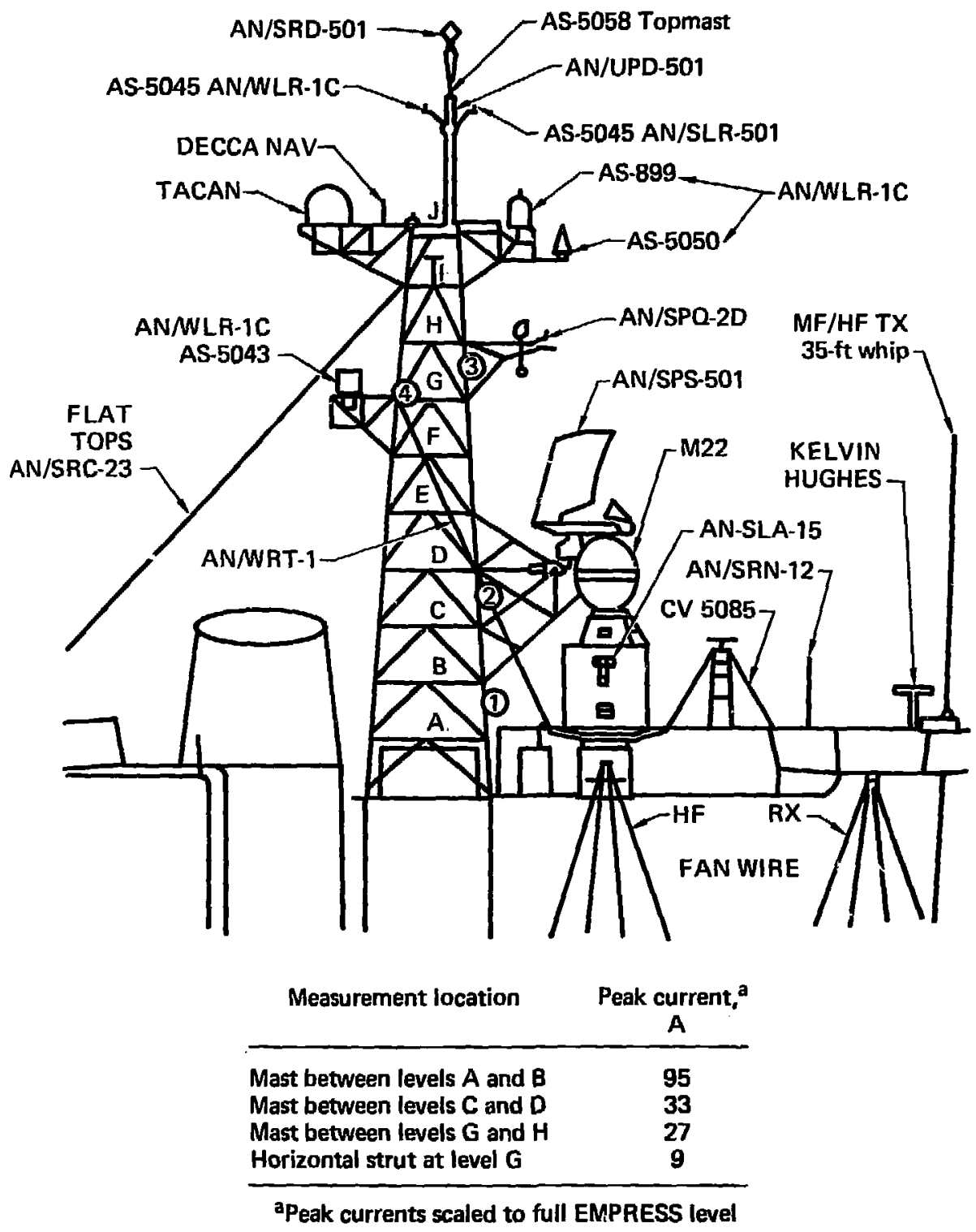

FIG. 1.1. Peak currents measured on forward portside leg of mast. 


\subsection{DESCRIPTION OF THE 1/48-SCALE MODEL HMCS HURON}

Our model was based on photographic information and on engineering data provided by NSWC personnel. Figure 2.1 shows the relevant dimensions and the various decks and compartments that were modeied for the interior coupling measurements. Figures 2.2 through 2.5 show the details of the main mast and cable routings down the mast. Figures 2.6 through 2.10 show additional features of the ship relevant to the study.

Actual details of the cable runs, grounds, connections, and terminations were not available or unknown. Although some of these details could have been obtained by an on-site inspection of the ship, they would have been difficult to resolve. This is particularly true of cable shields, where the shield may be grounded to the mast or hull at several points or left floating. 


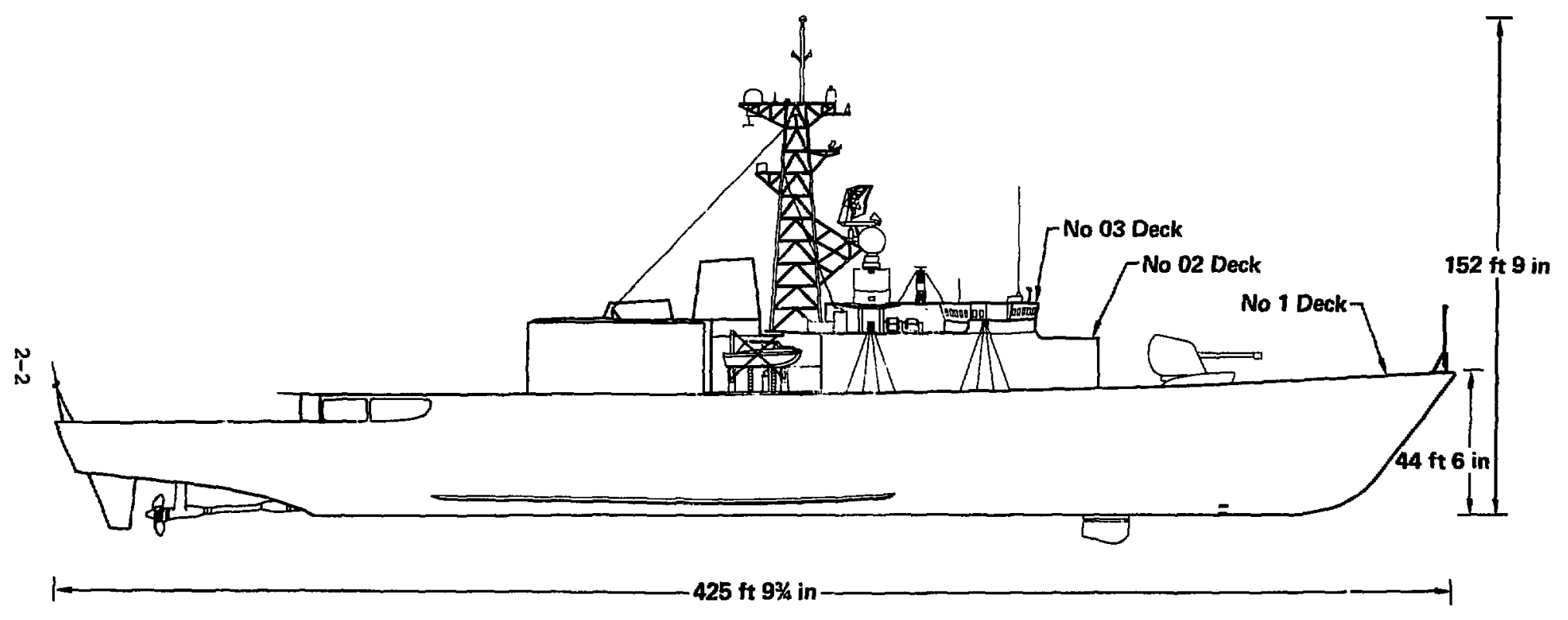

FIG. 2.1. Outline of HMCS Huron. 

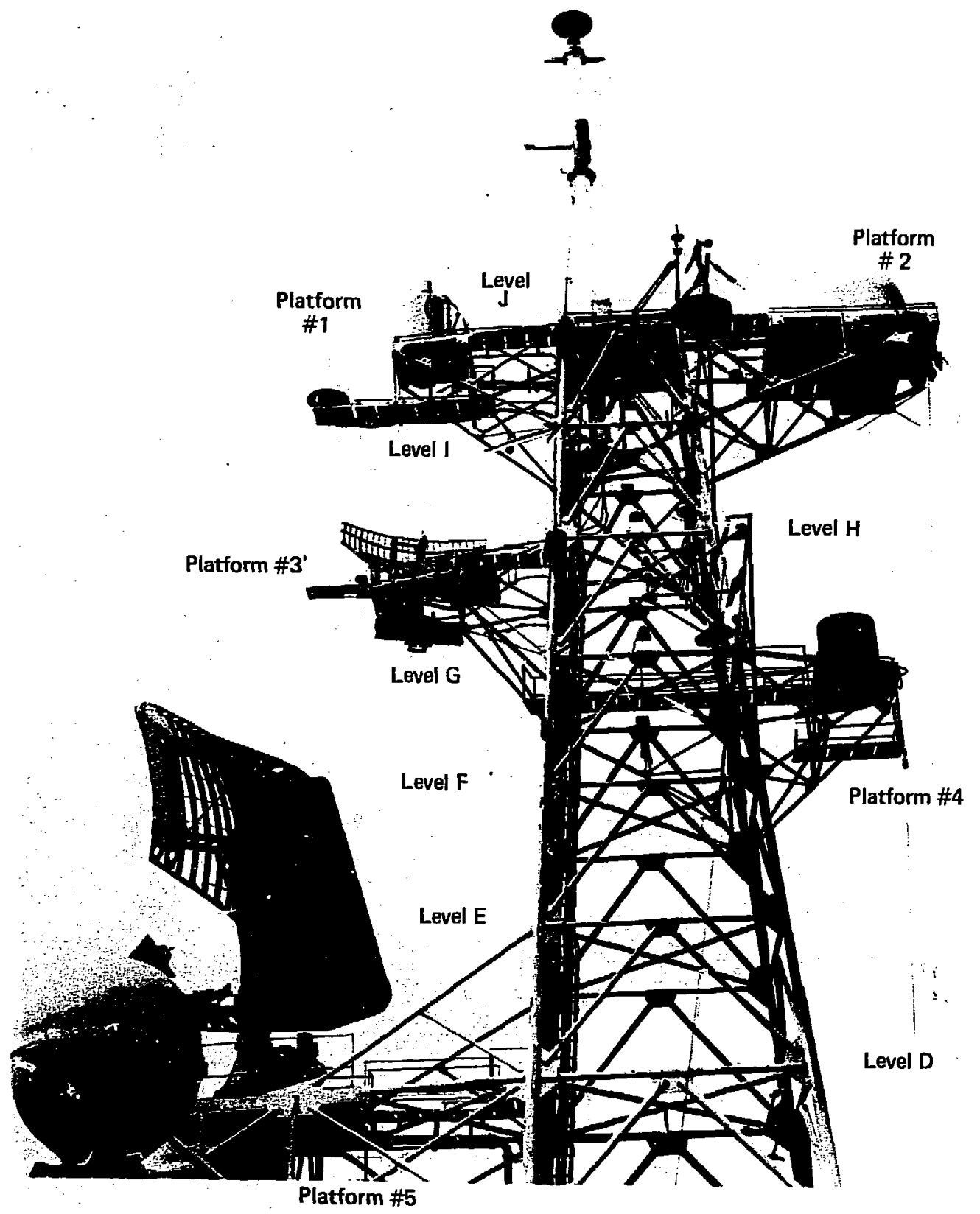

FIG. 2.2. Upper mast levels. 


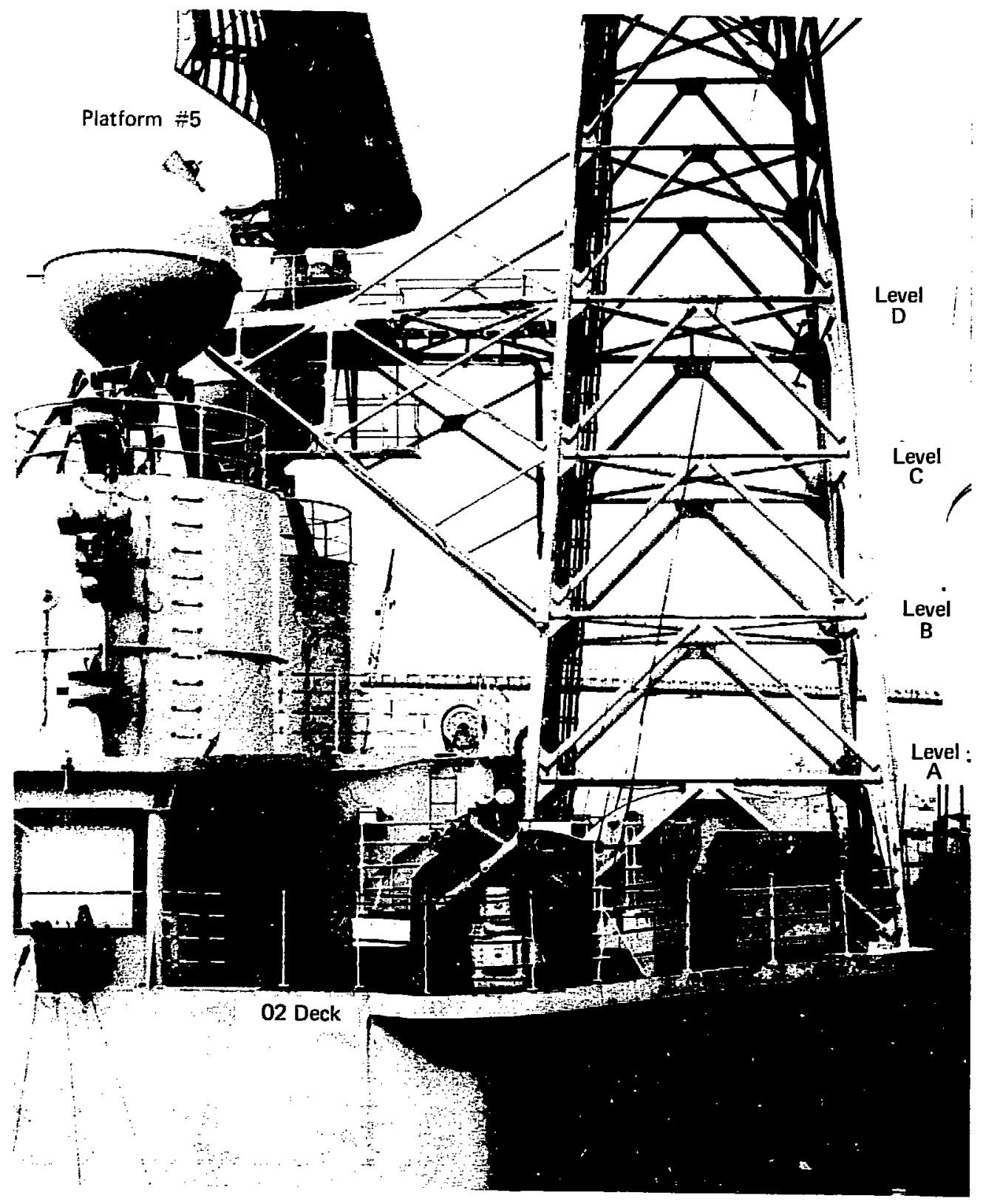

FIG. 2.3. Lower mast levels. 


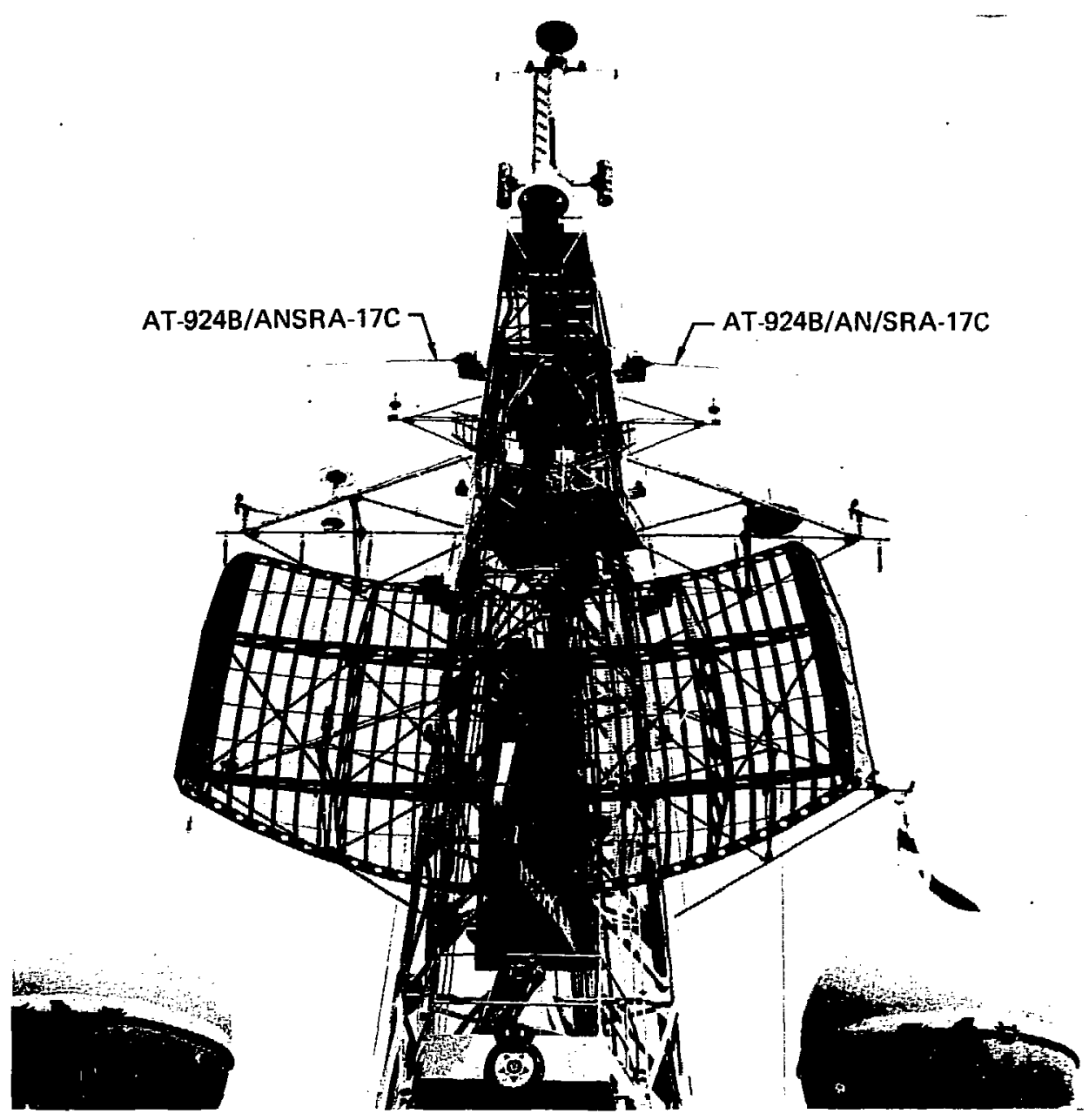

FIG. 2.4. Location of AT-924B antennas. 


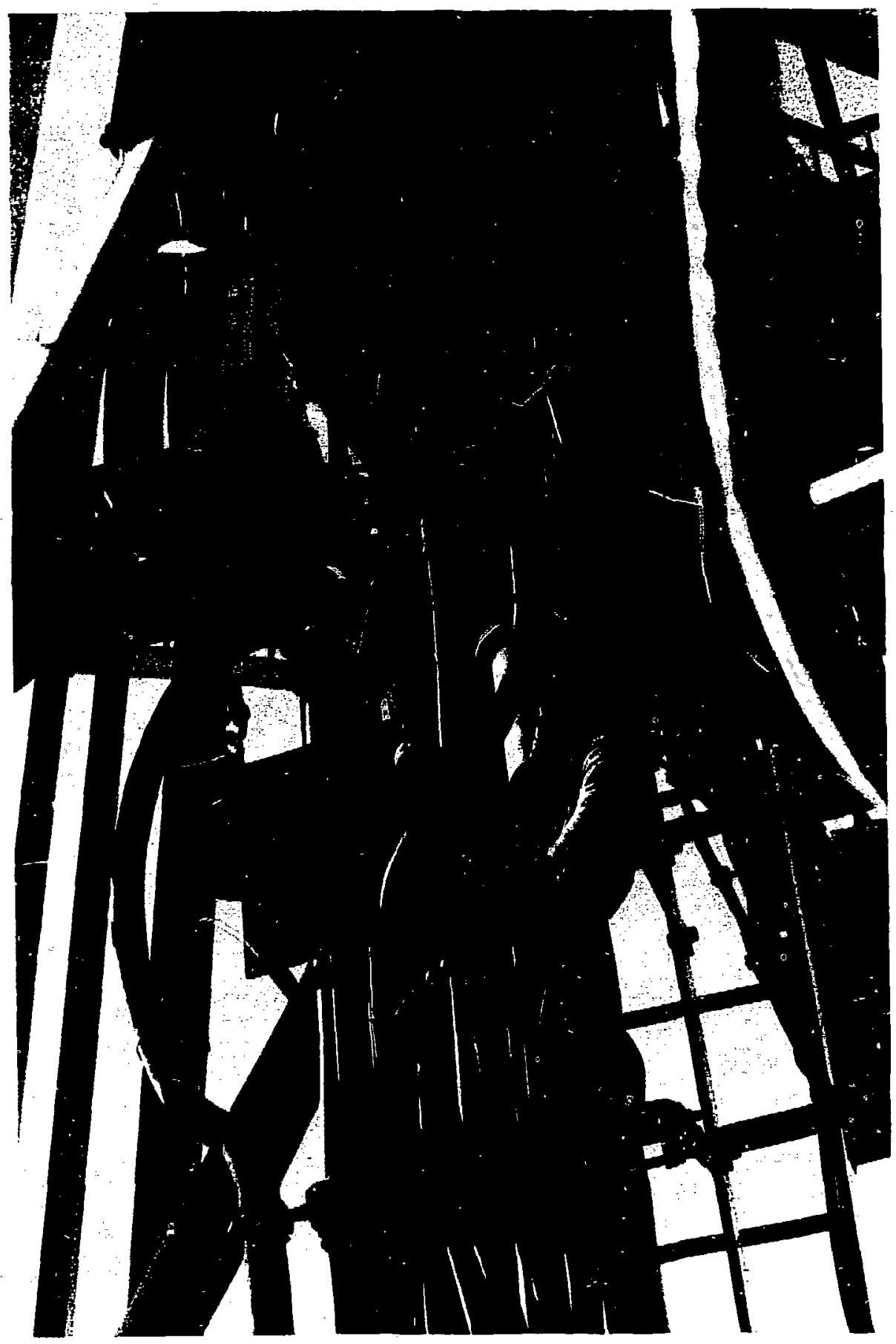

FIG. 2.5. Detail of mast cable coutings. 


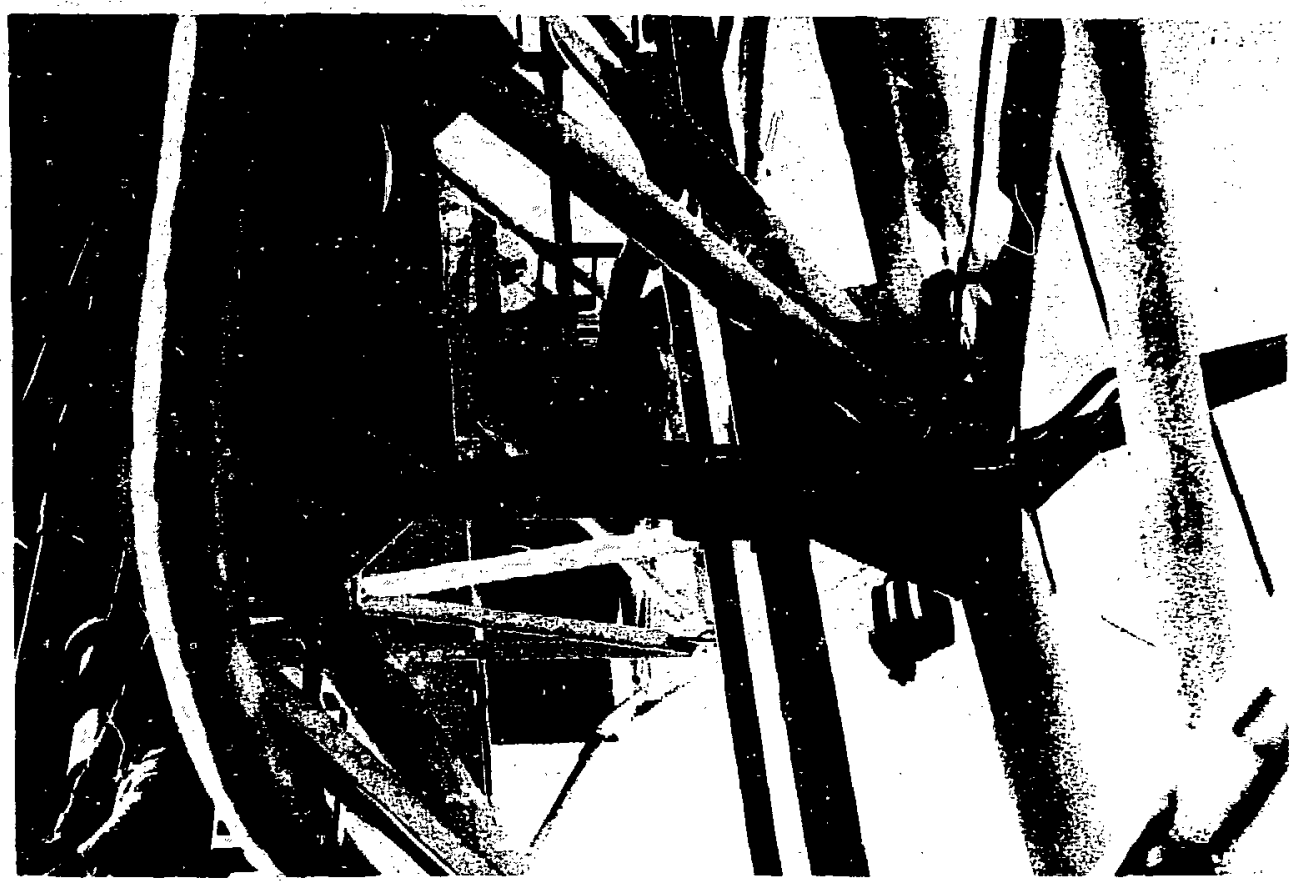

FIG. 2.6. Detail of mast cable routings. 


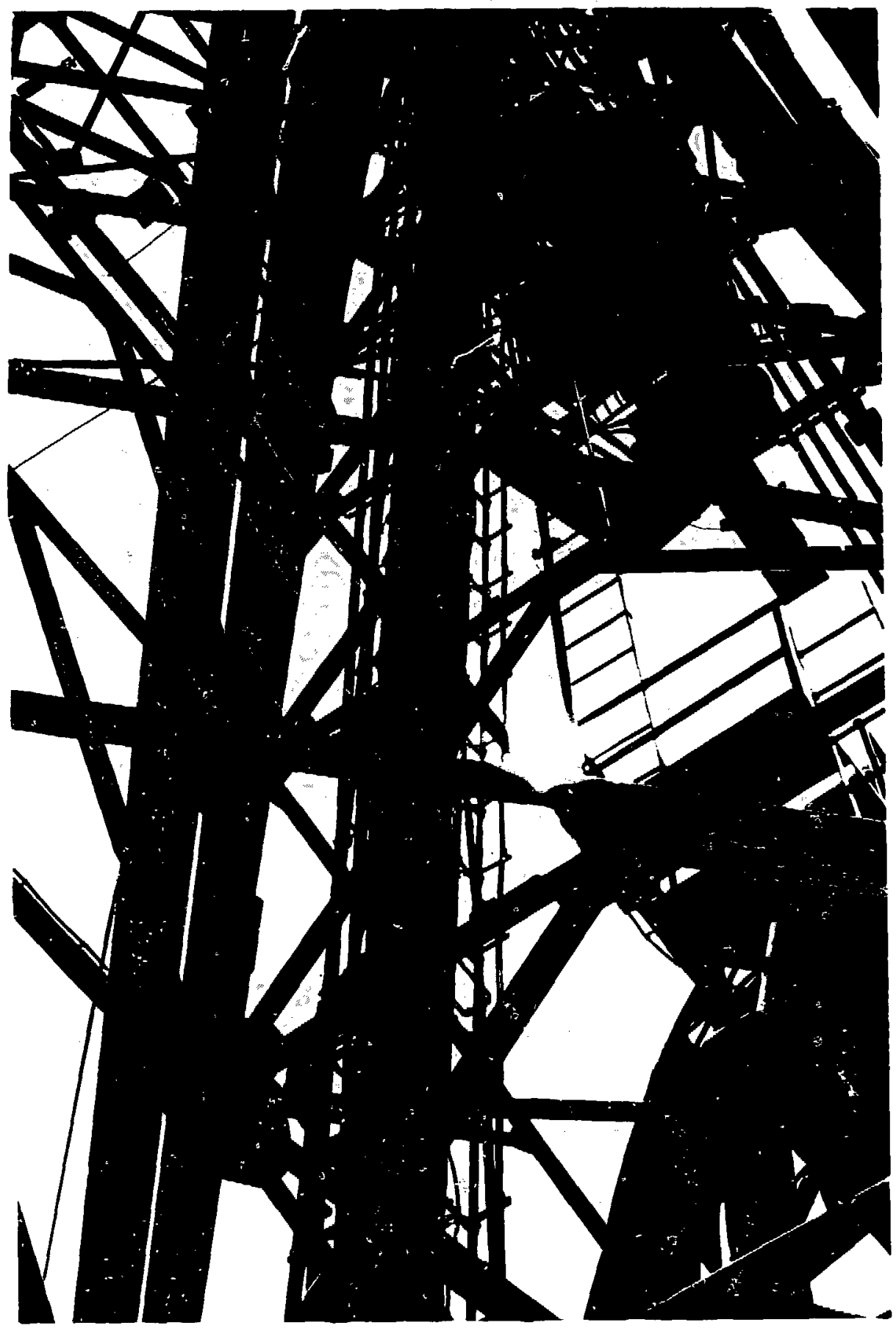

FIG. 2.7. Detail of mast cable routings. 


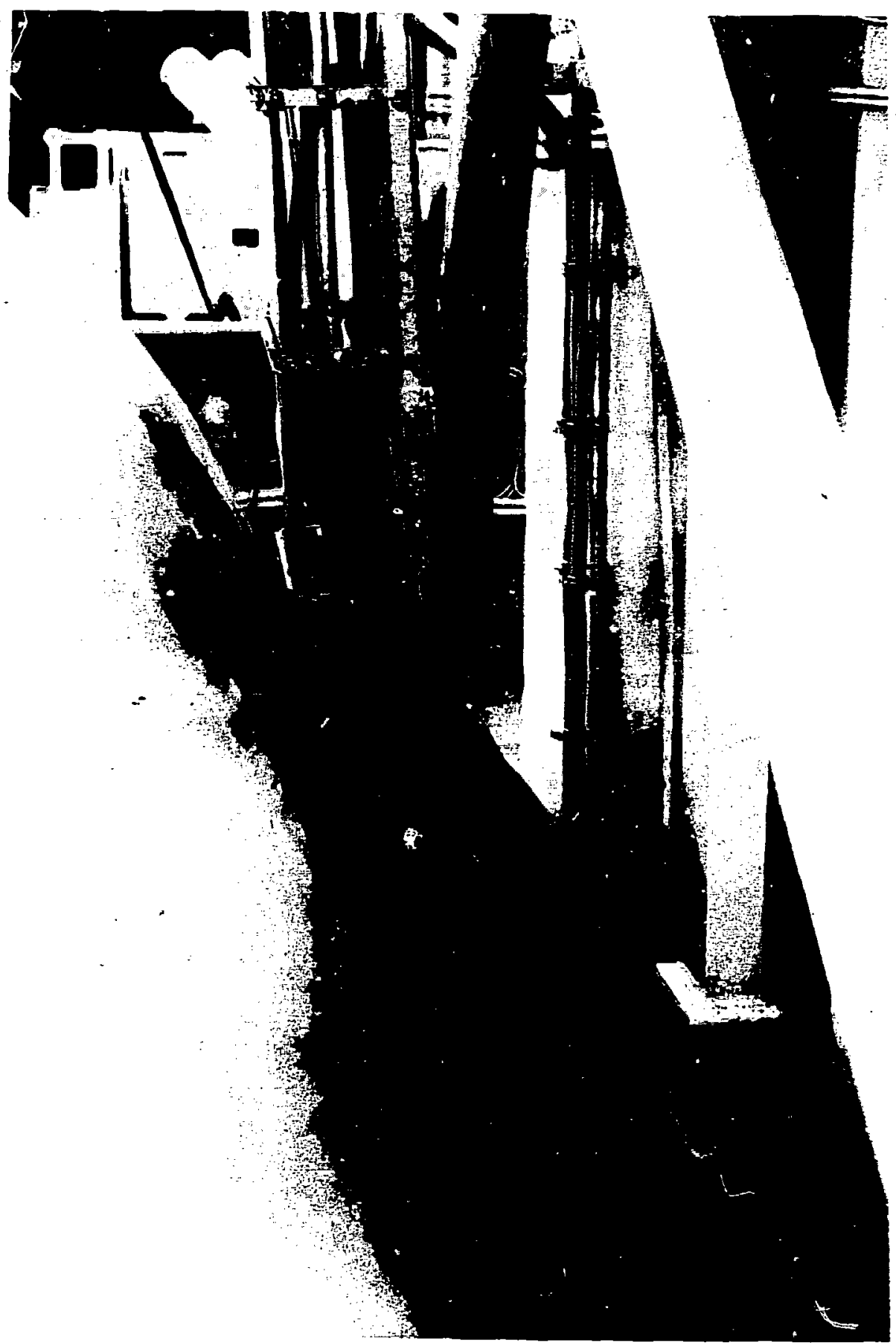

FIG. 3.8. Mast cable entry into hull. 


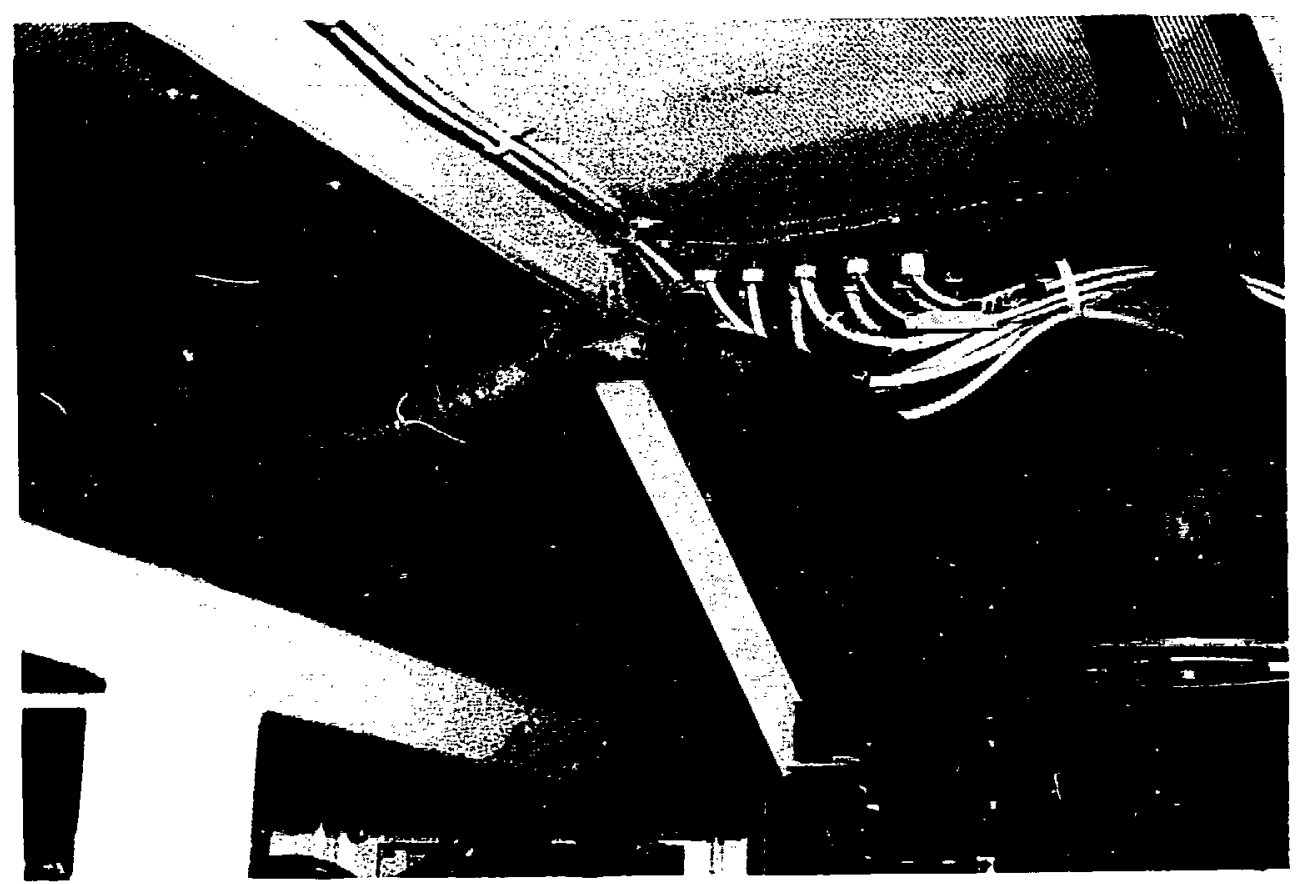

FIG. 2.9. Exit of cables below deck.

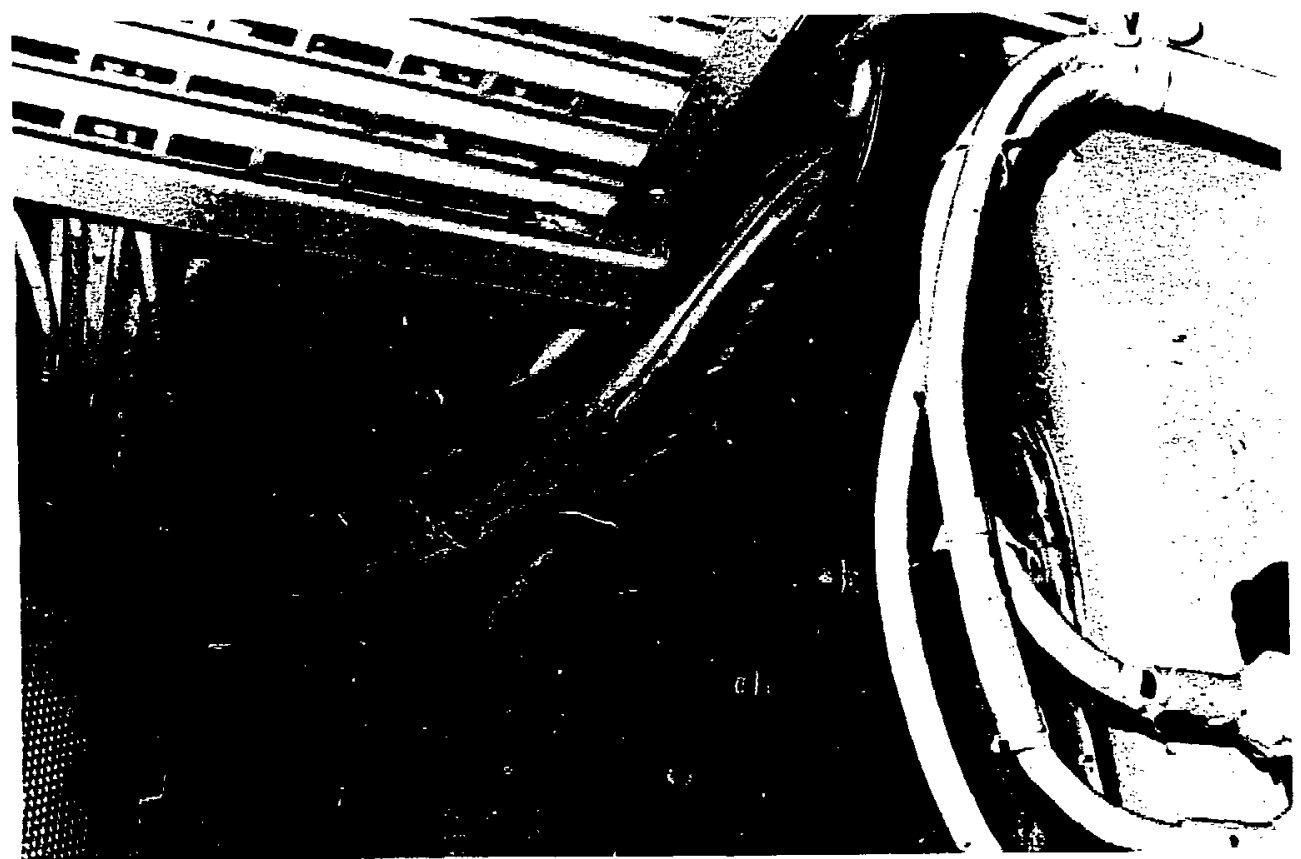

FIG. 2.10. Exit of cables below deck. 


\subsection{RREDICTIONS USING THE LIL DATA MODULES}

Table 1.1 summarizes the peak temporal open-circuit voltages, the current through a 50-ohn load, and the short-circuit currents induced at the external load terminals of the seven antennas aboard the Huron. Excitation is assumed to be from the vertically polarized mode of the EMPRess simulator. The vertical electric-field component excites the vertical antennas; the horizontal component excites the horizontal antenna on the mast.

The computations take into account (1) the effect of the wire fatness parameter ( $\mathrm{s}^{\text {inc }}$ ) of the EMPREss simulator (which is a different parameter than that exciting a reference antenna in the modules), (2) the proper geometry ratio of equivalent whip-on-boxes, (3) the angle of $\mathrm{E}^{\text {inc }}$ with respect to the antenna wires, and (4) proper scaling from a canonical monopole above a ground plane to a dipole in free space.

We used the proper scaling between a "singlet" wire (an unshielded coax center conductor representing the HF receive fans, flat tops, and the starboard AN/WLR-IC antennas) and a "doublet" wire (representing a dipole antenna at the same coax), but neglected antenna mutual interaction and clutter factors. The following paragraphs describe in detail the use of each module.*

\subsection{AS-5058 TOP MAST ANTENNAS}

Each tognast is an independent leg of a dipole which is connected to a length of $50-\mathrm{ohm}$ cable $\left(\mathrm{L} \leq 2^{\prime}=0.5 \mathrm{~m}\right)$. We use the module data to estimate $V_{L}^{\prime}=2 V_{L}$ and $I_{L}^{\prime}=I_{L}$ of load $R_{L}^{\prime}=2 R_{L}$ across a coax-fed dipole, each leg of which is of length $\mathrm{L}$. Since the modular response is for an exponential EMP wave, we correct the $V_{L}^{\prime}$ and $I_{L}^{\prime}$ responses by the factor

$$
r=\int_{0}^{T} E_{11}^{i n c}(t) d t / \int_{0}^{T} E_{11}^{E N P}(t) d t,
$$

where $T$ is either the time the first reflection at the end arrives at the load point of the dipole or the time $E^{i n e}$ first crosses zero, whichever is

\footnotetext{
tL = antenna length; $R_{L}=$ load, $\Omega=$ fatness parameter, $V_{L}=$ peak load voltage and $I_{L}=$ peak load current.
} 
shorter. $T^{\prime}$ is the first-reflection time for the EMP wave. Figure 2-7 (Ref. 1) shows peak voltage contours in the $R_{L}-L$ plane of a dipole excited by EMP in free space, and Fig. 2-6 (Ref. 1) shows the peak current contours in that plane.

\subsection{HF TRANSMIT WHIP ANTENNAS}

The response of the HF transmit whips was first estimated from the response of a comparable whip-on-box to EMP. The whip-on-box paraneter, box length/antenna length $=\mathrm{D} / \mathrm{L}$, had a value of 4.4 , where $\mathrm{L}$ equals $10.7 \mathrm{~m}$. Figure 4-35 (Ref. 1 ) shows that the $v_{L}$ for $D / L=0.85$ and $R_{L}=\infty ; 50$ ohms were estimated, as were $I_{L}$ for $R_{L}=50$ and 0 , as shown in Fig. 4-33 (Ref.1). These quantities were corrected for $\mathrm{D} / \mathrm{L}=4$ by noting the factor of change in $\mathrm{V}_{\mathrm{L}}$ and $\mathrm{I}_{\mathrm{L}}$ in going from $\mathrm{D} / \mathrm{L}=0.85$ to $\mathrm{D} / \mathrm{L}=4$, as shown in Figs. 4-24 and 4-22 (Ref. 1), where $R_{L}=300$. These quantities were then corrected by the r-factor, when $T=T^{\prime} \equiv$ time of first reflection or $35 \mathrm{~ns}$.

A second estimate of responses was made from the straight wire module. The $V_{L}^{\prime}$ and $I_{L}^{\prime}$ responses of a dipole in free space of length $L^{\prime}=2 L$ and $R_{L}^{\prime}=2 R_{L}$ were read from Figs. 2-6 and 2-7 (Ref. 1 ). The scaling rules (see page 2-4 of Ref. 1) from the dipole to monopole above the ground plane give the responses for $V_{L}=V_{L}^{\prime}$ and $I_{L}=2 I_{L}^{\prime}$. These quantities were corrected by the $\mathrm{r}$-factor and turned out to be very close to the whip-on-box responses as estimated in Tables 1.1 and 1.2 and Fig. 1.1.

\subsection{HE RECEIVE FAN (FAN "STNGLET") ANTENNAS}

Wires of approximately $18.2 \mathrm{ft}(5.5 \mathrm{~m})$ from each HF receive fan were connected to the load at one end and placed parallel to the side of the ship at a distance of about $2 \mathrm{ft} \mathrm{E}_{\text {net }}^{\text {inc }}(\omega)$, exciting the singlet, was taken as

$$
E^{i n c}(\omega) 1+j k d-(1-j k d)=j \omega(2 d / c) E^{i n c}(\omega) \text {, }
$$

Where $\mathrm{E}^{\text {inc }}$ is normally incident to the wires and reflects off the ship. Thus, $E_{\text {net }}^{i n c}(t) \cong(2 d / c) E^{i n c}(t)$, with the ship's side acting like a differentiator of the incident field. If $V_{L}$ and $I_{L}$ are the load voltages 
and current on the ship's fan, they are related to $V_{L}^{\prime}$ and $I_{L}^{\prime}$ of that fan in free space excited by $e^{i n c}(t)$ as $V_{L}=(2 d / c) \dot{V}_{L}^{\prime}$. This is also true for $I_{L} \simeq$ $\dot{I}_{L}$.

The free-space fan singlet was related to the fan doublet of the modules (see Sec. 10.5 .5 of Ref. 1) to estimate the response of the former. If the singlet has arms of length $L$ connected to a load $z_{L}^{\prime}$ with load current and voltage $I_{L}^{\prime}$ and $v_{L}^{\prime}$ and the doublet has arms of length $L$ extending in both directions from the coax of $\operatorname{load} z_{L^{\prime \prime}}^{\prime \prime}=2 Z_{L}^{\prime}$ and the response is $I_{L^{\prime}}^{\prime \prime}$ and $V_{L^{\prime}}$, then $I_{L}^{\prime}=I_{L}^{\prime \prime}$ and $V_{L}^{\prime}=V_{L}^{\prime \prime} / 2$.

The fan doublet has arms of length $L^{\prime \prime}=9.15 \mathrm{~m}$ which contrasts with the Fan where singlet $\mathrm{L}^{n}=5.5 \mathrm{~m}$. This difference was ignored, and the peak current $I_{L} "$ on the doublet through its load $R_{L}^{\prime}=600$ ohm was corrected by the factor

$$
r=\int_{0}^{T} E_{\text {ship }}^{\text {inc }} d t / \int_{0}^{T^{\prime \prime}} E_{\text {Ean doublet }}^{\text {inc }} d t
$$

where $T=18 \mathrm{~ns}$ in the first-reflection time of the ship's EMPREss incident field, and $T^{\prime \prime}$ is the zero-crossing time of the fan-doublet field.

The peak current $I_{L}^{\prime}$ of the fan singlet in free space and load $R_{L}=$ $300 \mathrm{ohm}$ was estimated as $I_{L}^{\prime}=I_{L}^{\prime \prime}$. The maximum rate of change was estimated as $\left(\dot{I}_{L}^{\prime}\right)_{P} \simeq I_{L}^{\prime} / T_{\max }$, where $T_{\max }$ is the time of $I_{L}^{\prime}$ peak. The peak current $I_{L}$ for the real ship's fan was determined to be $(2 d / c)\left(I_{L}^{\prime}\right)^{\prime}$ '

The final estimates were for a load $\left(R_{L}=300\right)$ on the ship's thr receive fan-singlet, peak current $I_{L}=1.0 \mathrm{~A}$ and $V_{L}$ peak $=300 \mathrm{~V}$. These quantities lent credibility to the straight-wire estimate of the open-circuit voltage, the 50-ohm load voltage and the short-circuit load current.

The ship's fan was represented by a straight wire of average length $\mathrm{L}_{\mathrm{eq}}=$ $5.8 \mathrm{~m}$, so $\mathrm{L}_{\mathrm{eq}}^{\prime}=11.6 \mathrm{~m}$ for a free-space center-loaded equivalent straight wire. Figure 2-42 (Ref. 1) shows that for $L_{e q}^{\prime}=10 \mathrm{~m}$, a peak load voltage $V_{L}^{\prime \prime}=2.8 \times$ $10^{5}$ at $t^{\prime \prime} c / L_{e q}^{\prime}=0.7$ or $t^{\prime \prime}=23 \mathrm{~ns}$, when $R_{L}^{\prime \prime}=1 \mathrm{M}$. Thus $\dot{V}_{L}^{\prime \prime}$ peak $\simeq 1.22 \times$ $10^{13}$. From the above relations $\dot{V}_{L}^{\prime}$ peak on the fan-singlet wire of load $R_{L}=$ $500 \mathrm{~K}$ would be half this or $0.61 \times 10^{13} \mathrm{v} / \mathrm{s}$. Therefore, the peik opencircuit voltage on the ship's fan-singlet wire is estimated as $V_{L}$, where the 500-ohm load $\simeq(2 \mathrm{~d} / \mathrm{c}) \dot{\mathrm{V}}_{\mathrm{L}}^{\prime}=41 \mathrm{kV}$. This is then corrected by the $r$-factor of 3.11, and our final result for Table 1.2 is $V_{L}$ peak $=910 \mathrm{~V}$.

Similar computations yield $I_{L}$ peak for $R_{L}=$ ohm and 0 ohm in Table 1.2 . 


\subsection{AN/SRC-23 FLAT TOP ANTENNAS}

Each Elat top antenna is a fan arrangement of wires cunning from a terminal point on the deck to various points on the mast where they connect to a RG 218 cable. The effective load point is at an intermediate point on the mast.

A straight-wire estimate of the response was obtained by computing effective fatness parameter $\Omega=19.8$. The free-space straight-wire doublet response and the "sing?et" Elat top free-space response were estimated as above for the HF receive fan. For example, the peak open-circuit voltage $V_{L}^{\prime}$ for $R_{L}^{\prime}=1 M$ of the doublet antenna with total length $\mathrm{L}^{\prime}$ eq $=40 \mathrm{~m}$ was obtained from Fig. 2-7 (Ref. 1 ) of the modules as $v_{L}^{\prime}=10^{6} \mathrm{~V}$. This is corrected for $\Omega$ by Fig. 2-17 (Ref. 1 ) and is further corrected by the cosine nf the angle between $E^{i n c}$ and the plane of the wires. After correcting by ne $r$-factor, we obtain $v_{L}^{\prime}=24 \mathrm{kV}$. Then the singlet straight-wire peak voltage across $R_{L}=$ $500 \mathrm{~K}$ is $V_{L_{L}}=1 / 2 V_{L}^{\prime}=12 \mathrm{kV}$. This is the module estimate of open-circuit load voltage on the flat top antennas.

Corresponding computations are made for $I_{L}$. All these numbers are compatible with a $\mathrm{Ean}$-doublet estimate of $I_{L}=23 \mathrm{~A}$ at $R_{L}=300 \mathrm{ohm}$.

\subsection{AS-5048 (PORT) AND AS-5058 (STARB)/AN/WLR/1C ANTENNAS}

These are short $(1.07$ and $1.22 \mathrm{~m})$ monopole-like antennas on the mast. We ignore the considerable effect of the tower and estimate the antenna responses for Table 1.1 just as we did the AS-5058 top mast antennas.

\subsection{AN/SRN-12 OMEGA ANTENNA}

First we estimated the response by an equivalent center whip-on-box. Fatness parameter $\Omega=11.5 \simeq 10$. The box-length-to-antenna-length ratio $\mathrm{D} / \mathrm{L}$ was estimated as $156 \mathrm{ft} / 10 \mathrm{ft}=15.6$. From Fig. 4-23 (Ref. l) for $D / L=10$, $L=3 \mathrm{~m}$, and $R_{L}=300$, then $V_{I}=1.2 \times 10^{5}$. From Fig. 4-21 (Ref. 1 ), $I_{L}=400$ A. From Fig. 4-23 (Ref. 1), $V_{L}$ decreases by a factor 0.3 in changing from $D / L=$ 0.85 to $10 ; I_{L}$ decreases too. From Fig. 4-34 (Ref. 1 ), for $D / L=0.85$, and $L=3 \mathrm{~m}$, then $V_{L}=8 \times 10^{5}$ for $R_{L}=1 \mathrm{M}$, and $V_{L}=1 \times 10^{5}$ for $R_{L}=50$. From Fig. 4-32 (Ref. 1 ): for $D / L=0.85$, and $L=3 \mathrm{~m}$, then $I L=2 \times 10^{3}$ for $R L=$ 
50 , and $I_{L}=2.5 \times 10^{3}$ for $R_{L}=1$. Hence, for $D / L=0.85$, we estimate $V_{L}$ open-circuit $=8 \times 10^{5}, I_{L}$ for a $50 \mathrm{ohm}$ load $=2 \times 10^{3} \mathrm{~A}$, and $I_{L}$ short-circuit $=2.5 \times 10^{3} \mathrm{~A}$.

The correction factor changes when $D / L$ goes from 0.85 to 10 , and is the same correction factor $(0.3)$ used above for $R_{L}$ - 300. A further correction is by factor $r$, where $T=T^{\prime}=10 \mathrm{~ns}$. The final whip-on-box response estimates are as quoted in Table 1.2 .

For a straight-wire check of these figures, we approximate the whip antenna on the ship by a monopole above a ground plane. We obtain from the module the free-space EMP response of a dipole of twice the length of the whip, where $V_{L^{\prime}}^{\prime}$, for $R_{L}^{\prime}=1 \mathrm{M} 1 \mathrm{~s} 1.8 \times 10^{5} \mathrm{~V}, I_{L}$ for $R_{L}^{\prime}=100 \mathrm{ohm}$ is $300 \mathrm{~A}$, and $I_{L^{\prime}}$ for $R_{L}=1$ is $500 \mathrm{~A}$. By the scaling rules from dipole to monopole, we obtain for the latter $V_{L}$, for $R_{L}=500 \mathrm{~K}$ is $1.8 \times 10^{5}, I_{L}$ for $R_{L}=50$ is $600 \mathrm{~A}$, and $I_{L}$ for $R_{L}=0.5$ is $1000 \mathrm{~A}$.

Since these last three responses are so close to the corresponding whipon-box numbers, we use the whip-on-box entries in Table 4.1 (Ref. 1) before making the r-correction of $2.4 \times 10^{5}, 600$, and 750 , respectively.

\subsection{AT-924B/HORIZONTAL ANTENNA}

According to the description of the EMPRESs facility, the verticalpolarized mode has a horizontal $E_{p}$-component of about 108 of the vertical, which directly excites the antenna. Because we cannot estimate the response of the total, vertical plus horizontal driving field with the modular data measured on the Transient Electromagnetics Range, we estimate $V_{L}$ and $I_{L}$ of a single wire connected to a coax from $V_{L}^{\prime}$ and $I_{L}^{\prime}$ of the equivalent dipole in free space, just as we did for the As-5058 top mast antenna. In the correction factor $r, E^{i n c}=E_{p}^{i n c}$ and $T=T^{\prime}=$ time to first reflection, or about $15 \mathrm{~ns}$ on the dipole.

\subsection{MAST (Base of one leg)}

The mast is simply treated as four vertical monopoles shorted to a ground plane (deck), each of length $L=50 \mathrm{ft}$. Neglecting interaction, the module, (Fig. 2-6 of Ref. 1) gives, for a dipole of length $\mathrm{L}^{\prime}=100 \mathrm{ft} \simeq 30 \mathrm{~m}$ in free space, short-circuit current $I_{L}^{\prime}=2.2 \mathrm{kA}$. So by the scaling rule from 
dipole to monopole, we obtain $I_{L}=4.4 \mathrm{kA}$ for the mast current at the base of one leg. The correction factor $\mathrm{r}$ of $\mathrm{Eq}$. (3.1), with $\mathrm{T}=\mathrm{T}^{\prime}=50 \mathrm{~ns}$, and time of first reflection is $r=94 / 2355$. Hence our estimate for mast leg current at the deck is $4.4 \times 10^{3} \mathrm{r}=176 \mathrm{~A}$ in 3 ble 1.2 . 


\subsection{LLL 1/48-SCALE MODEL OF HMCS HURON}

All measurements reported here were made on a $1 / 48$-scale model of the HWCS Huron. Figure 4.1 shows the model placed on a ground plane in front of a monocone antenna. The model, constructed at LLL, is 243.8-cm long, 31.75-cm wide, and $87-\mathrm{cm}$ high at the top of the mast above the water line. (Note that the overall length of the aft main deck was shortened by $8.9 \mathrm{~cm}$ in order to accommodate available building materials.) The hull and superstructures are covered with 1/32-in. brass sheet to form a conductive body, while the main mast is constructed with $1 / 8-$ and $1 / 16-i n$. brass welding rod.

Particular detail was given to the mast and the equipnent bays, since they are the areas of primary interest in the internal coupling measurement. A modular approach was employed with the equipment rooms, and metal room dividers were installed to separate the various areas internal to the ship. Each of the three decks were made removable to facilitate the installation of cables and sensors used in the tests.

Figure 4.2 shows an outline drawing of the Huron model with the locations of the onboard antennas denoted. We used SMA series bulkhead feedthrough connectors to mount the ship antennas to the ship's hull. These connectors provide a very convenient way of mounting the antennas to the ship, forming a 50-ohm antenna feed region between the grounded outer shield and the center conductor. Fifty-ohm coaxial cables and connectors were used for all the measurements.

The four individual wires of each of the HF/receive fans and the flat top antennas were electrically joined at the feed ends and individually insulated at their far ends from the ship's hull, thus forming "singlets," as discussed in Sec. 3. We also used SMA series elbow connectors to form a feed for the two AT924B horizontal singlets mounted on the main mast. The coax feed for the AT924B antennas as well as the flat top antennas were fed down one leg of the tower, through a hole in the ship's hull to the monitoring instrumentation located beneath the ground plane. More details on how the model measurements were actually made are discussed in Refs. 2 and 3 as well as in Sec. 5 of this report.

Details of the cable runs internal to the hull of the Huron are less well-defined than those on the externally mounted antennas. Basically, we modeled the coaxial cables and control wires running from the equipment bays 
and up the mast to their respective antennas. We were only interested in bulk cable currents--the currents flowing in the outer shields of the coaxial cables. Specific details of the wires and cables were simply not available, nor was information as to there or if the outer shields were even grounded. In general, we assumed that the cables would be grounded to the equipment racks or the hull interlor; at the opposite ends of the cables wires were either grounded to the mast or left floating.

In total, we measured the currents in six equipment compartments. A description of the cables leading to each of the six compartments follows.

\subsection{EQUIPMENT COMPARTMENT CABLE DESCRI PTIONS}

\subsubsection{Electronic Warfare Equipment Room (EWER)}

The EWER is located on the 02 deck next to the mast. A 19-wire bundle $r$ uns halfway up the fiberglass mast to the As-5058 top mast assembly, down the mast to the rear of the EWER room, through an opening into the EFER where the bundle is grounded. A second cable is routed from a connection on the mast at level J (see Fig. 1.1), down the mast, and Into the ENER, where it is grounded to the ceiling of the EWER. This is the cable monitored.

\subsubsection{Transmitter Room No. $2(\mathrm{mX}-2)$}

The cable leading to the No. 2 transmitter room is connected at one end to the base of the platform at the top of the mast. This cable is also fed down the mast with the bundle leading to the EwER. The cable first enters the EWER and then passes on through at ceiling level to the TX-2 room, where it is terminated on the room celling. We measure this cable in the TX-2 room.

\subsubsection{Electronic Warfare Control Room (EWCR)}

The EACR and CCR are both on the 01 deck. A 20-wire bundle from the base of the TACAl enters the ERCR through a portgide hole in the BWCR ceiling where 10 of the wires are terminated in the EmCR bay. The cable monitored is an open circuited wire from the top of the fiberglass mast, fed down the mast and into the EMCR, where $1 t$ is grounded. 


\subsubsection{Combat Control Room (CCR)}

The other 10 wires of the 20-wire bundle leading to the ExCR are fed through the EWCR and through the wall leading to the CCR. The 1.0-wire bundle is terminated in the CCR. The cable monitored is grounded to a horizontal strut on the mast at level $J$, fed down the mast, through the EncR and into the CCR where it is also grounded.

\subsubsection{TACAN ROOM}

The TACAN and TX-1 rooms are located on the main deck. Crbles feeding these rooms enter from above through the 02 deck and pass vertically through the EMCR compartment. The cable monitored in the TaCAN room is grounded to the base of the TACAN antenna on the main mast, and terminates in the TACAN room after passing through the 02 and 01 decks.

\subsubsection{Transmitter Roon (TX-1)}

Finally, the cables leading into the TX-1 transmitter room are connected to the ends of the flat-top antennas, one on the port side and one on the starboard side. We monitored the portside cable.

Figure 4.3 shows the general routings of the cables down the mast and into the equigment bays. Without an on-site inspection it is impossible to determine the exact routings of the cables leading to the various equipment rooms. It is also unknown if and where the cables are grounded. All of these factors can have a significant effect on the currents measured and the scaled-up results obtained. 


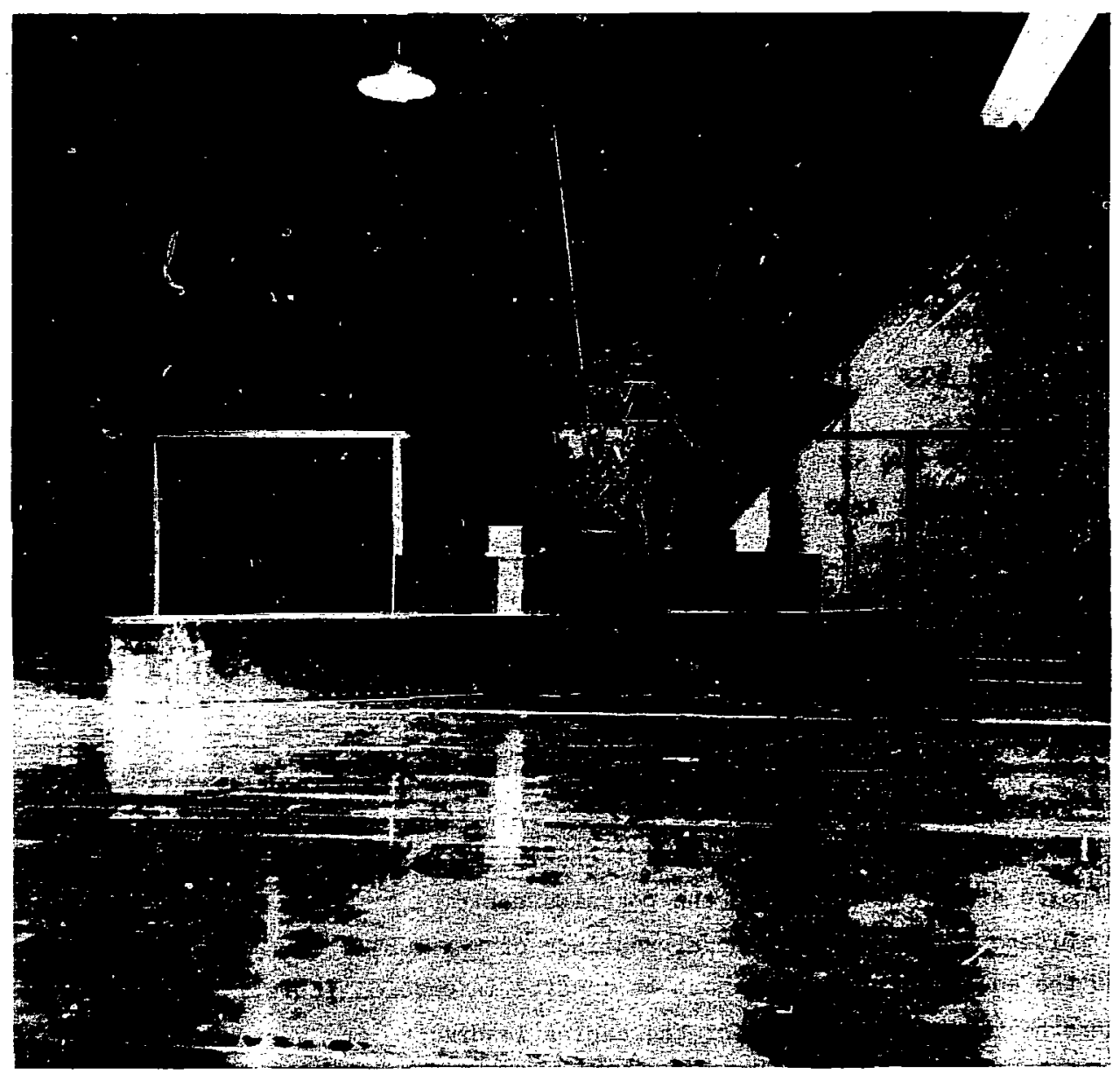

FIG. 4.1. 1/48-scale model of Hucs Huron on ground plane in front of monocone simulator antenna. 


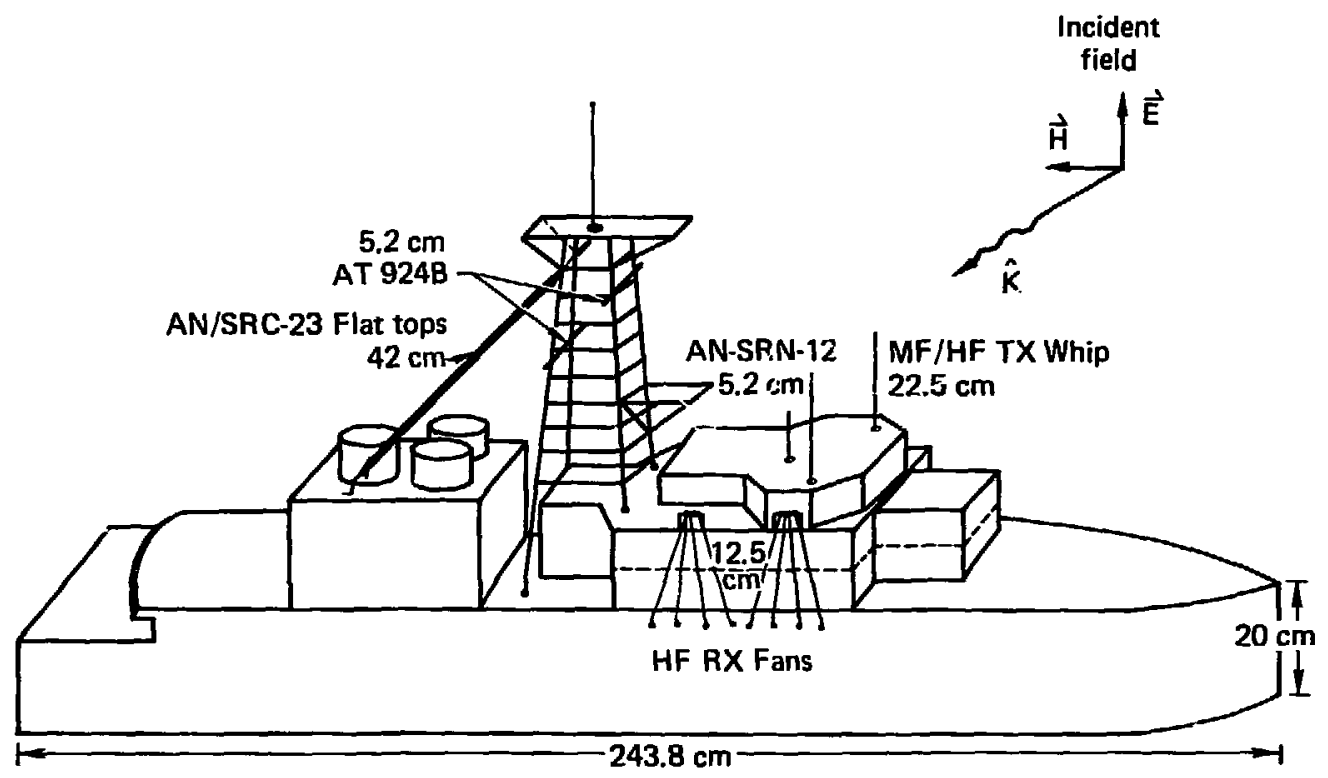

FIG. 4.2. Antenna locations on 1/48-scale model of HMcs Huron.

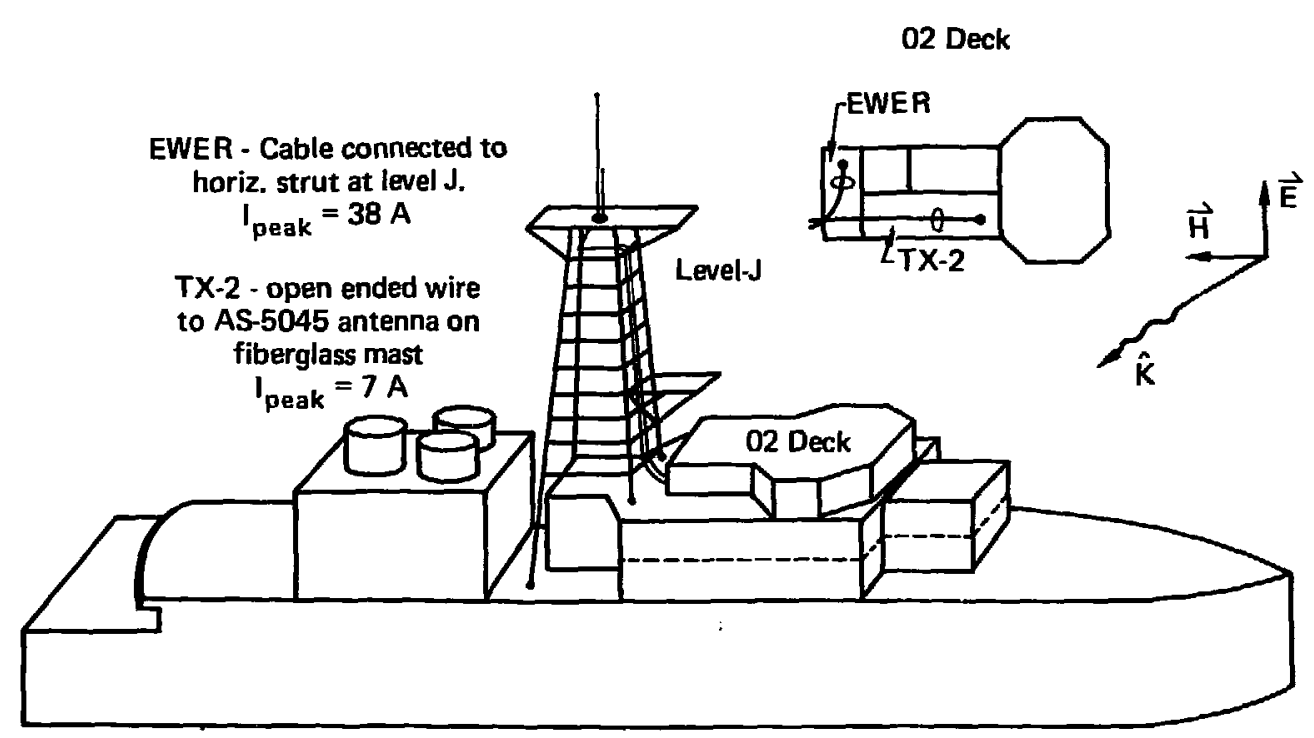

FIG. 4.3a. Cable runs from mast to equipment compartments (02 deck). 
EWCR - open ended wire to top of fiberglass mast through ceiling of 01 Deck $I_{\text {peak }}=17 \mathrm{~A}$

CCR - wire grounded to top of mast at level

J.-through 01 Deck and EWCR

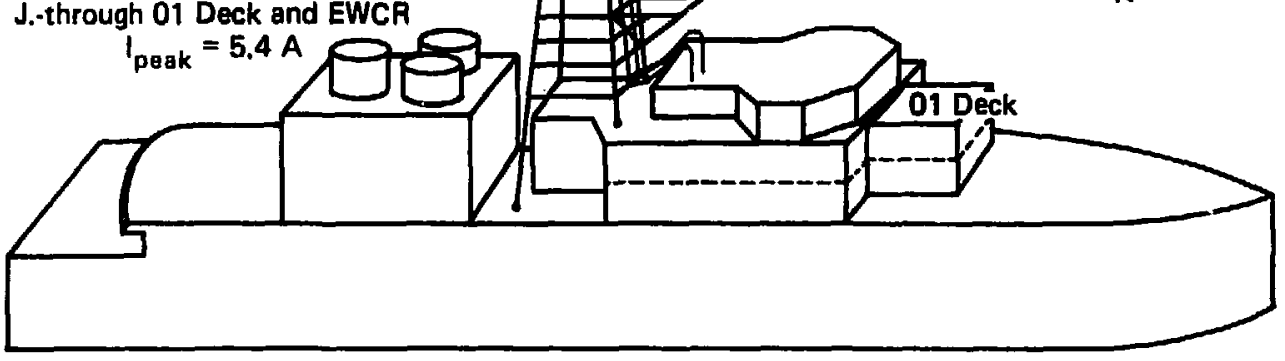

FIG. 4.3b. Cable runs from mast to equipment compartments (0l deck).

TACAN room - wire connected to base of TACAN antenna through 01 Deck. $I_{\text {peak }}=24 \mathrm{~A}$

TX-1 - wire connected to port side flat top through 01 Deck and TACAN room

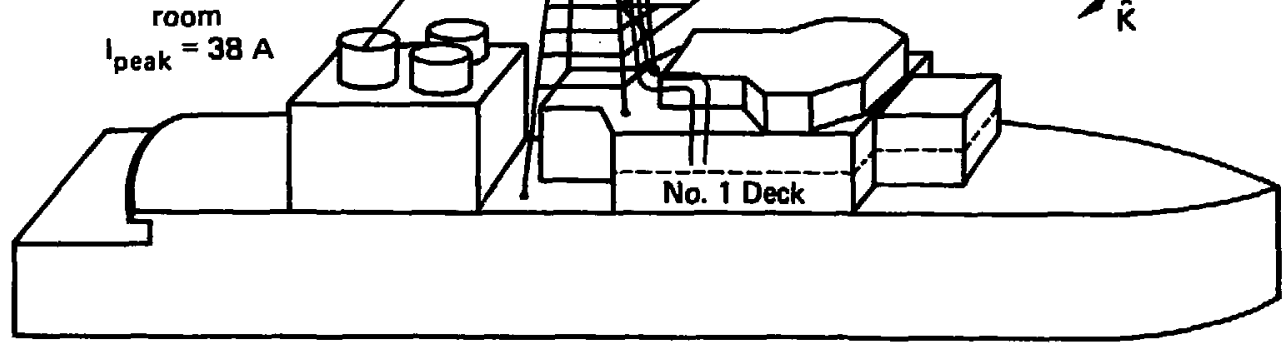

FIG. 4.3c. Cable runs from mast to equipment compartments (no. 1 deck). 


\subsection{SCALE MODEL MEASUREMENT TECHNIQUES AND MEASURED RAW DATA}

Scale model EM measuremerts used for coupling assersments may be performed in either the time or frequency domains. LLL has pursued the time domain approach, cieveloping techniques to characterize the coupling properties of systems by making a few simple tranident measurements. ${ }^{2,3}$ These techniques, while aiscussed more fully in the referenced reports, are briefly discussed in the sections below. Representative raw data are also shown to illustrate the data obtained by LLL on the Huron model.

\subsection{IMPULSE SOURCE FIELDS}

Embodied in the time domain measurement technique is the use of very wide Dandwidth incident EM fields or excitations to force the response of a system over its full spectral range. At LLL we used a very fast rise-time impulse generator to drive a wideband radiating antenna. This impulse (300-ps wide, $1500 \mathrm{~V})$ feeds the apex region of a monocone antenna, which in turn radiates a vertically polarized TEM wave from the apex of the cone. Figure 5.1 shows the measured $\mathrm{EM}$ pulse produced by the impulse generator/monocone arrangement, and its equivalent frequency domain spectrum is shown in Fig. 5.2. As can be seen from Fig. 5.1, the peaks pulse amplitude is approximately 700 volts/meter at the measurement point, while in the frequency domain, the spectrum is fairly uniform to $3.5 \mathrm{GHz}$.

\subsection{TRANSIENT SENSORS AND PROBES}

A very important aspect of transient $E M$ scale modeling is the availability of sensors and probes to faithfully measure and respond to the incident EM fields and induced signals. For the antenna coupling measurements this is simple--the antenna itself is used as the sensor.

In order to measure the transient EM fields, we utilized two small $\dot{B}$ and D sensors developed by the Air Force Weapons Laboratory. The outputs from these sensors, which are shown in Fig. 5.3, must be integrated to obtain the electric and magnetic fields. (Note that the incident vertical electric field shown in Fig. 5.1 was measured with the ACD-1 $\dot{D}$ sensor.) The bandwidth of these sensors extends to greater than $7 \mathrm{GHz}$, which is beyond the useful frequency range used in this experiment. 
All of the bul: current measurements were made with a third sensor, a Tektronix CT-1 current probe, as shown in Fig. 5.4. These probes are used to measure current flow on wires and have a bandwidth of 1-GHz.

The outrut signals from the antennas, probes, and sensors are all fed to a transient recording system that consists of a computer-controlled sampling oscilloscope. The analog data from the oscilloscope are converted to a digital format by an $A / D$ converter in the computer, and the data representing the transient waveform are output onto magnetic tape for off-line post processing.

The next two sections present representative scale model data obtained for external and internal EM coupling to the Huron, as obtained on the LIL transient EM range.

\subsection{EXTERIOR COUPLING}

When an incident electromagnetic pulse illuminates a complex structure such as the Huron model, currents are induced in the whole structure. This is also true of external energy collectors such as the antennas and towers aboard the ship. Our interests here are in the signals coupled to the ship's antennas, since it is these receptors that connect to the sensitive transmitters and receivers where burnout can occur.

We used an equivalent circuit to characterize the external shipboard antennas. Figure 5.5 shows the frequency domain antenna equivalent circuit form used, where $z_{a}(f)$ is the port impedance of the antenna, $H_{e f f}(f)$ is a quantity called the effective height of the antenna, and $E_{\text {inc }}(f)$ is the incident electric field, where all parameters are specified in the frequency domain. Two of these parameters, $\mathrm{Z}_{a}(f)$ and $\mathrm{H}_{\text {eff }}(f)$, are unknown quantities that are characteristic of the antenna which must be experimentally measured with the antenna in place. $E_{\text {inc }}(f)$ can be any incident electric field, and, in our measurements, it is the electric field produced by the monocone source antenna. $z_{L}(f)$ may be any value of load impedance connected to the terminals of the antenna.

The advantage of using an equivalent circuit of this form is the flexibility it affords. The effective height measured on the scale model scales linearly with model size, while the frequency range scales inversely with model size. The impedances, $z_{a}$ and $z_{L}$, do not scale with model size; only the Irequencies at which they are defined. 
The load impedance can be any complex value. If $\mathrm{Z}_{L}=0$, we can find the short-circuit current, and if $z_{L}=\infty$, we can compute the open-circuit voltage across the antenna terminals. Similarly, once the parameters of the equivalent circuit are determined, the response of the antenna to any incident field may be determined, e.g. an EMP waveform or the incident field produced by the EMPRESS simulator.

While the equivalent circuit is primarily a frequency domain model, the frequency domain results may be Fourier transformed to the time domain to give the time history waveform. This is the technique used here.

Specific details of the procedures used to determine the antenna input impedance, $\mathrm{z}_{a}(f)$, and the effective height, $\mathrm{H}_{\text {eff }}(f)$, are given in Ref. 2 . The technique, in general, employs a TDR (time-domain reflectometry) process to obtain $z_{a}(f)$. With $z_{a}$ known, the scale model is illuminated by a known incident field, $E_{\text {inc }}$. The response is measured at the antenna terminals across a known load impedance. With $E_{\text {inc }} \mathrm{z}_{a}$ and $\mathrm{z}_{1}$ known, the Thevenin equivalent circuit is solved to determine $\mathrm{H}_{\text {eff }}$.

To illustrate this procedure, the measurements for one of the scale model 35-ft whip antennas will be shown in detail. The first step is to make a TDR measurement to determine $\mathrm{z}_{\mathrm{a}}(\mathrm{f})$. We do the TDR measurement by making two measurements of the pulse reflected from the end of a feed line, first with the line left open at its end, and second with the subject antenna connected to the end of the feed line. The experimental configuration, and the respective measured TDR waveforms for the scale model 35-ft whip antennas are shown in Figs. 5.6a and 5.6b. Figures 5.6c and 5.6d show the respective Fourier transforms of the reflected pulses. Note that the spectrum of the open-circuit, reflected pulse is fairly flat, while the spectrum of the reflected pulse with the antenna connected as a load contains some definite structure. Once we have the two TDR transforms, a reflection coefficient, $\rho(f)$, can be found as:

$$
\rho(f)=-\frac{v_{\text {load }}(f)}{v_{\text {open }}(f)}
$$

then, with $\rho$ (f) known, the characteristic impedance can be found as:

$$
z_{a}(f)=\frac{1-\rho(f)}{1+\rho(f)} \star z_{0}
$$


where $z_{0}$ is the characteristic impedance of the antenna feed line used in the TDR measurement. The real and imaginary part of $z_{a}(f)$ are shown in Figs. 5.6e and $5.6 \mathrm{f}$.

The second half of the experiment determines the antenna's effective height. This involves placing the ship model on the transient range ground plane and illuminating it with a known incldent field like that shown in Figs. 5.1 and 5.2. The transient response of the antenna under test, measured at the end of the antenna feedline, is shown in Eig. $5.6 \mathrm{~g}$ in the time domain, and then in Fig. 5.6h transformed to the frequency domain.

The effective height, $B_{\text {eff }}(f)$, may then be found from the equivalent clrcult. Figure 5.61 shows the experimentally determined plot for the scale model 35-ft antenna effective height. With these parameters, the equivalent circuit model may be scaled up to a full-sized structure, and the response to any incident waveform may be found.

Similar measurements were made for the other antennas on the ship model. The input impedance and effective heights for these other antennas are shown in Figs. 5.7 through 5.13 .

Besides the ship's antennas, the main mast also responds to the incldent wave. Sizable currents flow on the mast, and these currents influence, in turn, the responses of the antenna and other receptors on the ship. Figure 5.14 is a close-up of the mast section on the scale model of the Huron. We made several current measurements on the mast by utilizing the CT-1 current probe shown previously in Fig. 5.4.

Figure 5.15 shows the points at which the mast currents were measured. Figures 5.16 through 5.19 show the raw time-domain currents measured on the scale model and the current transfer function relating the current to the incident $E$ field. (Note: These plots are scaled up by the $\times 48$ scale factor.) Note that these currents are for one leg only of the mast. The forward, port side member was chosen for this test.

\subsection{INTERIOR COUPLING MEASUREMENTS - RAW DATA}

As discussed earlier, the measurements of the internal Ex coupling consisted of a measure of the bulk cable currents in the interior bays of the ship model. The cables measured were primarily those running from the various antennas on the main mast, down the mast, and into the equipment compartments 
of the ship. Here the bulk-cable current is shield current. It should be noted that with the 1 imited information on cable runs, groundings, and connections, it was not possible to accurately model the actual cable runs on the ship. The measurements obtained are thus only "ball park" coupling values that indicate the levels of signals which may be obtained.

All the internal bulk current measurements were made with the CT-l current probes. These probes were located at the ends of the cable runs in each of the six interior rooms of the Huron model. The raw data shown in Figs. 5.20 through 5.25 were scaled-up by 48 in amplitude, which corresponds to the model scale factor, and similarly were scaled-up by 48 in the time scale. The measured time-domain transient currents are shown first in each figure set. These currents are the response due to the impulse source field as shown in Fig. 5-1. The second of each figure set shows the transfer function in the Erequency domain that relates the bulk cable curcents to the incident field over a wide frequency range. Section 6 shows the responses for the EMPRESS incident field. 


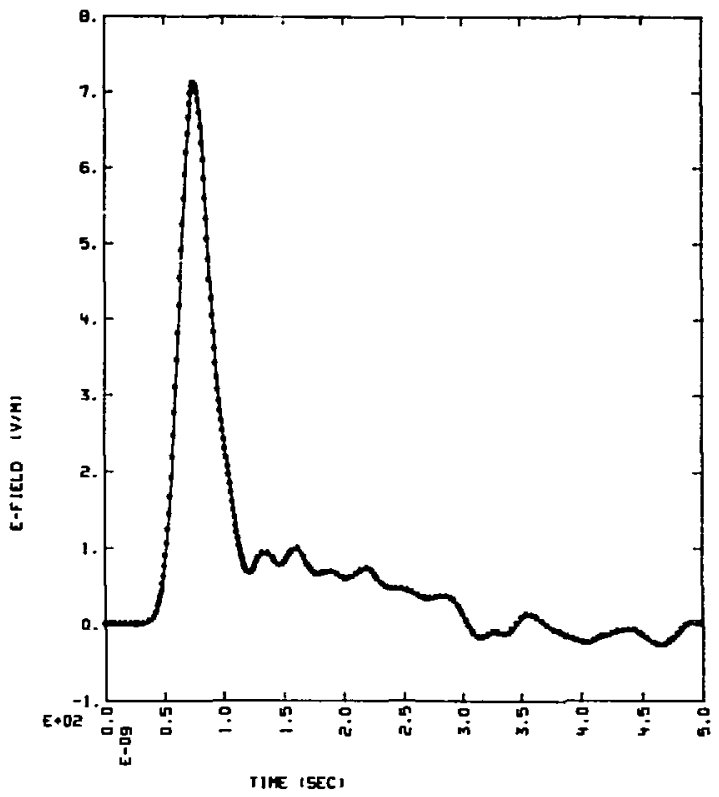

FIG. 5.1. Measured vertical incident field at location of Huron measurements.

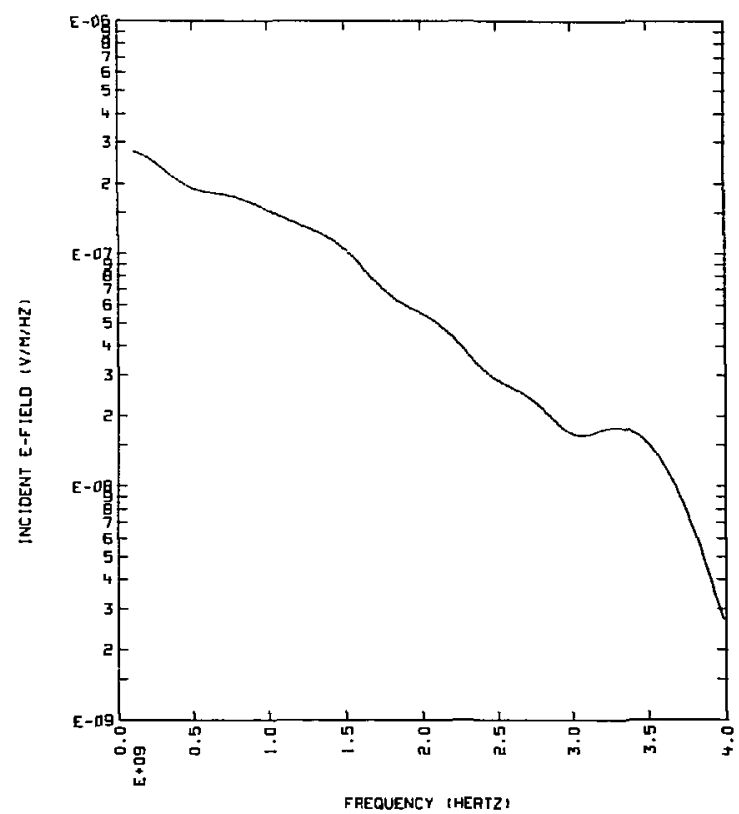

FIG. 5.2. Frequency spectrum of incident electric field. 


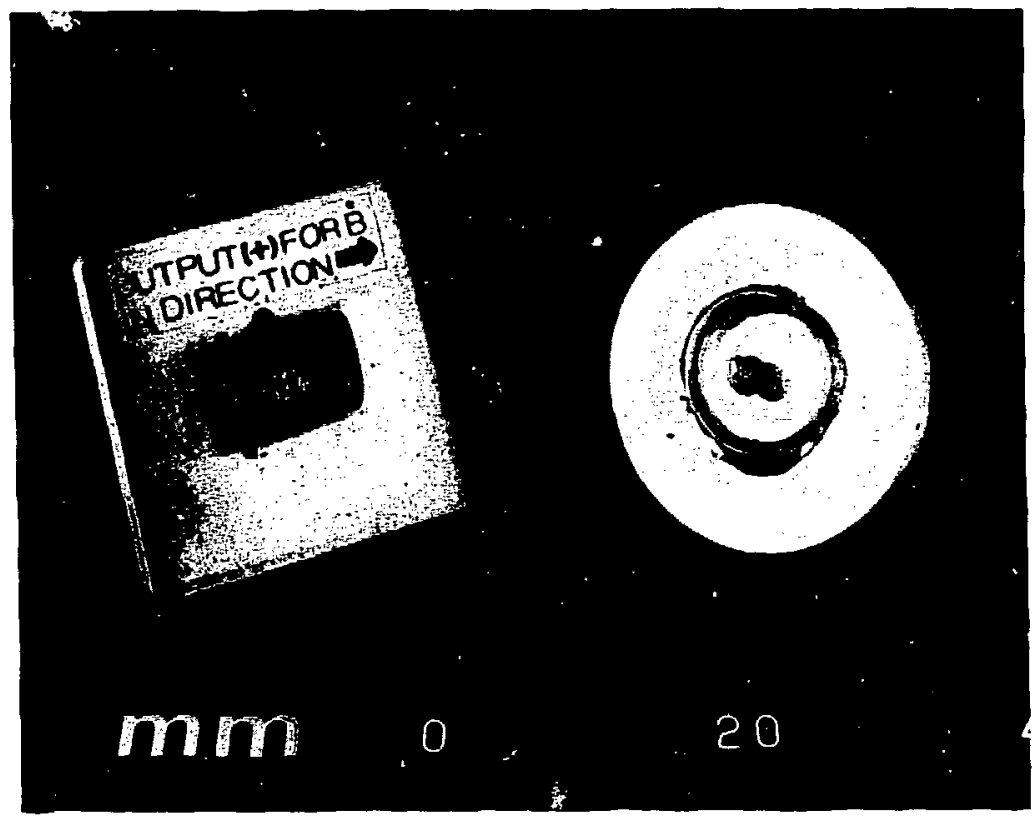

FIG. 5.3. Subminiature $\dot{B}$ and $\dot{D}$ probes. 


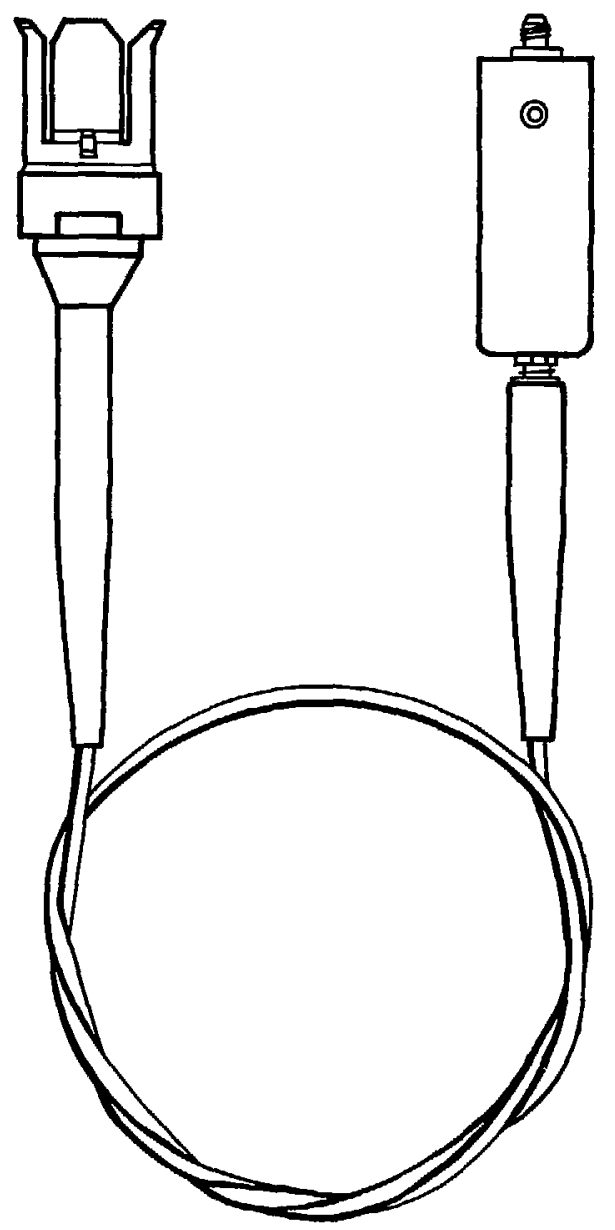

FIG. 5.4. Tektronix CT-1 current probe.

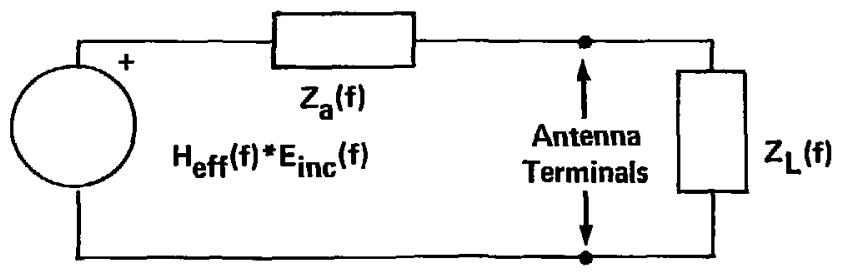

FIG. 5.5. Frequency-domain antenna equivalent circuit.

$5-8$ 


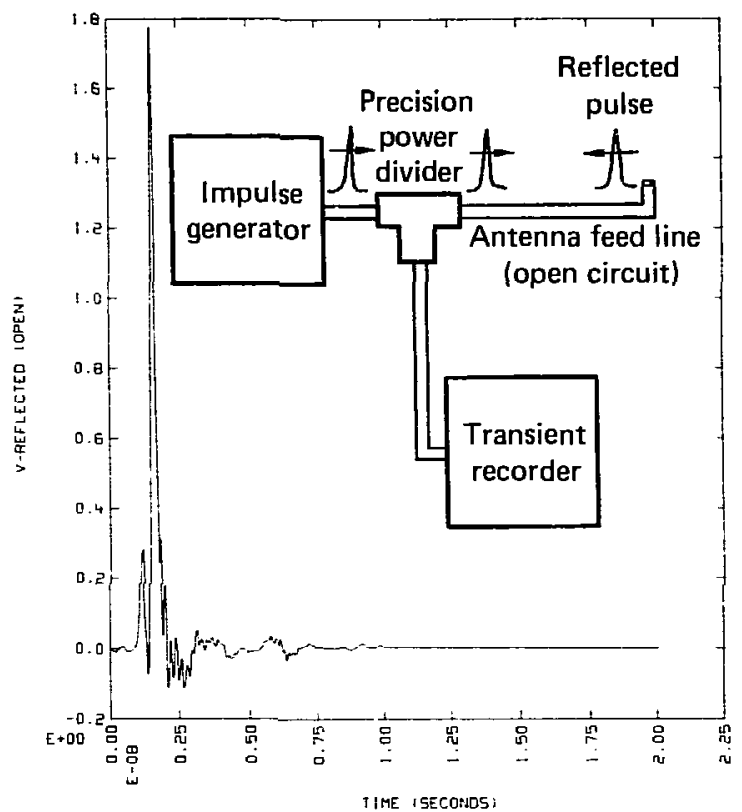

FIG. 5.6a. Reflected voltage with antenna feedline open circuited.

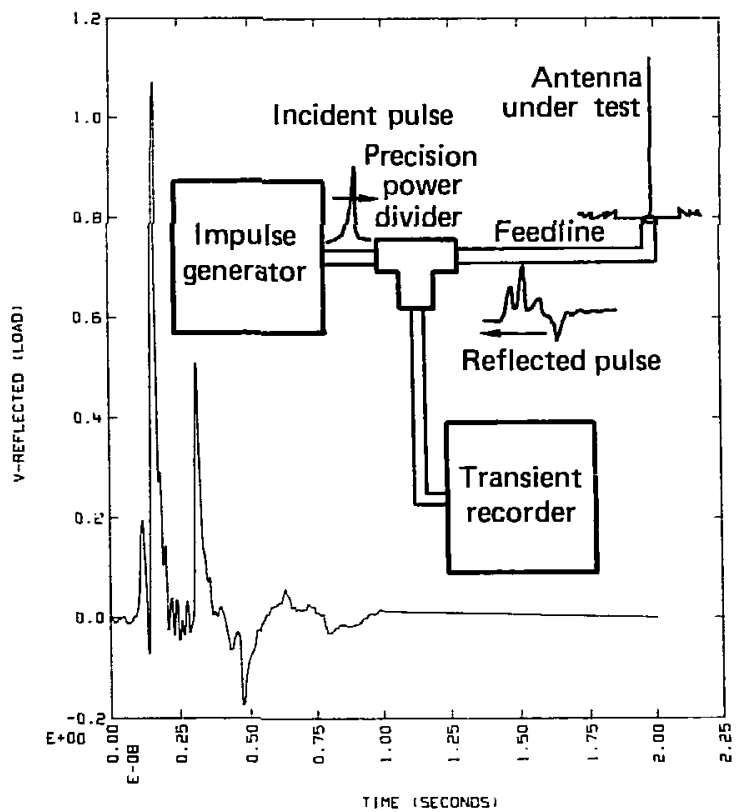

FIG. 5.6b. Reflected voltage with antenna connected to end of feedline. 


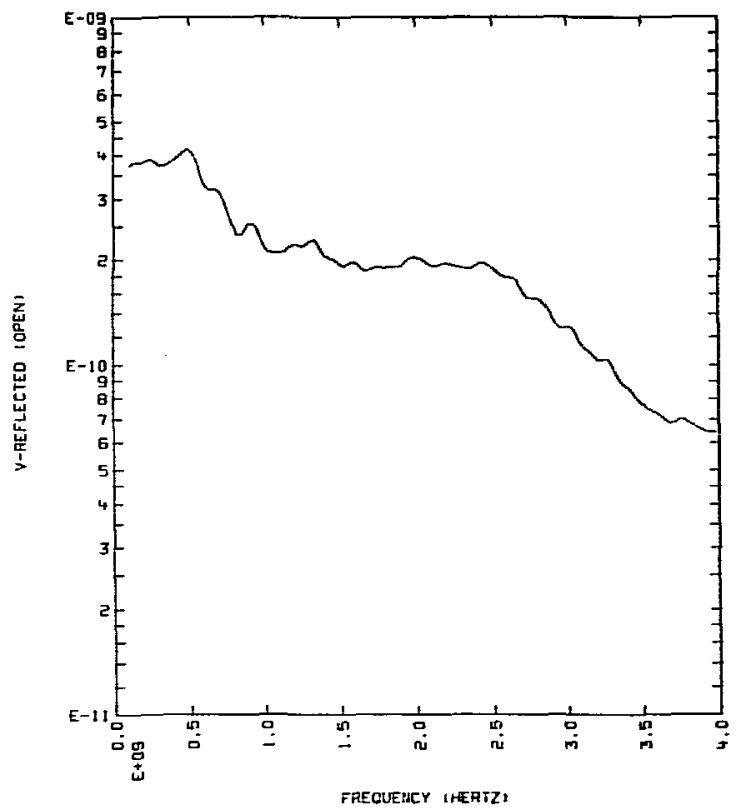

FIG. 5.6c. Fourier transform of open circuit reflected voltage (Fig. 5.6a).

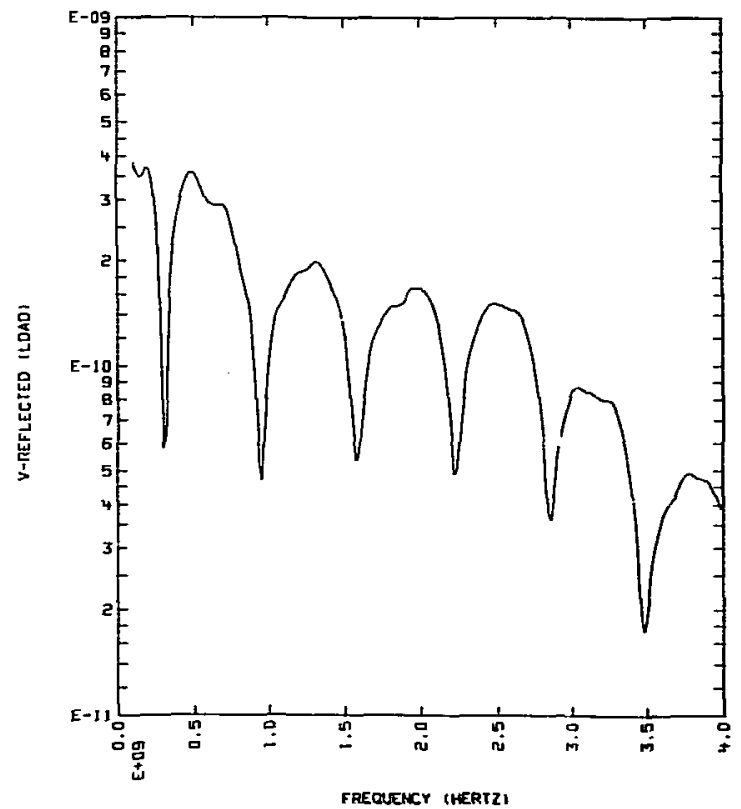

FIG. 5.6d. Fourier transform of reflected pulse with antenna connected to feedline (Fig. 5.6b). 


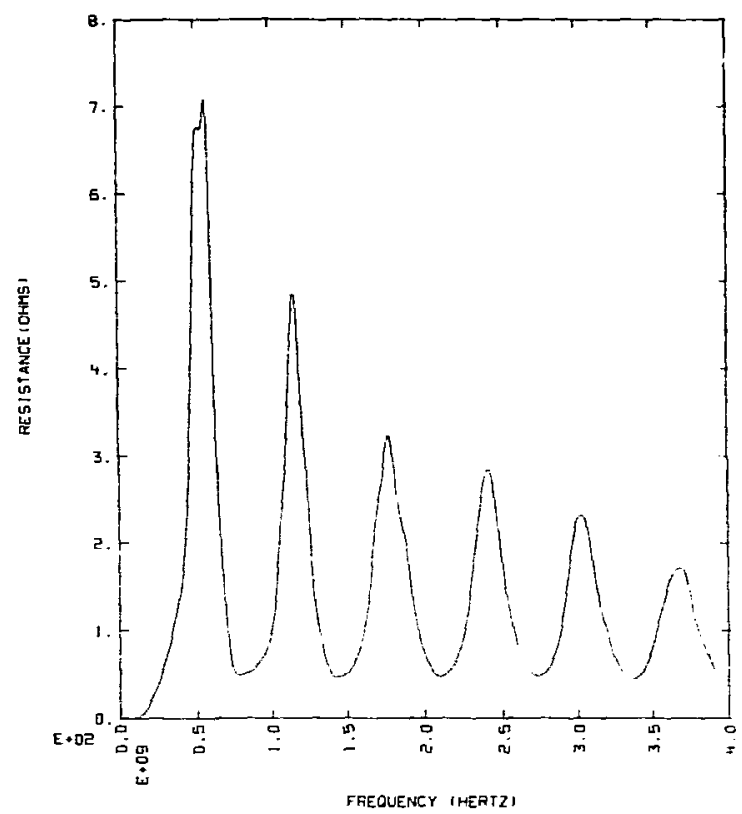

FIG. 5.6e. Real part of antenna impedance, $\operatorname{Re}\left(z_{a}\right)$, as a function of frequency for scale-model $35-\mathrm{ft}$ whip antenna.

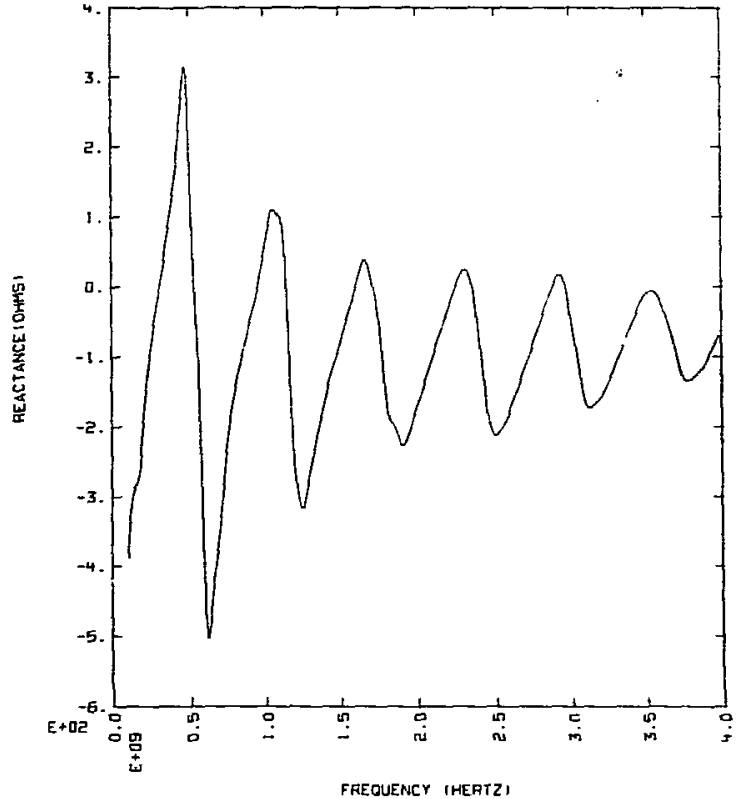

FIG. 5.6f. Imaginary part of antenna impedance, Im $\left(z_{a}\right)$, as a function of frequency for scale-model 35-ft whip antenna. 


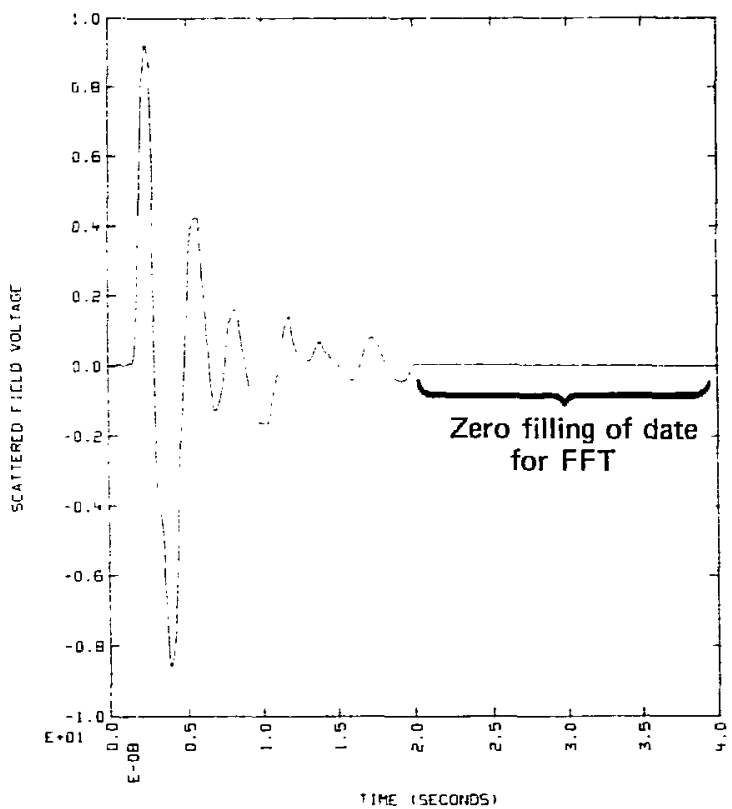

FIG. $5.6 \mathrm{~g}$. Transient response of scale-model antenna due to incident EM pulse.

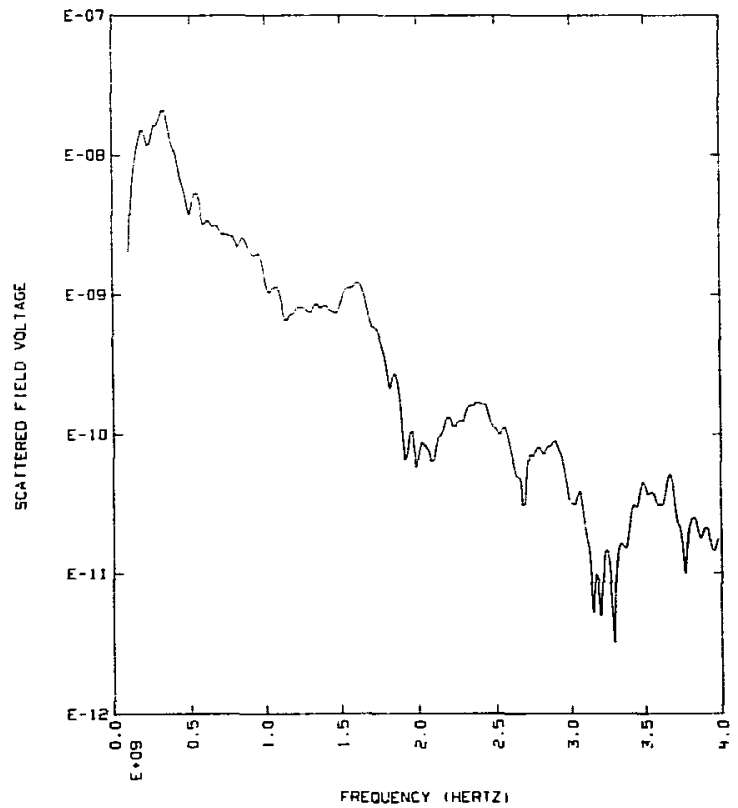

FIG. 5.6h. Fourier transform of scale-model transient response. 


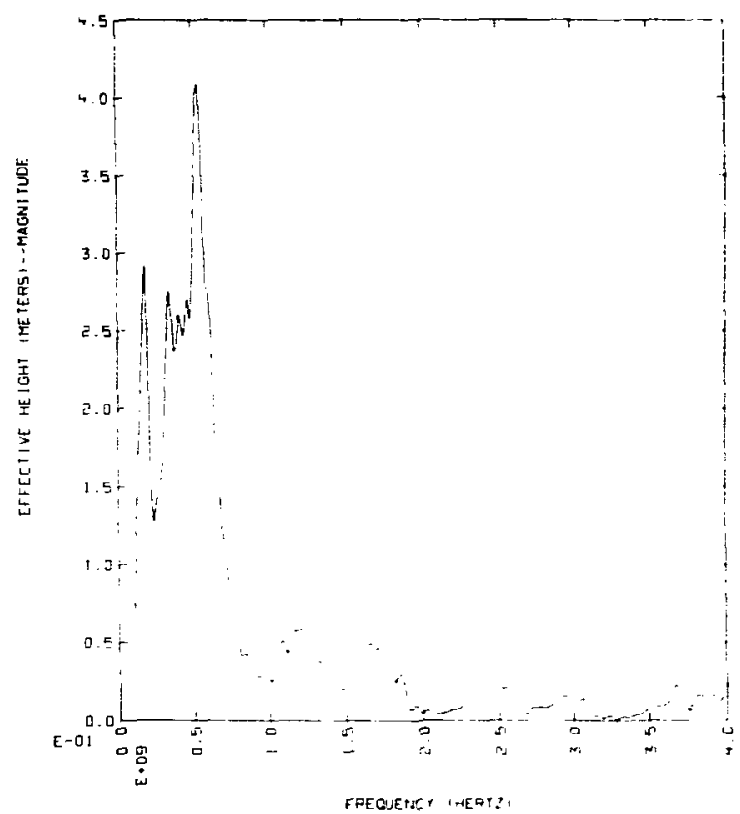

FIG. 5.6i. Scale model effective height, $H_{\text {eff }}$ (f), as a function of E requency.

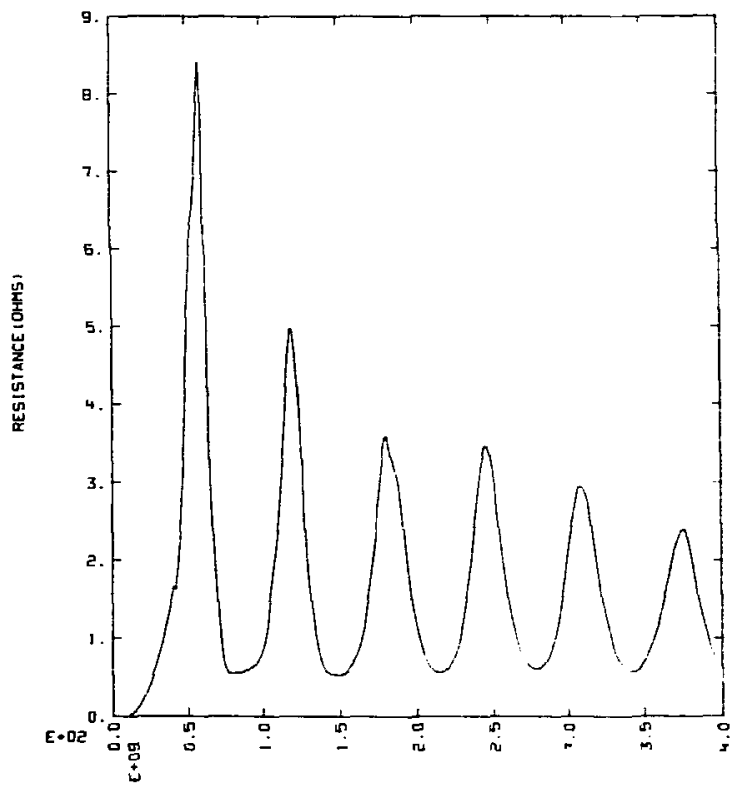

FFECUERSY IHERTZI

FIG. 5.7a. Real part of antenna input impedance of the MF/HF transmit 35-ft whip (starboard). 


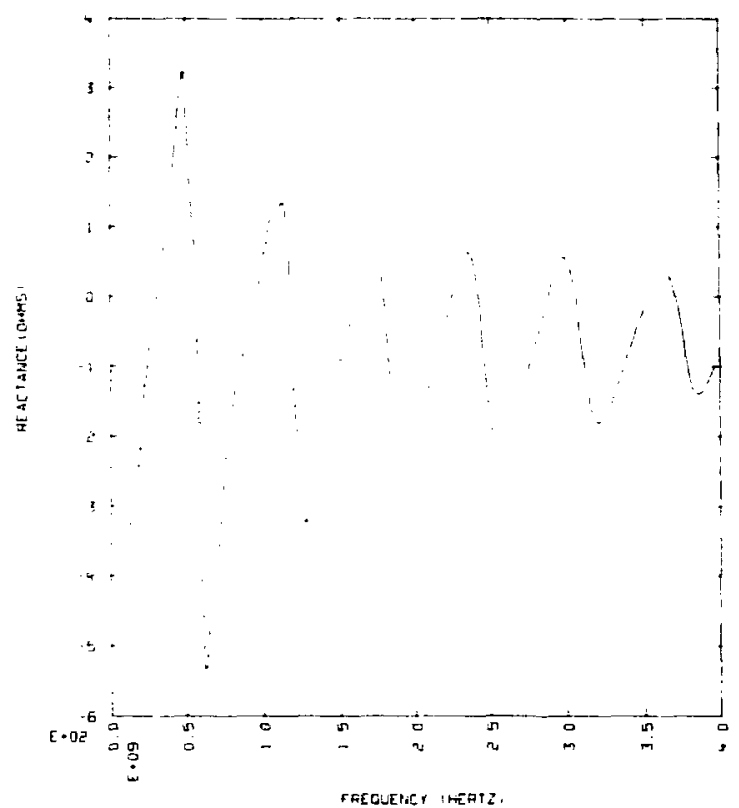

FIG. 5.7b. Imaginary part of antenna input impedance of 35-ft whip (starboard).

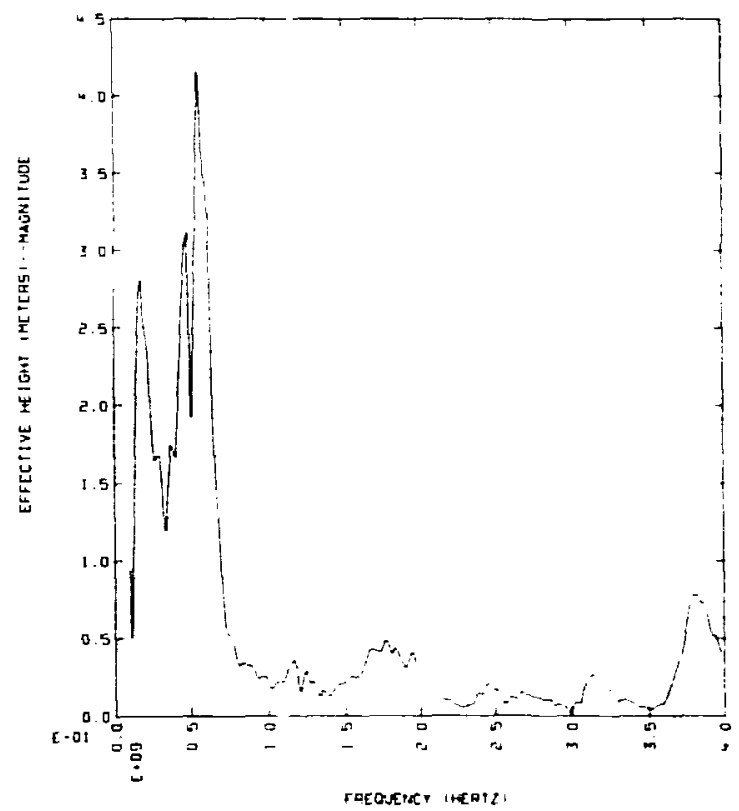

FIG. 5.7c. Effective height of MF/HF transmit 35-ft whip (starboard). 


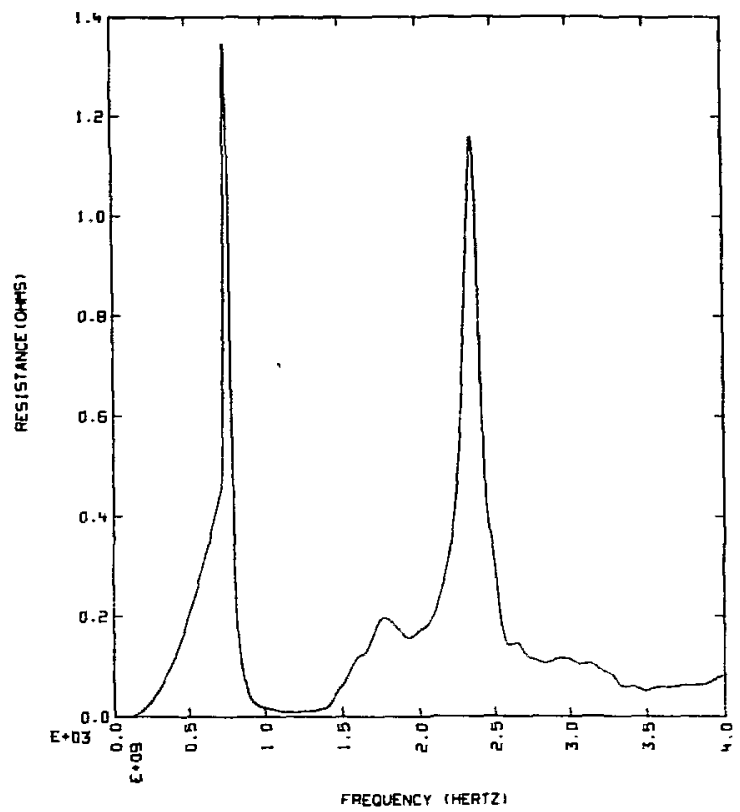

FIG. 5.8a. Real part of antenna input impedance of HF receive fan (port).

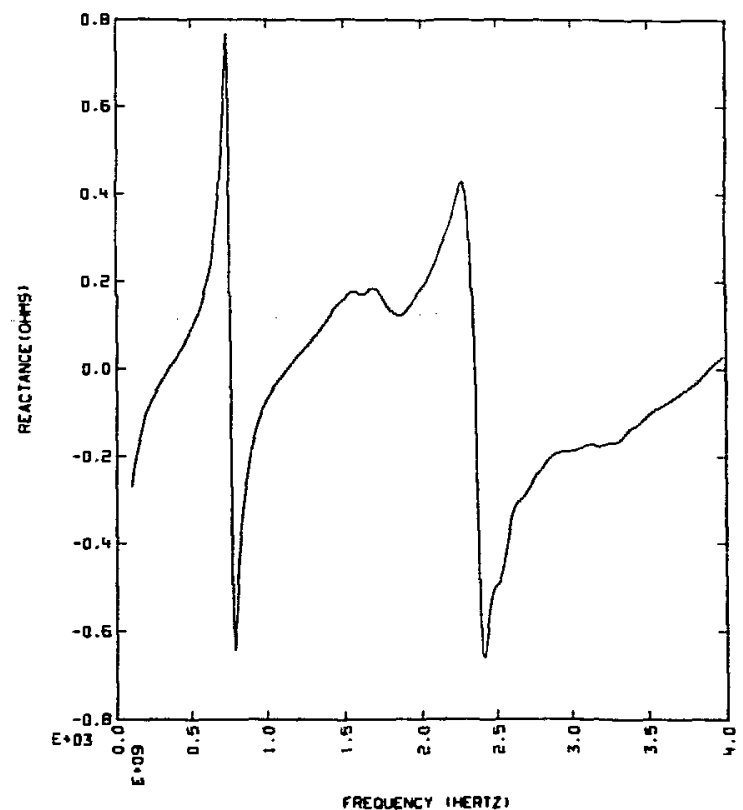

FIG. 5.8b. Imaginary part of antenna impedance of tF receive fan (port). 


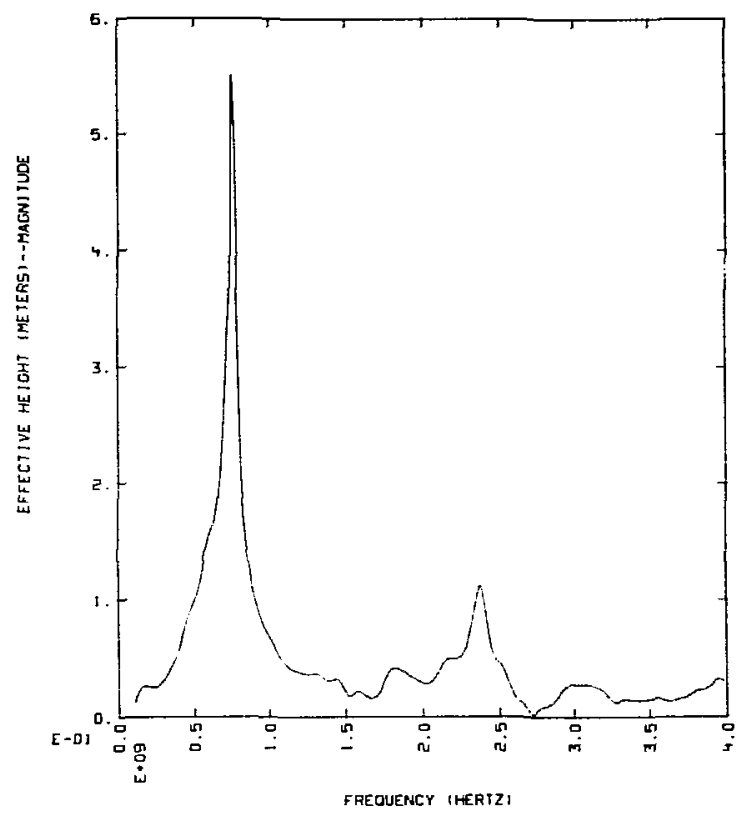

FIG. 5.8c. Effective height of HF receive fan (port).

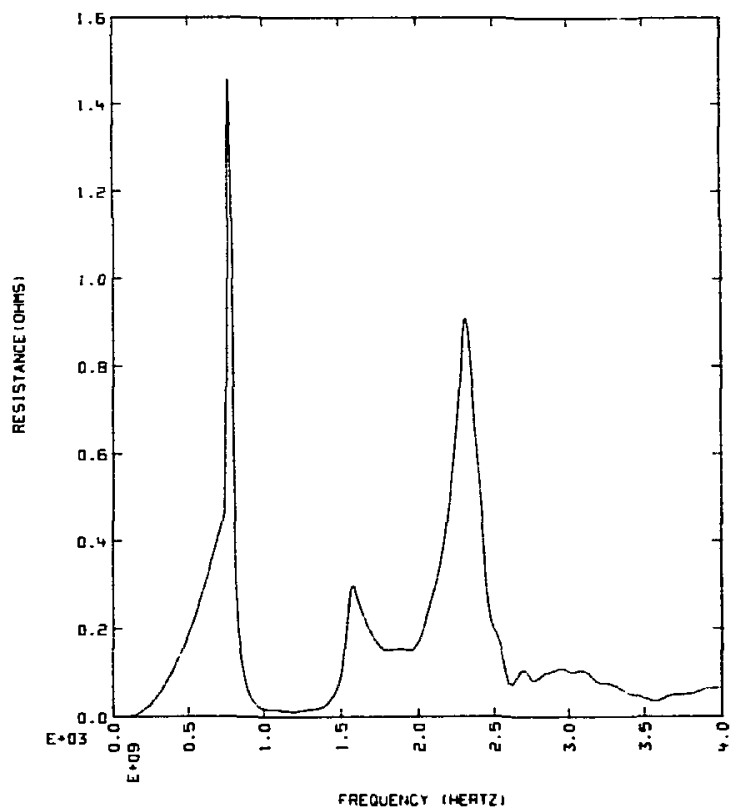

FIG. 5.9a. Real part of antenna input impedance of HF receive fan (starboard). 


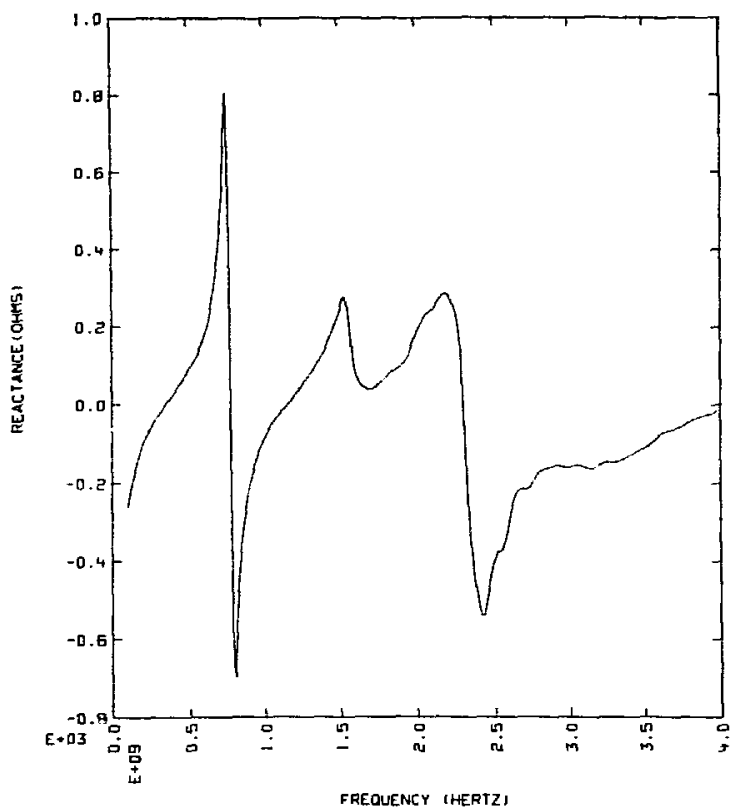

FIG. 5.9b. Imaginary part of HF receive fan input impedance (starboard).

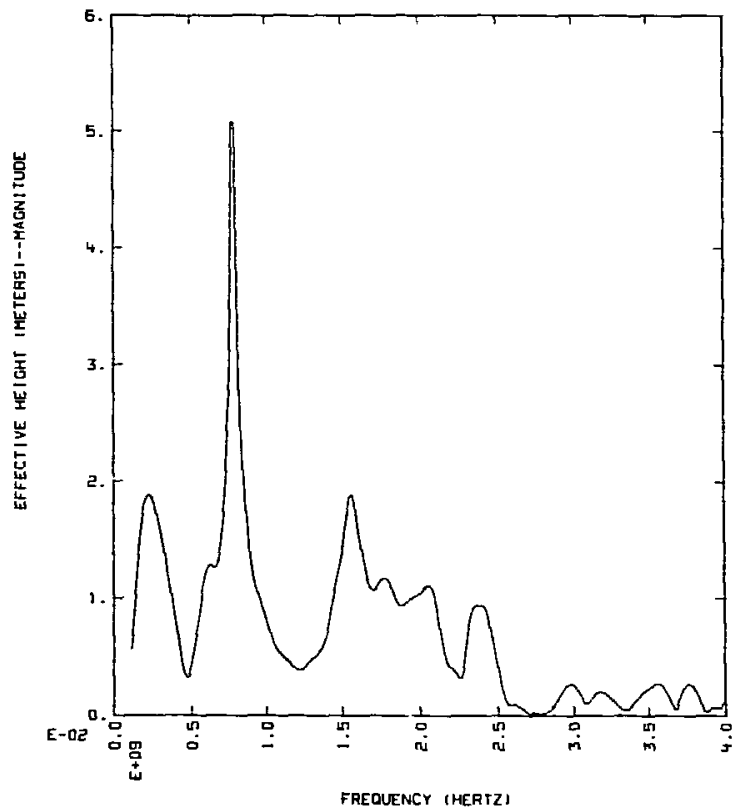

FIG. 5.9c. Effective height of HF receive fan (starboard). 


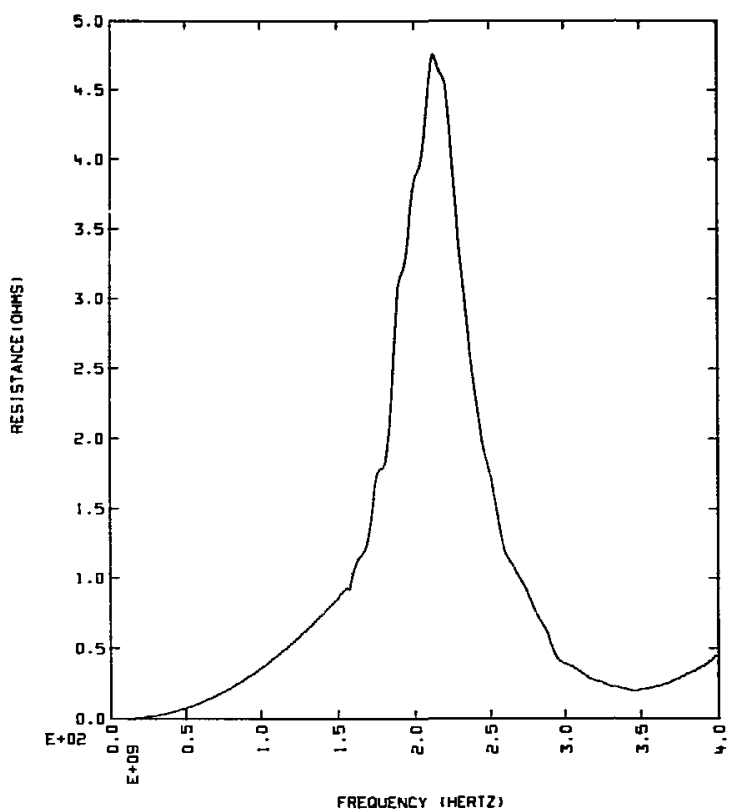

FIG. 5.10a. Real part of $\mathrm{z}_{\mathrm{a}}$ of the AN/SRN-12 Omega antenna.

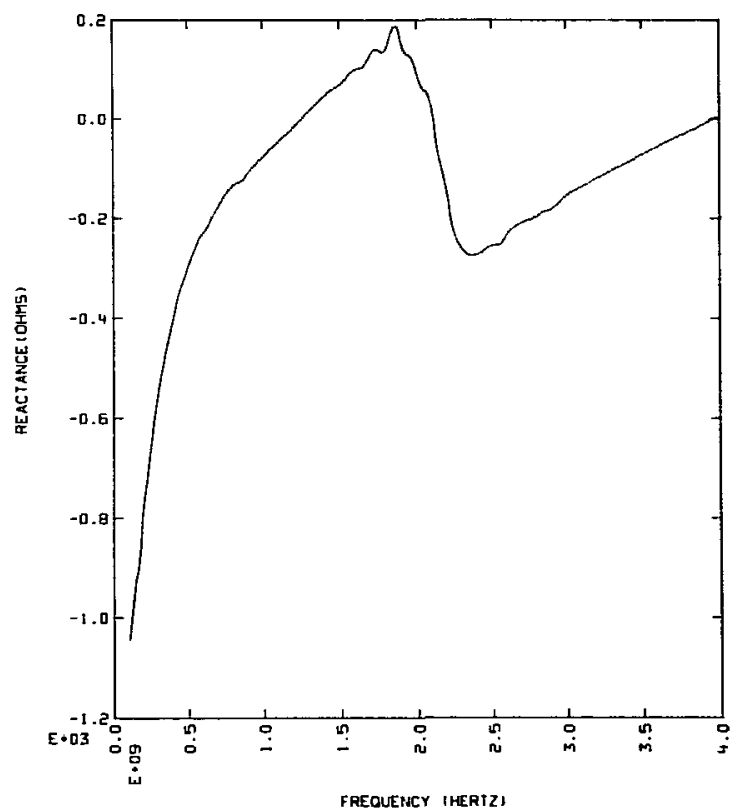

FIG. 5.10b. Imaginary part of $\mathrm{z}_{\mathrm{a}}$ of the AN/SRN-12 Omega antenna. 


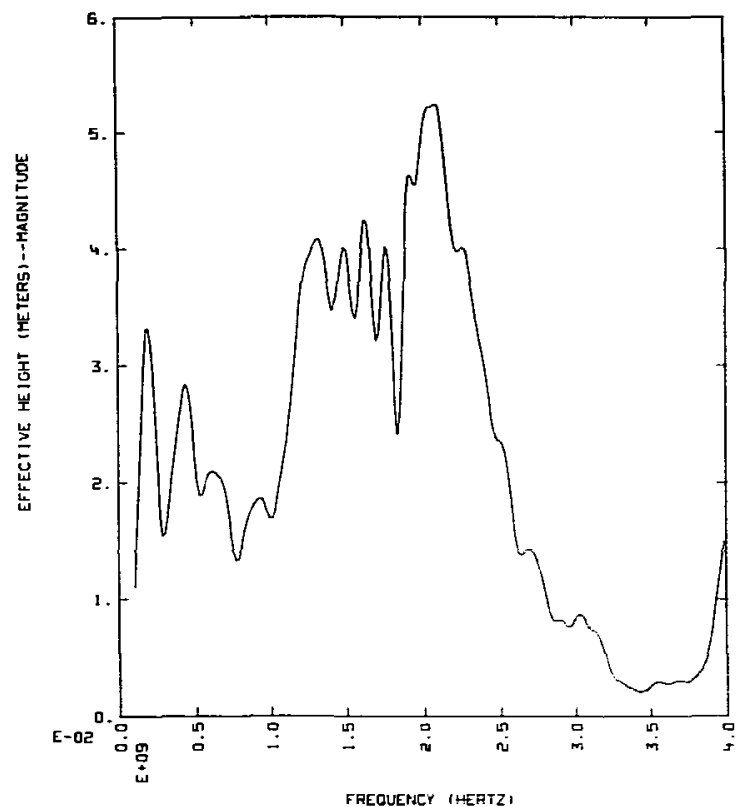

FIG. 5.10c. Effective height of the AN/SRN-12 Omega antenna.

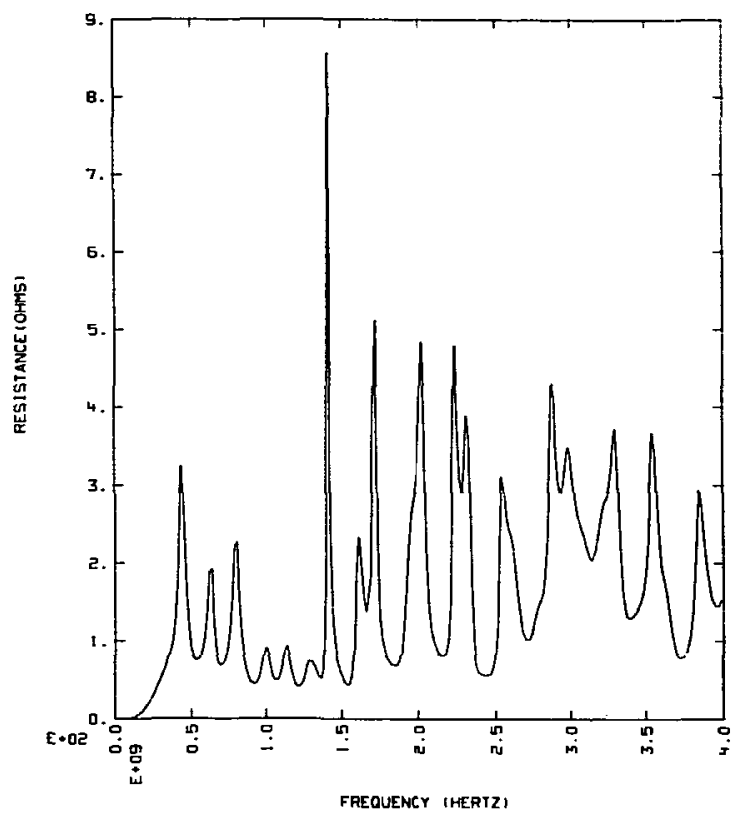

FIG. 5.1la. Real part of $z_{a}$ of the AN/SRC-23 antenna (starboard). 


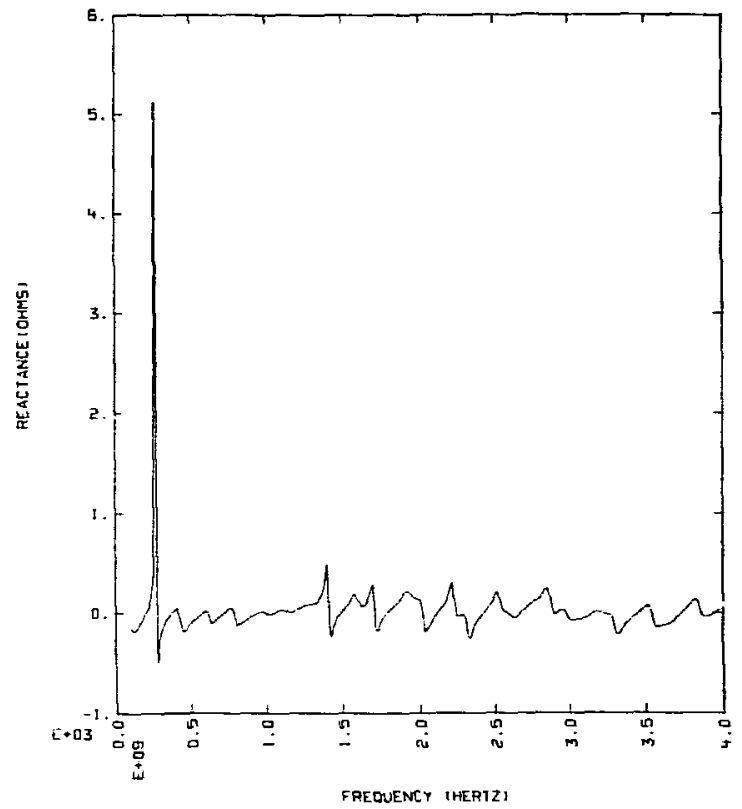

FIG. 5.1lb. Imaginary part of $z_{a}$ of the AN/SRC-23 antenna (starboard).

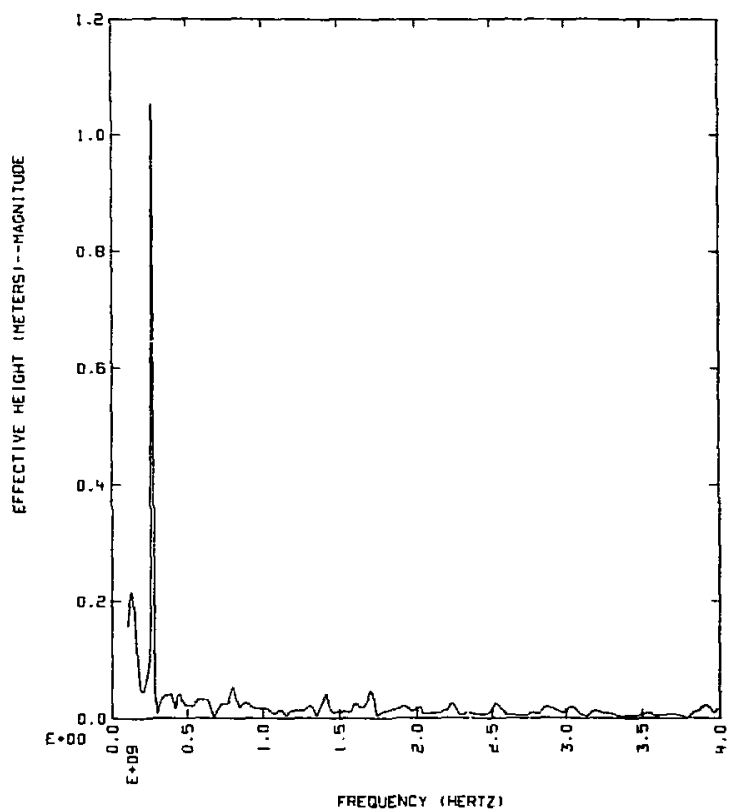

FIG. 5.1lc. Effective height of AN/SCR-23 antenna (starboard). 


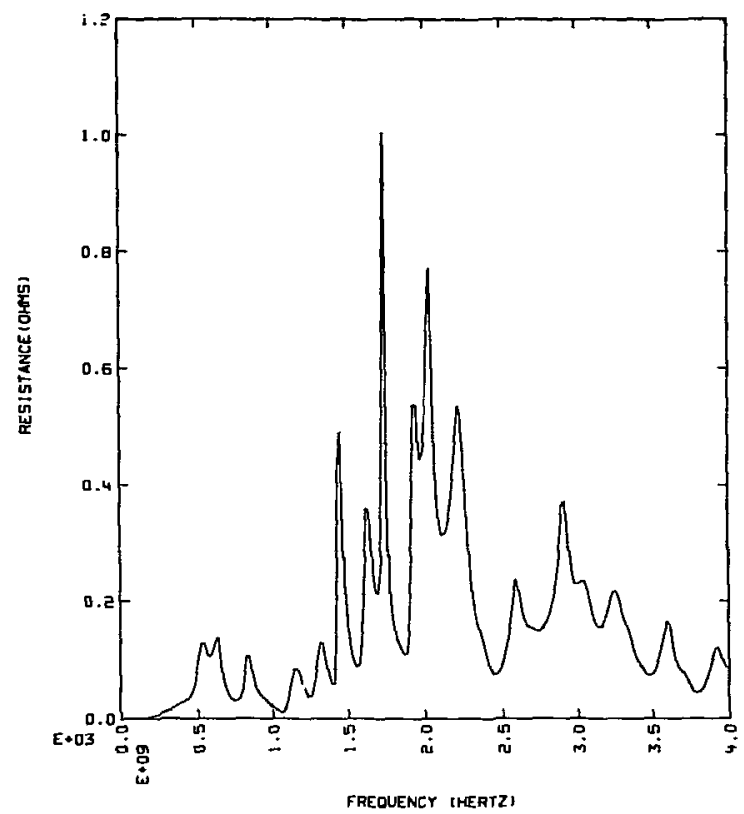

FIG. 5.12a. Real part of $z_{a}$ of the AN/SRC-23 antenna (port).

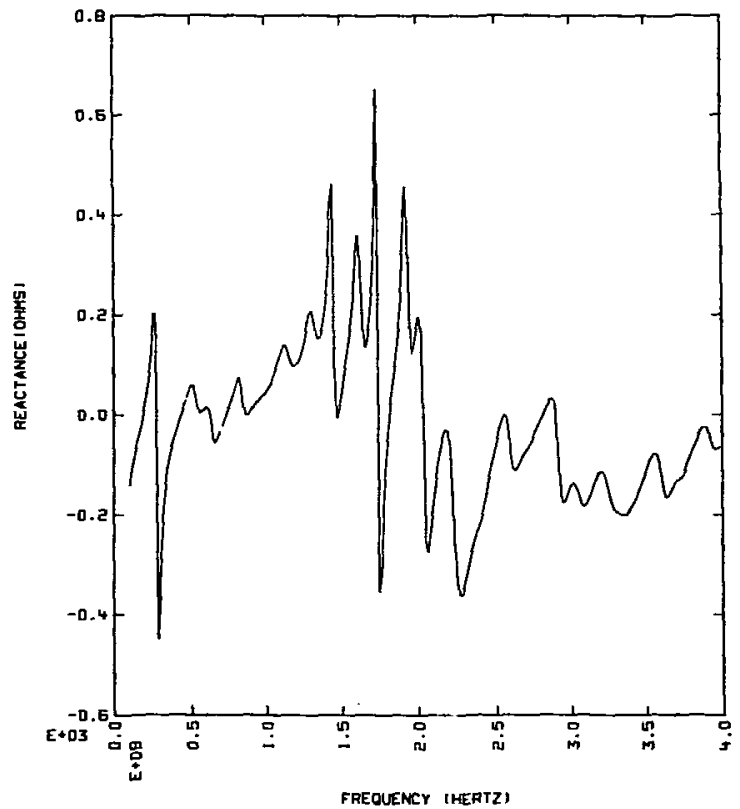

FIG: 5.12b. Imaginary part of $z_{a}$ of the AN/SRC-23 antenna (port). 


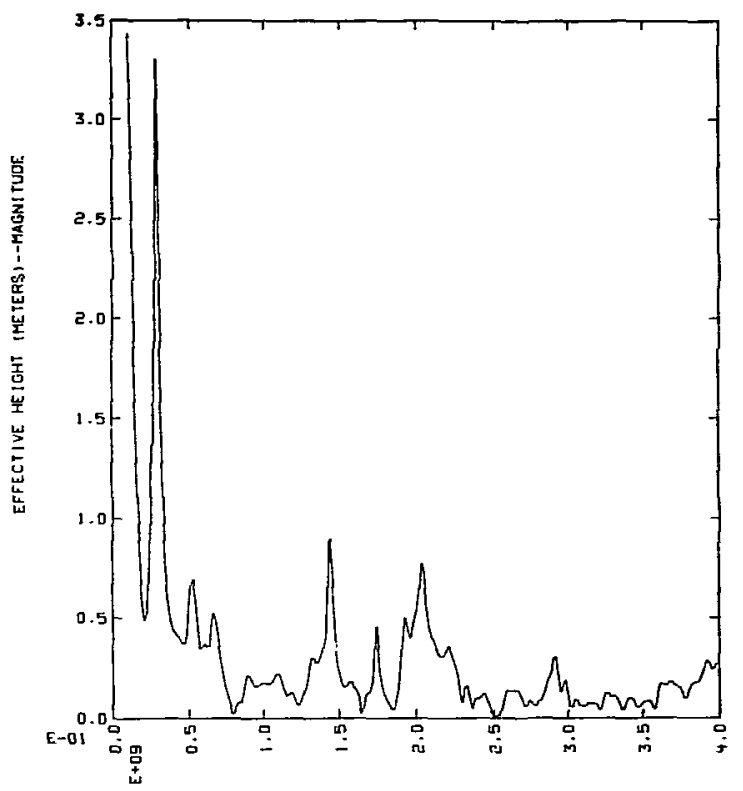

FREDUENCY IHERTZ)

FIG. 5.12c. Effective height of the AN/SRC-23 (port).

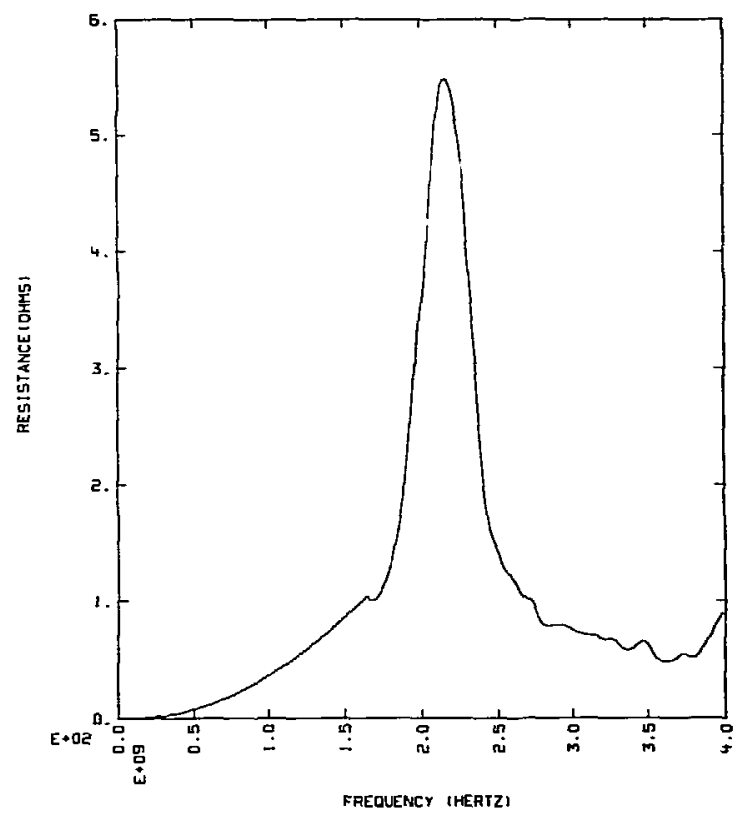

FIG. 5.13a. Real part of $z_{a}$ of the AT-924B/AN/SRA-17 antenna (port). 


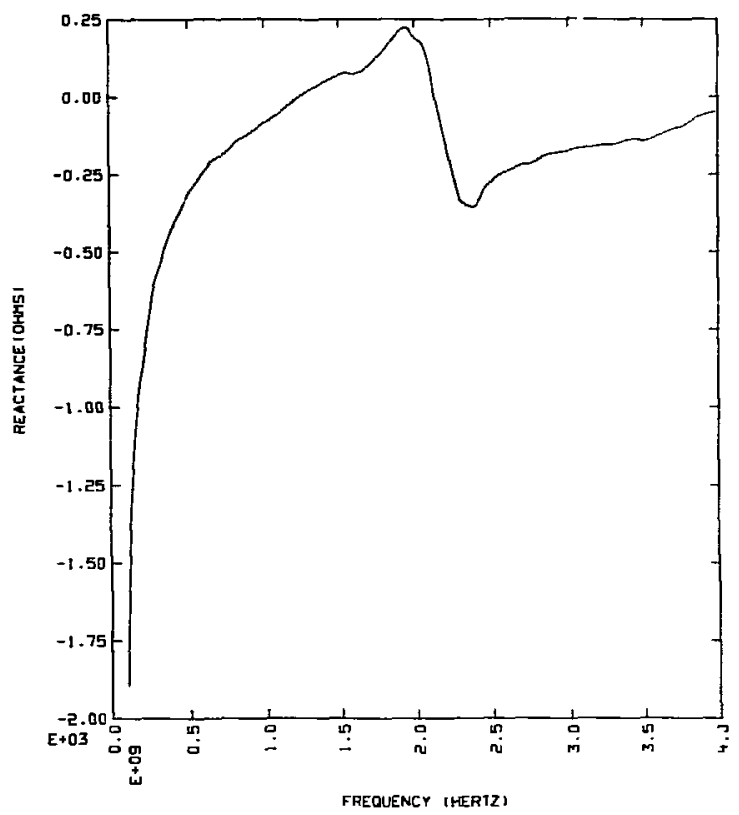

FIG. 5.13b. Imaginary part of $\mathrm{z}_{\mathrm{a}}$ of the AT-924B/AN/SRA-17c antenna (port).

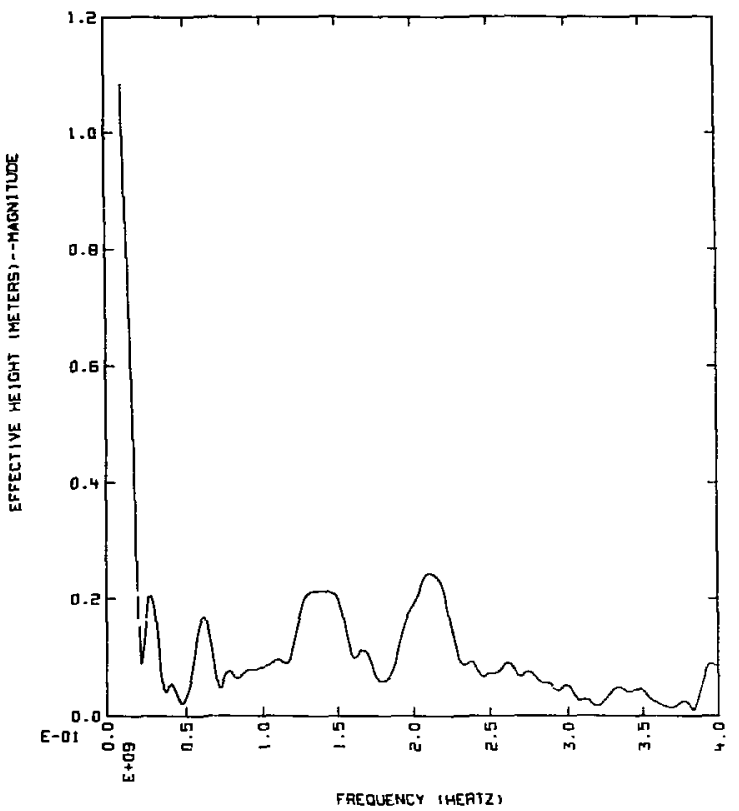

FIG. 5.13c. Effective height of the AT-924B/AN/SRA-17C antenna (port).

5-23 


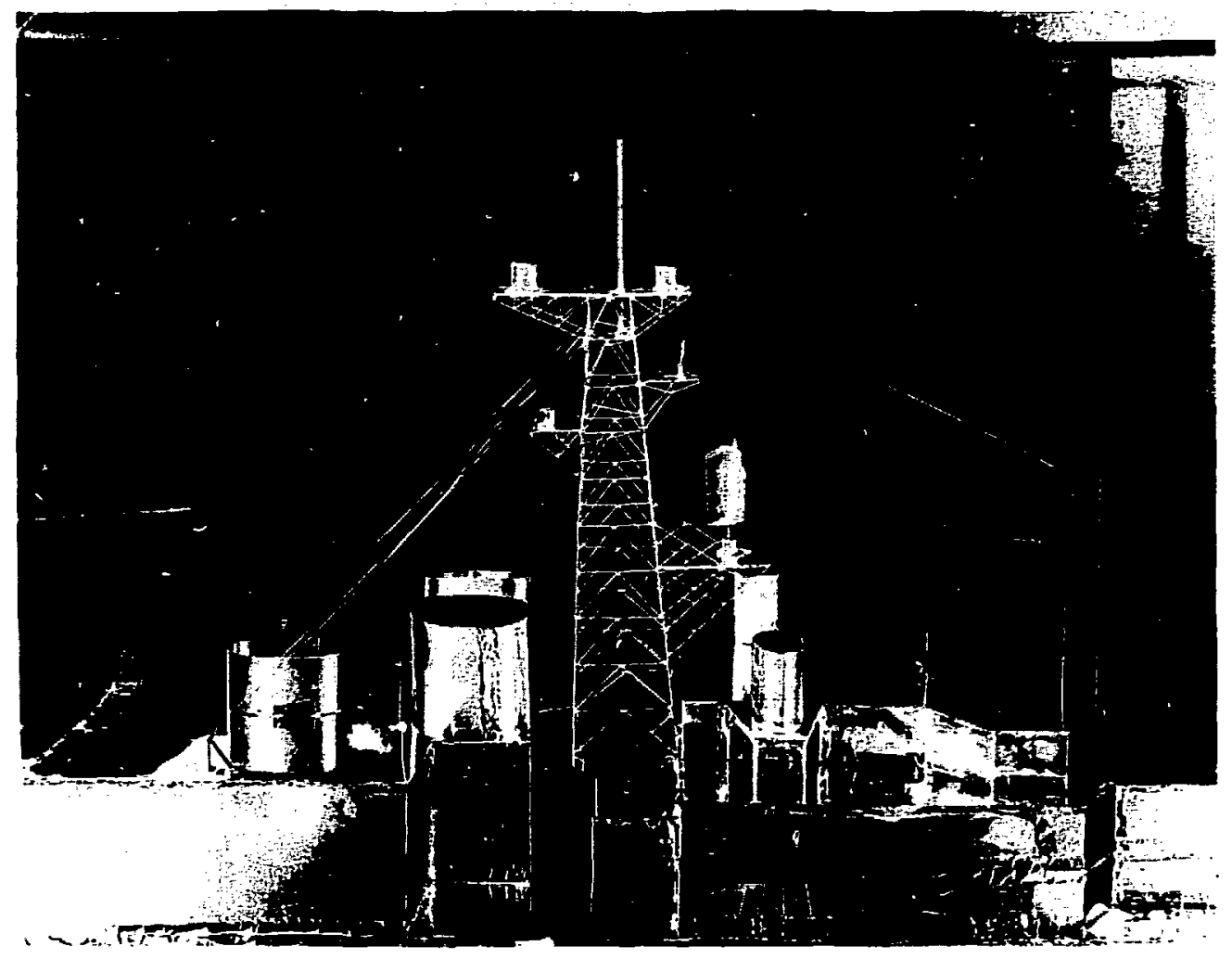

FIG. 5.14. Close-up of mast section of Huron model. 


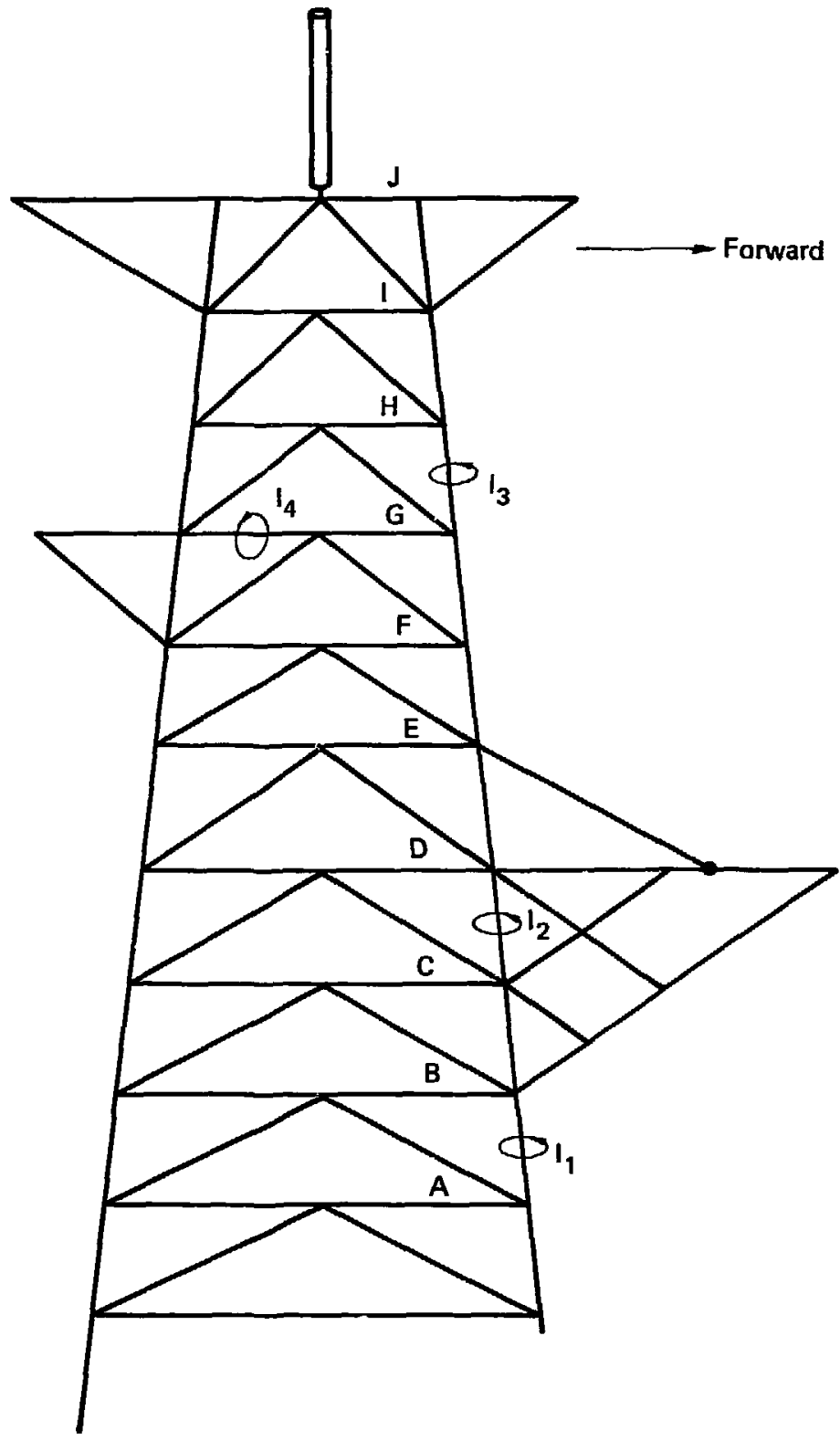

FIG. 5.15. Current measurement points on main mast.

5-25 


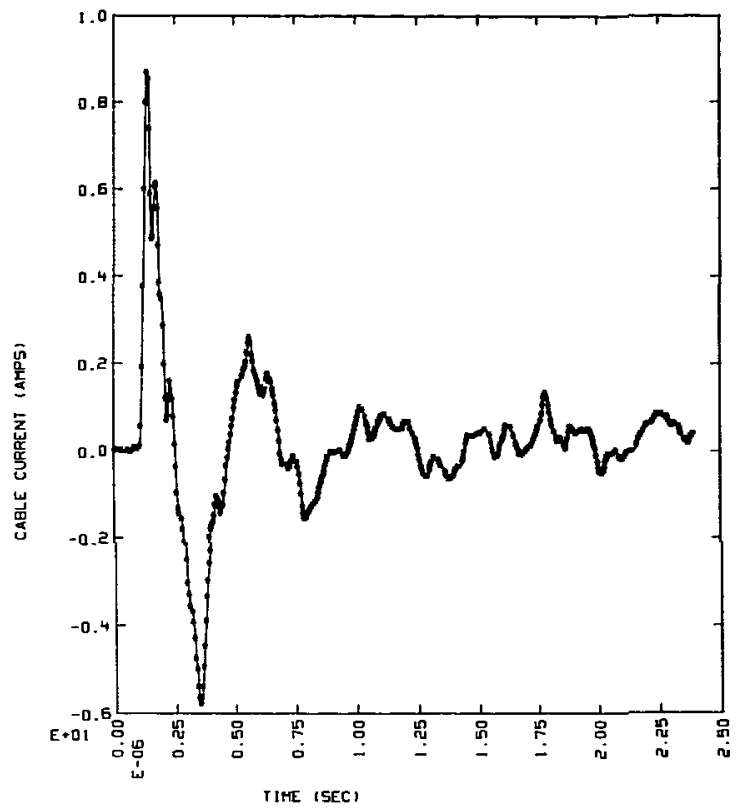

FIG. 5.16a. Temporal mast current between levels A and B.

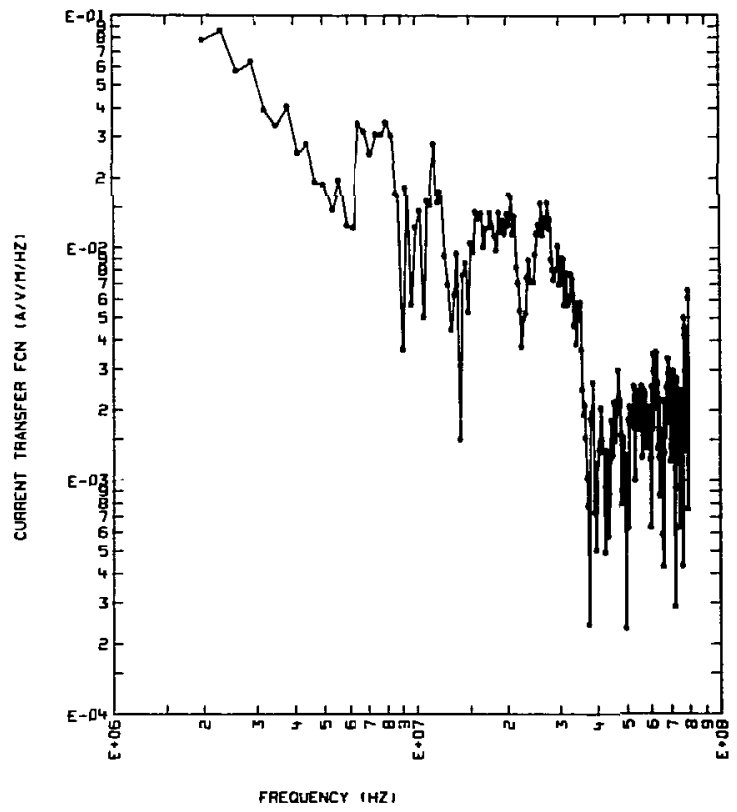

FIG. 5.16b. Mast current spectrum between levels A and B.

5-26 


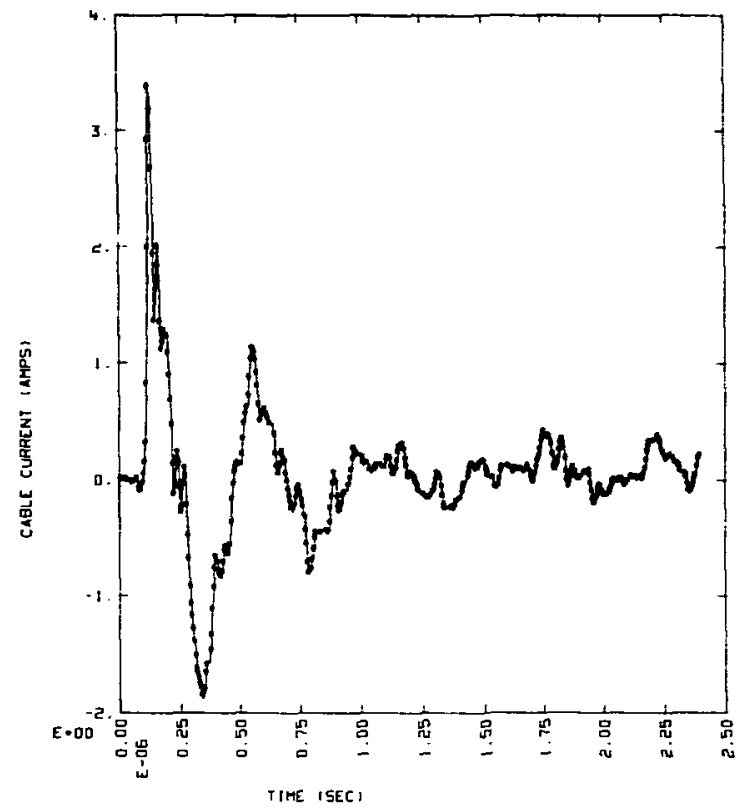

FIG. 5.17a. Temporal mast current between levels $C$ and $D$.

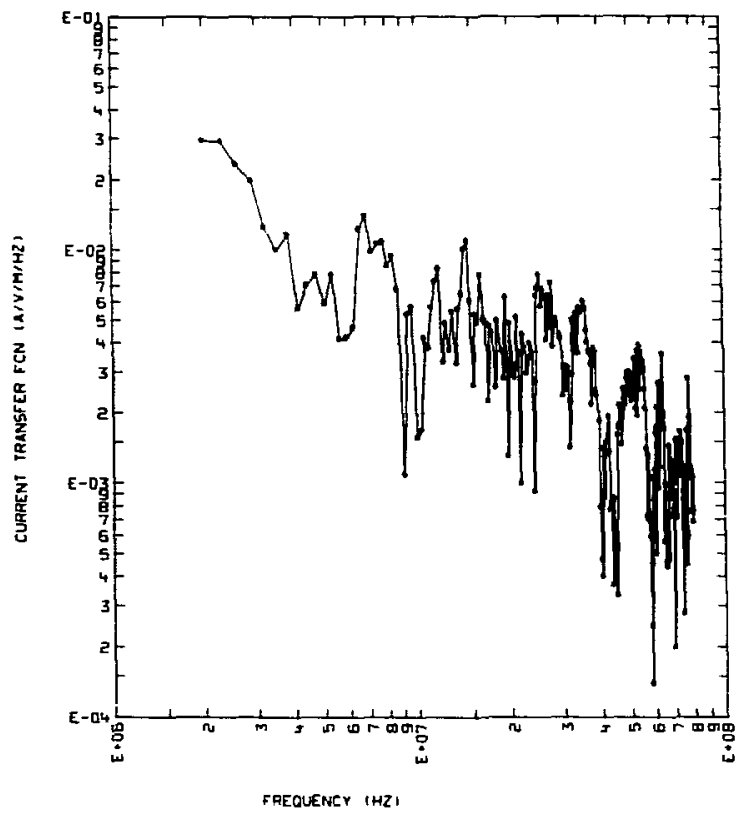

FIG. 5.17b. Mast current spectrum between levels $C$ and $D$. 


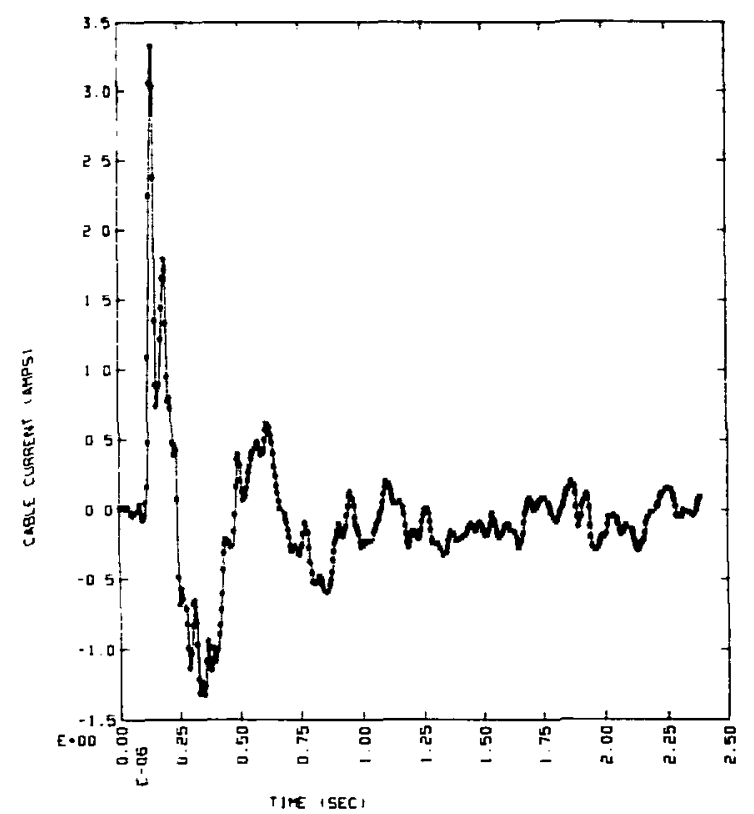

FIG. 5.18a. Temporal mast current between levels $G$ and $H$.

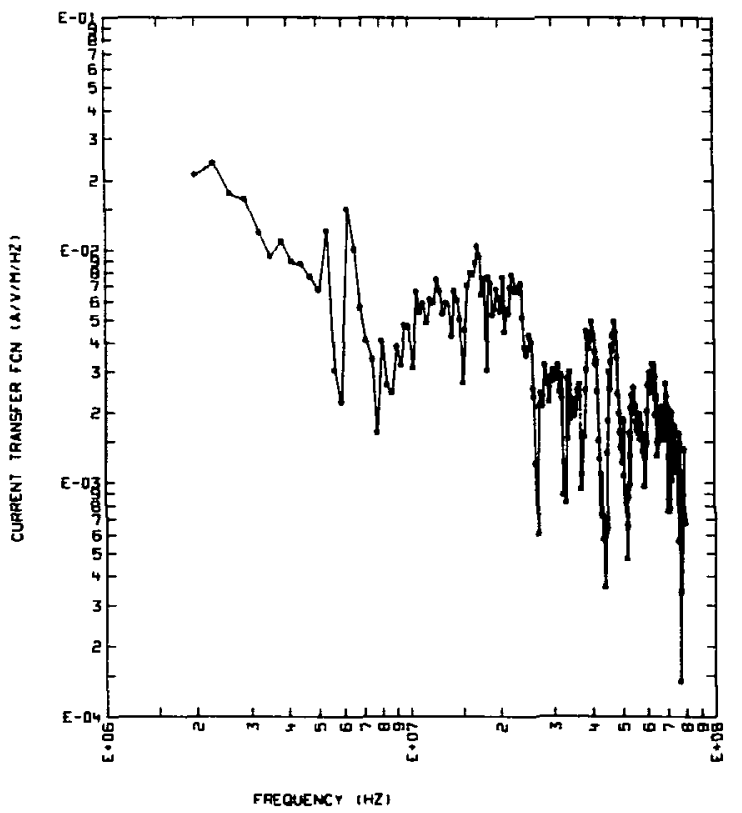

FIG. 5.18b. Mast current spectrum between levels $G$ and $H$. 


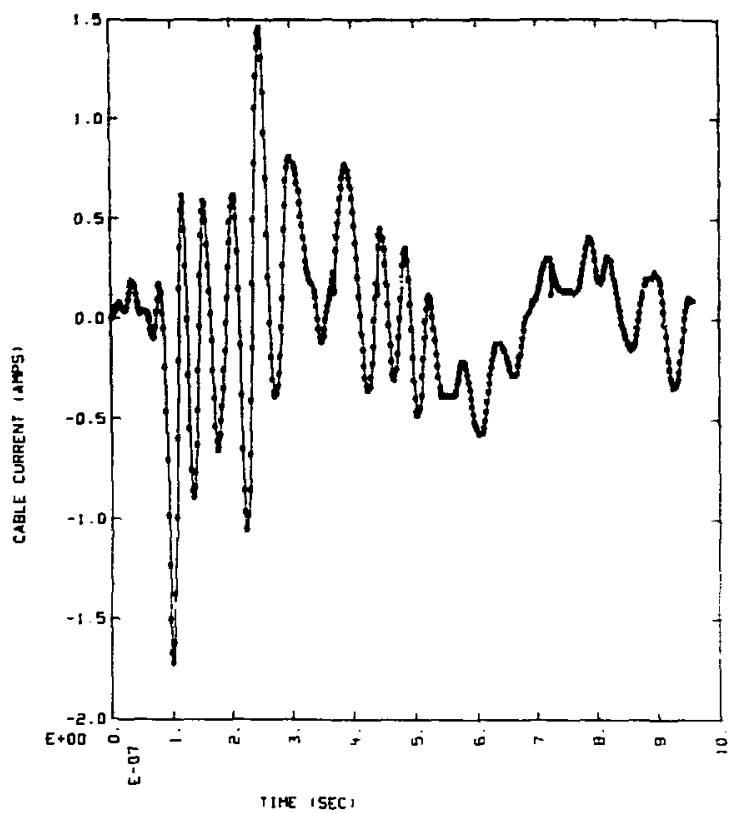

FIG. 5.19a. Temporal current on horizontal strut at mast level G.

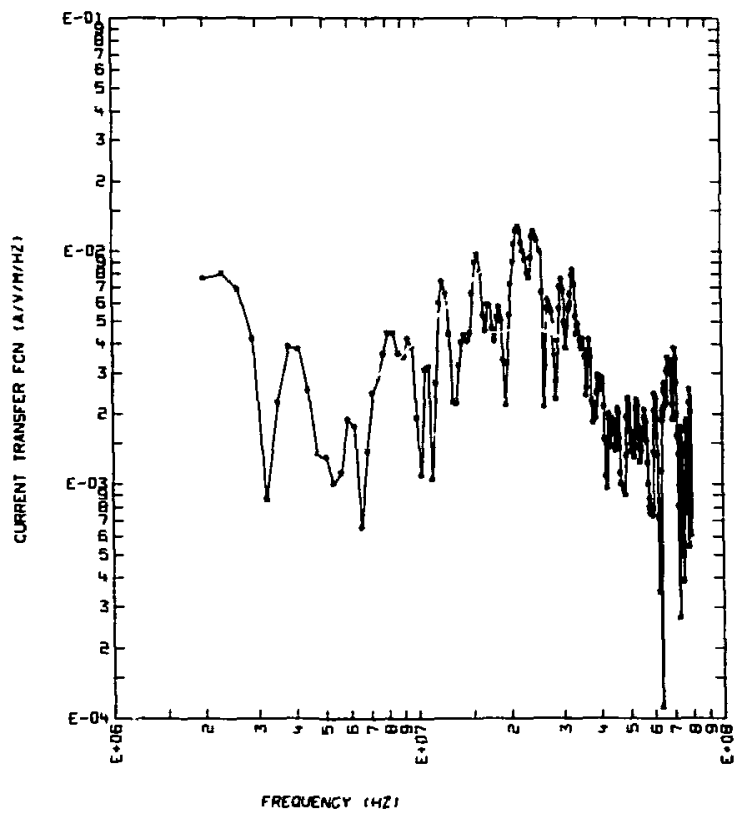

FIG. 5.19b. Current spectrum on horizontal strut at mast level $\mathbf{G}$.

5-29 


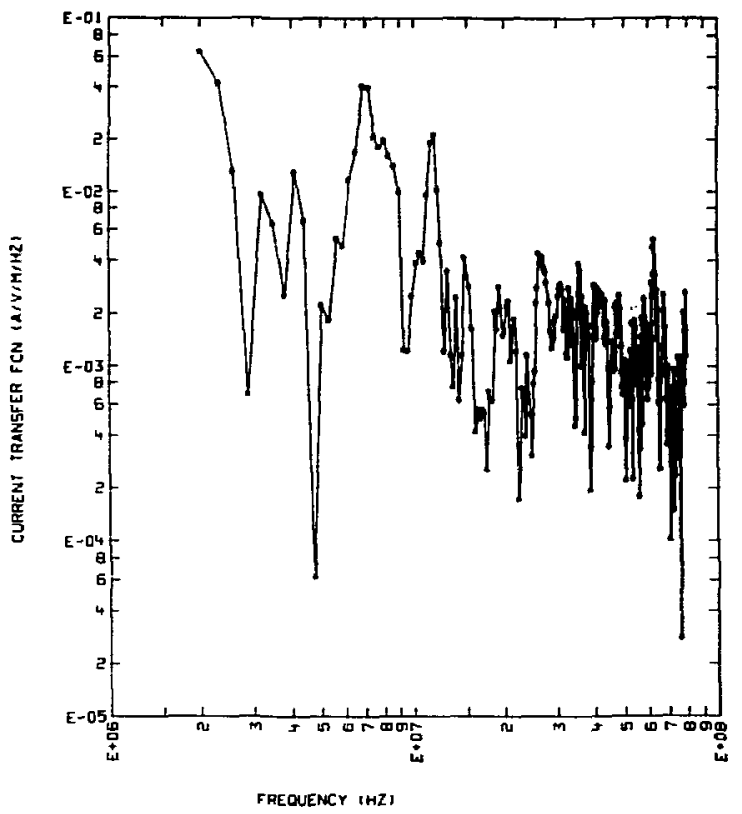

FIG. 5.20a. ENER bulk-cable current transfer function spectrum.

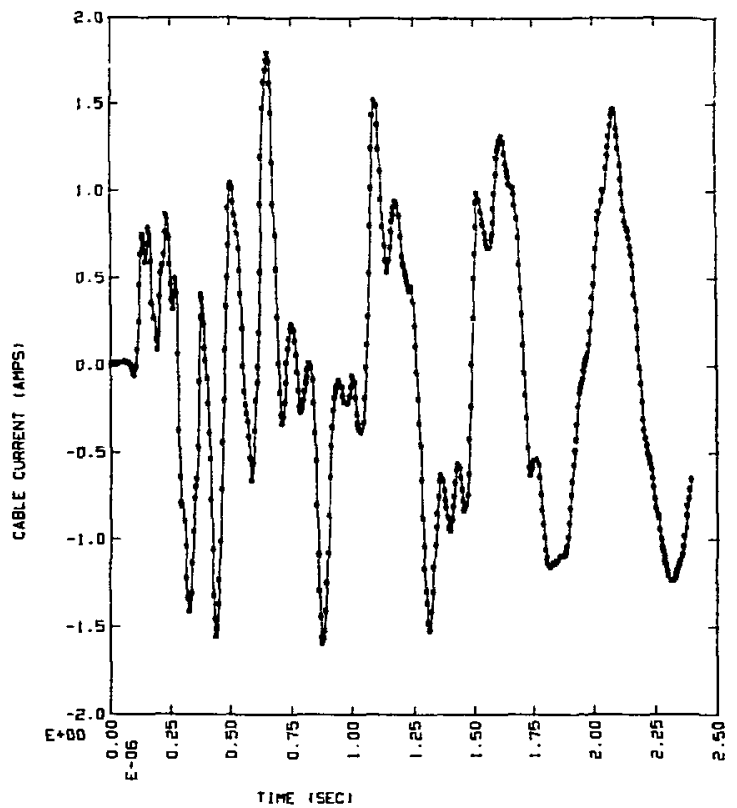

FIG. 5.20b. EWER bulk-cable current. 


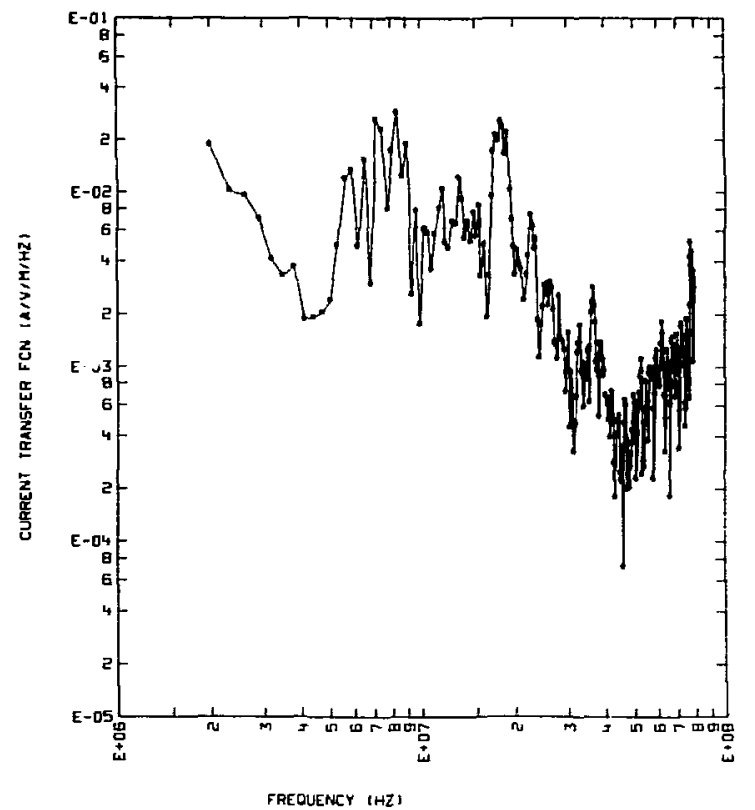

FIG. 5.21a. EnCR bulk-cable current transfer function spectrum.

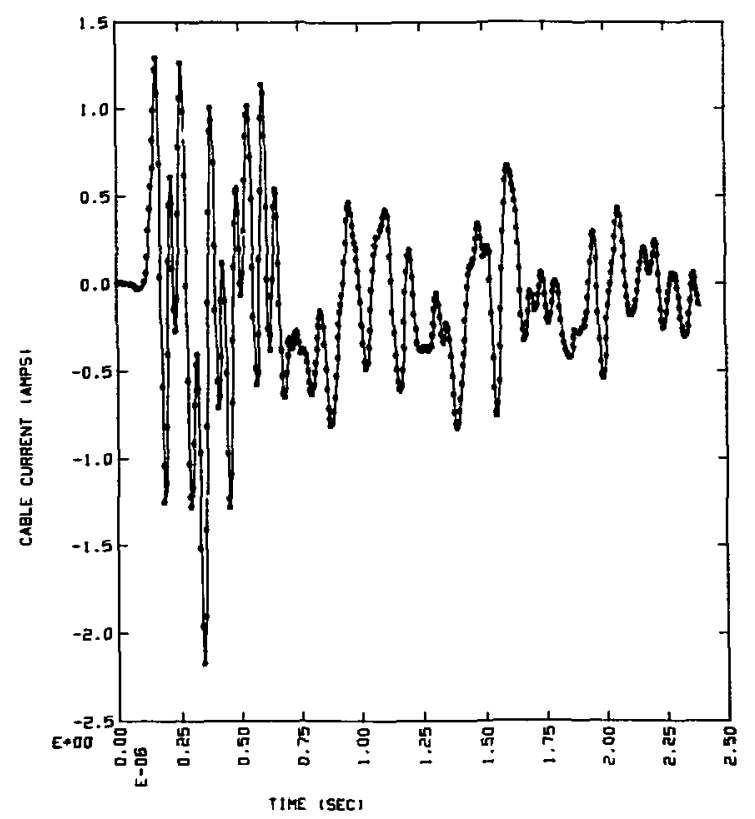

FIG. 5.21b. EACR bulk-cable current. 


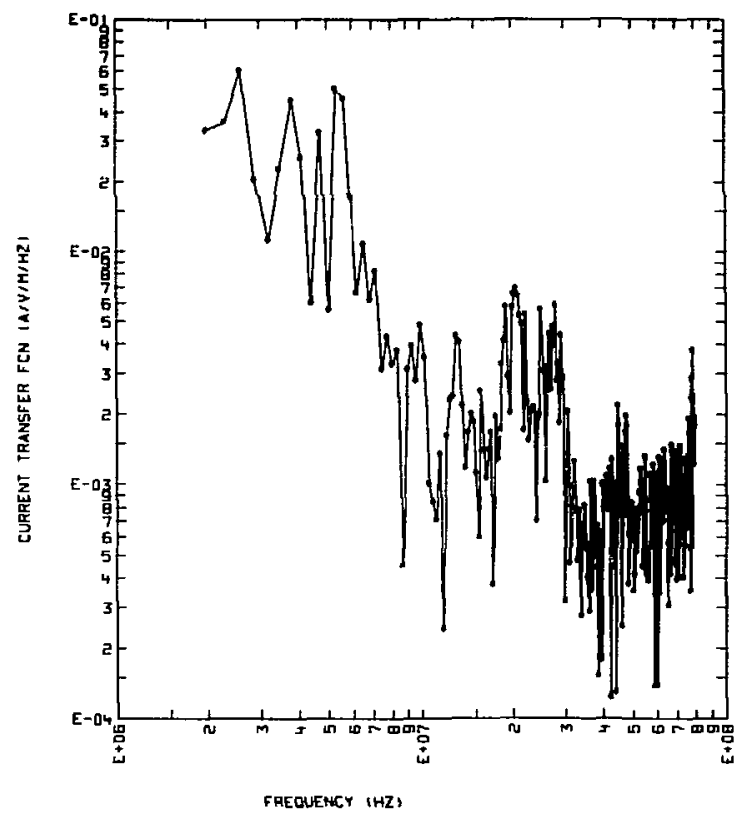

FIG. 5.22a. TACAN bulk-cable current transfer function spectrum.

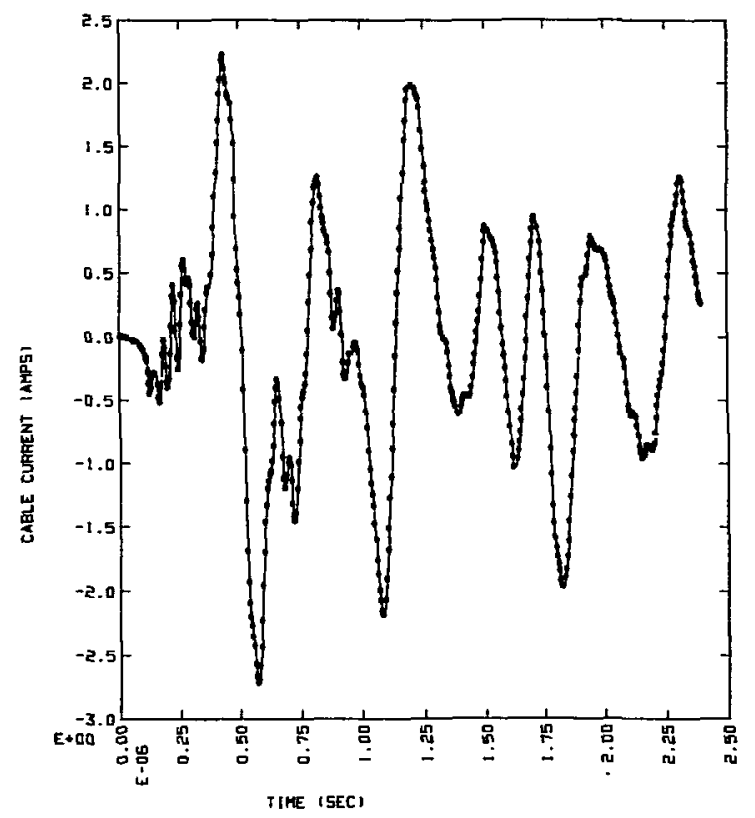

FIG. 5.22b. TACAN bulk-cable current. 


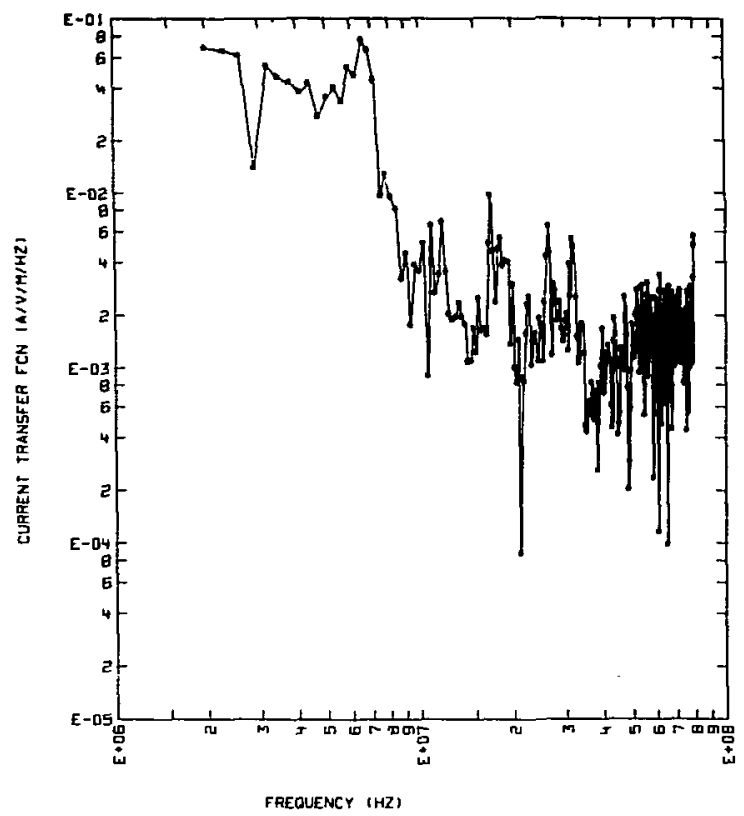

FIG. 5.23a. TX-1 bulk-cable current transfer function spectrum.

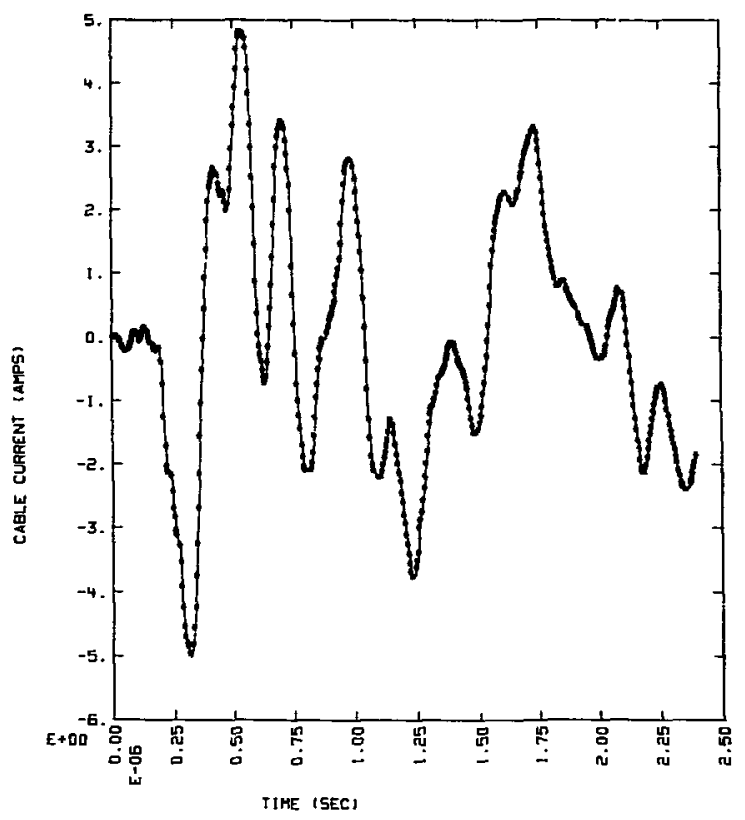

FIG. 5.23b. TX-1 bulk-cable current. 


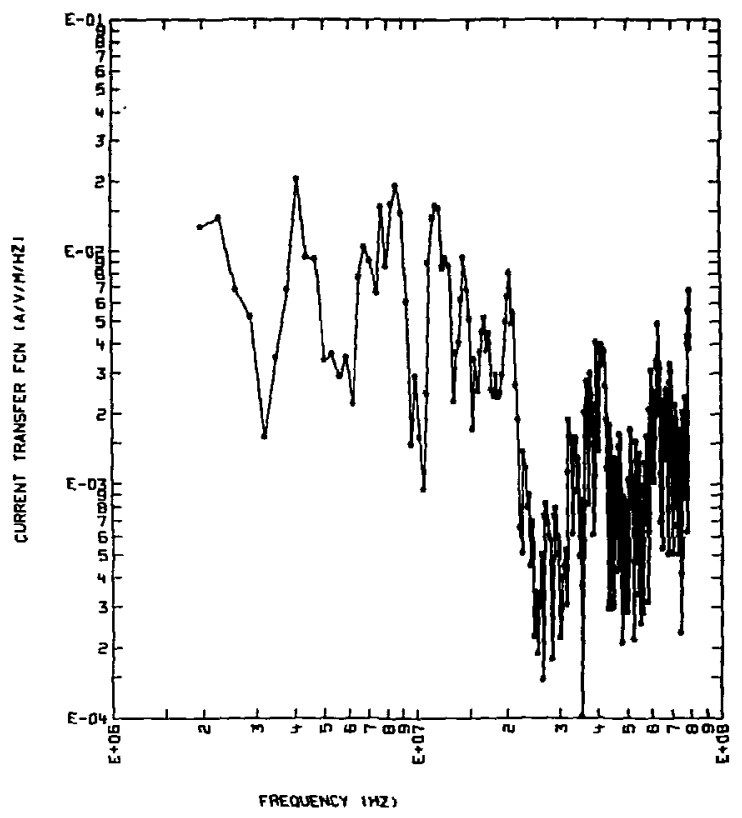

FIG. 5.24a. TX-2 bulk-cable current transfer function spectrum.

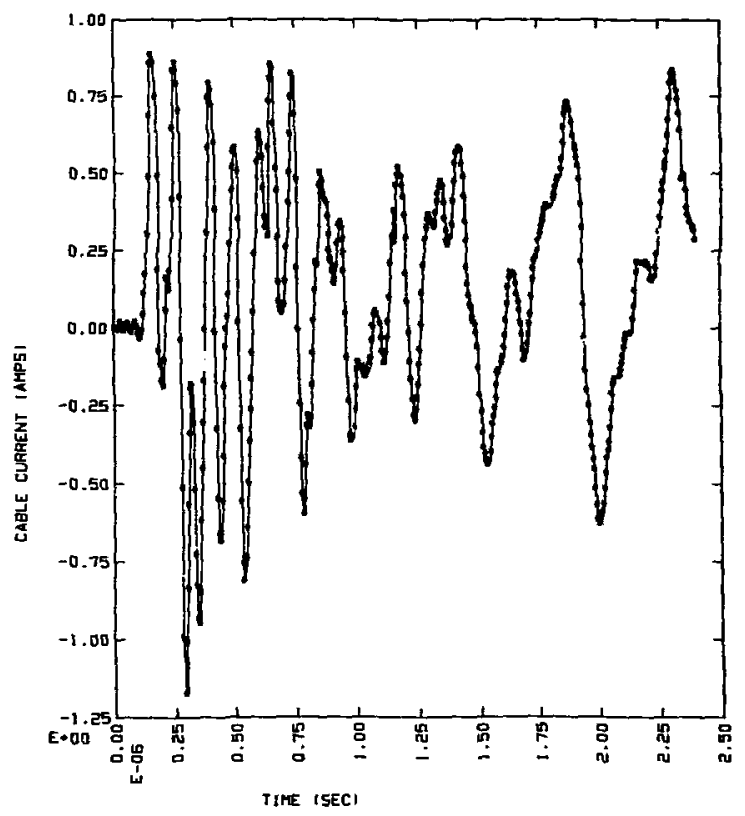

FIG. 5.24b. TX-2 bulk-cable current. 


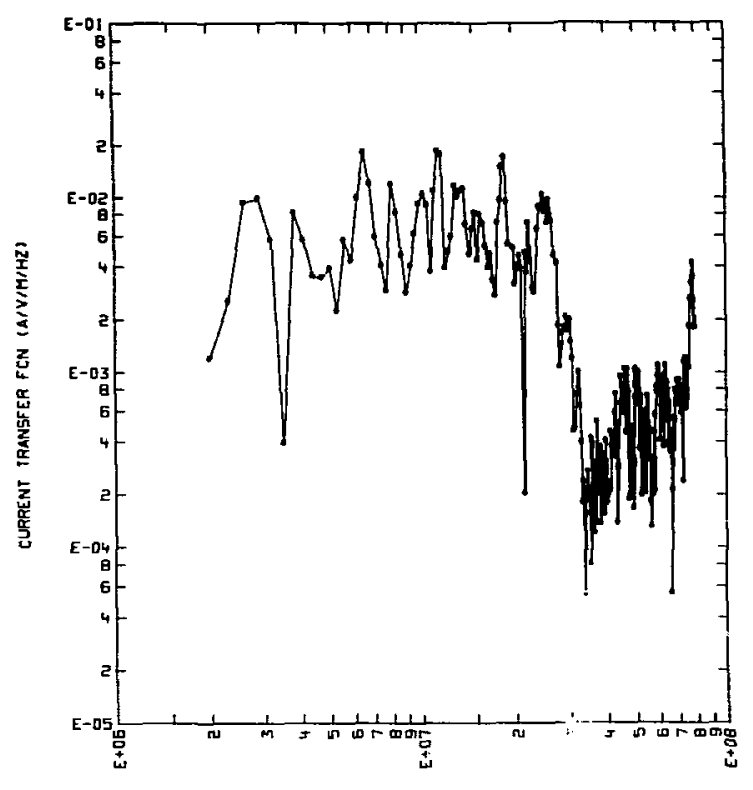

FAEQUENCY (HZ)

FIG. 5.25a. CCR bulk-cable current transfer function spectrum.

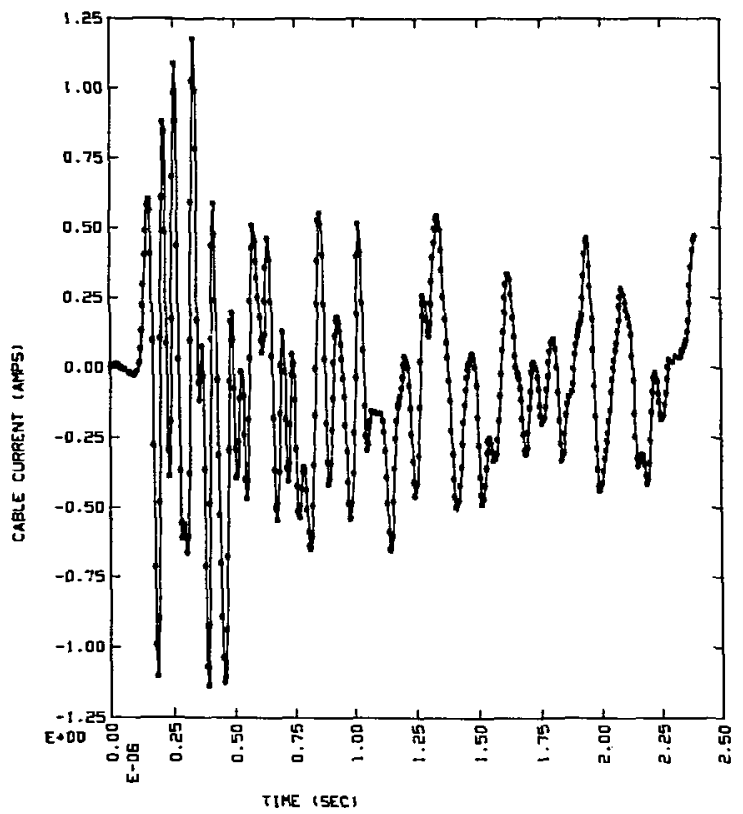

FIG. 5.25b. CCR bulk-cable current. 
$5-36$ 


\subsection{PROCESSED DATA}

We obtained data from the LLL transient range for a 1/48-scale model of the Huron illuminated by the impulse EM field from the IKOR/Monocone source. To compare our scale-model measurements with the full-scale tests conducted by NSWC, it was necessary to convolve our measured transfer function with the field produced by the EMPRESS facility. This section describes how the model data were processed and presents the scaled-up results in both the time and frequency domains.

\subsection{EMERESS FIELDS}

The full-scale tests conducted on the Huron were performed at the U.S. Navy's EMPRESS test facility in Maryland. Figure 6.1 is a map of this facility. While measurements were taken for both horizontal and vertical polarizations, the scale model measurements were only made for a vertical polarization. Figure 6.2 shows the time-domain vertical electric field measured at the ship anchor point. The spectrun of the waveform is shown in Fig. 6.3.

For our calculations, it was necessary to first digitize the time pulse waveform and then Fourier transform this digitized pulse to the frequency domain. Figure 6.4 shows the resulting digitized waveform, and Fig. 6.5 shows the transform. We obtained good agreement between NSWC's spectrum and the spectrum derived from our digitized waveform. The spectrum shown in Fig. 6.5 is used for all our scaling calculations.

\subsection{DATA PROCESSING TECHNIQUES}

This section describes how the model data are scaled-up to full-scale EMPRESS field levels. Different techniques were used for processing the external and internal coupling measurements.

For each of the external antennas on the Huron model we found an equivalent circuit representation like that shown in Fig. 5-5. The parameters of the equivalent model can be easily scaled up to any sized structure, for example, the effective height, $\mathrm{H}_{\text {eff }}(\mathrm{f})$ is simply multipled by the model size scale factor (48 in this case). The antenna input impedance does not scale with model size; only the frequency scales, and it scales inversely with the 
scale factor. The $\mathrm{E}_{\text {INC }}(f)$ incident field quantity in the equivalent circuit may be any field value which we desire to find the response of the circuit. For this work we used the frequency domain representation of the BHPRESS field, as plotted in Fig. 6.5.

Thus for the external antennas on the ship, we first obtained the equivalent circuit parameters. Then, with the equivalent circuit known, we scaled up the effective height by a factor of 48 , and scaled down the model frequency by the 48 factor. We then used the EMPRESS field spectrum for $\mathrm{E}_{\text {INC }}(\mathrm{f})$ and calculated the following quantities:

$$
\begin{aligned}
& v_{50 \Omega}(f)=H_{E F F}^{\prime}(f) E_{I N C} C_{\text {EMPRESS }} \text { (f) } \frac{50 \Omega}{\left(z_{a}(f)+50 \Omega\right)} \\
& I_{50 \Omega}(f)=H_{E F F}^{\prime} \text { (f) } E_{\text {INC }}{ }_{\text {EMPRESS }} \text { (f) } /\left(z_{a}(f)+50 \Omega\right) \text {; } \\
& v_{O C}(f)=H_{E F F}^{\prime}(f) E_{\text {INC }}{ }_{\text {EMPRESS }}^{(f)} \text {; } \\
& I_{S C}(f)=H_{E F F}^{\prime}(f) E_{I N C}{ }_{\text {FMPRESS }}(f) / Z_{a}(E) .
\end{aligned}
$$

Here $V_{50 \Omega}(f)$ and $I_{50 \Omega}(f)$ are the respective $50 \Omega$ load voltage and the current spectrums. $V_{\alpha c}(f)$ is the open-circuit voltage spectrum and $I_{s c}{ }^{(f)}$ is the short-circuit current spectrum. Note also that $\mathrm{H}_{\mathrm{EFF}}^{\prime}(f)$ is the scaled-up effective height.

Once the frequency domain spectruns are computed, the full-scale, timedomain responses can then be found by taking the inverse Fourier transforms of the spectrums.

The computations for the internal bulk cable currents and tower currents were handled in a slightly different manner. In these cases we only measured the scale-njdel currents and then Fourier transformed the time-domain currents to the frequency domain. This spectrum is then normalized by the spectrum of the incident EM pulse used for the measurement to provide the impulse-response transfer function. To find the response to the EMPREss field, we multiply the impulse-response transfer function by the FupRess spectrum. The resulting current spectrum may then be inverse Fourier transformed to yield the time-domain current waveforms. 
The next two sections present the results obtained from this work. Scalemodel measurements are scaled-up to a full-sized ship and the incident fields are scaled-up to the full-scale EMPREss pulse.

\subsection{FULL-SCALE EXTERNAL COUPLING PREDICTIONS}

The full-scale predictions based on the antennas measured on the ship model are presented here. For each antenna we will show the 50-ohm load voltage and current in both the frequency domain and in the time domains. We also show the open-circuit voltage spectrums, the short-circuit current spectrums, and their respective time-domain equivalents.

The order of presentation of the external coupling data is the same as shown earlier in Table 1.1, where the MF/HF TX 35-ft whips on the port and starboard sides are shown first in Figs. 6.6a through 6.7h. The HF receive fans are shown next in Figs. 6.8a through $6.9 \mathrm{~h}$, followed by the port and starboard AN/SRC-23 flat top antennas in Figs. 6.10a through 6.11h. Figures 6.12a through $6.12 \mathrm{~h}$ show the full scale data for the AN/SRN-12 omega antenna, and finally the data on the AT-924B/AN/SRA-17C portside antenna is shown last in Figs. 6.13a through 6.13h.

In addition to the measurements on the antennas, data were also obtained for the currents on the main mast at several points. These points are designated in rable 1.2. Shown here are the scaled up currents and current spectra for the four measurement points. Figures $6.14 \mathrm{a}$ through $6.16 \mathrm{~b}$ show the currents measured on the forward, portside leg of the main mast. Note that these currents are shown for only one of the four mast legs, and that there are also cross brace members which provide alternate circuit flow paths. Figure 6.17 shows the currents measured on one of the hor izontal cross members of the tower at level $\mathbf{G}$.

\subsection{FULL-SCALE INTERNAL COURLING PREDICTIONS}

Details of the locations of the internal bulk current measurement points were given earlier in Figs. 4.3a through $4.3 \mathrm{c}$. In this section we show the result of scaling up the model measurements to the fuii-scale huron level, and compute the current levels for the EMPRESs incident field. 
Figure 6.18a shows the spectrum of the full-scale current measured on the cable leading to the EWER, and Fig. 6.18b shows the equivalent time domain current pulse. (We only measured currents for the internal coupling.) In a similar fashion Figs. 6.19 through 6.23 show the respective currents in the EWCR, TACAN ROOM, TX-1 ROOM, TX-2 ROOm, and the CCR.

\subsection{DISCUSSION OF MEASUREMENTS}

This section points out some of the limitations and uncertainties involved in scale-model measurements and the subsequent data processing involved in extrapolating to the full-scale level.

Our measurement techniques, which have been validated in an earlier work, 3 provide accurate estimates of full-scale coupling to complex EM structures. There are, however, limitations to the accuracy obtainable from a given model. Factors that must be considered, for example, are the model size relative to the working frequency range.

In general, most of the energy coupling occurs in the frequency range around the first resonance of an antenna. This means that the scale model structure must be of a proper size if meaningful measurements are to be obtained. (The UHF antennas on the mast were too small when scaled by the $1 / 48$ factor, therefore, we were not able to make measurements on these antennas.) Model detail can also have a bearing on the results obtained. Mutual effects between the antennas and a large structure such as the main mast can cause significant changes in the measurements.

Details of the internal configurations can also have a large impact on the quality of the irternal EM coupling measurements. As mentioned earlier, we did not accurately model the internal configurations of the ship; cherefore, the internal-coupling measurements should be judged in that light. The data processing techniques can also introduce errors and uncertainties. This is particularly true of the operations that involve the taking of Fourier transforms of data over a finite sample window. Truncation $r^{r}$ data, which occurs due to our limited time-domain sample window, can cause problems if the signal has not sufficiently diminished. We employed a specialized Fourier transform technique (Filon's method ${ }^{4}$ ) for processing our 
data. This technique allows us to use unevenly spaced data in time and zero filling of data records and thus effectively extends the lower frequency range of the transforms. Causality problems $(F(t)=0$ for $t<0$ ) can also occur when inverse transforms are taken of non-causal data. There is some eridence that we may have had problens with this in some of the measurements and extrapolations. 


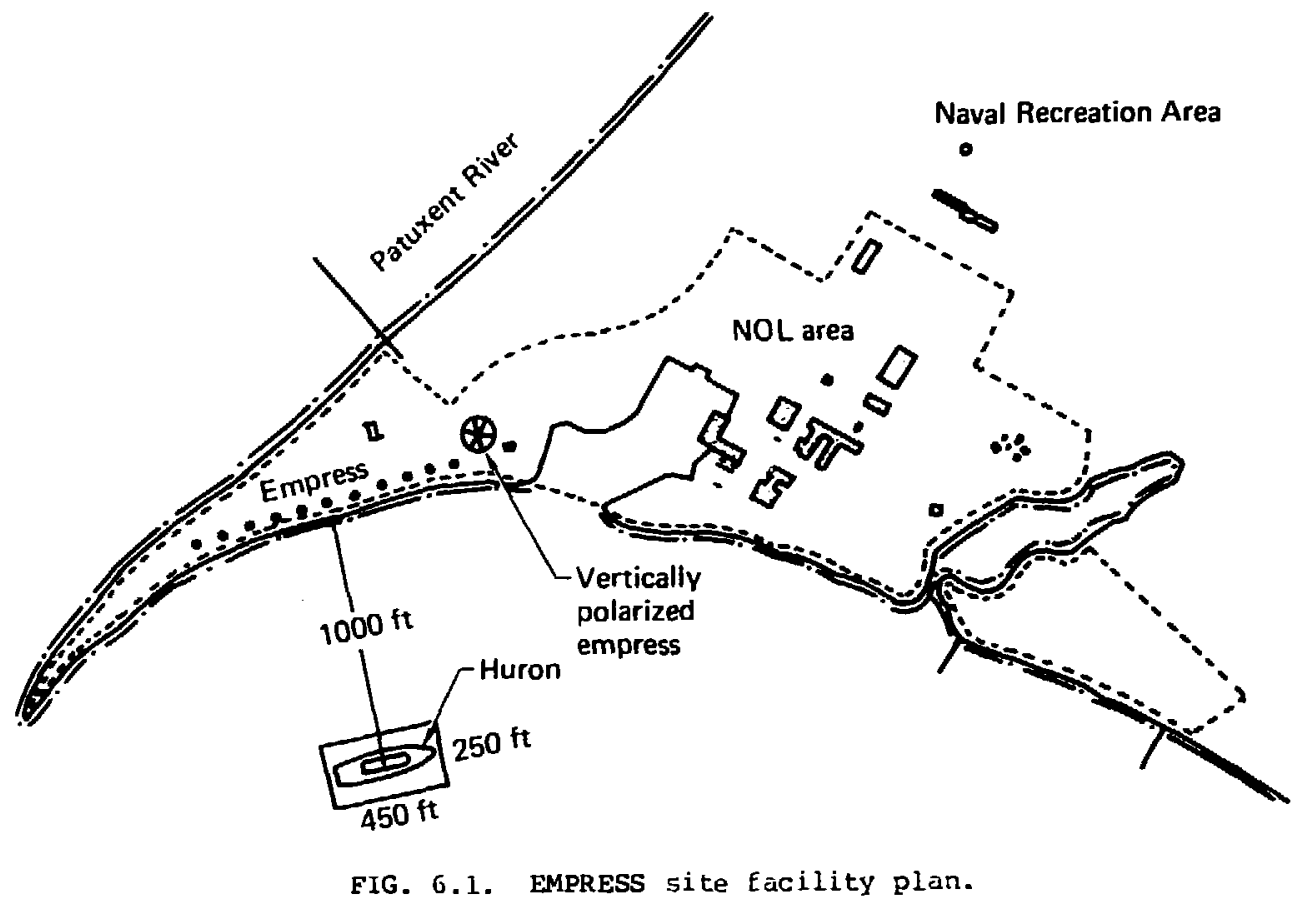




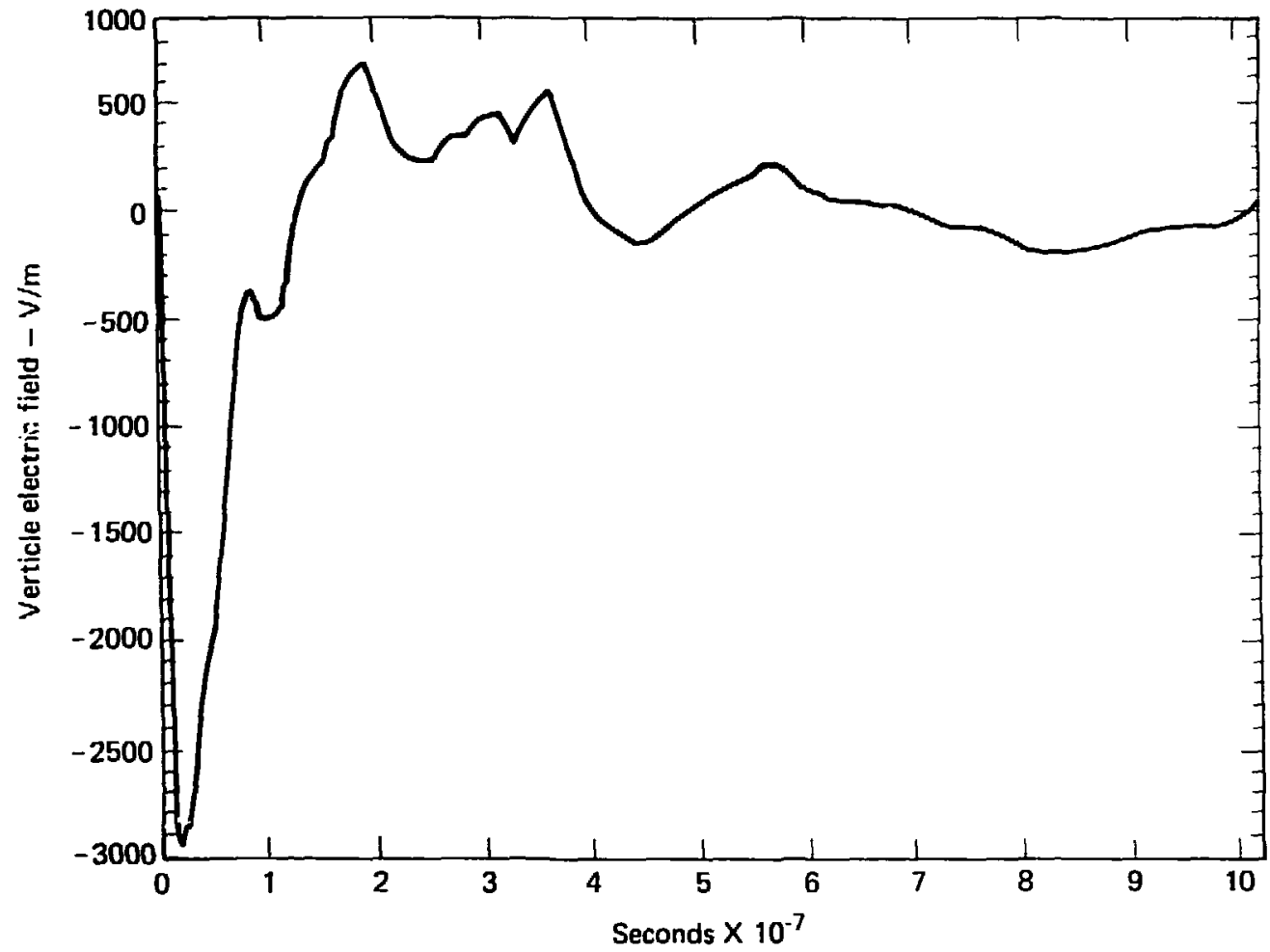

FIG. 6.2. Vertical electric field produced by EMPRESS simulator (field measured at ship anchor point). 


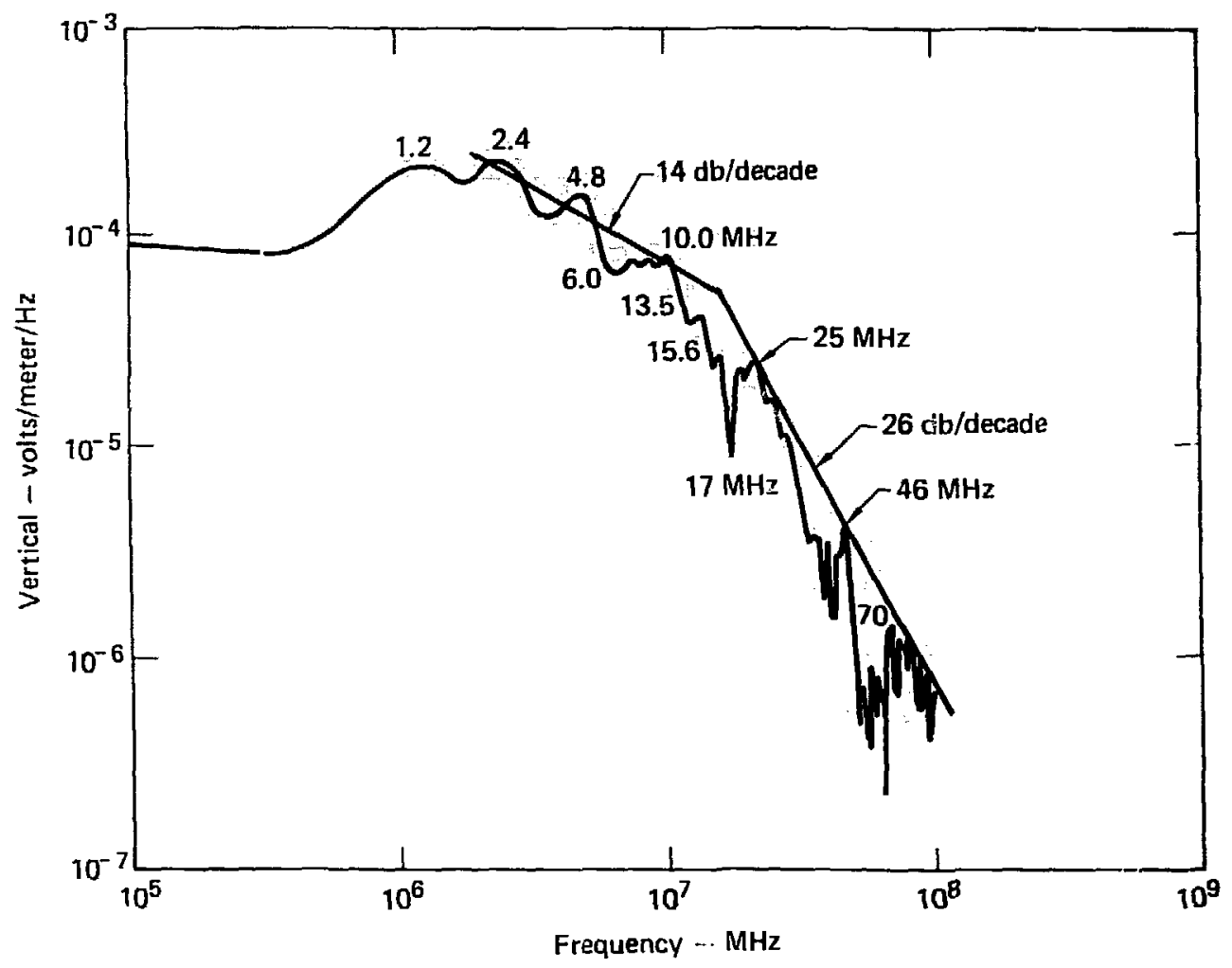

FIG. 5.3. EMPRESS vertical electric Eield at ship anchor point. 


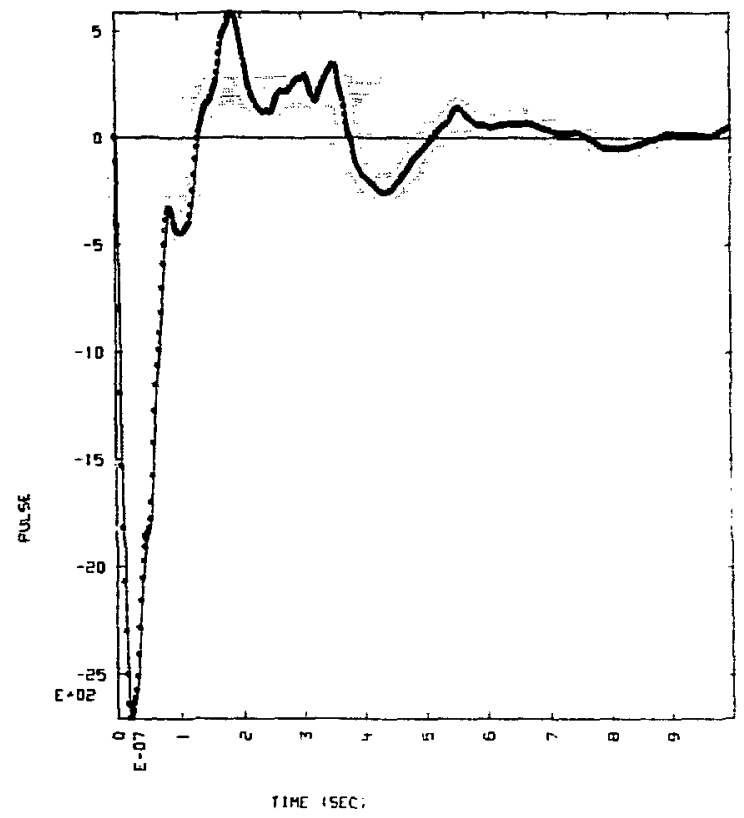

FIG. 6.4. LLL digitized EMPRESS puise.

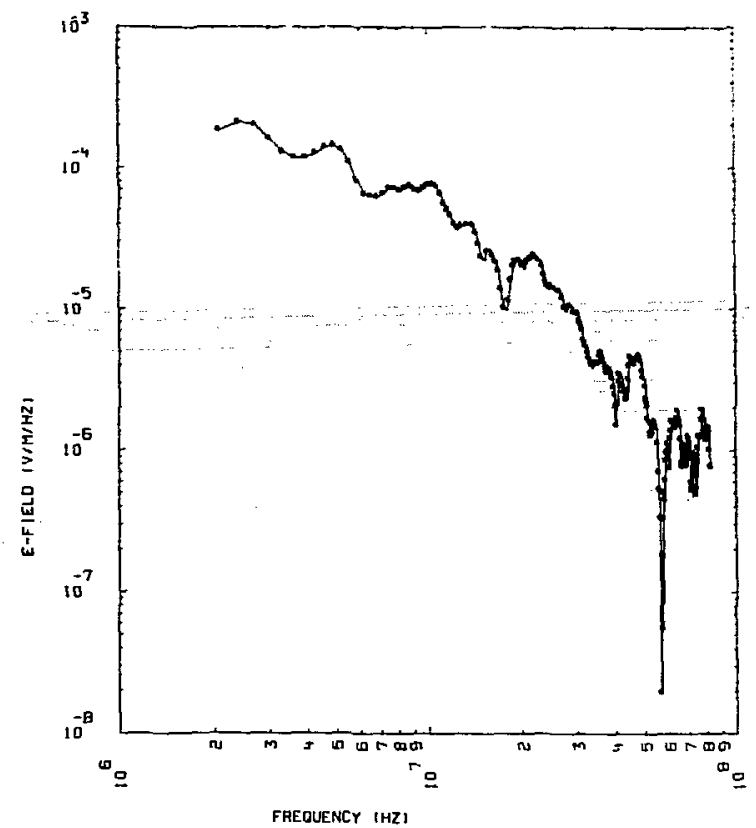

FIG. 6.5. Spectrum of EMPRESS pulse derived from digitized pulse waveform. 6-9 


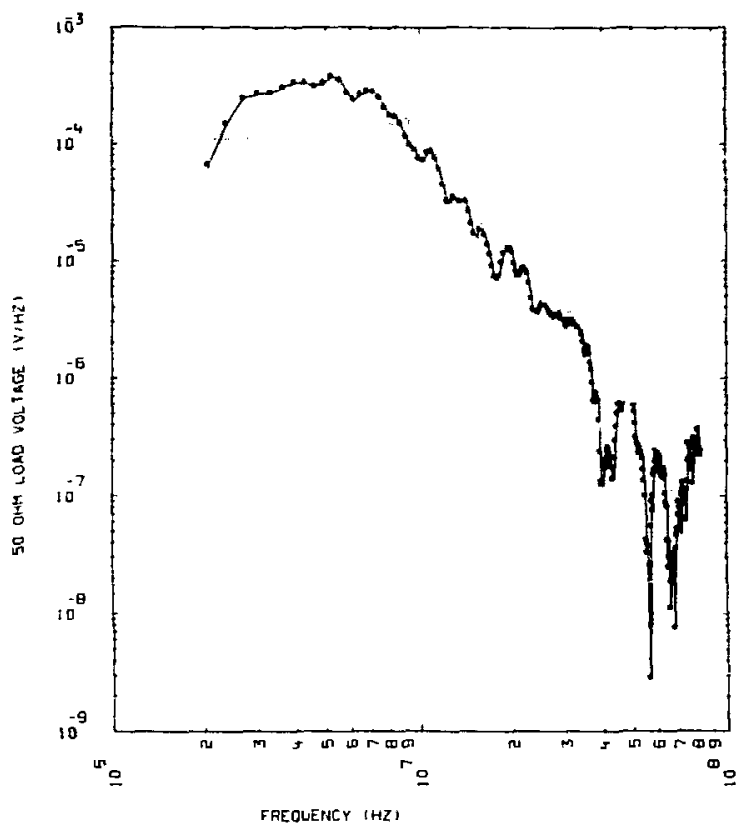

FIG. 6.6a. Predicted full-scale frequency response spectrum - MF/HF transmit 35-ft whip (port).

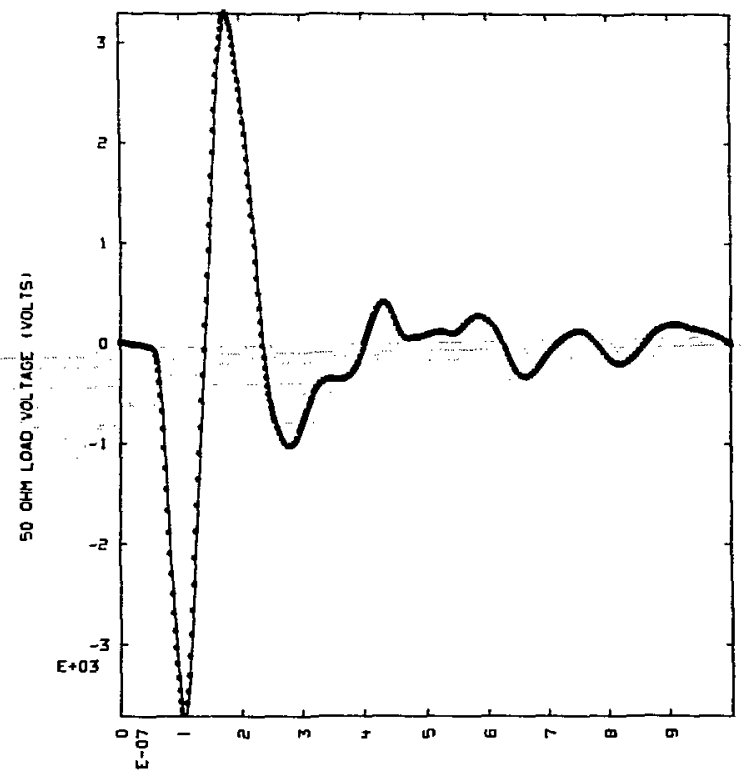

TIME ISEC:

FIG. 6.6b. Predicted full-scale transient response - MF/HF transmit 35-ft whip (port). 


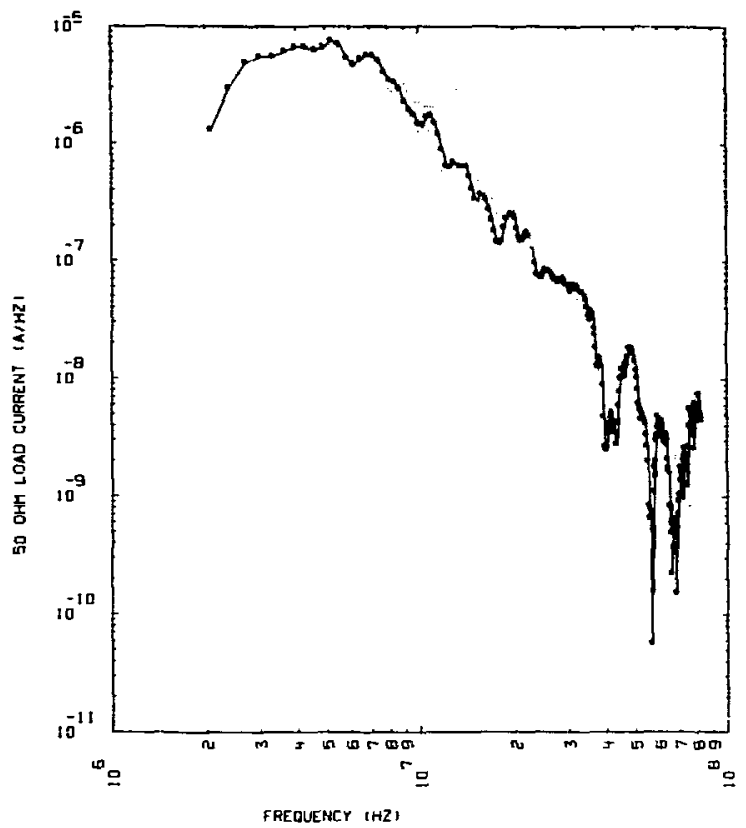

FIG. 6.6c. Predicted full-scale frequency response spectrum - MF/HF transmit 35-ft whip (port).

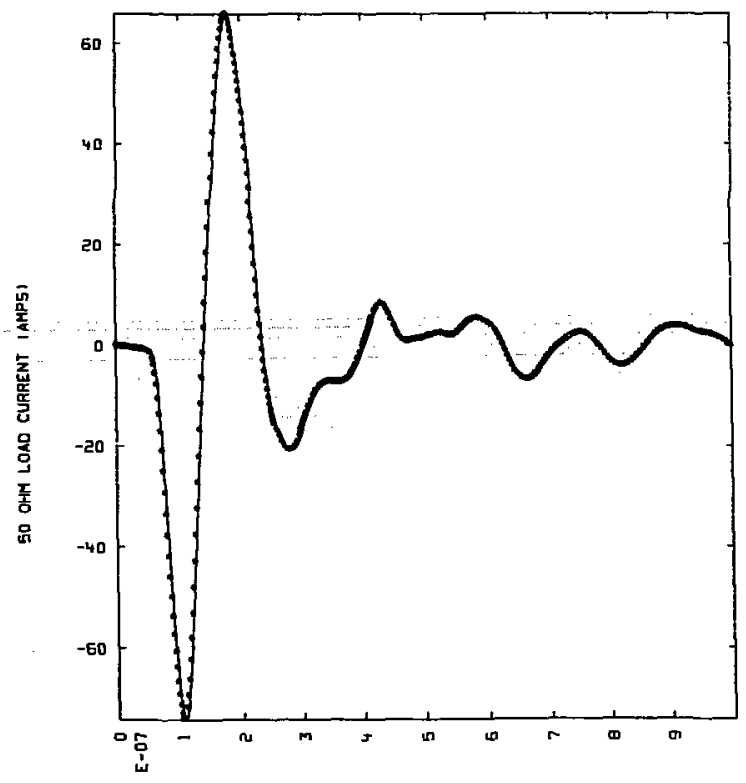

TIHE ISEC:

FIG. 6.6d. Predicted full-scale transient response - MF/HF transmit 35-ft whip (port). 


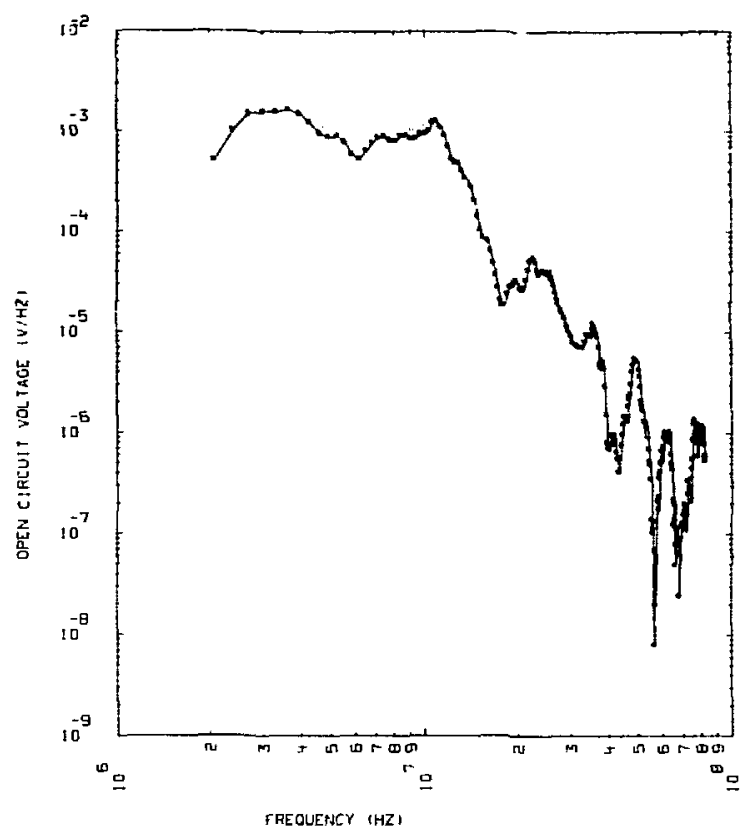

FIG. б.6e. Predicted full-scale Erequency response spectrum - MF/HF transmit 35-Et whip (port).

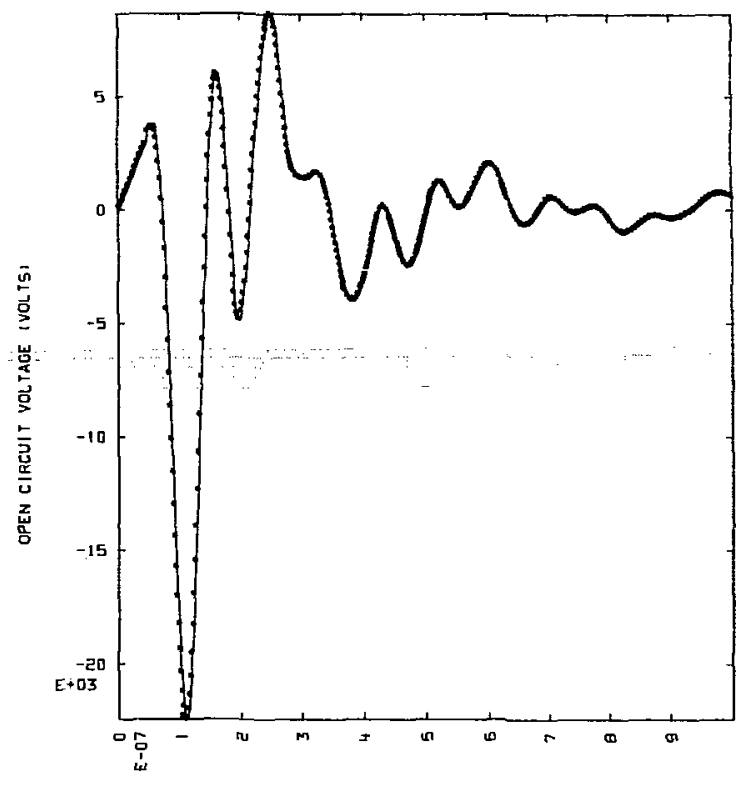

TIHE ISEC.

FIG. 6.6f. Predicted full-scale transient response - MF/HF transmit 35-ft whip (port). 


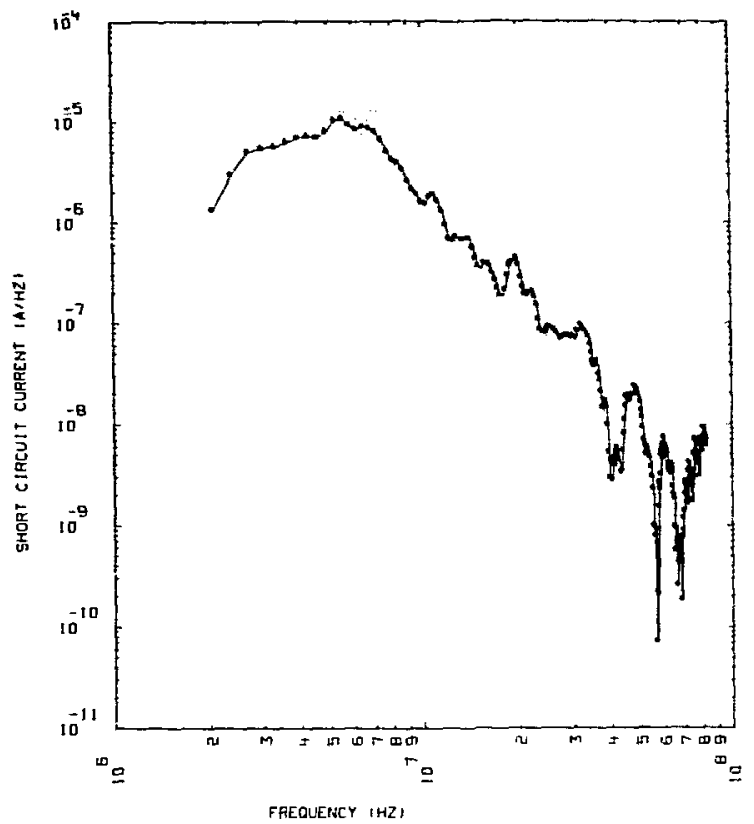

FIG. 6.6g. Predicted full-scale frequency response spectrum - MF/HF transmit 35-ft whip (port).

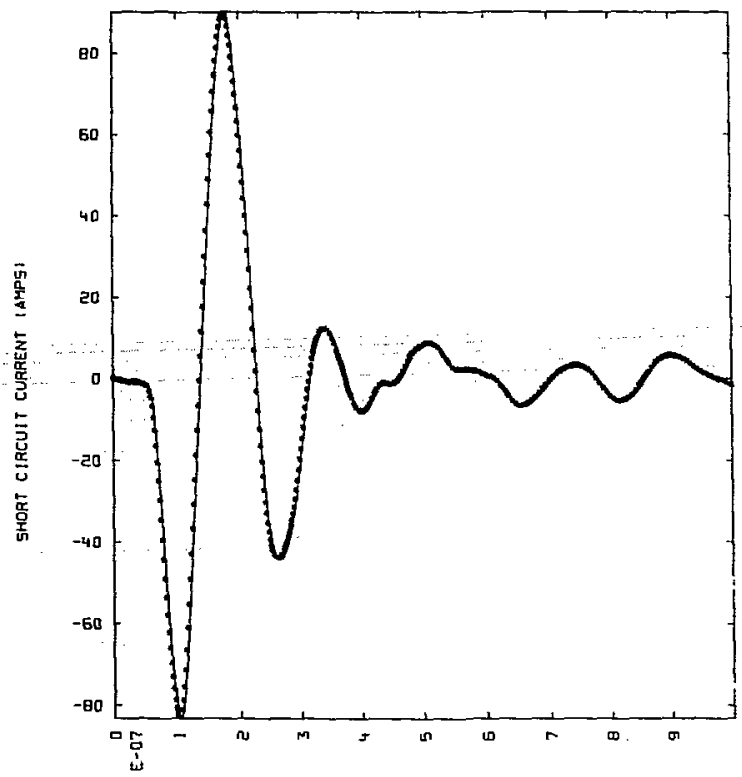

TIHE ISEC:

FIG. 6.6h. Predicted full-scale transient response - MF/HF transmit 35-Ft whip (port). 


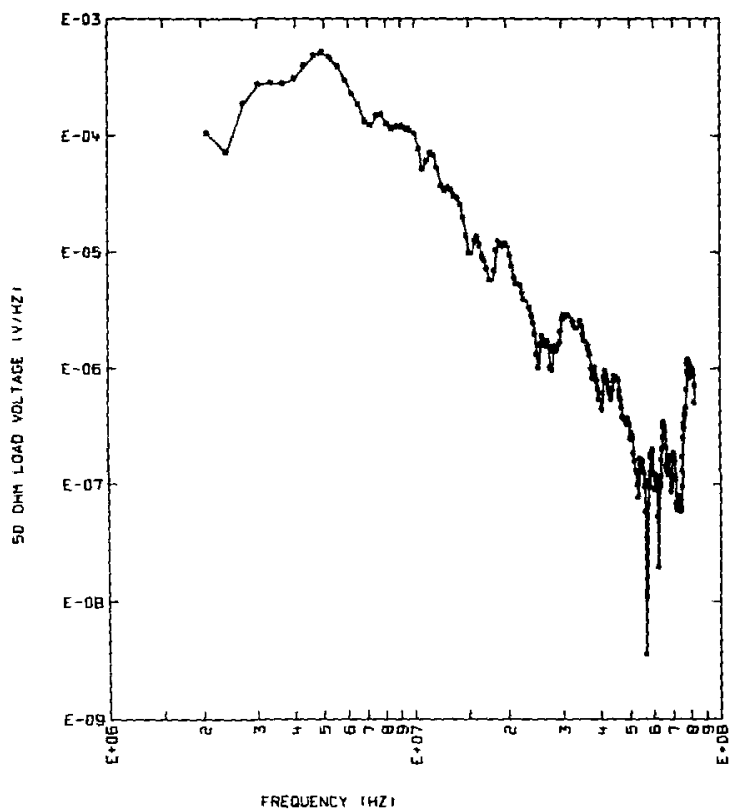

EIG. 6.7a. Predicted full-scale frequency response spectrum - MF/HF transmit 35-ft whip (starboard).

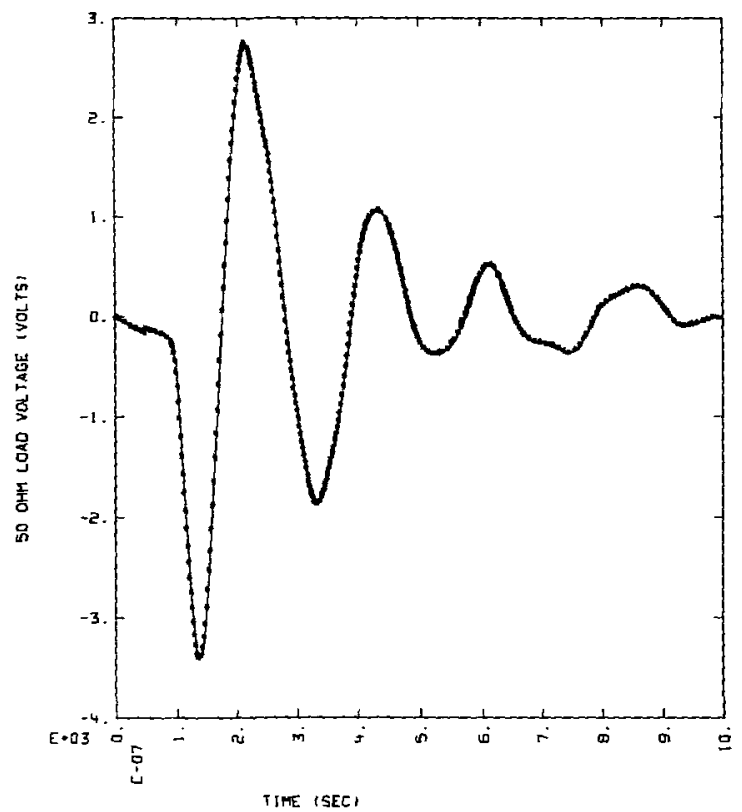

EIG. 6.7b. Predicted full-scale transient response - MF/HF transmit 35-Et whip (starboard). 


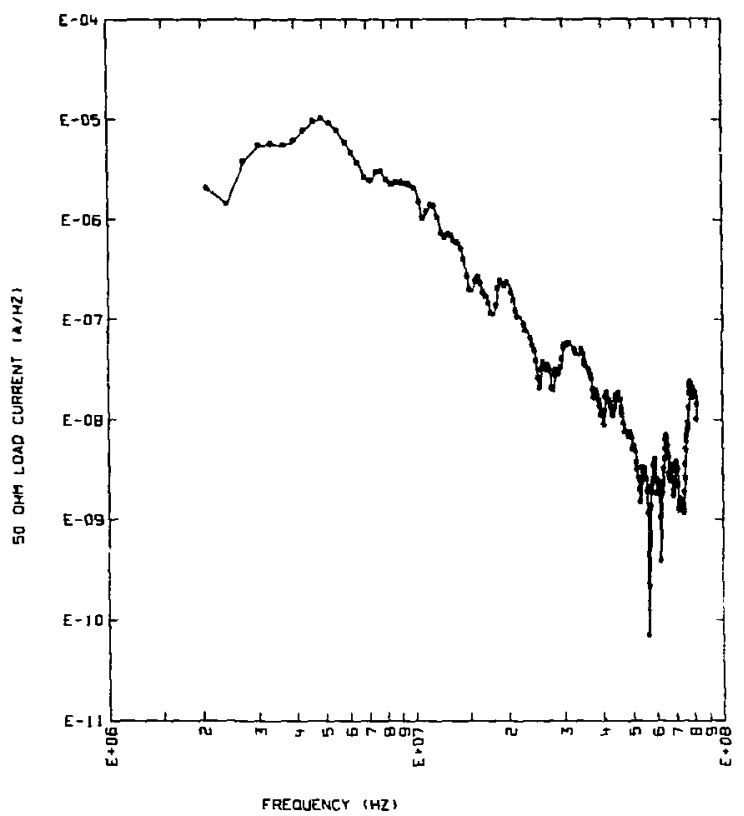

FIG. 6.7c. Predictec full-scale frequency response spectrum - MF/HF transmit 35-ft whip (starboard).

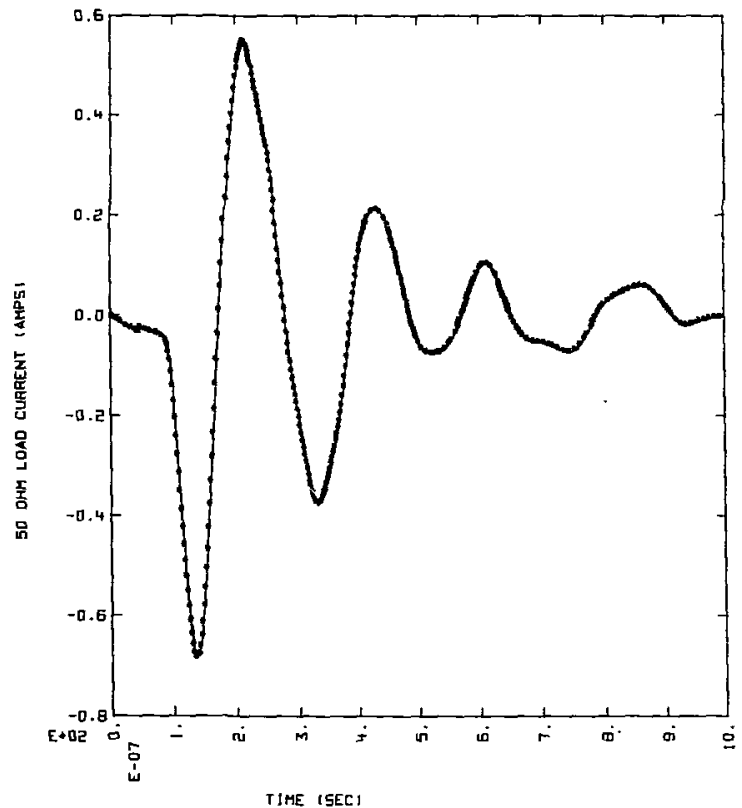

FIG. 6.7d. Predicted full-scale transient response - MF/HF transmit 35-ft whip (starboard). 


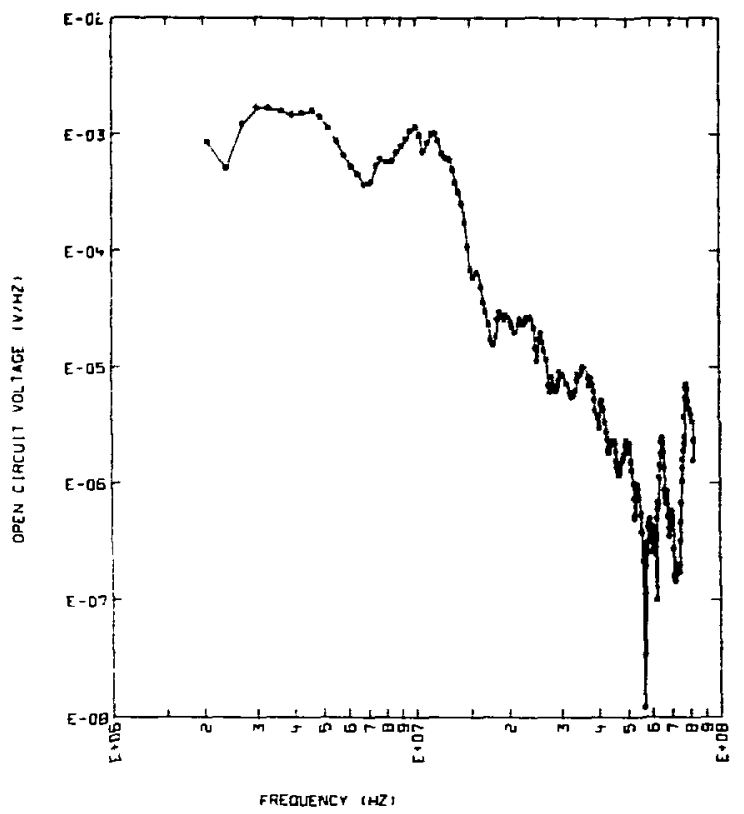

FIG. 6.7e. Predicted full-scale frequency response spectrum - MF/HF transmit 35-ft whip (starboard).

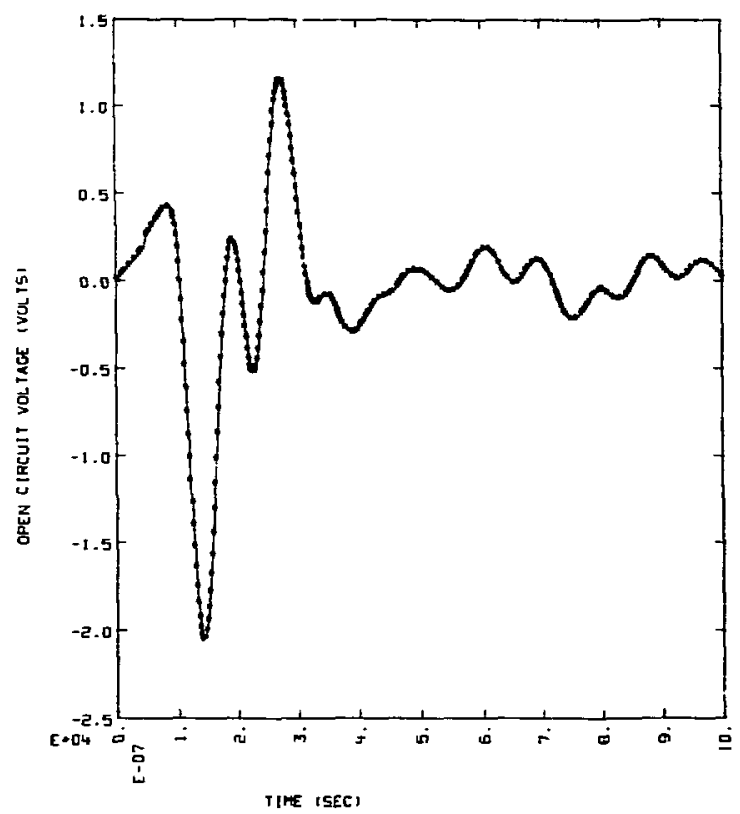

FIG. 6.7f. Predicted full-scale transient response - MF/HF transmit 35-ft whip (starboard). 


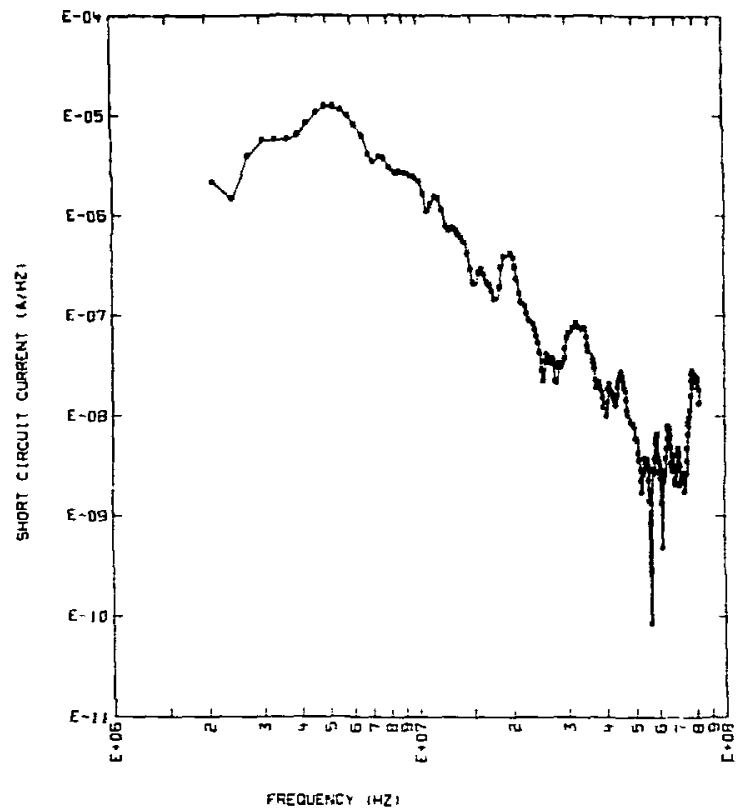

FIG. 6.7g. Predicted íull-scale frequency response spectrum - MF/HF transmit 35-ft whip (starboard).

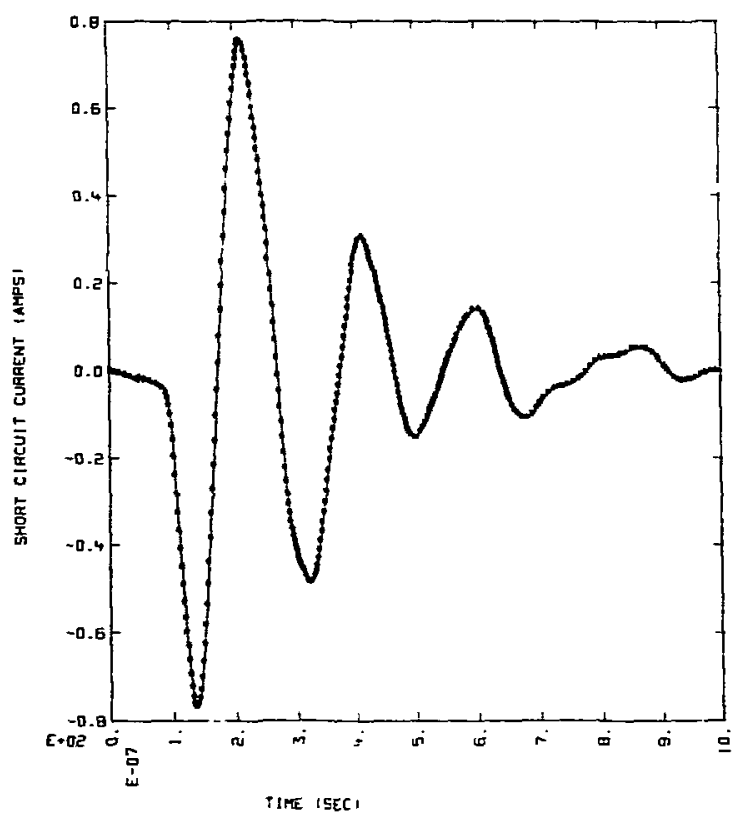

FIG. 6.7h. Predicted full-scale transient response - MF/HF transmit 35-ft whip (starboard). 


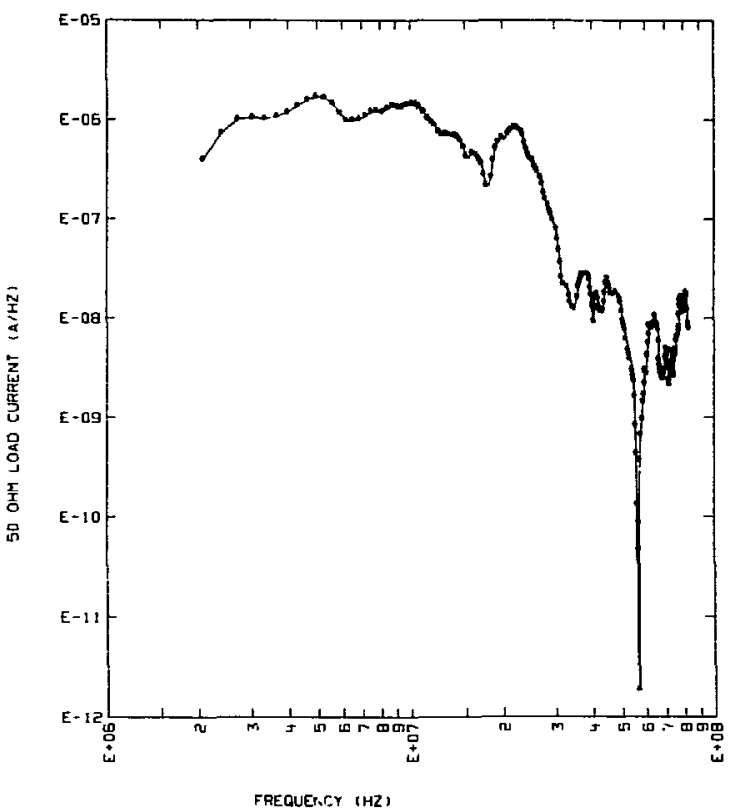

FIG. 6.8a. Predicted full-scale frequency response spectrun - HF receive fan (port).

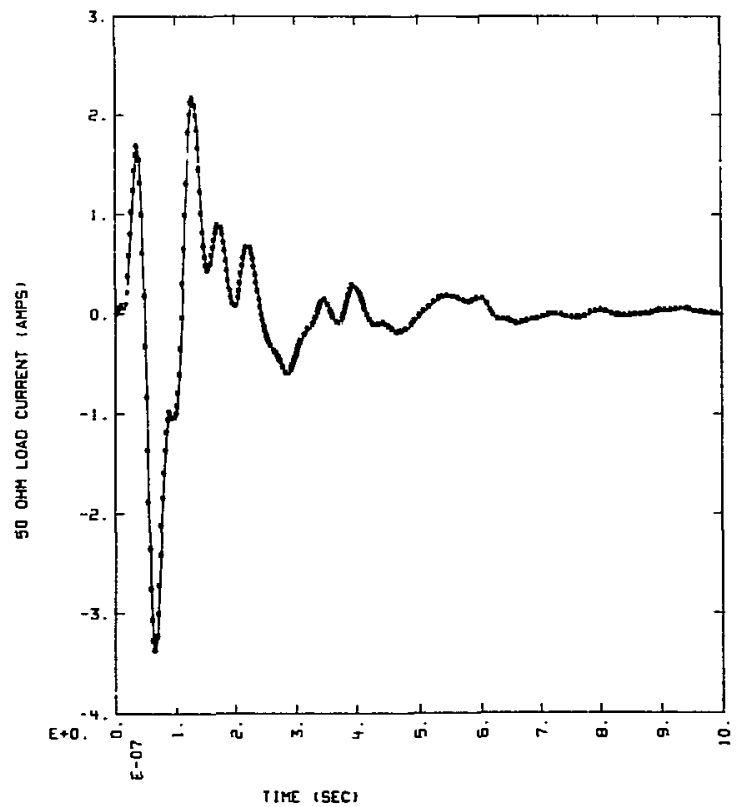

FIG. 6.8b. Predicted full-scale transient response - HF receive fan (port). 6-18 


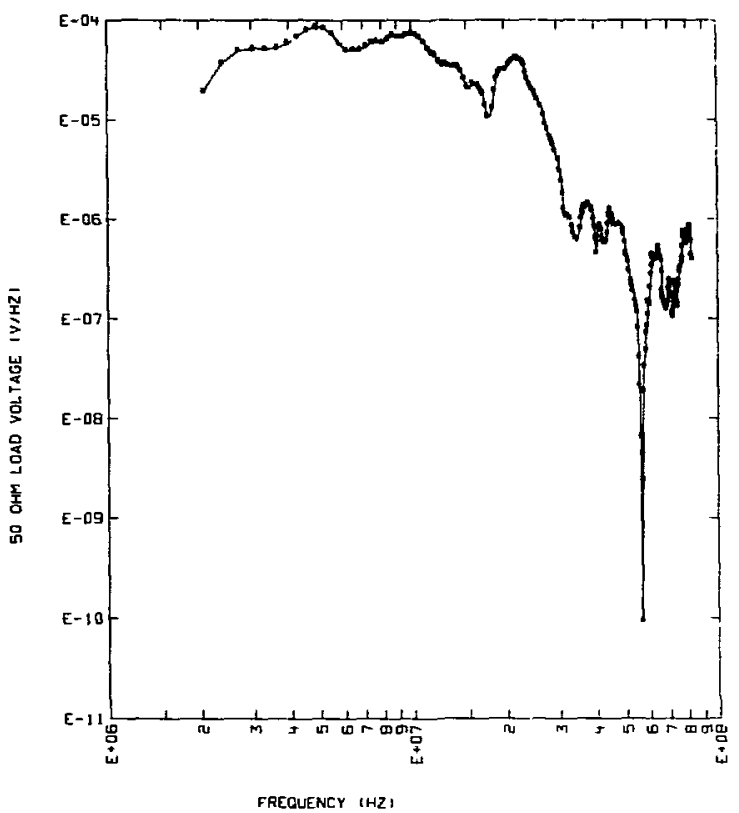

FIG. 6.8c. Predicted full-scale frequency response spectrum - HF receive Ean (port).

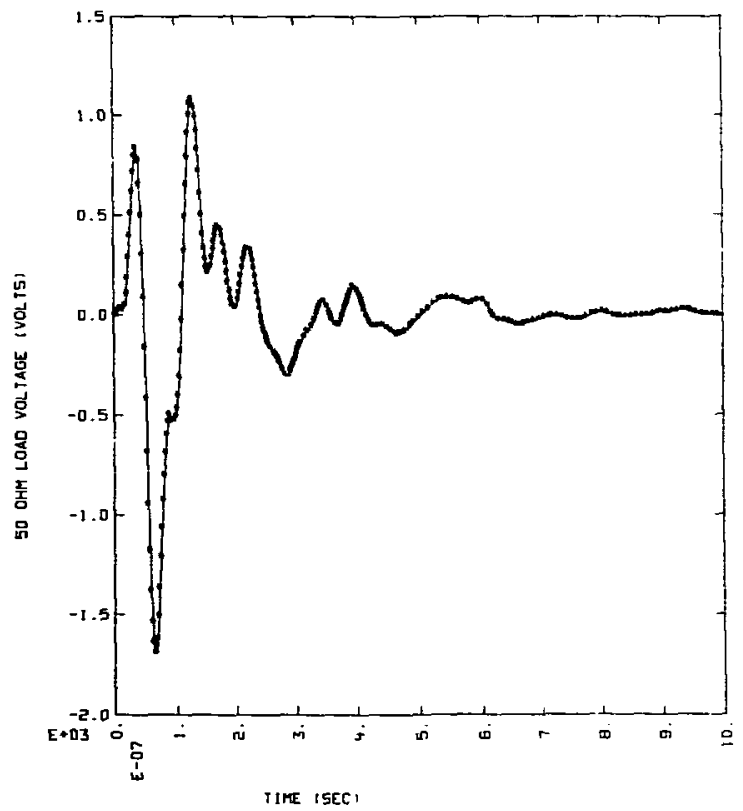

FIG. 6.8d. Predicted full-scale transient response - BF receive fan (port). 


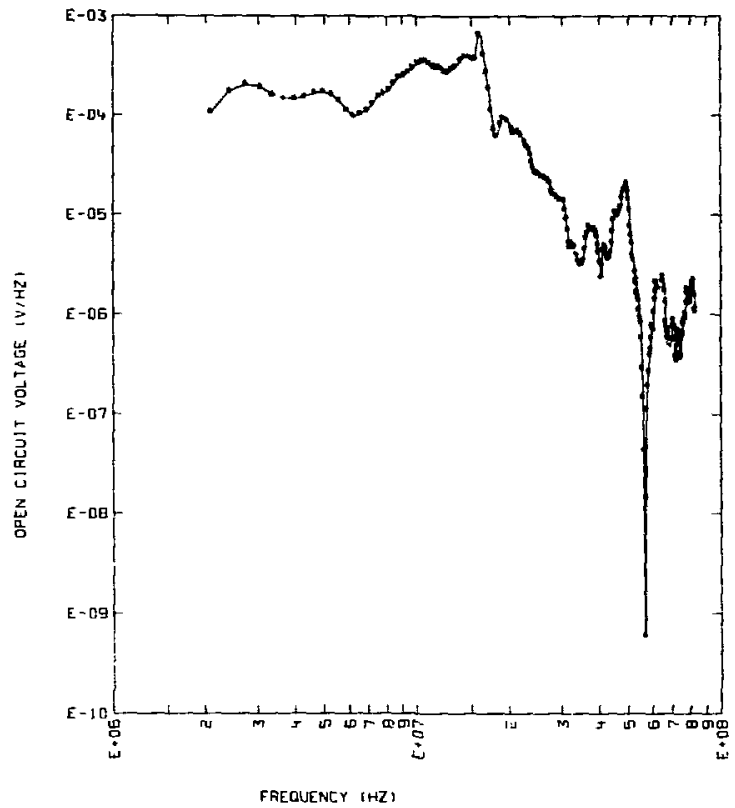

FIG. 6.8e. Predicted full-scale frequency response spectrum - HF receive fan (port).

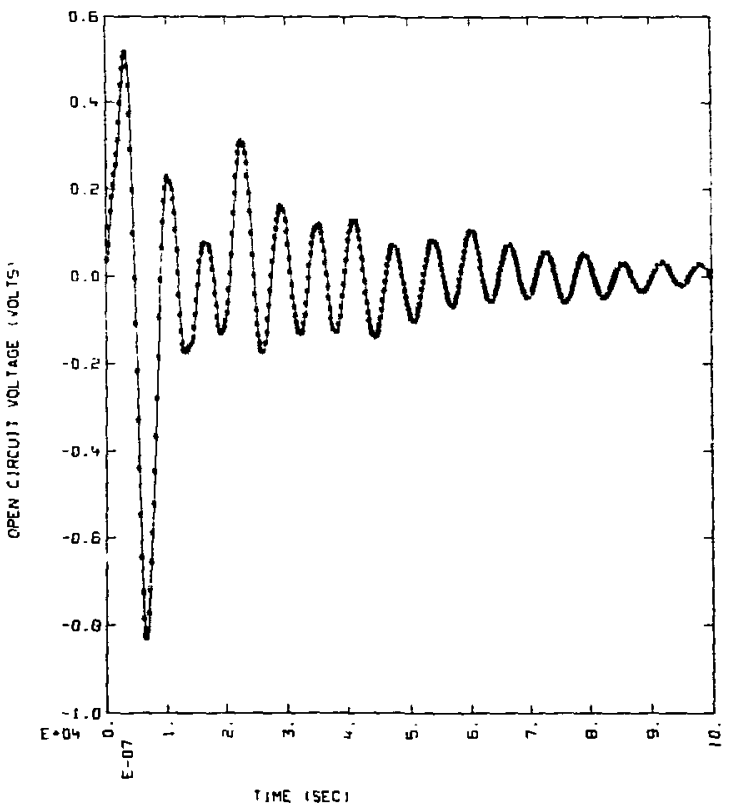

FIG. 6.8f. Predicted full-scale transient response - HF receive fan (port). 


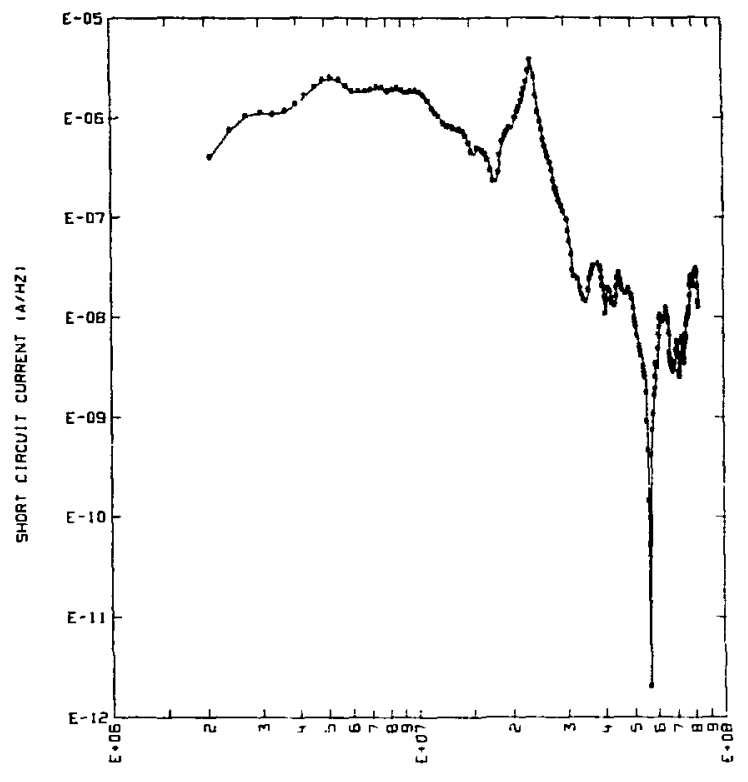

FREOUEACY IHZ

FIG. 6.8g. Predicted full-scale frequency response spectrum - HF receive fan (port).

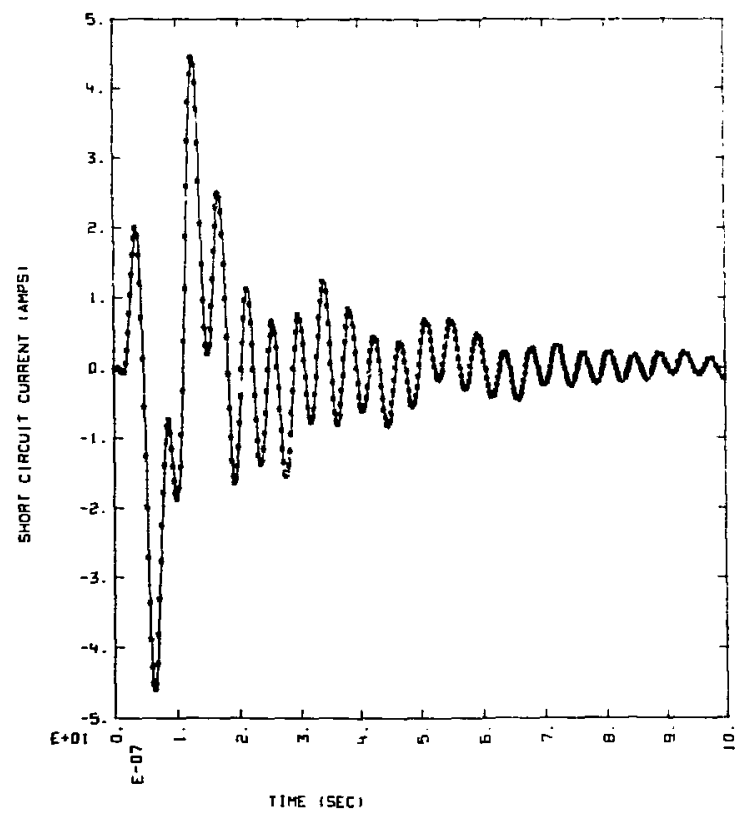

FIG. 6.8h. Predicted full-scale transient response - HF receive fan (port). 


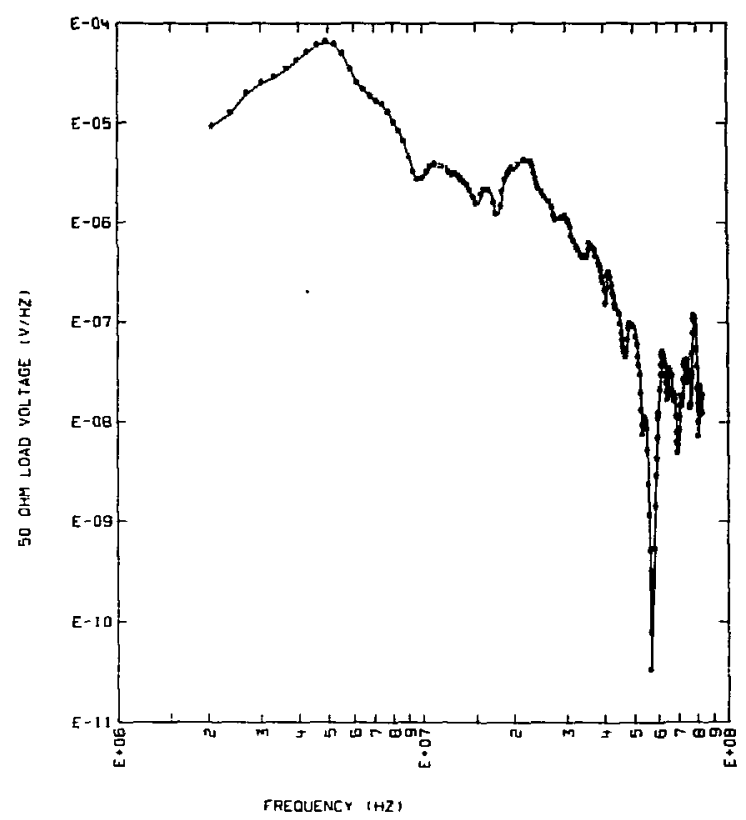

FIG. 6.9a. Predicted full-scale frequency response spectrum - HF receive fan (starboard).

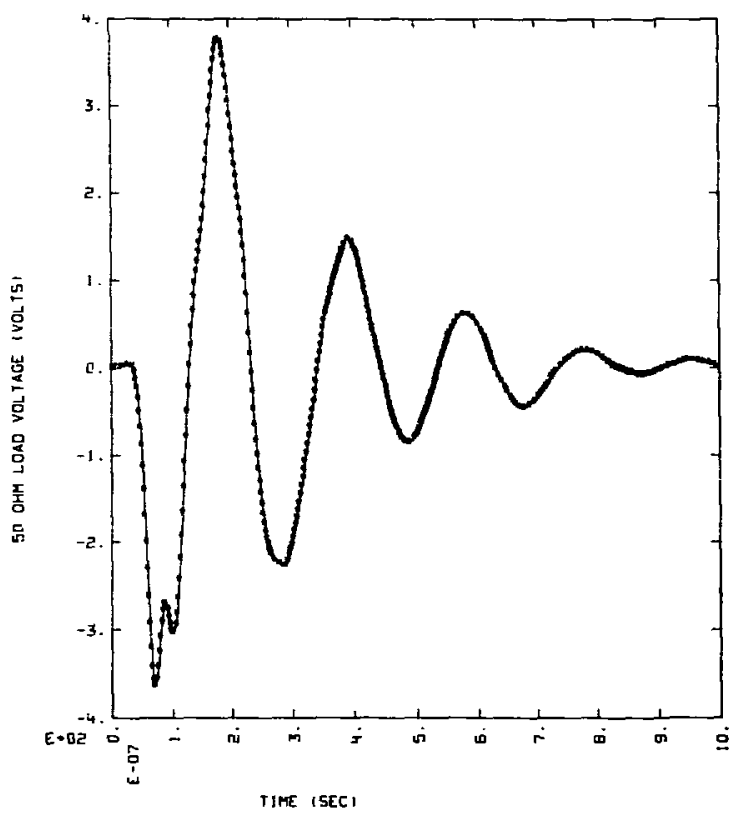

FIG. 6.9b. Predicted full-scale transient response - HF receive fan (starboard). 


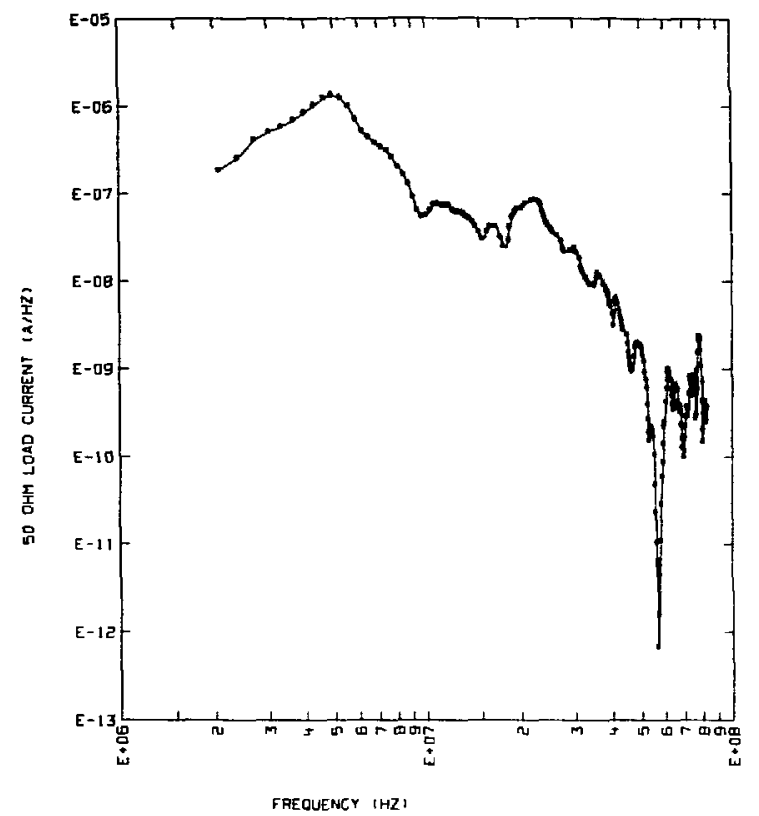

FIG. 6.9c. Predicted full-scale frequency response spectrum - HF receive fan (starboard).

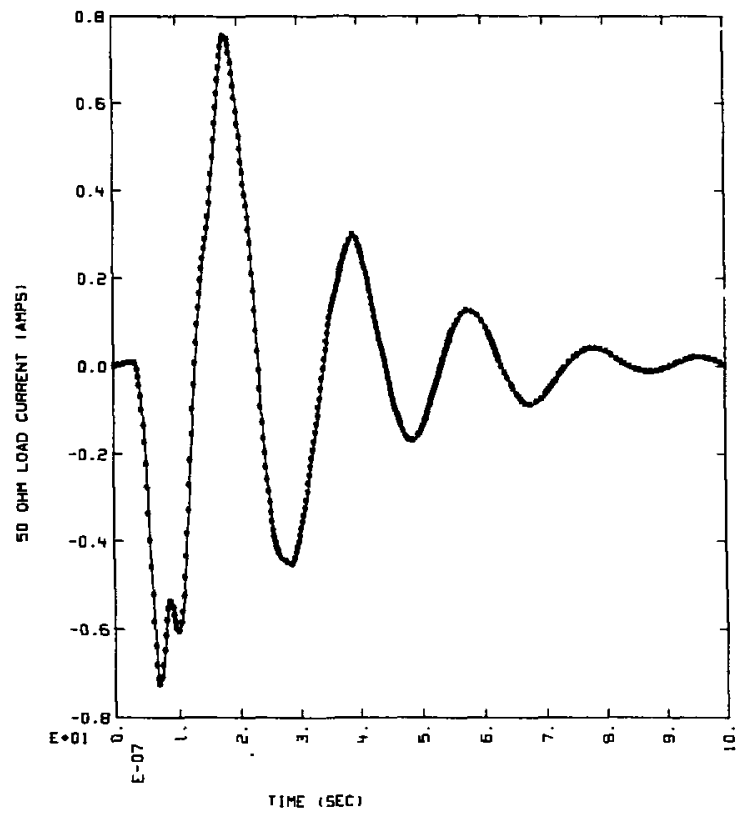

FIG. 6.9d. Predicted full-scale transient response - BF receive Ean (star board) . 


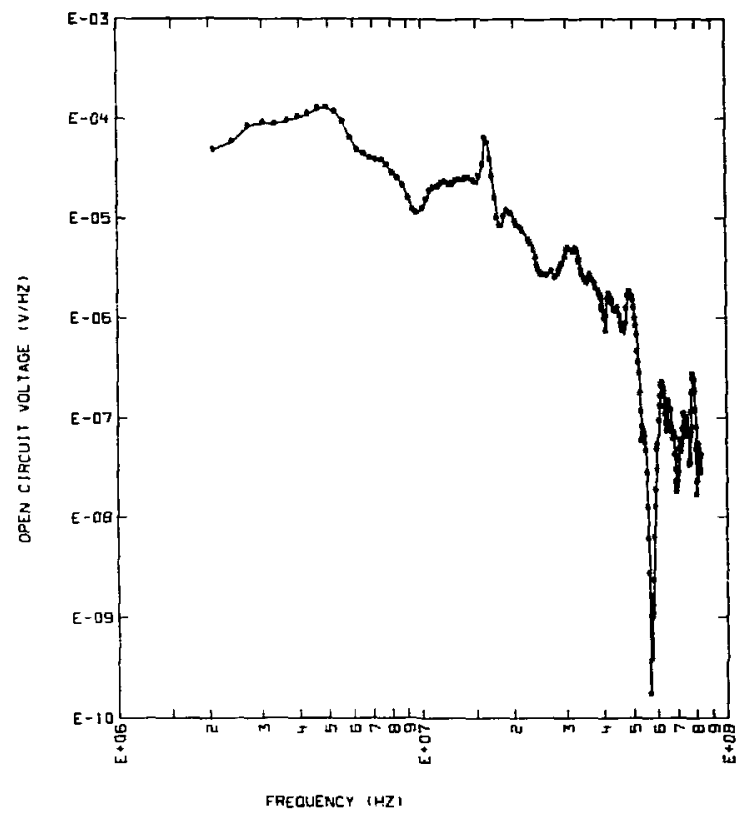

FIG. 6.9e. Predicted full-scale frequency response spectrum - HF receive fan (starboara).

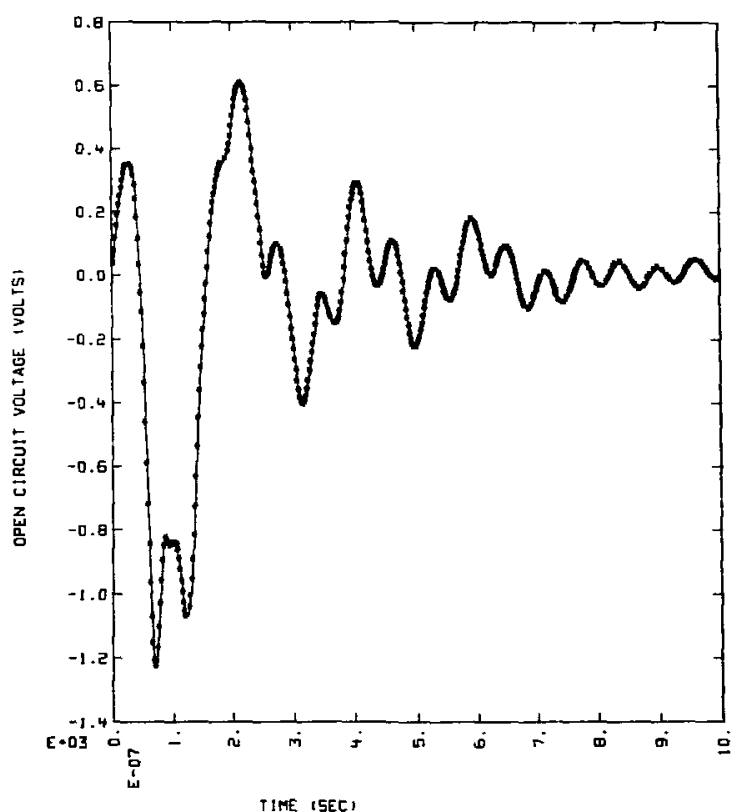

FIG. 6.9f. Predicted full-scale transient response - HF receive fan (starboard). 


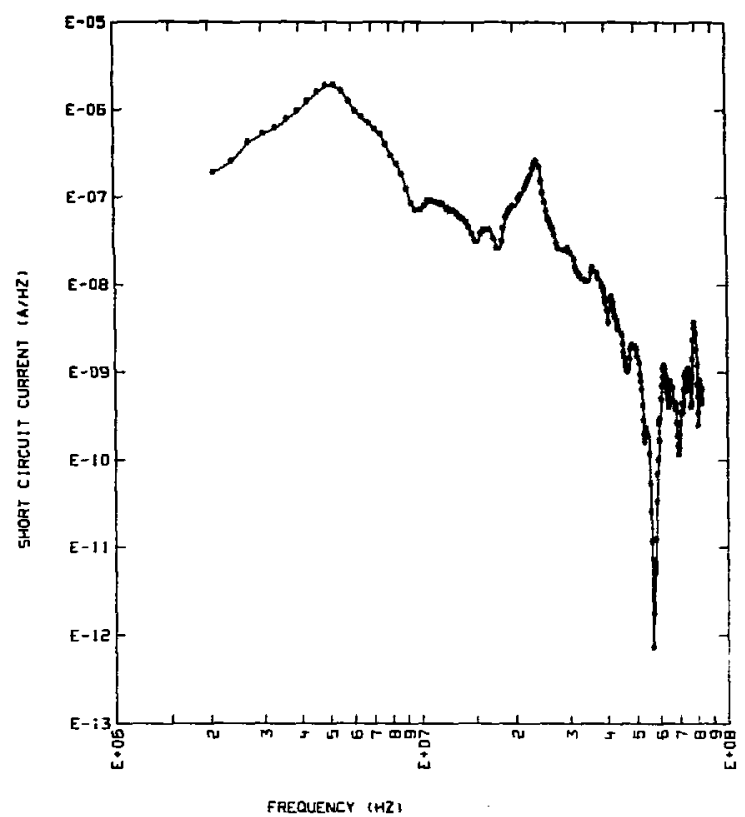

FIG. 6.9g. Predicted full-scale frequency response spectrum - HF receive fan (starboaró).

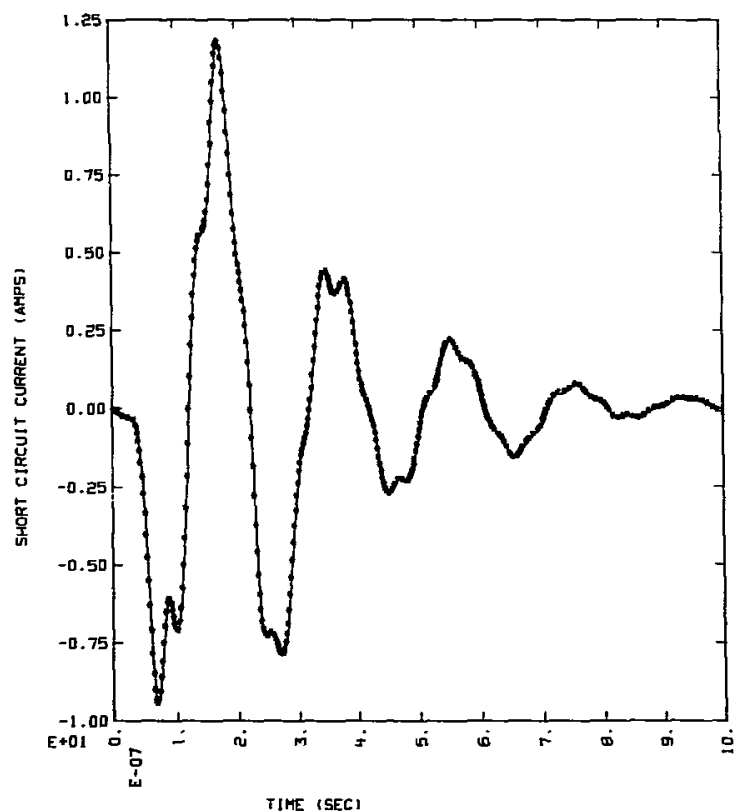

FIG. 6.9h. Predicted full-scale transient response - HF receive fan (starboard). 


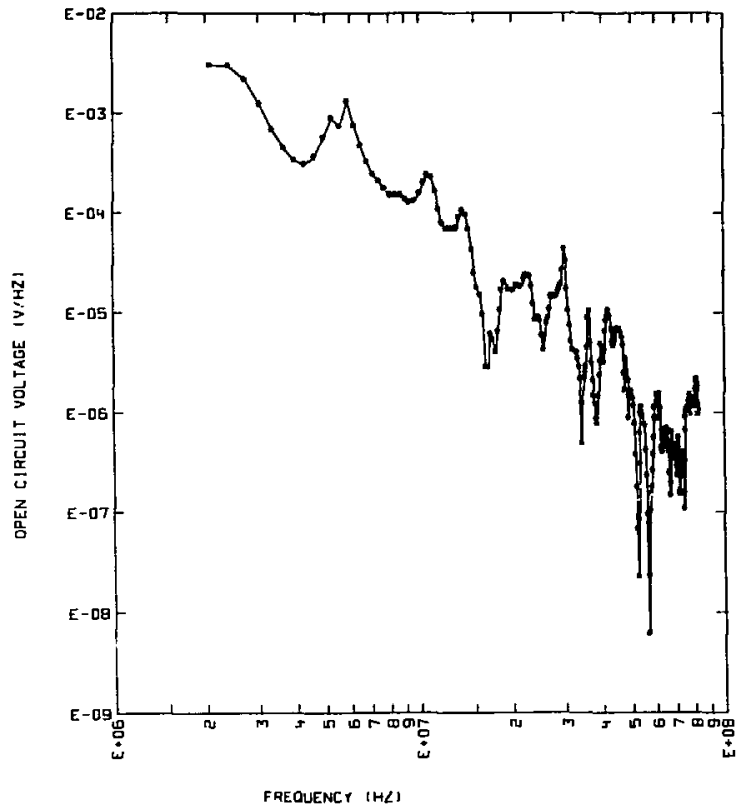

FIG. 6.10a. Predicted full-scale frequency response spectrum - AN/SRC-23 flat top (port).

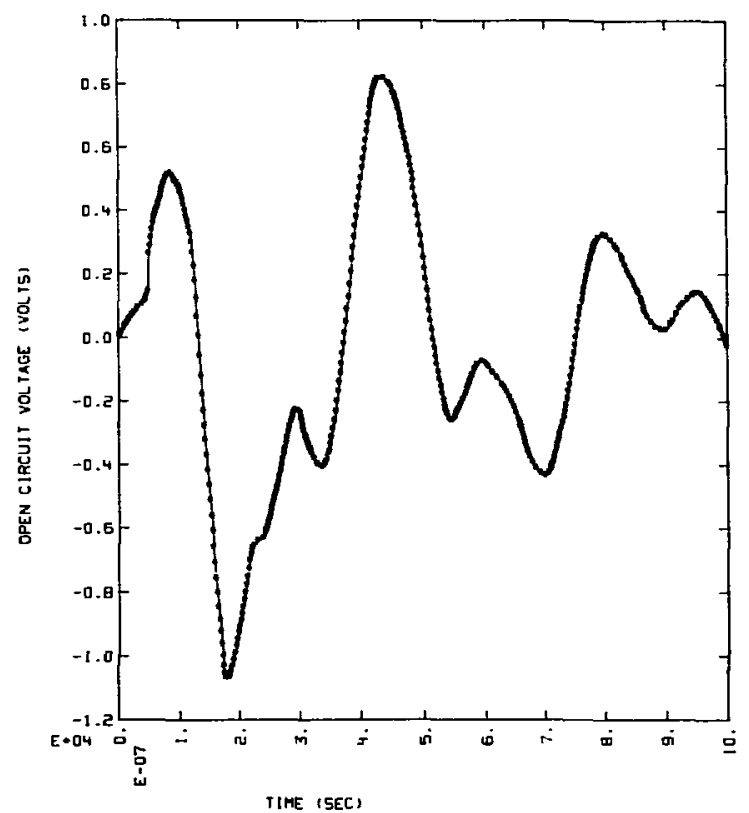

FIG. 6.10b. Predicted full-scale transi’nt response - AN/SRC-23 flat top (port). 


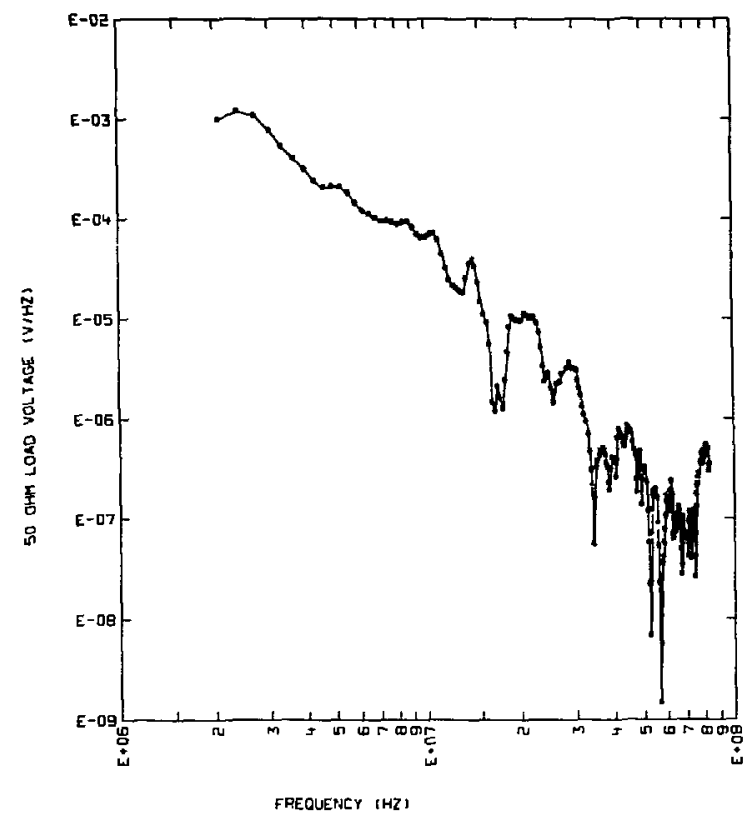

FIG. 6.10c. Predicted full-scale frequency response spectrum - AN/SRC-23 flat top (port) .

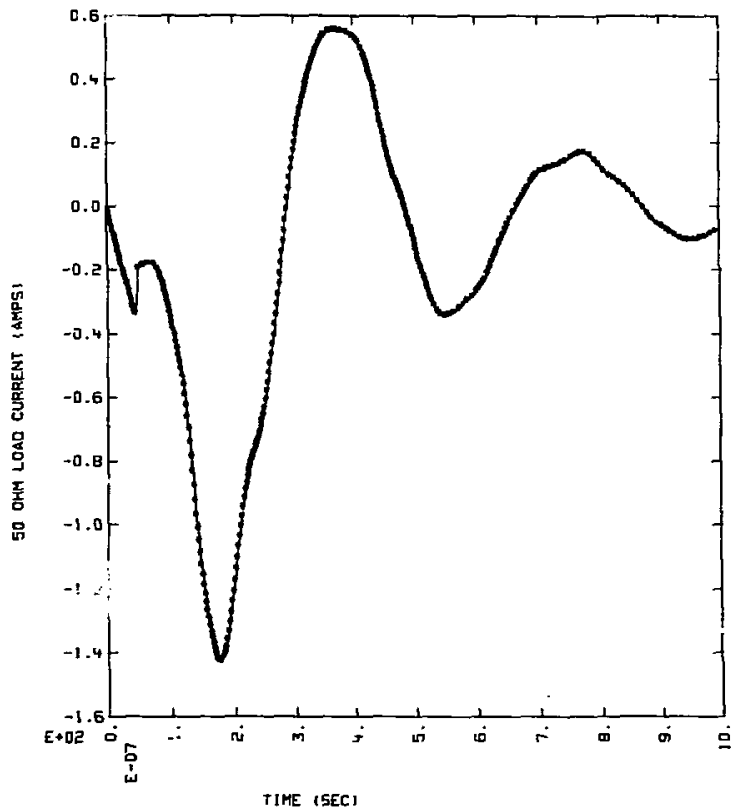

FIG. 6.10d. Predicted full-scale transient response - AN/SRC-23 flat top (port) . 


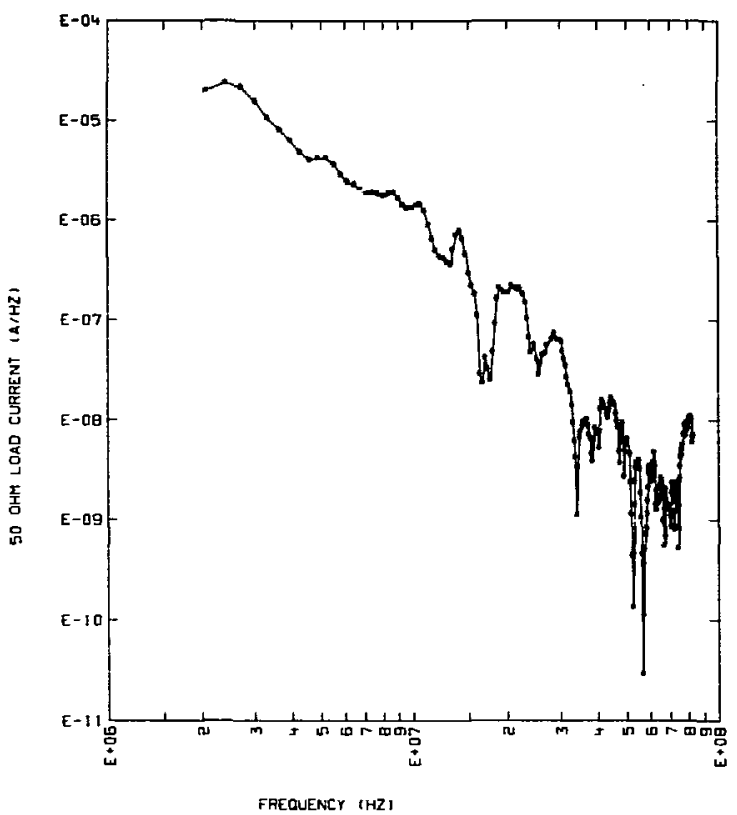

FIG. 6.10e. Predicted full-scale frequency response spectrum - AN/SRC-23 flat top (port).

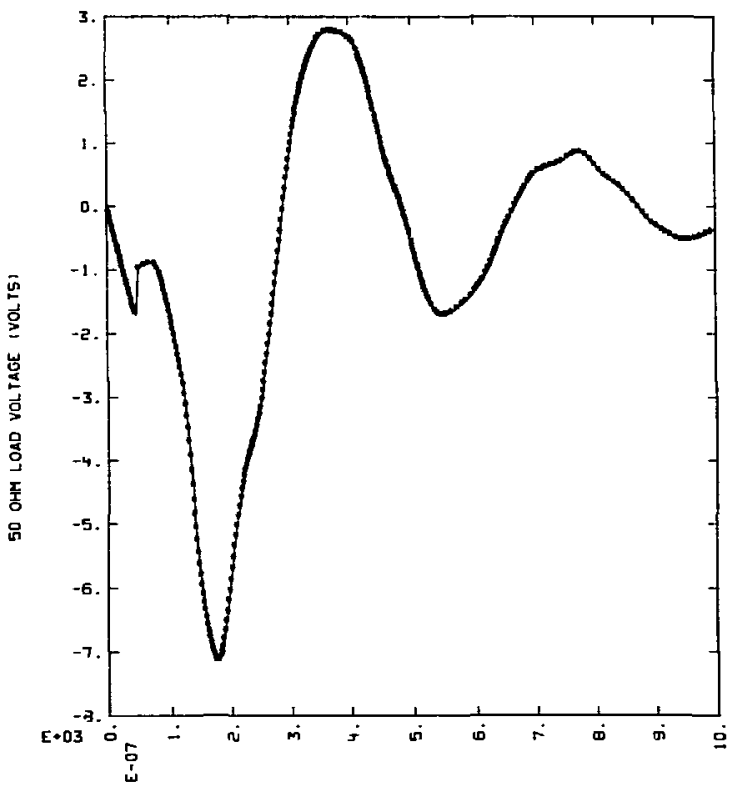

TIME ISEC)

FIG. 6.10f. Predicted full-scale transient response - AN/SRC-23 flat top (port). 


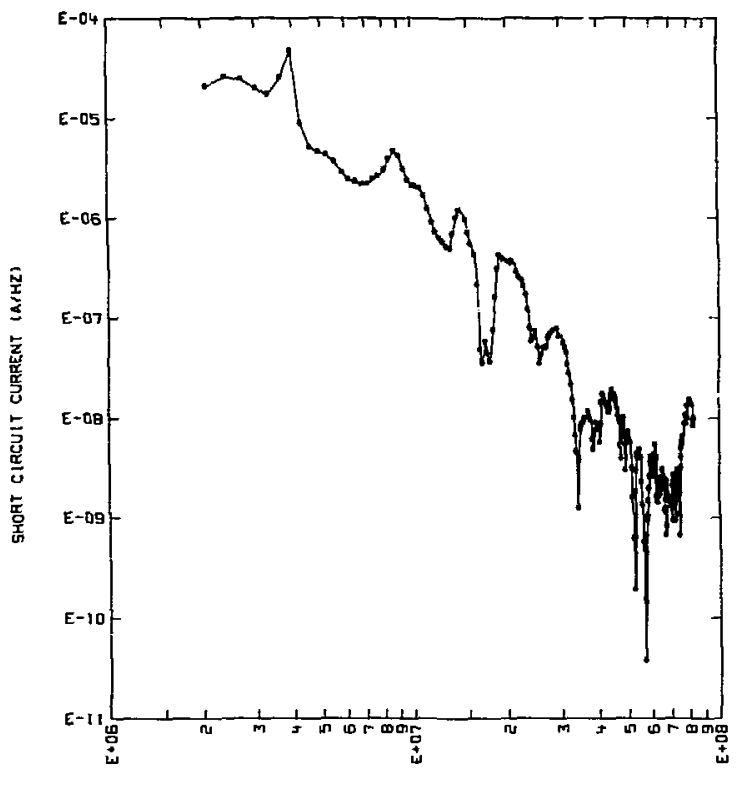

FREOUENCY IHZ)

FIG. 6.10g. Predicted full-scale frequency response spectrum - AN/SRC-23 flat top (port).

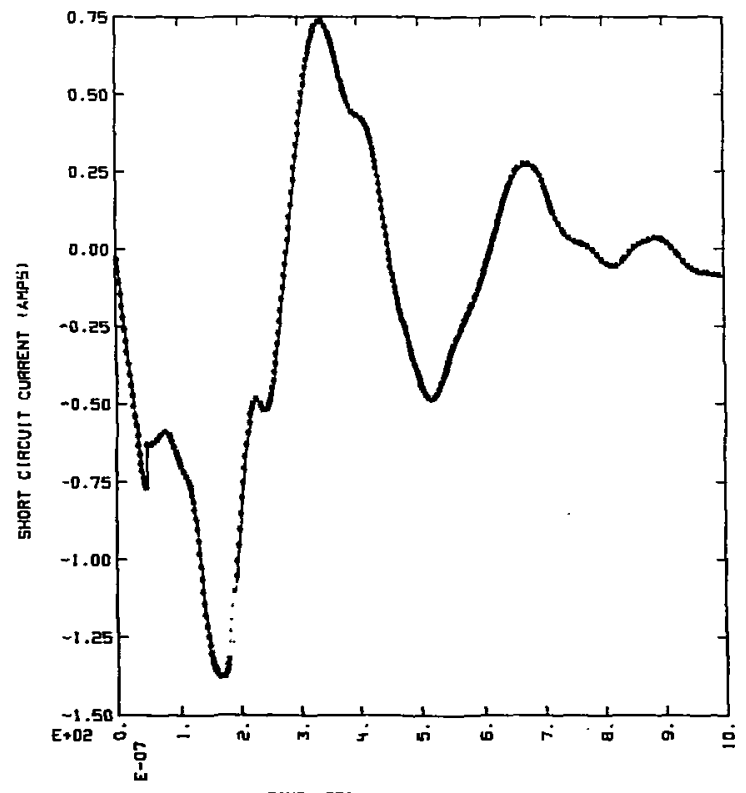

JIHE (SSEC)

FIG. 6.10h. Predicted full-scale transient response - AN/SRC-23 flat top (port). 


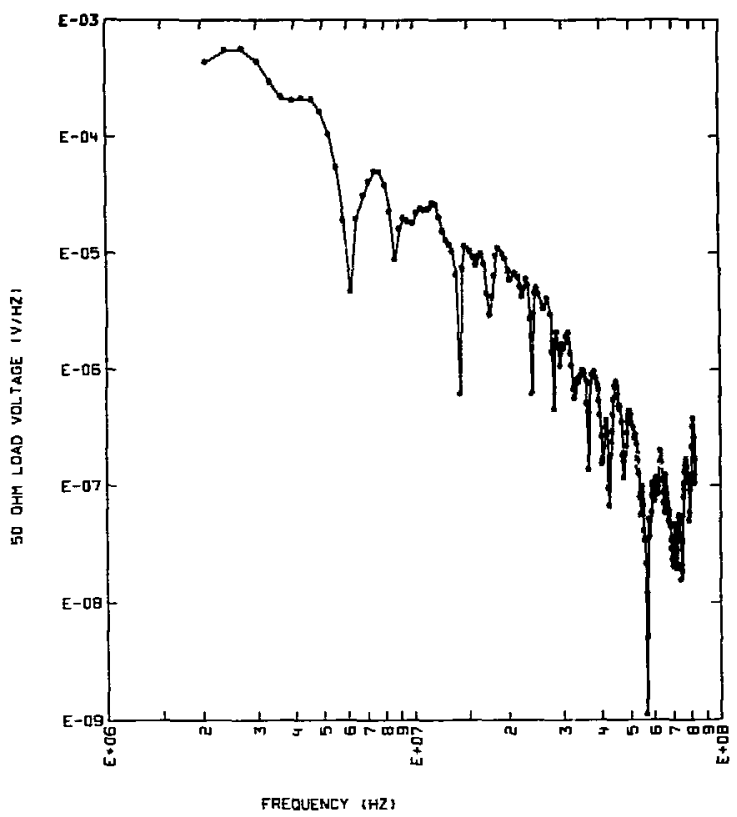

FIG. 6.1la. Predicted full-scale frequency response spectrum - AN/SRC-23 flat top (starboard).

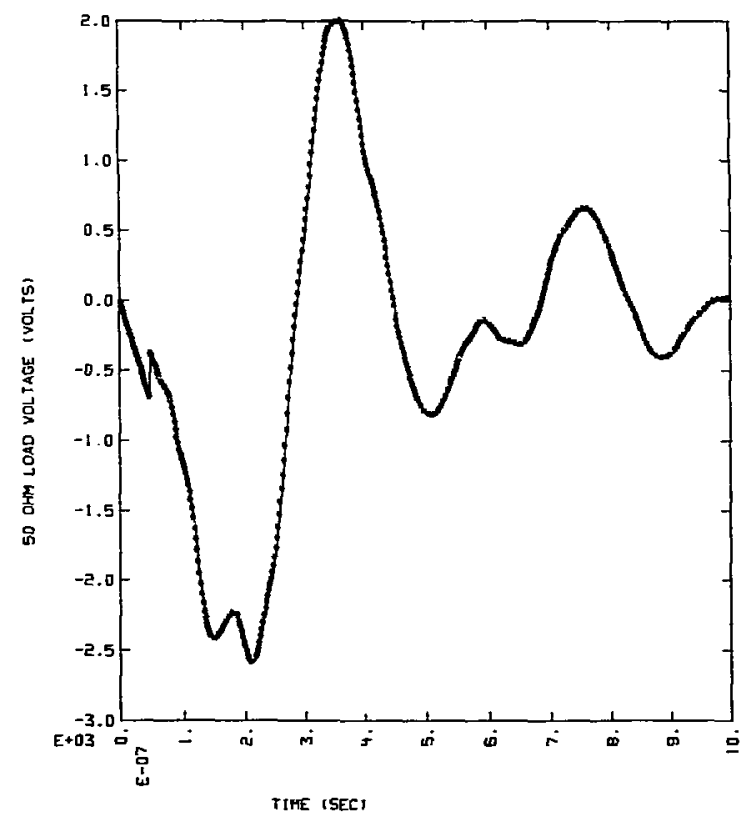

FIG. 6.1lb. Predicted full-scale transient response - AN/SRC-23 flat top (starboard). 


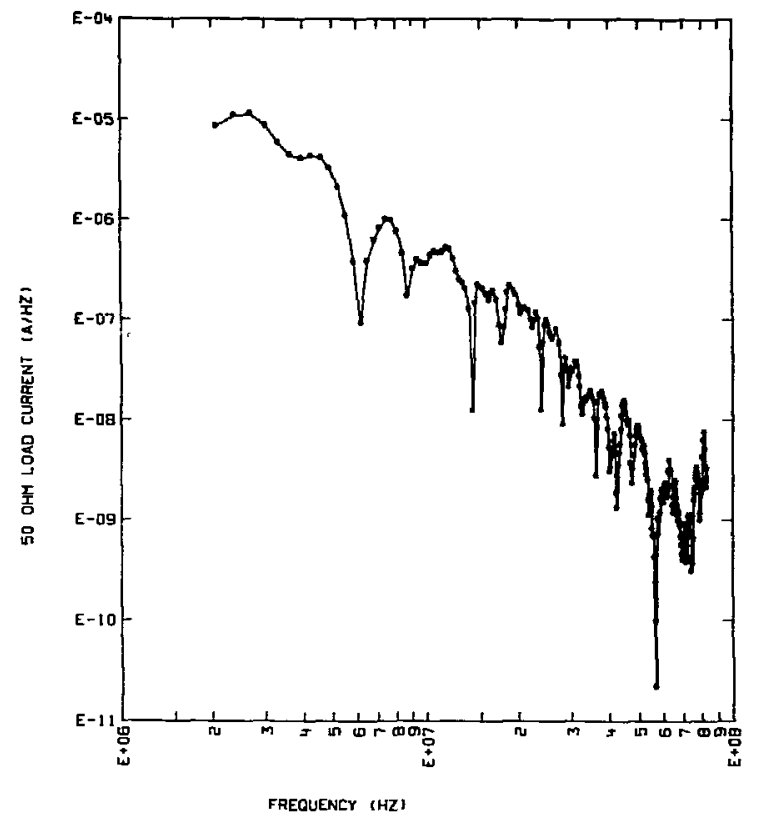

FIG. 6.1lc. Predicted full-scale frequency respnnse spectrum - AN/SRC-23 flat top (starboard).

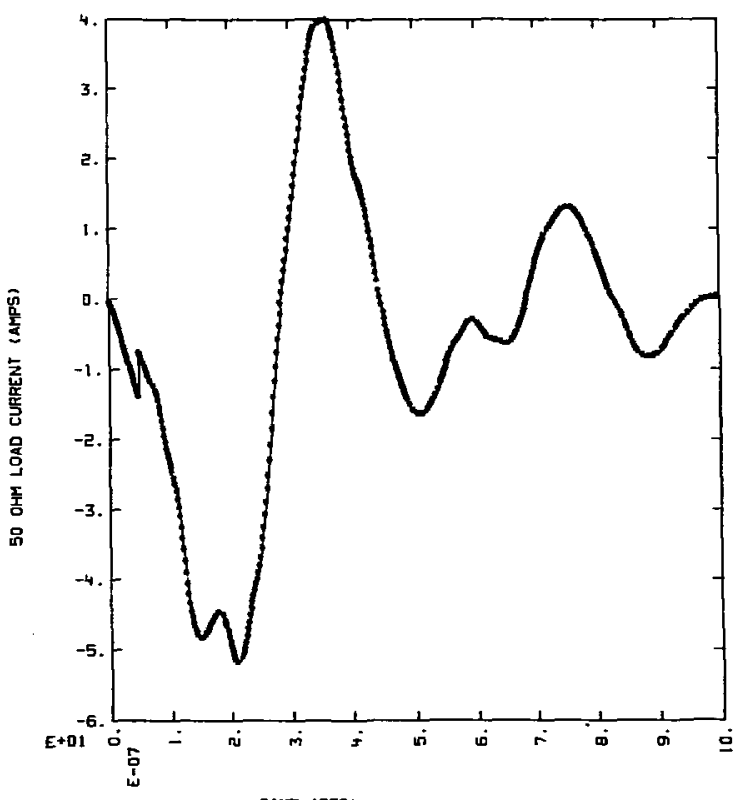

TIHE (5EC)

FIG. 6.11d. Predicted full-scale transient response - AN/SRC-23 flat top (starboard). 


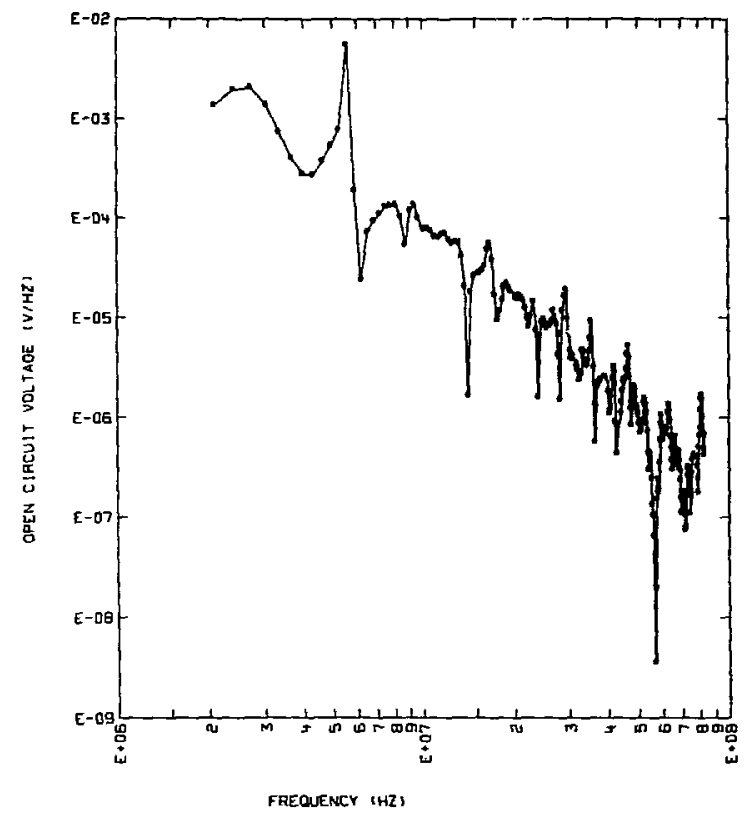

FIG. 6.1le. Predicted full-scale frequency response spectrum - AN/SRC-23 flat top (starboard).

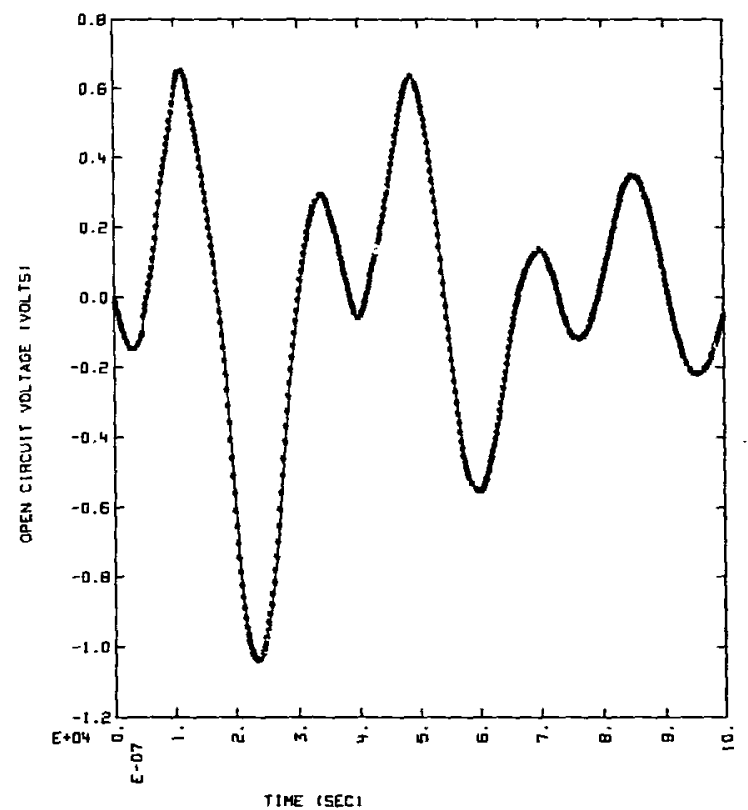

FIG. 6.11f. Predicted full-scale transient response - AN/SRC-23 flat too (starboard). 


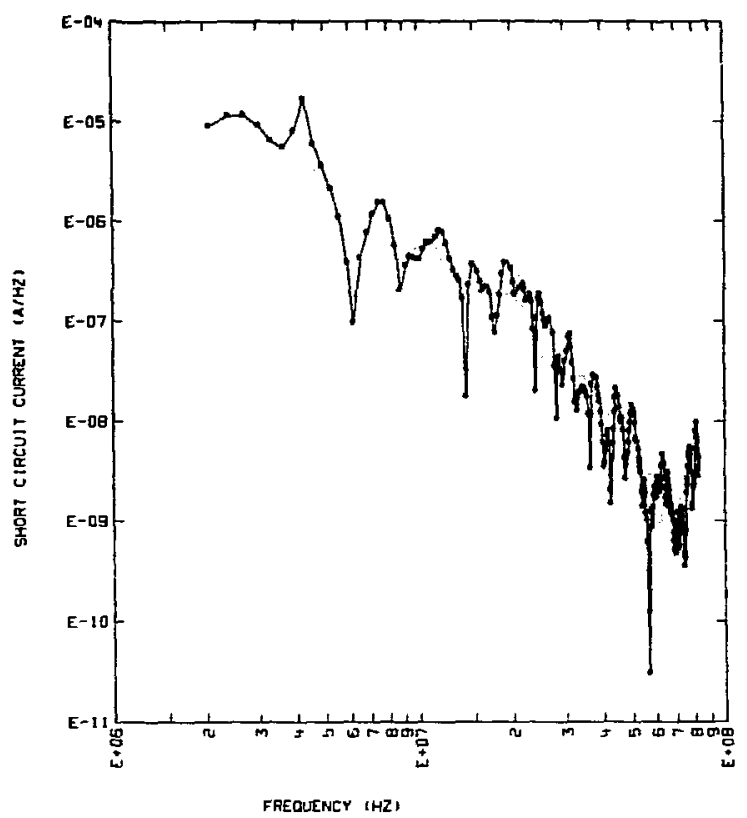

FIG. 6.1lg. Predicted full-scale frequency response spectrum - AN/SRC-23 flat top (starboard).

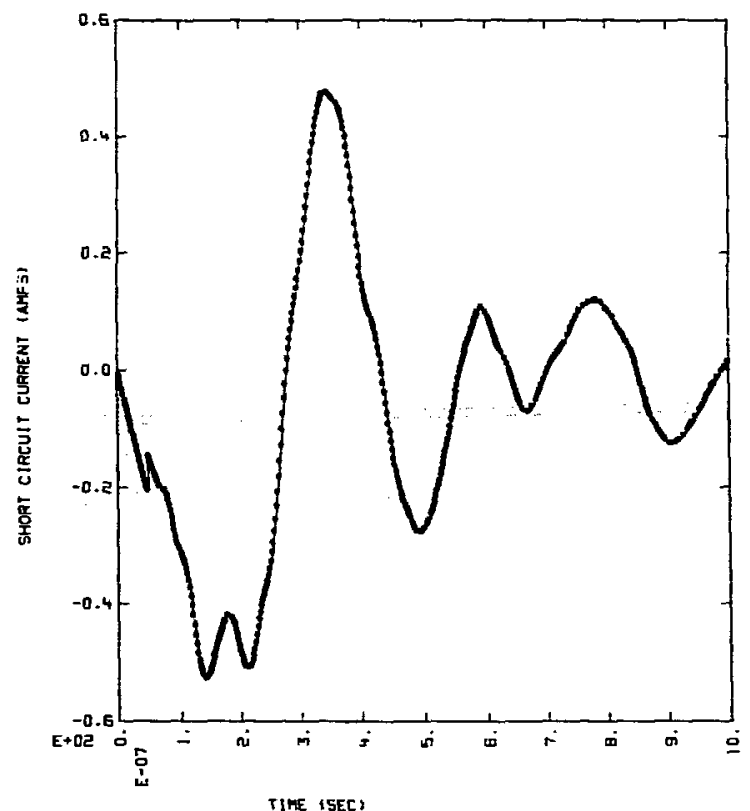

FIG. 6.11h. Predicted full-scale transient response - AN/SRC-23 flat top (starboard). 


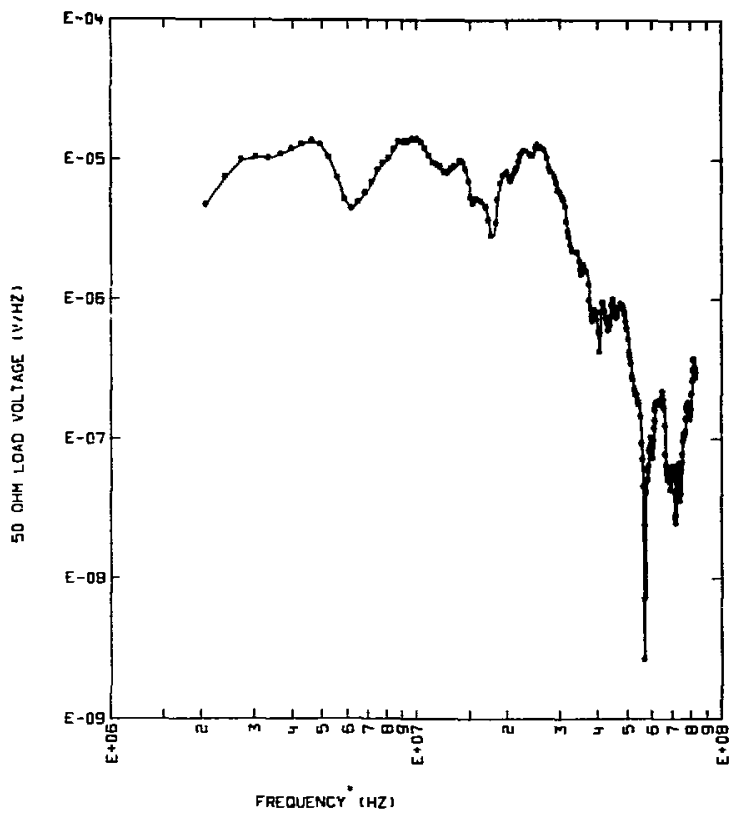

FIG. 6.1.2a. Predicted full-scale frequency response spectrum - AN/SRN-12 Omega.

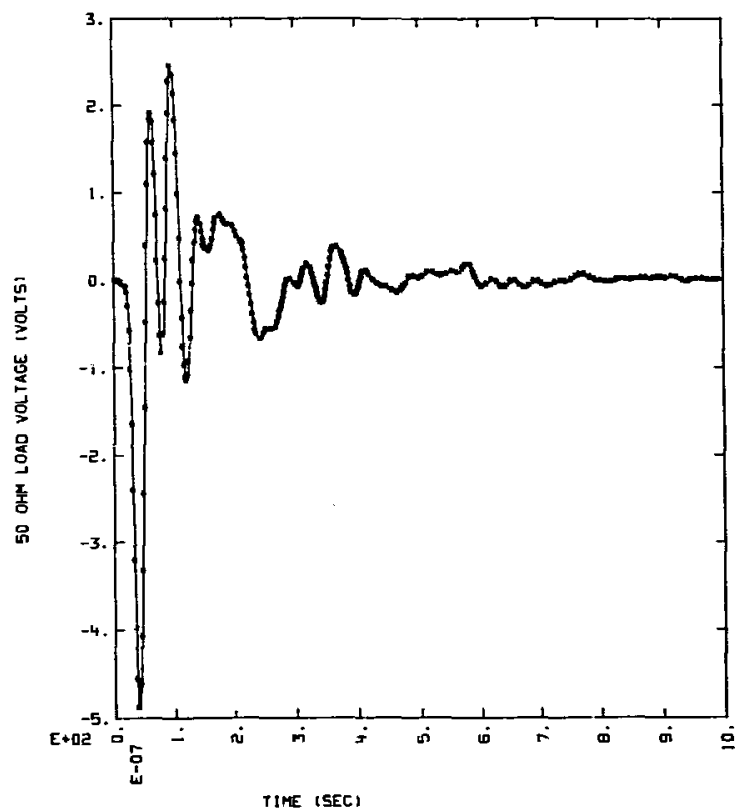

FIG. 6.12b. Predicted ful1-scale transient response - AN/SRN-12 Omega. 


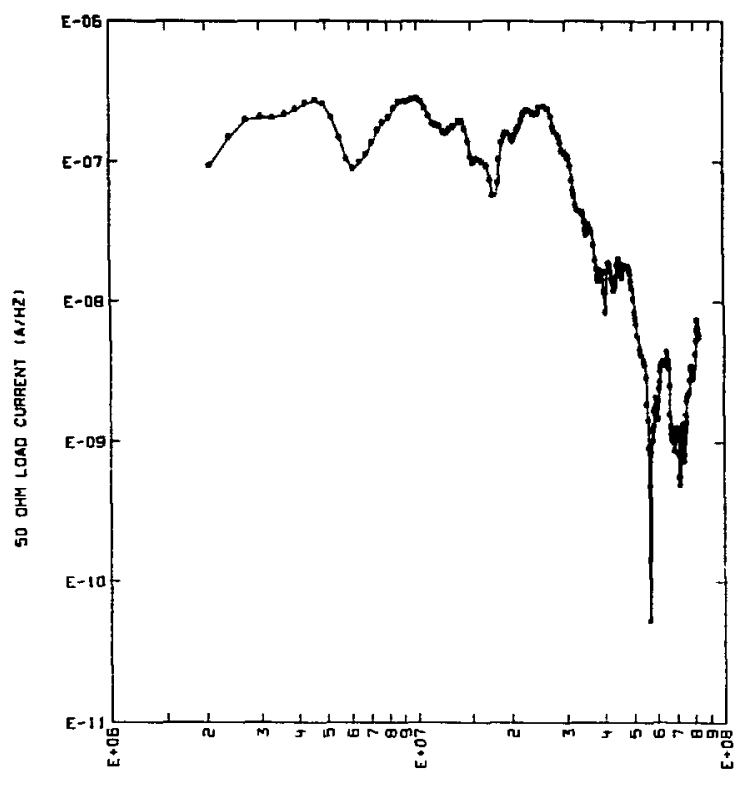

FAEOUENCY $1 \mathrm{HZ}$ :

FIG. 6.12c. Predicted full-scale frequency response spectrum - AN/SRN-12 Omega.

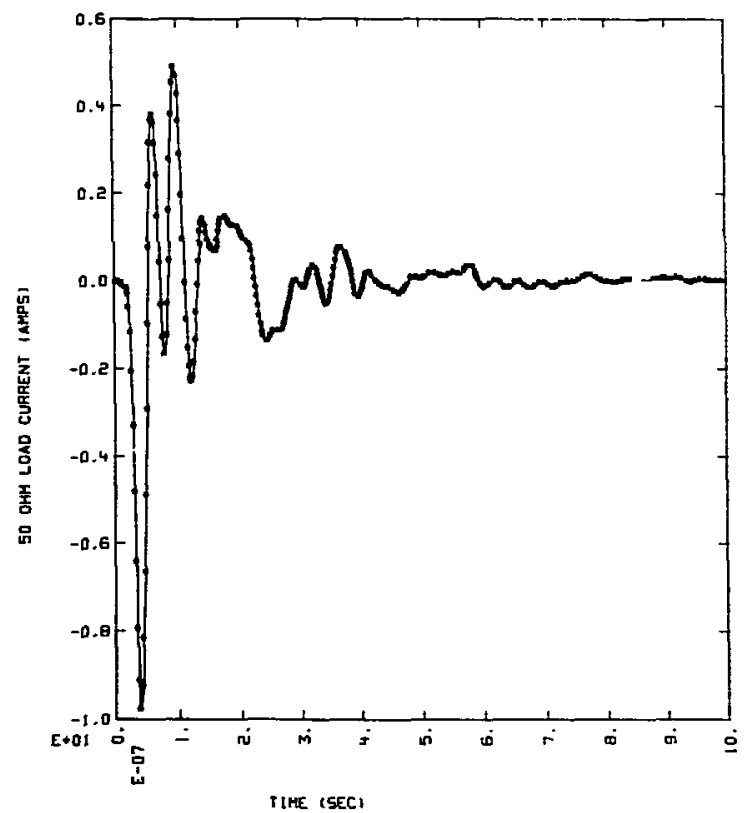

FIG. 6.12ā. Predicted full-scale transient response - AN/SRN-12 Omega.

6-35 


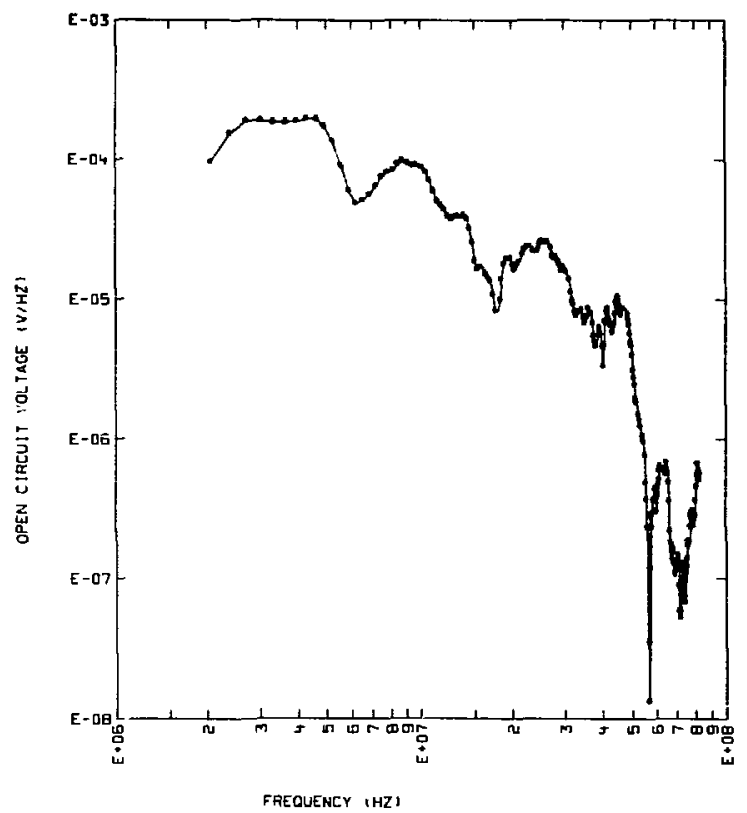

FIG. 6.12e. Predicted full-scale frequency response spectrum - AN/SRN-12 Omega.

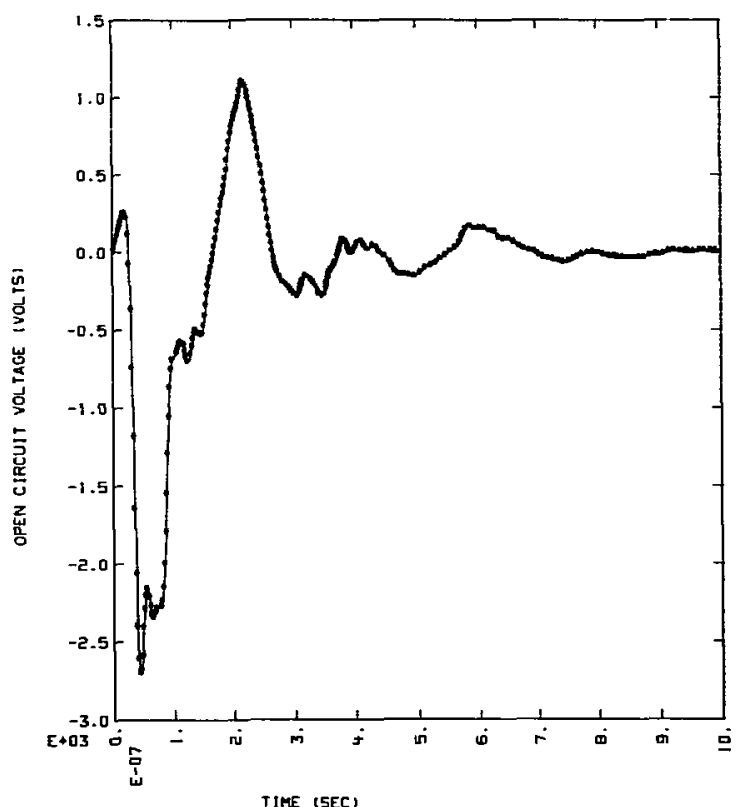

FIG. 6.12f. Predicted full-scale transient response - AN/SRN-12 Omega. 6-36 


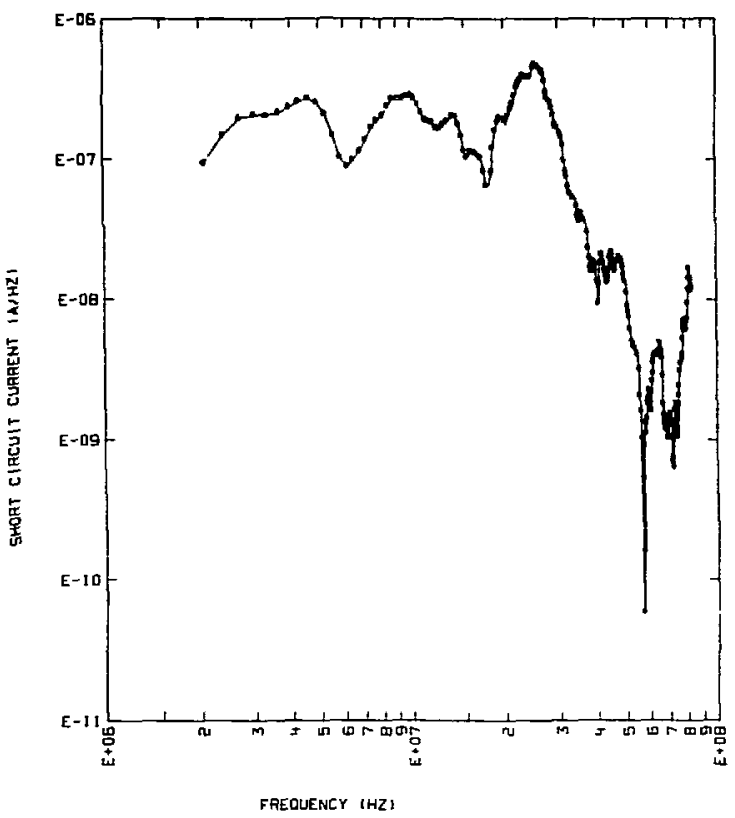

FIG. 6.12g. Predicted ful1-scale frequency response spectrum - AN/SRN-12 Omega.

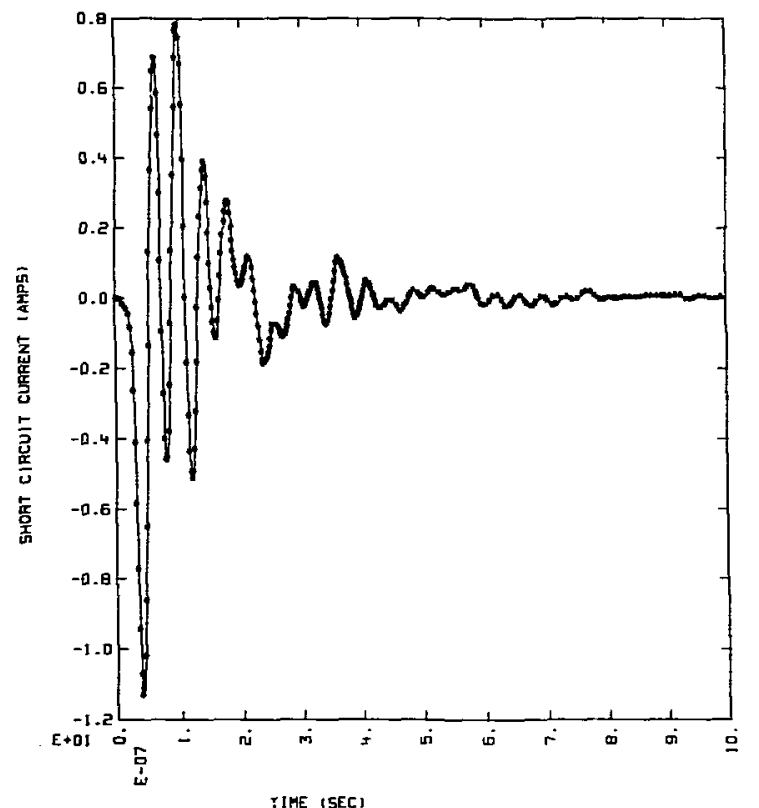

FIG. 6.12h. Predicted full-scale transient response - AN/SRN-12 Omega. 


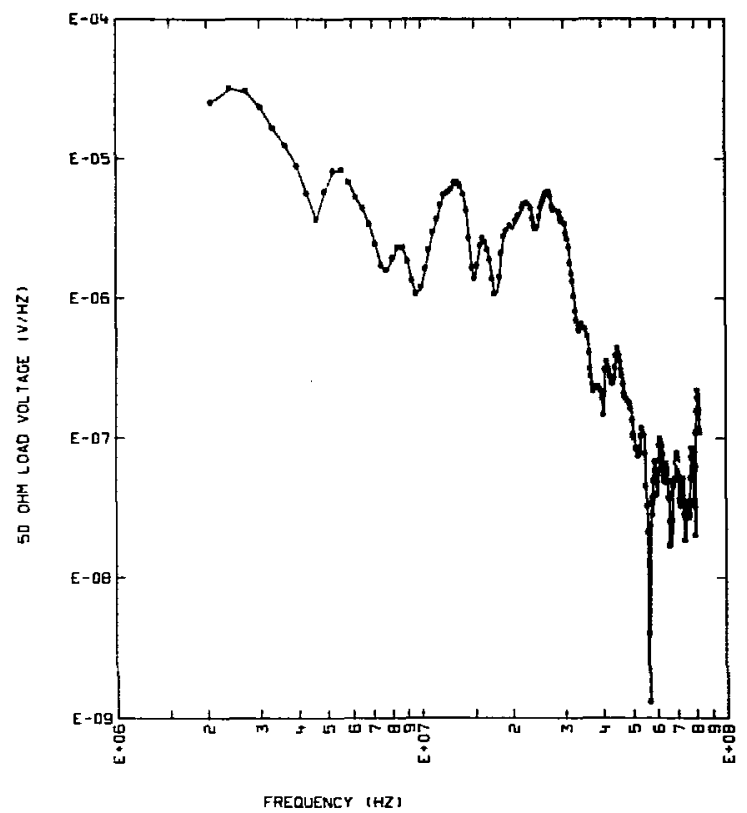

EIG. 6.13a. Predicted full-scale frequency response spectrum - AT-924B/AN/ SRA-17c (port).

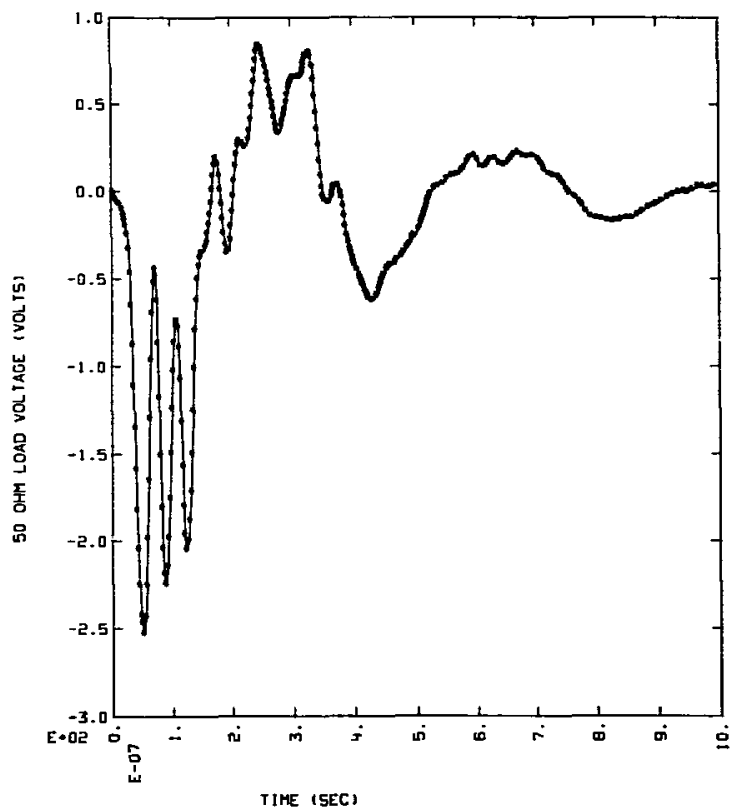

FIG. 6.13b. Predicted Eull-scale transient response - AT-924B/AN/SRA-17C (port). 


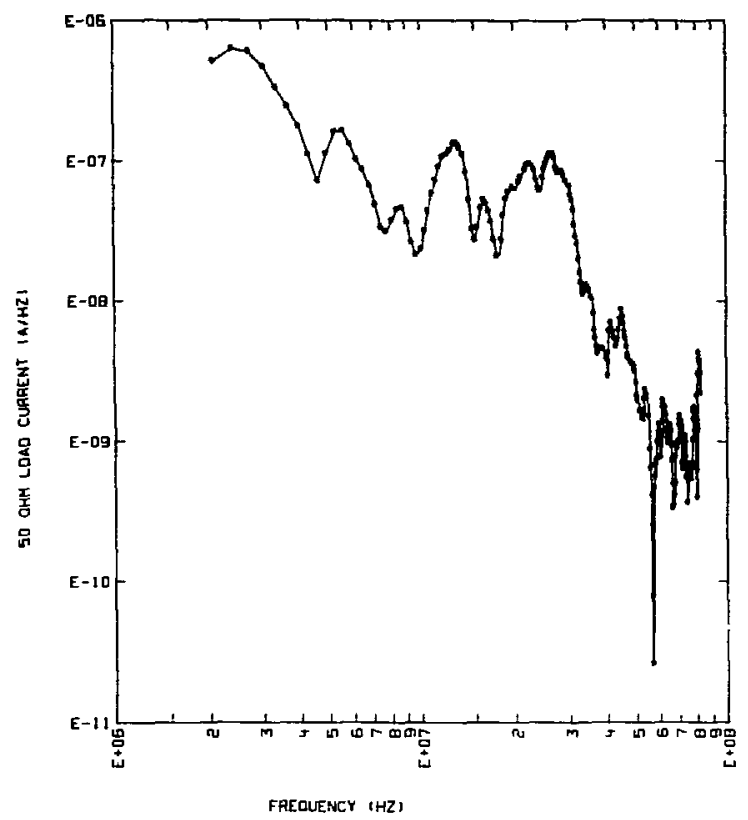

FIG. 6.13c. Predicted full-scale frequency response spectrum - AT-924B/AN/ SRA-17c (port).

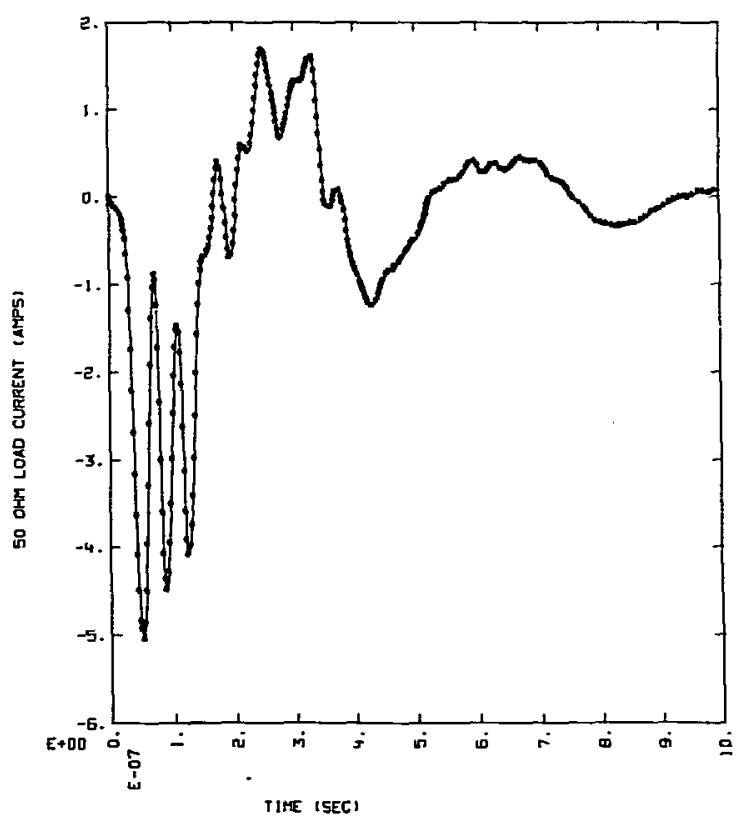

FIG. 6.13d. Preaicted full-scale transient response - AT-924B/AN/SRA-17C (port). 


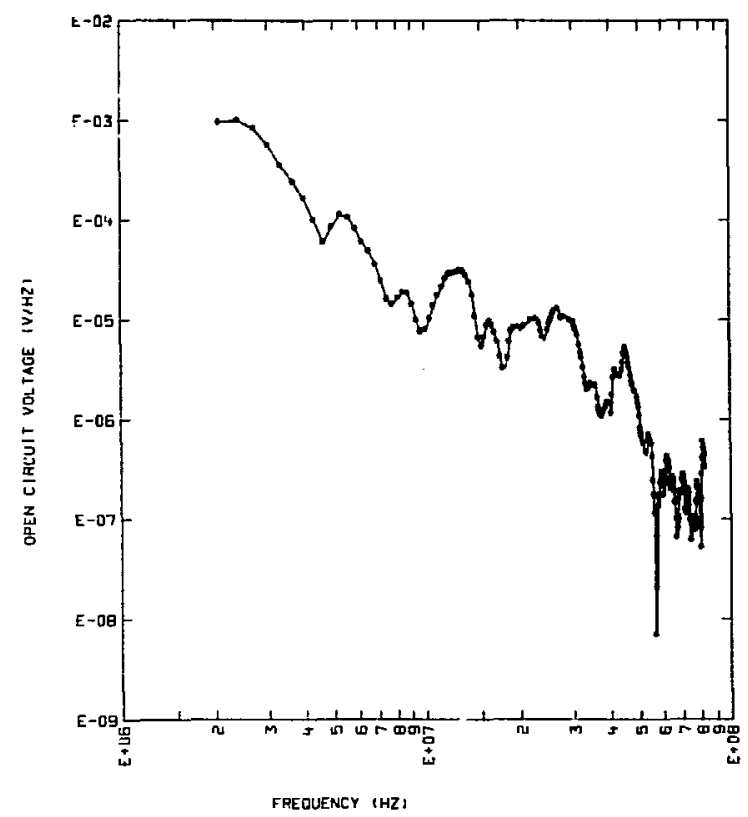

FIG. 6.13e. Predicted ful1-scale frequency response spectrum - AT-924B/AN/ SRA-17c (port).

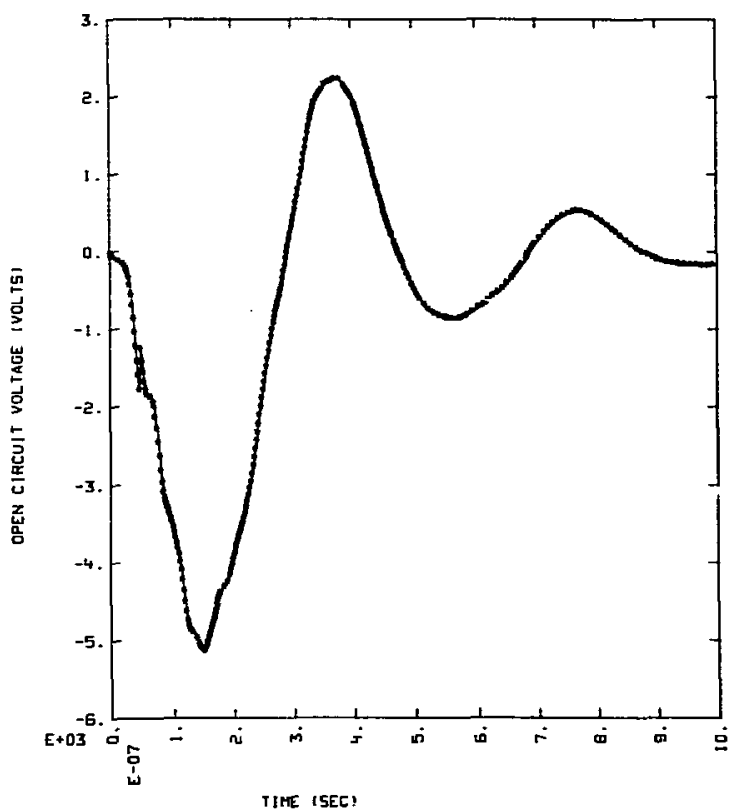

FIG. 6.13E. Predicted full-scale transient response - AT-924B/AN/SRA-17C (port). 


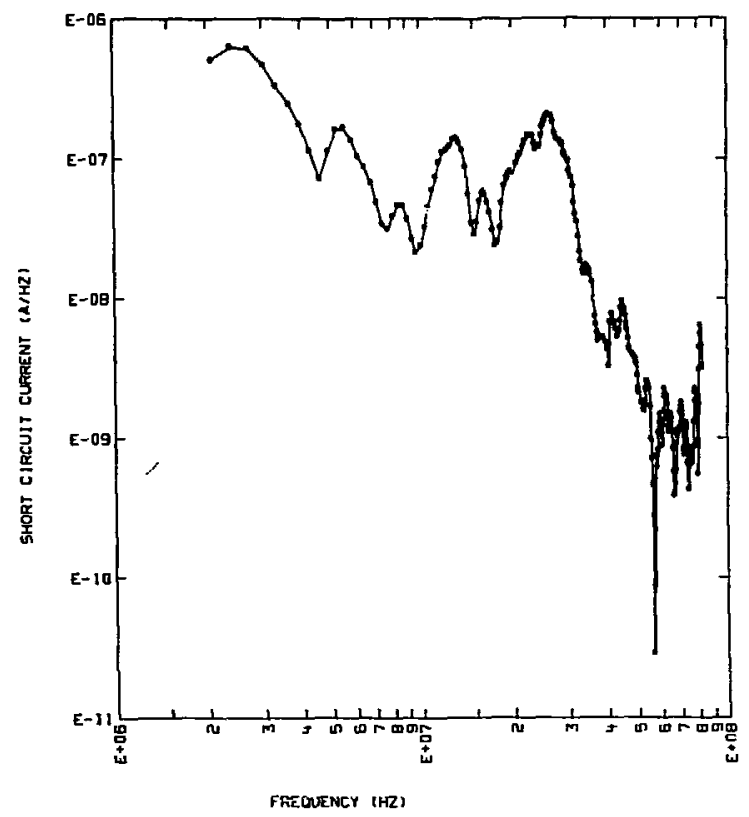

FIG. 6.13g. Predicted full-scale frequency response spectrum - AT-924B/AN/ SRA-17c (porí).

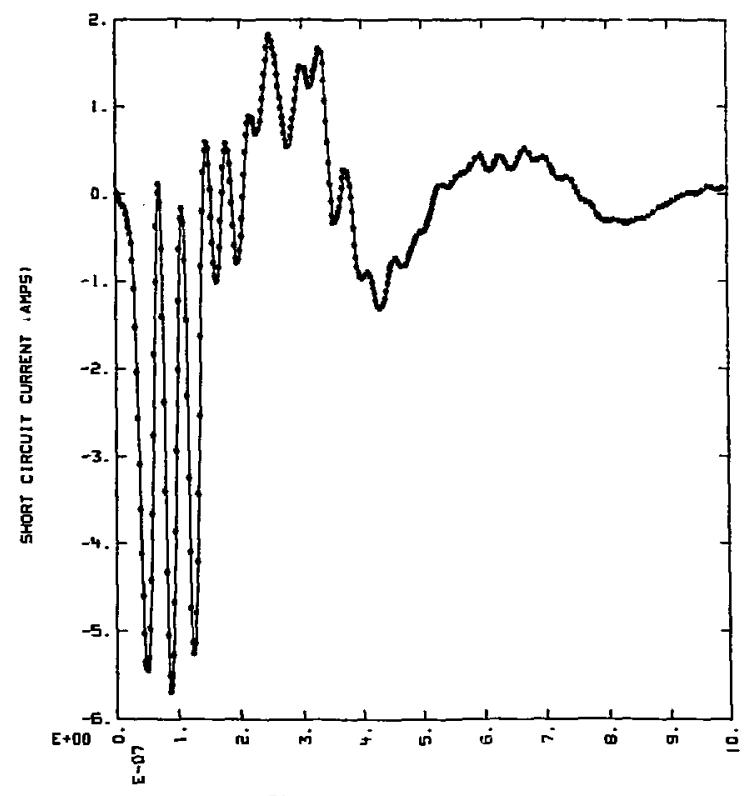

TIHE ISEC)

FIG. 6.13h. Predicted full-scale transient response - AT-924B/AN/SRA-17C (port). 


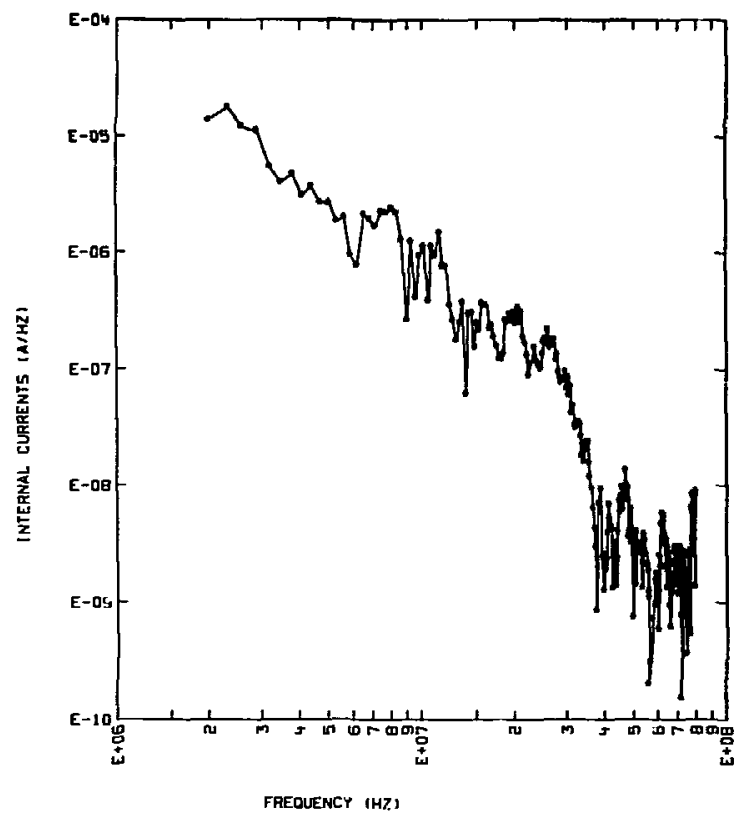

FIG. 6.14a. Predicted full-scale mast current spectrum between levels $A$ and $B$ (forward port).

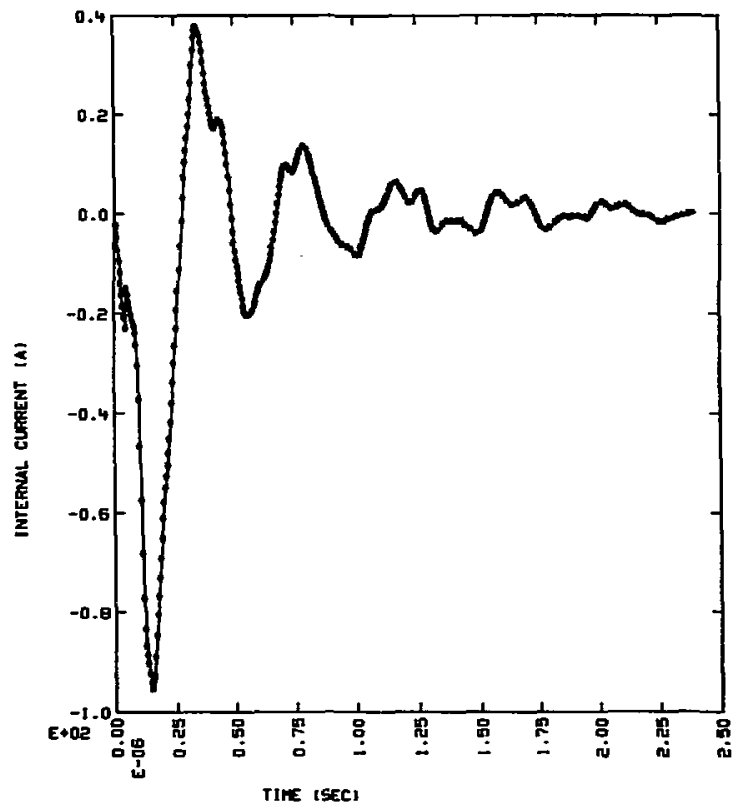

FIG. 6.14b. Predicted full-scale transient mast current between levels A and B (forward port). 


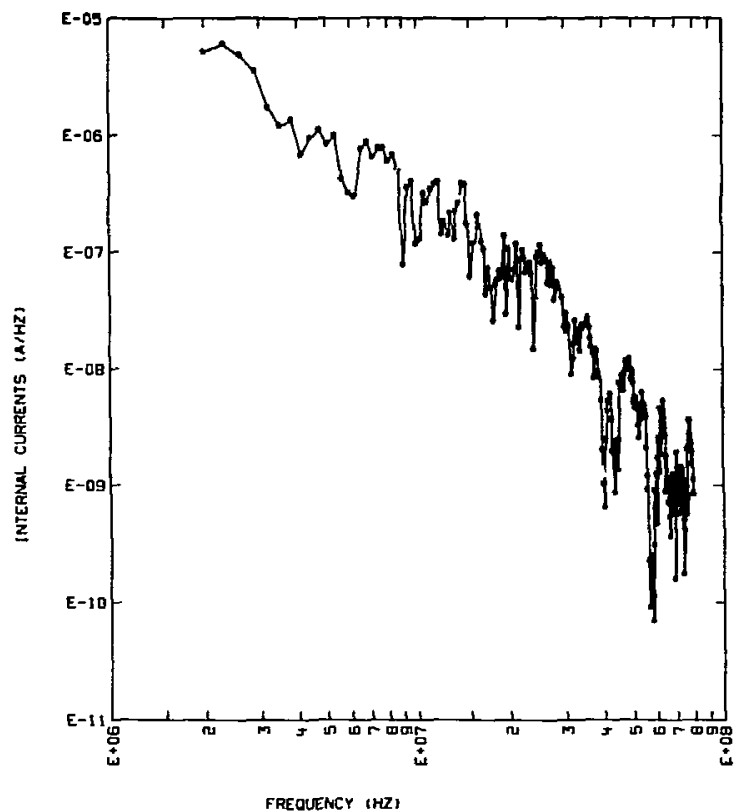

FIG. 6.15a. Predicted full-scale mast current spectrum between Ievels $C$ and $D$ (Forward port).

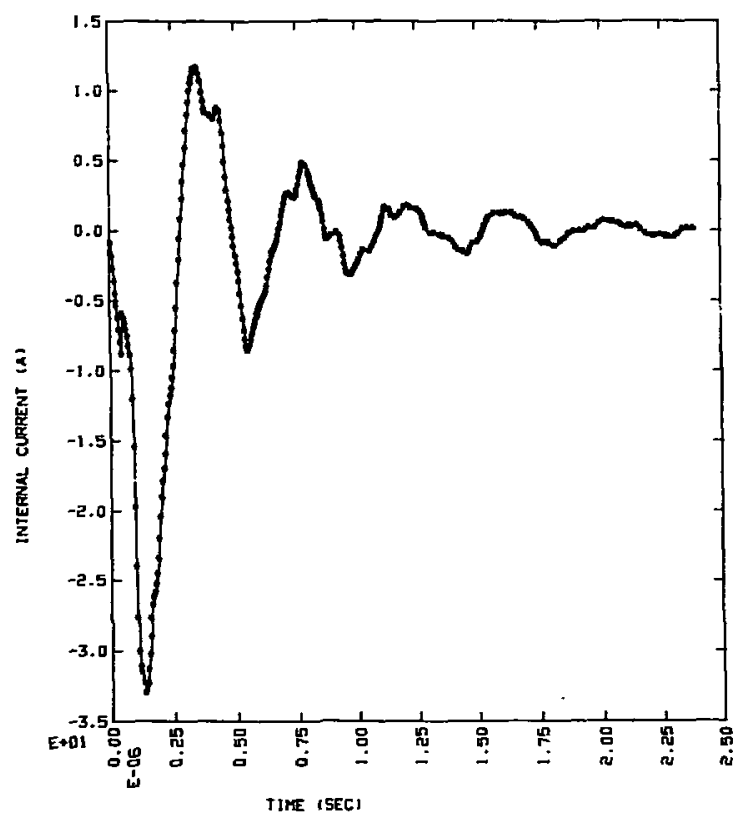

FIG. 6.15b. Predicted full-scale mast current between levels $C$ and D (forward port). 


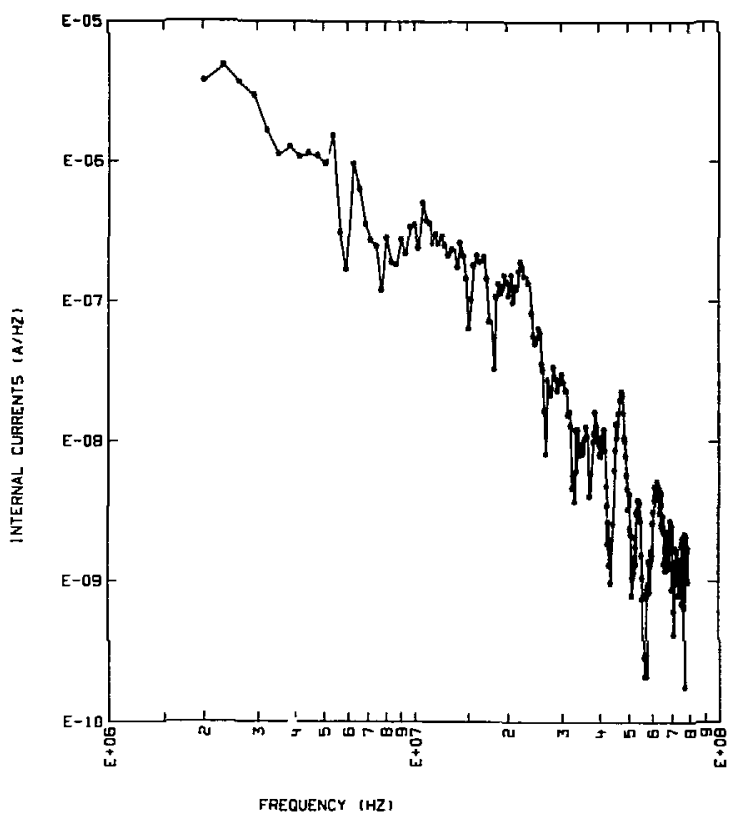

FIG. 6.16a. Predicted full-scale mast current spectrum between levels G and H.

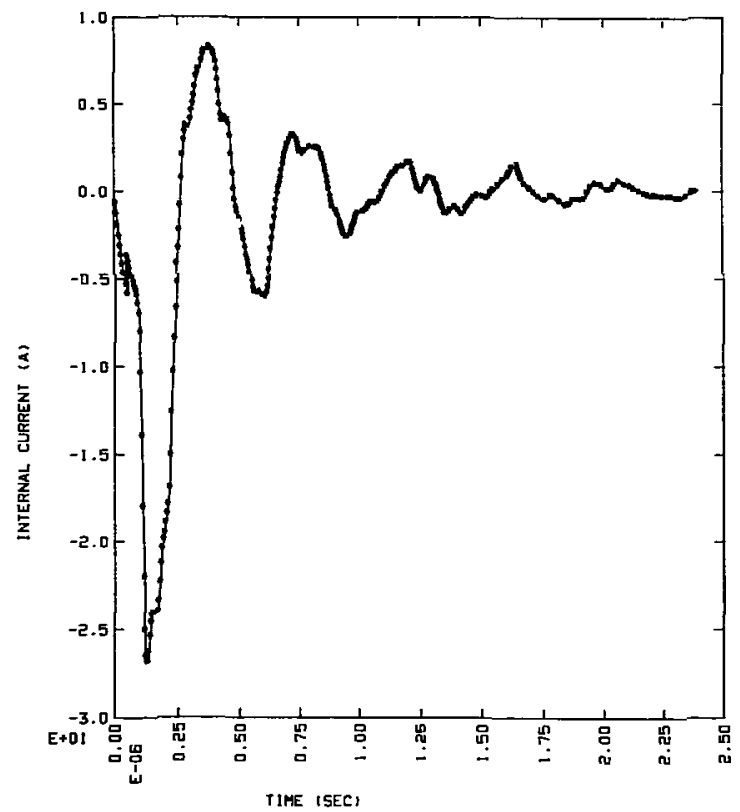

FiG. 6.16b. Predicted full-scale mast current between levels $\mathrm{G}$ and $\mathrm{H}$. 


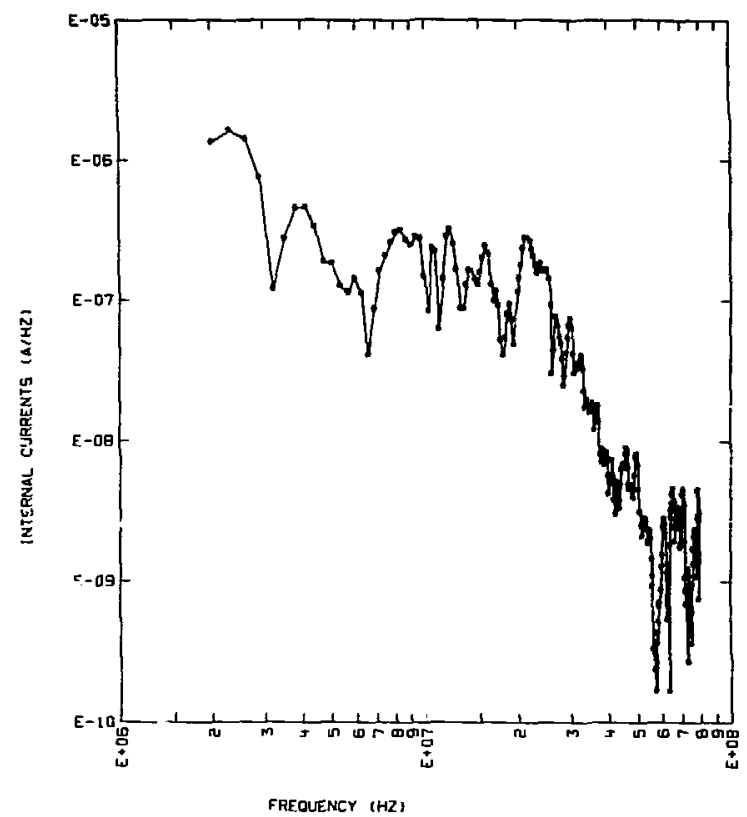

FIG. 6.17a. Predicted full-scale mast current spectrum on horizontal strut at level $G$.

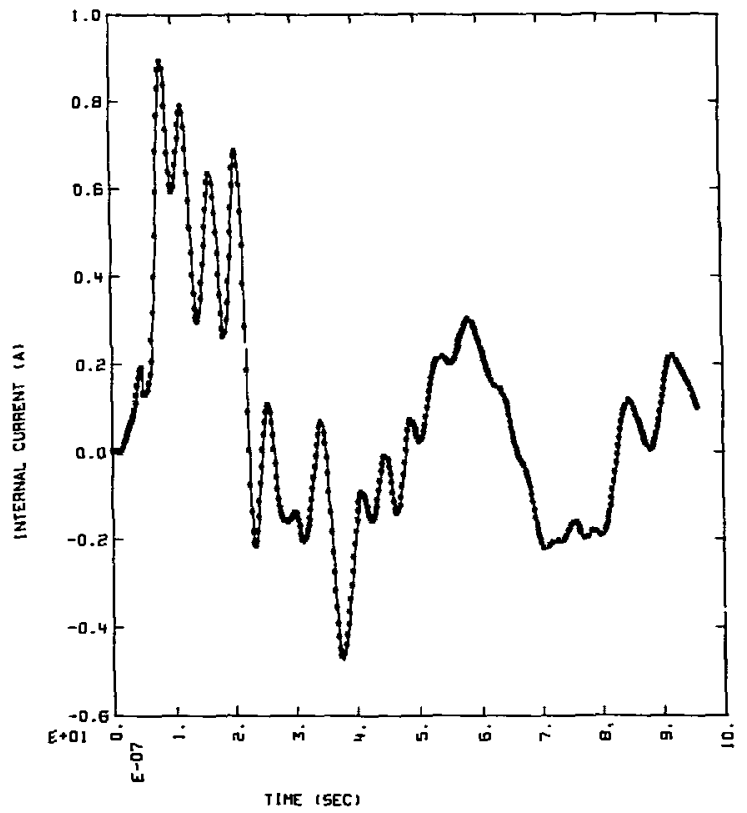

FIG. 6.17b. Predicted full-scale mast current on horizontal strut at level G. 


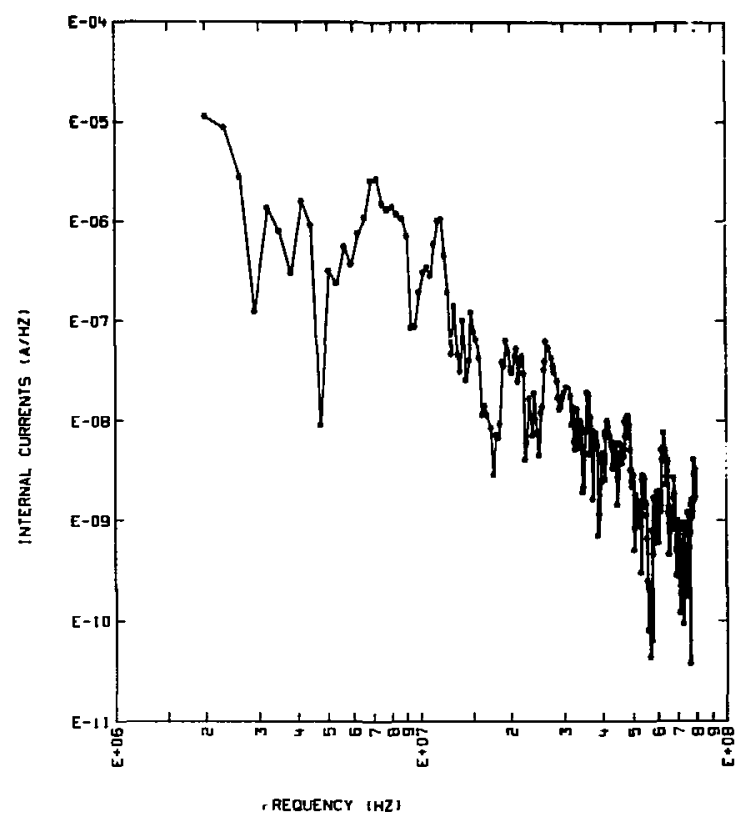

FIG. 6.18a. Predicted full-scale current spectrum on EWER cable.

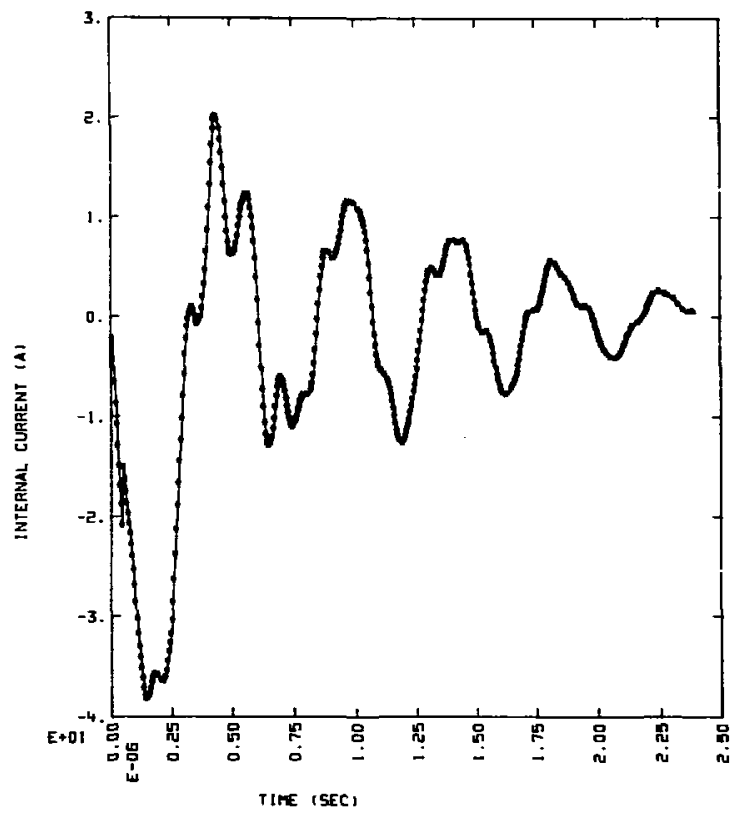

FIG. 6.18b. Predicted full-scale transient current on ENER cable. 


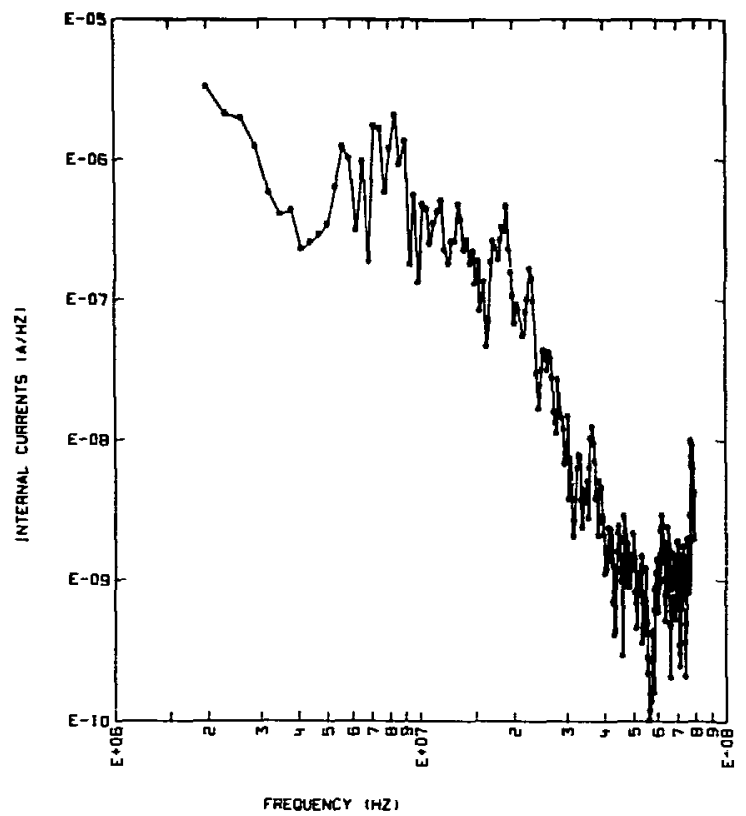

FIG. 6.19a. Predicted full-scale current spectrum on EWCR cable.

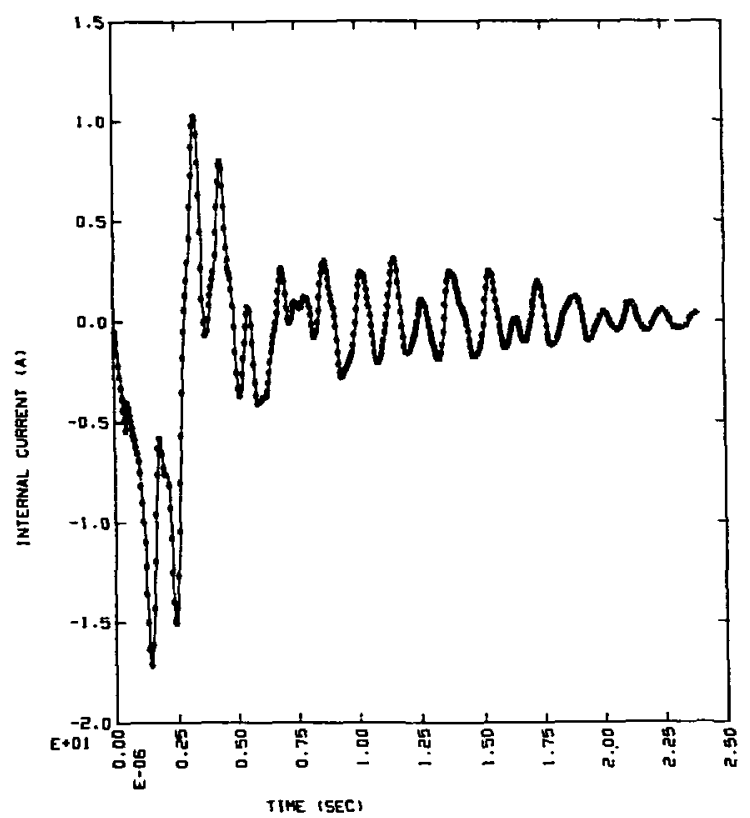

FIG. 6.19b. Predicted full-scale transient current on ExCR cable. 


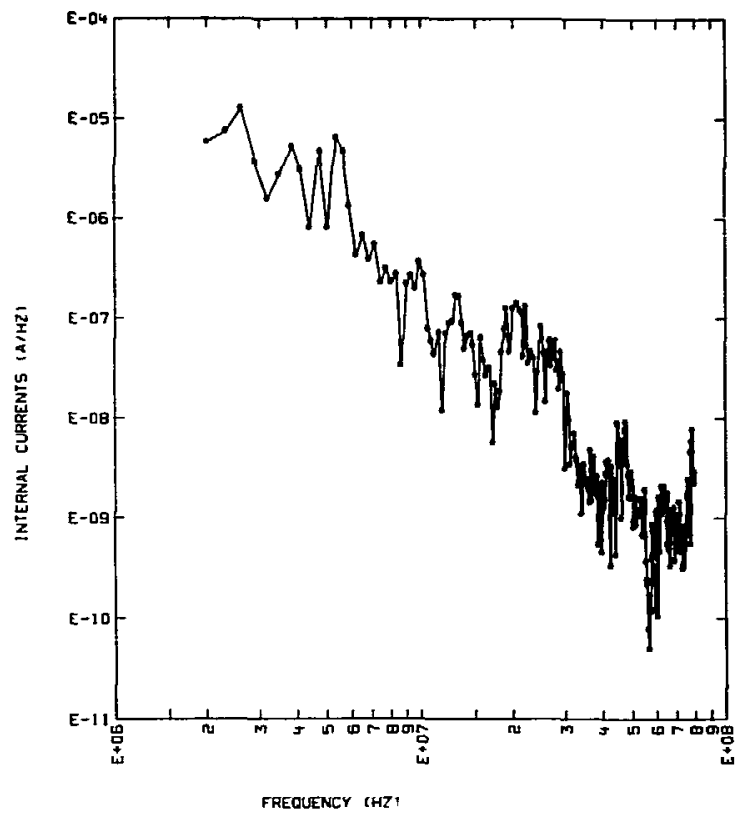

FIG. 6.20a. Predicted full-scale current spectrum on TACAN room cable.

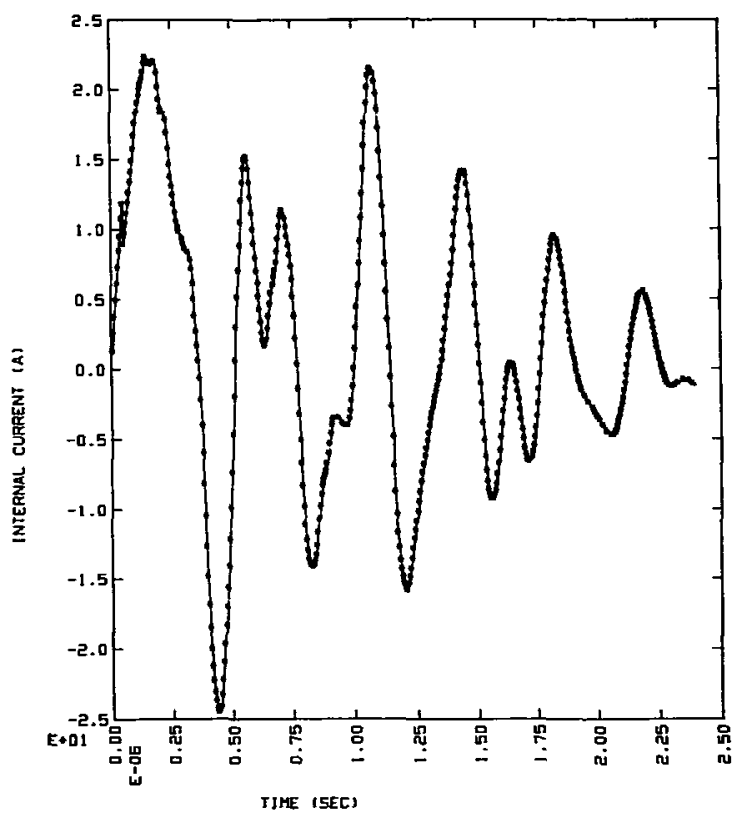

FIG. 6.20b. Predicted full-scale transient current on TACAN roam cable. 


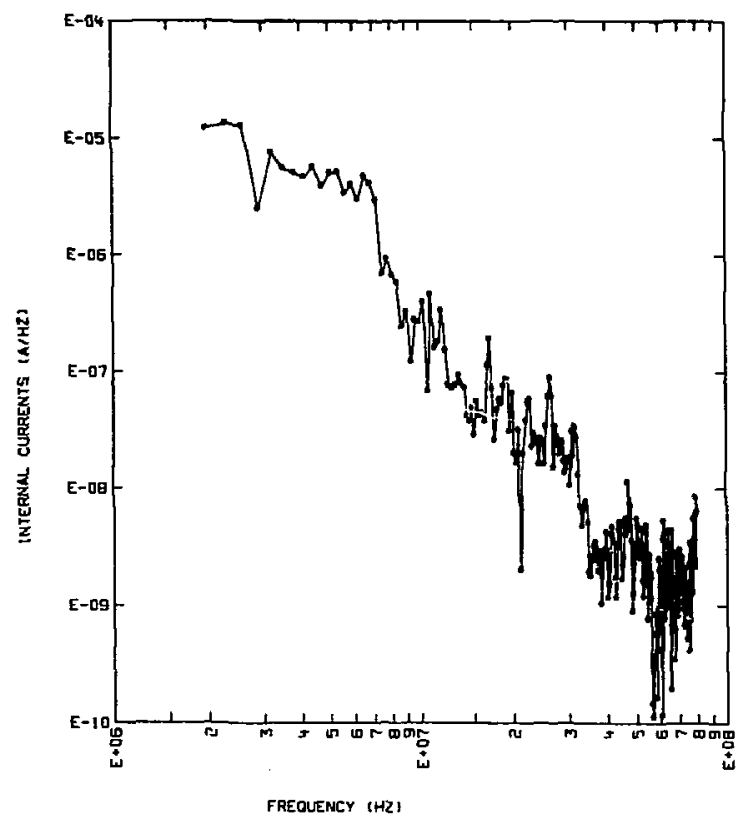

FIG. 6.2la. Predicted full-scale curcent spectrum on TX-1 room cable.

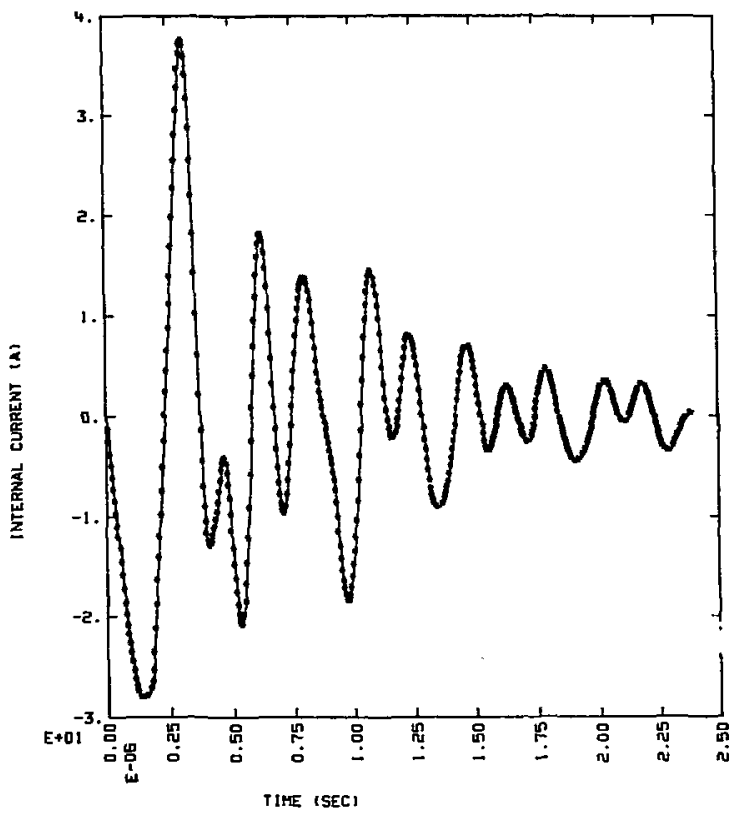

FIG. 6.2lb. Predicted full-scale transient current on TX-1 room cable. 


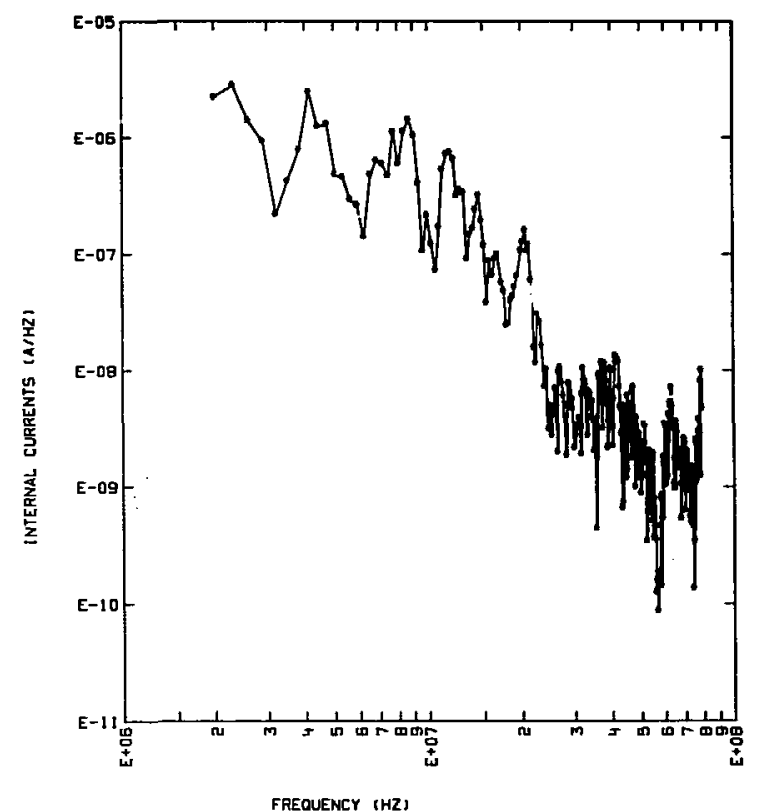

FIG. 6.22a. Predicted full-scale current spectrum on TX-2 room cable.

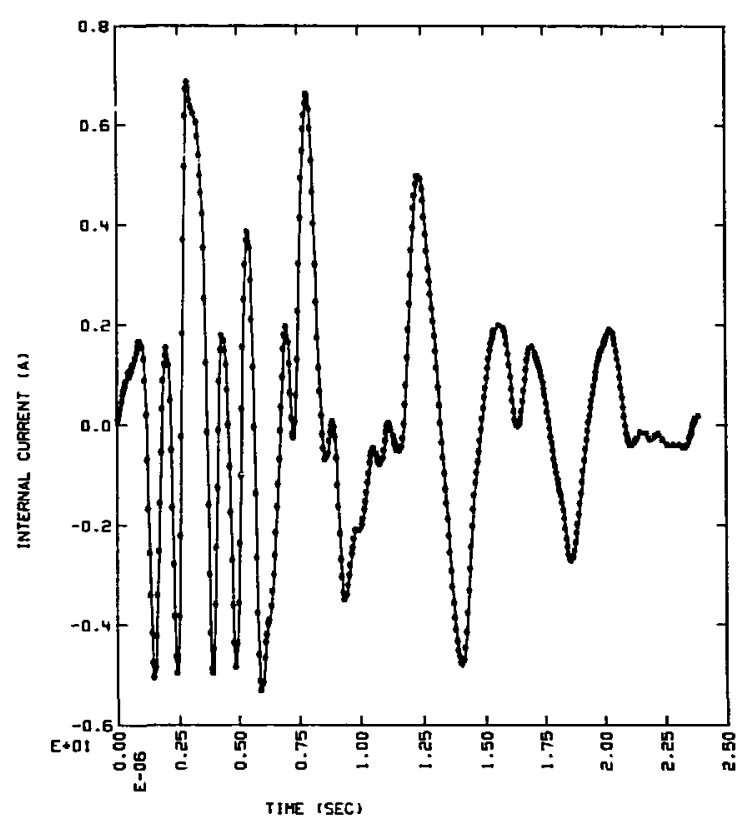

FIG. 6.22b. Predicted full-scale transient current on TX-2 room cable. 


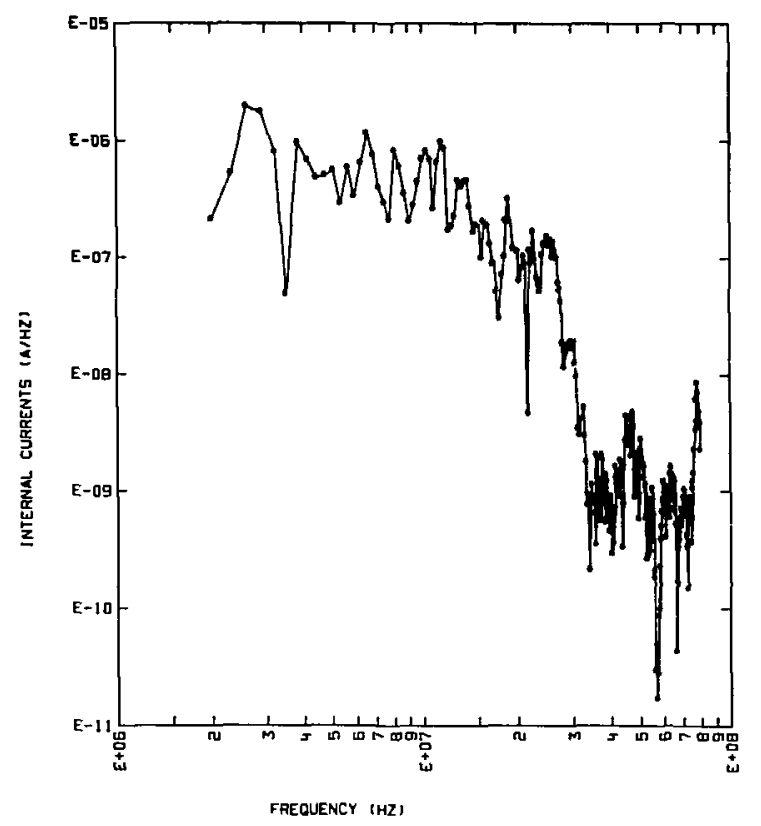

FIG. 6.23a. Predicted full-scale current spectrum on CCR cable.

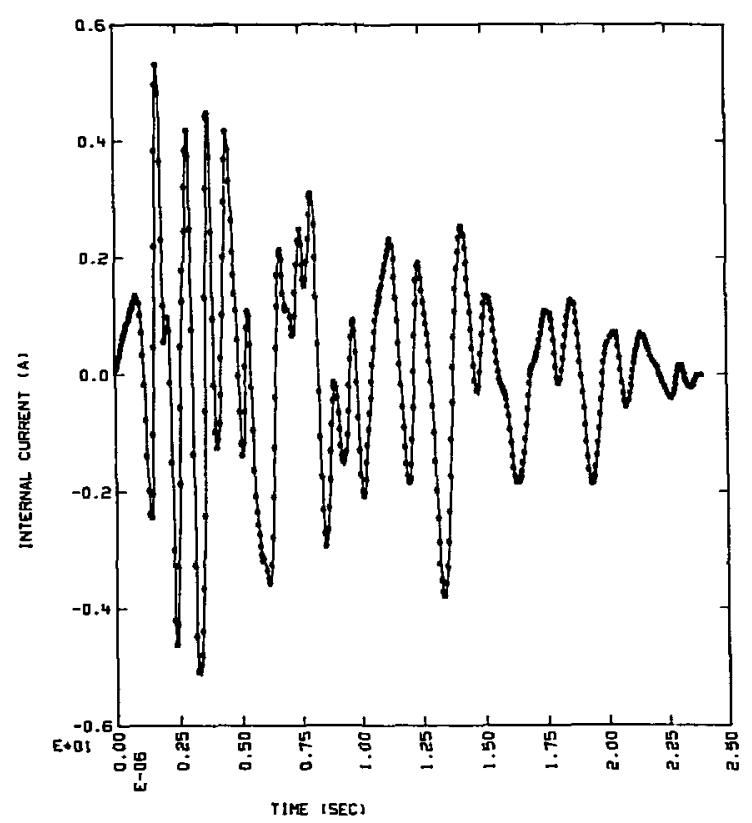

FIG. 6.23b. Predicted full-scale transient current on CCR cable. 


\subsection{CONCLUSIONS AND RECOMMENDATIONS}

Conclusions regarding the extensive use of the results would be premature since we have not had an opportunity to make comparisons with the measurements made by NSWC on the full-scale ship. We do believe, however, that the complete set of predictions for values of an EMP induced environment on elements external to the ship (antenna elements, mast, cables, etc.) will be sufficient For developing the equivalent sources for hardening purposes. That is, rather than full-scale test a ship merely to determine the coupling to external elements, it would be more efficient to make predictions of these values from extensive tests on scale models and then go to the ship with appropriate signal injection gear to check out the specific penetrations to interior circuits.

The scale-model tests in conjunction with the data base from the EMP coupling data modules developed by LLL for DNA should provide the necessary information for source model development and, at the same time, permit the study of particular configurations with relative ease and at minimum cost. The exact limits of scale modeling have not yet been explored, although the practical limits are very nearly those which we have encountered in the present prediction task.

Clearly, many of the uncertainties we had regarding the construction of the model would have been answered by an examination of the actual ship. Although we obtained reasonably complete data from NSWC on the external configuration, the interior configuration was largely a guess. Further comments will have to await a comparison with the full-scale tests. (See Appendix A.) 


\section{APPENDIX A: \\ A COMPARISON OF NSWC MEASUREMENTS AND LLL PREDICTIONS \\ OF THE EMP RESPONSE OF THE HMCS HURON}

\section{A. 1.0 INTRODUCTION}

When performing an experimental assessment of a system's response to EMP excitation--be the system a ship, aircraft, or missile--it is always advantageous to have good quality pretest predictions or estimates of the responses that will be experimentally induced. The advantages of good quality pretest predictions are related to the fact that the responses are in some sense already known before the experiment commences. Thus the experimental program's recording requirements--such as types of probes, sweep speeds, record length, etc.--needed to resolve important features of the data can be addressed. Also the pretest predictions may reveal unanticipated responses that may then require the experimental program to be modified so that these responses, if they occur as expected during the experiment, can be examined in more detail.

Errors in a measurement can be detected when large discrepancies exist between a prediction and a measurement. Of course in some instances the error will be found in the prediction, but, when gooa predictions are available, it is the measurement that often is at fault.

There are many sources of pretest predictions in the form of various numerical codes. A representative example of a numerical code pretest predictor is given by Ref. 1. In this example and with many other codes, fair to very good agreement between measurement and prediction is obtained over a reasonably broad frequency range for systems as complex as an aircraft. However, such codes have not in general been applied to such large objects as ships because the detail that must by modeled when interior responses are required is too fine to be accomodated from these codes.

Scale modeling is the other major avenue for obtaining pretest response predictions. It has been employed successfully for aircraft systems ${ }^{2}$ and should be capable of similar success when applied to ships.

The LIL 1/48-scale model of the HMCs Huron tested at LLL's vertical EM simulator test range provided an opportunity to compare model results with actual ship responses as obtained by Maval Surface Weapons Center (NSWC) on the Hucs Huron at the EMPRESS facility. 
The coupling predictions were grouped by WSWC into three categories: (1) mast structural elements, (2) interior cables, and (3) antenna center conductors. The same categories are used here.

Meaningfui comparisons are difficult for these three categories in that differences between predicted and measured data may often be the result of differences in location and not the result of model shortcomings; therefore, only the cases where no location discrepancies or ambiguities exist are examined.

A brief assessment of the model predictions follows the LLL/NSWC comparisons.

\section{A. 2.0 NSWC AND LLL TIME-DOMAIN RESPONSE COMPARISONS}

\section{A. 2.1 MAST STRUCTURAL ELEMENTS RESPONSES}

Table A.1 gives the mast data measurement locations. Figures A.1, A.3, A. 5, and A. 7 compare the time-domain responses of the measured and predicted data at each location followed by Figs. A. 2, A.4, A.6, and A.8, the Fourier transforms of the LLL data. No frequency domain data are available for the NSWC measurements; however, the transforms of the ILL predictions are helpful in developing insight into the corresponding time-domain data.

Table A.2 summarizes the mast structural element response comparisons. The NSWC data were adjusted for a GENISCO 8-in. current probe with sensitivity of $1 \mathrm{~mA}: 2.8 \mathrm{mV}$. The LLL predictions were already corrected for the scale and difference between the scaled LLL waveform and the NSwC waveform (the transfer function between the electric fields of the two waveforms was applied to the model measurements).

The frequency range estimates for the NSWC and LLL data are based on some very heuristic yet practical principles. Table A. 2 shows that only the relatively low-lying frequency components can be resolved and compared. At the high end, the limiting factor is the experimental data. (Faster sweep 
TABLE A.1. Mast structural element data measurement locations.

\begin{tabular}{lcc}
\hline & \multicolumn{2}{c}{ NSWC measurement } \\
\cline { 2 - 3 } Measurement location & Test point & Shot no. \\
\hline Horiz. cross member at G & $M-17$ & 1070 \\
Forward port leg between G and H & $M-13$ & 1002 \\
Forward port leg between C and D & $M-49$ & 966 \\
Forward port between A and B & $M-55$ & 116 \\
\hline
\end{tabular}

speeds and signal conditioning are needed if responses up to $50 \mathrm{MHz}$ are to be resolved.) At the low end, the limiting factor is the record length of the predictions. (Record length is limited by the clear time of the LLL test facility and can not be readily extended. This prevents very low-frequency components fron being picked up in the predictions.) However, according to Dr. Libelo of NSWC, the only significant verv low-frequency signals seen in the measurements, and not predicted, were the result of a signal internally generated by the ship.

\section{A.2.2 INTERIOR CABLE RESPONSES}

According to NSWC several cables from the compartments to the various antennas on the mast were not accurately modeled. Table A.3 gives NSWC's assessment of these discrepancies. Based on this assessment the electronic warfare control room (EKCR) and the radar and TACAN room (RTR) comparisons were considered fair.

For the electronic warefare equipment room (EWER), transmitter room no. 2 (TX-2), and communications control room (CCR) comparisons, multiple shots were obtained for the measurements and these exhibited variations by factors of two or greater making the data questionable. The transmitter room no. 1 (TR-1) comparison was considered questionable because of the way the cable was connected at the mast. 
TABLE A.2. Mast structural element data comparisons.

\begin{tabular}{|c|c|c|c|c|}
\hline \multirow[b]{2}{*}{ Location } & \multicolumn{2}{|l|}{ NSWC measurements } & \multicolumn{2}{|l|}{ LLL predictions } \\
\hline & $\begin{array}{c}\text { Frec comp, } \\
\mathrm{MHz}\end{array}$ & $\begin{array}{l}\text { ampl, } \\
\text { A }\end{array}$ & $\begin{array}{c}\text { Freq comp, } \\
\qquad \mathrm{MHz}\end{array}$ & $\begin{array}{c}\text { Pk ampl, } \\
\text { A }\end{array}$ \\
\hline $\begin{array}{l}\text { Horiz. cross } \\
\text { member at } G\end{array}$ & $\begin{array}{l}1.13^{\mathrm{a}}, 2.2 \\
(0.2 \mathrm{MHz}<\mathrm{f}<8 \mathrm{MHz})^{\mathrm{C}}\end{array}$ & 9.3 & $\begin{array}{l}2.2^{b}, 22.3 \\
(1 \mathrm{MHz}<\mathrm{f}<45 \mathrm{MHz}) \mathrm{d}\end{array}$ & 9.0 \\
\hline $\begin{array}{l}\text { Forward port leg } \\
\text { between } G \text { and } H\end{array}$ & $\begin{array}{l}1.13^{\mathrm{a}}, 2.0 \\
(0.2 \mathrm{MHz}<\mathrm{E}<5 \mathrm{MHz})\end{array}$ & 59 & $\begin{array}{l}2.4 \\
(0.4 \mathrm{MHz}<\mathrm{f}<22 \mathrm{MHz})\end{array}$ & 27 \\
\hline $\begin{array}{l}\text { Forward port leg } \\
\text { between } C \text { and } D\end{array}$ & $\begin{array}{l}1.1^{\mathrm{a}}, 2 \\
(0.2 \mathrm{MHz}<\mathrm{f}<5 \mathrm{MHz})\end{array}$ & 17 & $\begin{array}{l}2.4 \\
(0.4 \mathrm{MHz}<\mathrm{f}<25 \mathrm{MHz})\end{array}$ & 33 \\
\hline $\begin{array}{l}\text { Forward port } \\
\text { between } A \text { and } B\end{array}$ & $\begin{array}{l}\text { poss. pk. bet. } 2-2.5 \\
(1 \mathrm{MHz}<\mathrm{f}<10 \mathrm{MH} z)\end{array}$ & 41 & $\begin{array}{l}2.4,6.5-8 \mathrm{~b} \\
(0.4 \mathrm{MHz}<\mathrm{f}<21 \mathrm{MHz})\end{array}$ & 96 \\
\hline
\end{tabular}

\footnotetext{
a signal of approximately $1 \mathrm{MHz}$ is internally generated by the ship as a reference signal.

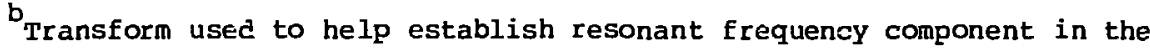
response.

Estimate of frequency range limit, $f$, is based on record length for $f$ min., where $T$ is the record length and on the oscilloscope writing rate, where 2-4 cycles/div. can be accurately written depending on amplitude, i.e., 2 cycles/ div. for a 3 div. 0-pk. extension which determines $\mathbf{f}$ max. Estimate of frequency range limit, $f$, is based on record length for $f$ min. and on a $30 \mathrm{~dB} \mathrm{~S} / \mathrm{N}$ limit in the resolvable dynamic range for $\mathrm{f}$ max., where $\mathrm{f}$ max. denotes the limit over which the prediction could be resolved experimentally using a single, unconditioned measurement limited by a $30 \mathrm{~dB}$ $S / N$ in the instrumentation. In other words, even if the prediction were completely accurate, it could only be verified up to this frequency limit with a single, unconditioned measurement.
} 
TABLE A.3. NSWC's assessment of the agreement between the model and full-scale measurements with respect to the interior cables.

Eiectronic Warfare Equipment Room (ENER) A 19-wire cable was identified and modeled by LLL as running from the AS-5058 to EWER; but, there is no such cable on the Huron. Two coaxial cables, 3R-RA9 and 3R-RAl0, connect to the As-5058 and terminate in transmitter room no. 2. These two cables are routed through EWER.

The second cable modeled was routed from level $\mathrm{J}$ on the mast down to EWER. This cable was monitored. Four Huron cables are similar to the ones modeled. These cables are connected to two AN/WIR-IC antennas at level $J$. These are cables 2R-CR-100, 2R-CR-9I, and 2R-CR-92 for the AS-899, and 2R-CR48 for the As-5050. But only two were measured during the test: ENER-01-01, shot no. 239 for the 2R-CR-100 cable and EWER-03-01, shot no. 267 for the 2R-CR-48 cabie. The waveforms were measured with a singer 51550-2 current probe with a calibration of $1 \mathrm{~mA}: 1 \mathrm{mV}$.

Transmitter Room Number Two (TX-2) There are two conflicting descriptions of the cable modeled in TX-2. One description has the cable grounded at the top platform of the mast. Another description, which is the more likely as it is mentioned twice, has the cable open ended at the top. This is due to the connection to the AS-5045 antenna which is half way up the fiberglass pole. No measurement was made on a cable connected to this antenna. Further it is not known whether the cable connected to the antenna runs into TX 2 without scrutinizing the system schematics. To provide a comparison measurement for the predicted value a cable was chosen which is similar in most respects. This cable is the 3R-RA9 or 3R-RAI0 which connect to the AS-5058 as previously mentioned. The photos of the measurements are identified as TX2-08 shot no. 1090 and TX2-07 shot no. 1109. As before, these were measured with a -2 current probe.

\section{Electronic Warfare Control Room (EWCR) There were two cables modeled} which were terminated in EWCR. The cable monitored can clearly be identified as IIR-CR2 which is a multiconductor control line from the top of the mast. The antenna to which it was connected, the SRD-501, was renoved during the test leaving the cable open circuited at the top. The second cable modeled is not 
present on the Huron. However, there is a similar cable which does terminate in EWCR, but it does not have any connections in $\mathrm{CCR}$ as modeled. The measurement for 11R-CR2 is identified as EWCR-02-01 shot no. 1049. It was also taken with a -2 probe.

Communications Control Room (CCR) The cables modeled in CCR are in error. There is no bundle of ten wires from a cable in EWCR. The other cable was run from level J, via a connector, to the AS-5041 antenna. There is no cable connected to either port or starboard AS-5041 which terminates in CCR. For purposes of comparison a similar cable was chosen which runs from the AT-924B antenna. Three cables were measured during the test. Two of these are RF cables, R-RA20 and R-RA22, and one is a control cable, R-RAC5. The measurements are identified as CCR-06-01 shot no. 917, CCR-08-01 shot no. 888, CCR11-02 shot no. 637 .

Radar and TACAN Room (RTR) One modeled cable runs from the TACAN antenna to RTR. Seven TACAN cables were measured, but only the maximum and minimum measurements are identified: R-BA24, TAC-02-02, shot no. 171 and R-BAIl, TAC05-01, shot no. 137 .

Iransmitter Room Number One (TR-1) The cable modeled runs from one side of the flattops antenna to TXI. The cable measured connects to a junction box on the mast which is fed by both halves of the flattops fan. The measured data are identified as 2R-RAl, LINK-08-01, shot no. 967. These data were measured with a -2 probe. 
Figures A.9 and A.11 compare the time-domain responses of the measured and predicted data at each location followed by Figs. A.10 and A.12, the transforms of the LLL data. The data are analyzed in the same manner as for the mast structural elements.

Table A.4 compares the interior cable responses considered fair. The LLL data were corrected and the NSWC data were obtained with a Singer 51550-2 current probe with a sensitivity of $1 \mathrm{~mA}: 1 \mathrm{mV}$.

\section{A.2.3 ANTENNA CENTER CONDUCTOR RESPONSES}

Antenna center conductor predictions were made by LUL for seven antennas. Table A.5 summarizes the NSWC observations about these msurements. From these observations only the HF transmit whips, HF receive fans, and AT-924B horizontal whip antennas can be compared for $\mathrm{V} \propto \mathrm{c}$ and $\mathrm{I}_{\mathrm{sc}}$ ** $^{*}$

${ }^{*} I_{50 \Omega}$ only for the horizontal whip.

TABLE A.4. Interior cable data comparisons.

\begin{tabular}{lccccc}
\hline & \multicolumn{2}{c}{ NSWC measurements } & & \multicolumn{2}{c}{ LLL predictions } \\
\cline { 2 - 3 } Location & Freq comp, & Pk ampl, & Freq comp, & pk ampl, \\
\hline EWCR & MHz & A & & MHz & A \\
RTR -no. 137 & $1^{a} 2,6$ & 15 & 2,8 & 17 \\
- no. 171 & $2(?), 4(?)$ & 1.5 & $2(?), 4(?)$ & $24^{\text {b }}$ \\
\hline
\end{tabular}

$a_{A}$ signal of approximately $1 \mathrm{MHz}$ is internally generated by the ship as a reference signal.

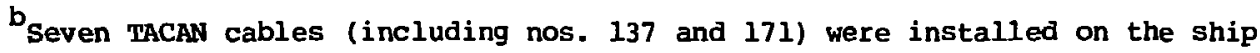
but only one cable was modeled. If the other five TACAN cables carried currents on the same order as for nos. 137 and 171, the total current would be comparable to the predicted current. 
TABLE A.5. NSWC antenna measurement observations.

\begin{tabular}{|c|c|c|}
\hline Antenna & Data photo ID & Comments \\
\hline AS -5058 top mast & $\begin{array}{l}\text { ANT no. I } \\
\text { 3R-RA9, RTR2-04-01 } \\
\text { shot no. 1460 1461 } \\
\text { 3R-RA10, RTR2-05-01 } \\
\text { shot no. 1454 1455 }\end{array}$ & $\begin{array}{l}\text { Data taken at end of } \\
\text { cable, not at base of } \\
\text { antenna. Only normal } \\
\text { mode current measured. }\end{array}$ \\
\hline HF transmit whips & $\begin{array}{l}\text { ANT-0I } \\
V_{O C} \text { shot no. } 1051-1054 \\
I_{s c} \text { shot no. } 841-843 \\
I_{n} \text { shot no. } 848-850\end{array}$ & $\begin{array}{l}\text { Port side; } 5052 \text { current } \\
\text { not measured. In is } \\
\text { normal mode current } \\
\text { into coupler. }\end{array}$ \\
\hline HF receive Eans & $\begin{array}{l}\text { ANT-18 } \\
\mathrm{V}_{\text {OC }} \text { shot no. } 190 \& 198 \\
\mathrm{I}_{\text {sC }} \text { shot no. } 1358-1359\end{array}$ & Aft, stbd side. \\
\hline $\begin{array}{l}\text { AN/SRC- } 23 \text { flat } \\
\text { tops }\end{array}$ & $\begin{array}{l}\text { Mast- } 34 \\
V_{n} \text { port shot no. } 1159 \\
V_{n} \text { stbd shot no. } 1150 \\
I_{n} \text { port shot no. } 1120 \\
I_{n} \text { stbd shot no. } 1121\end{array}$ & $\begin{array}{l}\text { Antenna connected to } \\
\text { coupler. Only normal } \\
\text { voltage and current } \\
\text { measured. }\end{array}$ \\
\hline $\begin{array}{l}\text { AS- } 5048 \\
\text { AS- } 5049 \\
\text { AN/WLR-1C }\end{array}$ & None & $\begin{array}{l}\text { Data measured below in } \\
\text { ENER. Comparison not } \\
\text { possible. Further com- } \\
\text { plication is that the } \\
\text { two UHF antennas are } \\
\text { connected together in } \\
\text { a tee on the mast. }\end{array}$ \\
\hline $\begin{array}{l}\text { AN/SRN- } 12 \\
\text { onega }\end{array}$ & None & No data taken. \\
\hline $\begin{array}{l}\text { AT-924B horiz. } \\
\text { whip }\end{array}$ & $\begin{array}{l}\text { Mast-29 } \\
I_{50} \text { shot no. } 1151 \\
\text { and } 1149\end{array}$ & $\begin{array}{l}\text { Data taken at no. } 4 \\
\text { antenna, port side, top } \\
\text { of mast. }\end{array}$ \\
\hline
\end{tabular}

Figures A.13, A.15, A.17, A.19, and A.21 compare the time-domain responses of the measured and predicted data at each location followed by Figs. A.14, A.16, A.18, A.20, and A.22, the transforms of the LLL data. The data are analyzed in the same manner as for the preceding two categories.

Table A.6 compares the NSWC antenna center conductor responses to the LLL predictions. The NSWC äata have been corrected by a calibration factor of 1000 for Voc, $1 \mathrm{~mA}: 1 \mathrm{mV}$ for $I_{s c}$ and $1 \mathrm{~A}: 5 \mathrm{~V}$ for $I_{50 \Omega}$ and the LuL data have been corrected as before. 
TABLE A.6. Antenna data comparisons.

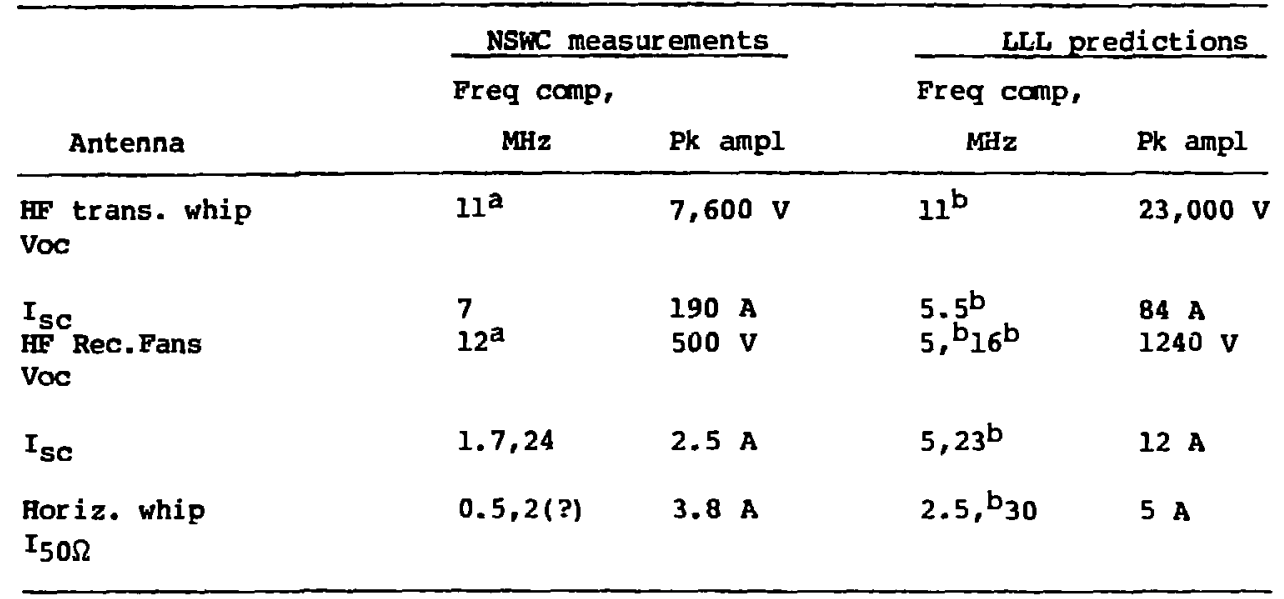

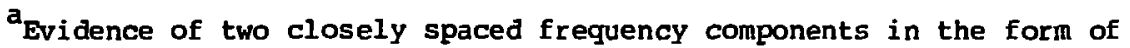
"beating."

$b_{\text {Transform used to help establish resonant frequency component in the }}$ response.
}

\section{A. 3.0 MODEL ASSESSMENT}

The model predictions are modestly encouraging. This overall assessment is based on the following gross features of the model predictions versus measurement:

1. Time-domain response peaks generally agree to within $\pm 6 \mathrm{~dB}$ and only occasionally exceed $\pm 10 \mathrm{~dB}$.

2. A 2-MHz response peak that can be associated with the length of the mast is typically reproduced by the predictions when it is present in the measurements.

3. Some high-frequency components are resolved as in the EWCR interior cable response, the HF transmitter whip responses, and the HF receive fans Isc response.

The following deficiencies were noted in the predictions:

1. LLL overpredicts peak time-domain amplitudes.

2. Responses are overdamped compared to measurements. 
3. Closely spaced resonances in the frequency domain lead to an observed beating phenomena in the measured time-domain response that is not evident in the predicted time-domain response for several data sets.

The measurements themselves were also at times less than ideal as

Fig. A.19 demonstrates, but same allowance is made for this in the assessment of agreement between predictions and measurement.

It would appear that for future model predictions to agree more closely with measurements, extreme care will have to be taken in the model fidelity. Some of the features that are considered critical include: cable rooting, cable size, cable interconnects, types of measurements to be made, room geometry, distance of cable from adjacent walls, and number of cables. This will require a very close interaction among all the test participants to avoid some of the ambiguities apparent in this effort. Further, the verification of the model predictions--which are quite broadband--will require sophisticated test measurements that, via faster sweep speeds and signal conditioning (high pass filtering typically), resolve a data bandwidth commensurate with the predicted data bandwidth. 

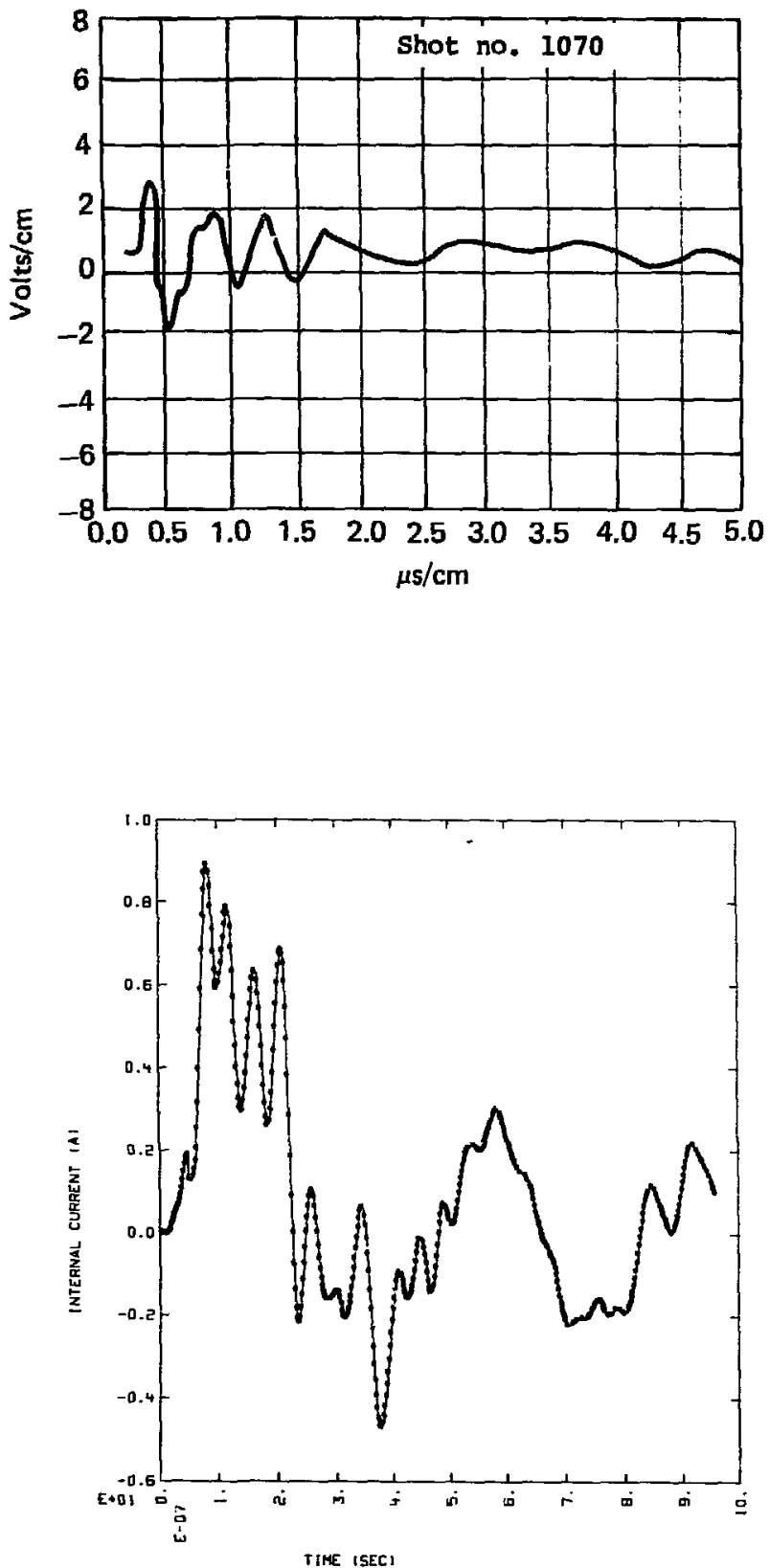

FIG. A.1. Full-scale current on horizontal mast element at level G. A-II 


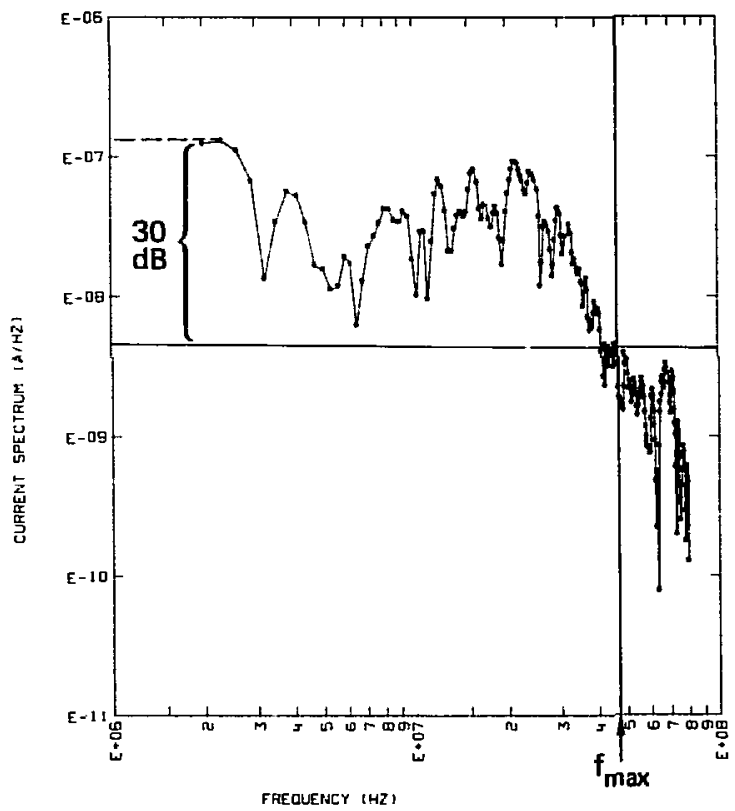

FIG. A.2. Full-scale current frequency spectrum on horizontal mast element at level G. 

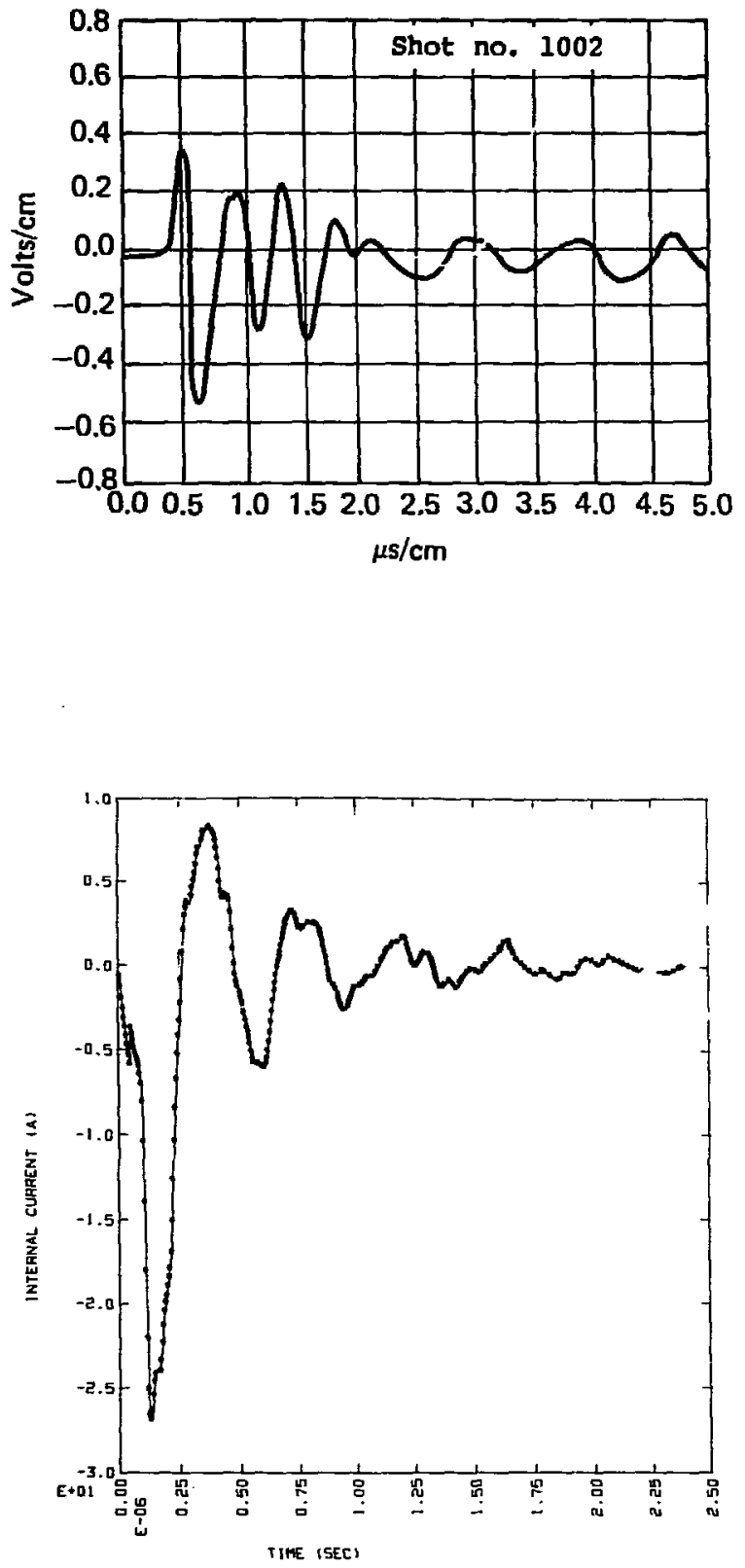

FIG. A.3. Full-scale current on mast between levels $\mathbf{G}$ and $\mathbf{H}$. 


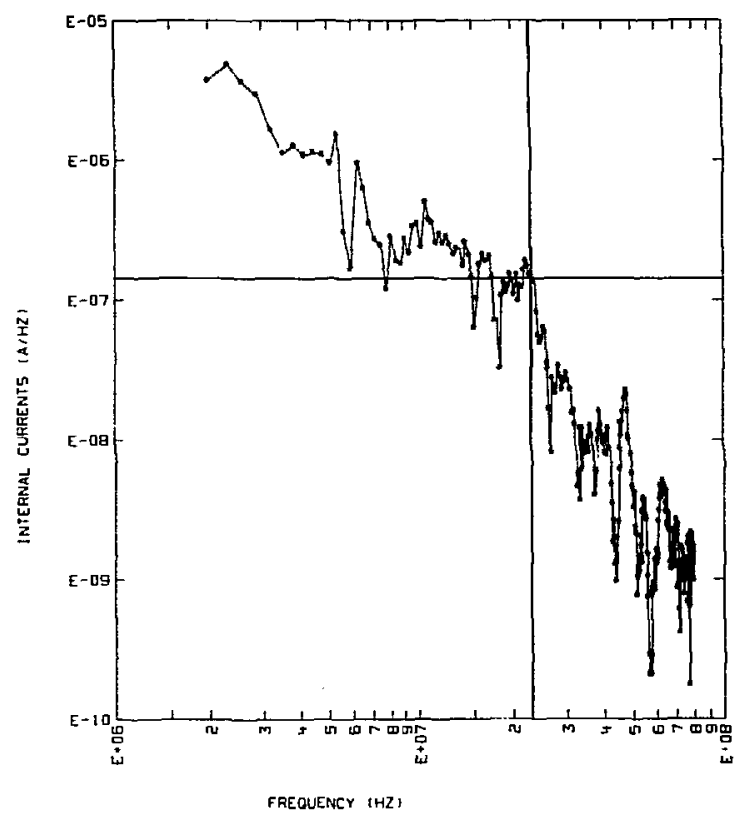

FIG. A.4. Full-scale current frequency spectrum on mast between levels $\mathbf{G}$ and $\mathbf{H}$. 

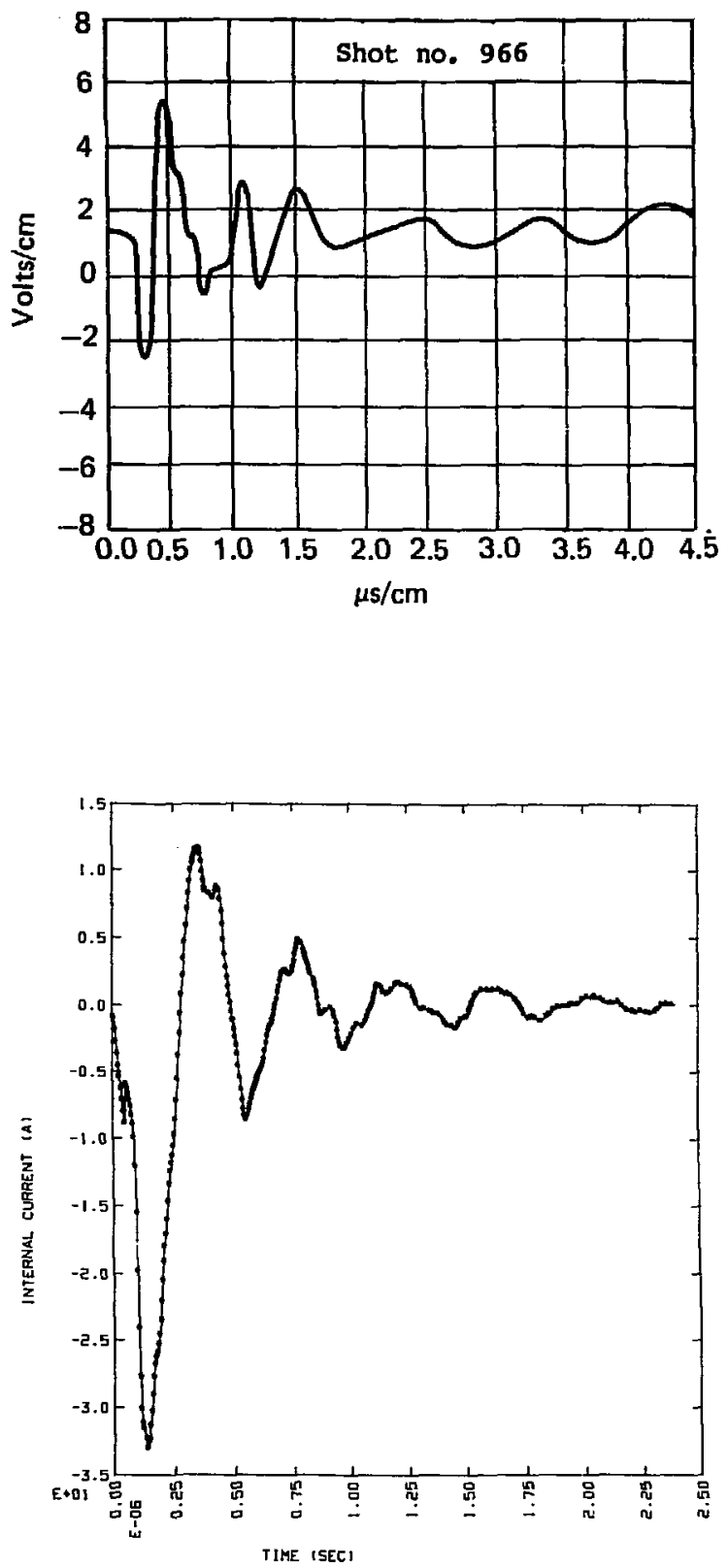

FIG. A.5. Full-scale current on mast between levels $C$ and $D$ (forward port side). A-15 


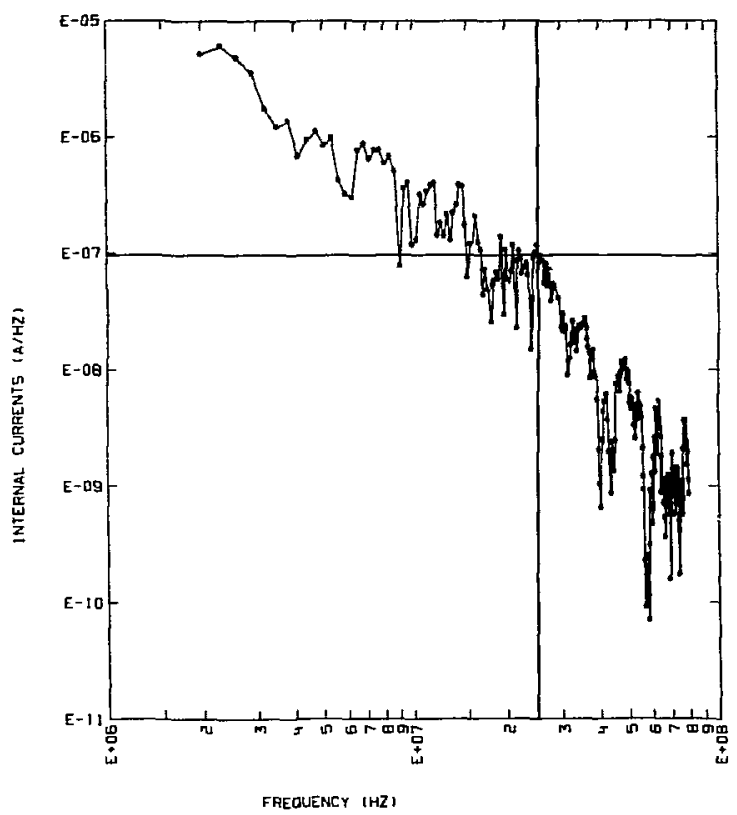

FIG. A.6. Full-scale current frequency spectrum on mast between levels C and D (Eoríara port siaje). 

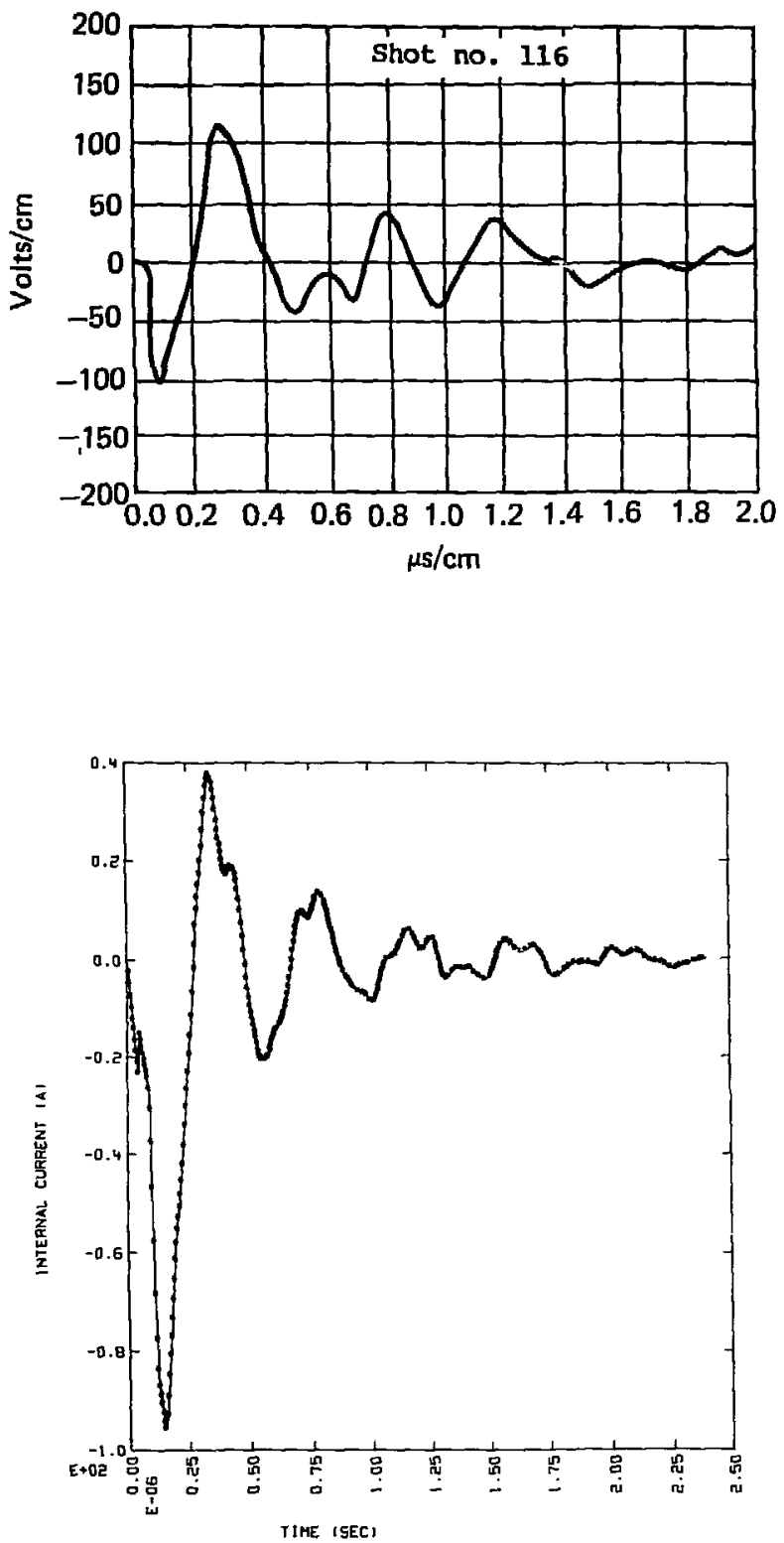

FIG. A.7. Full-scale current on mast between levels $A$ and $B$ (forward port side). 


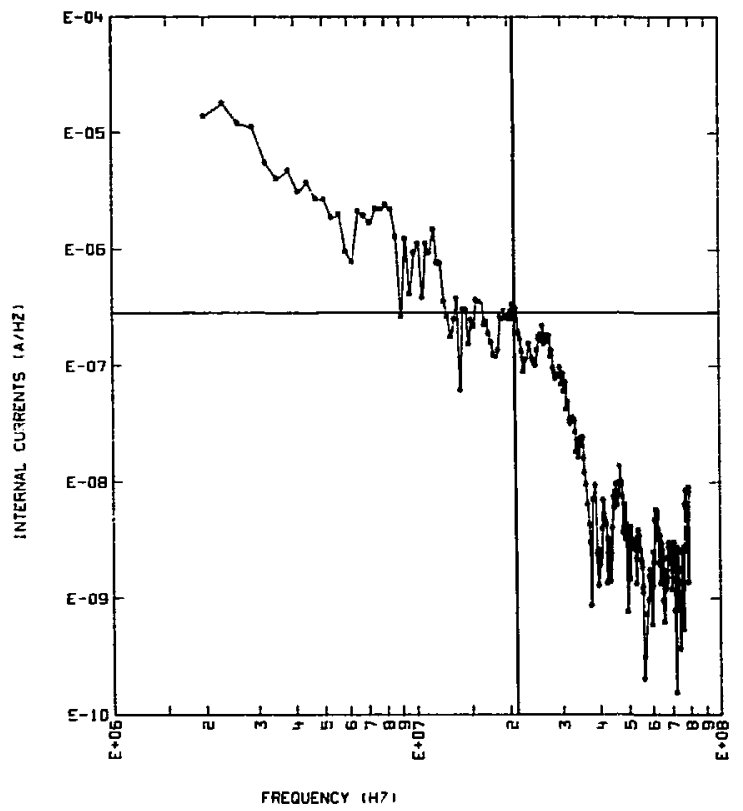

FIG. A.8. Full-scale current spectrum on mast between levels $A$ and B (forward port side). 

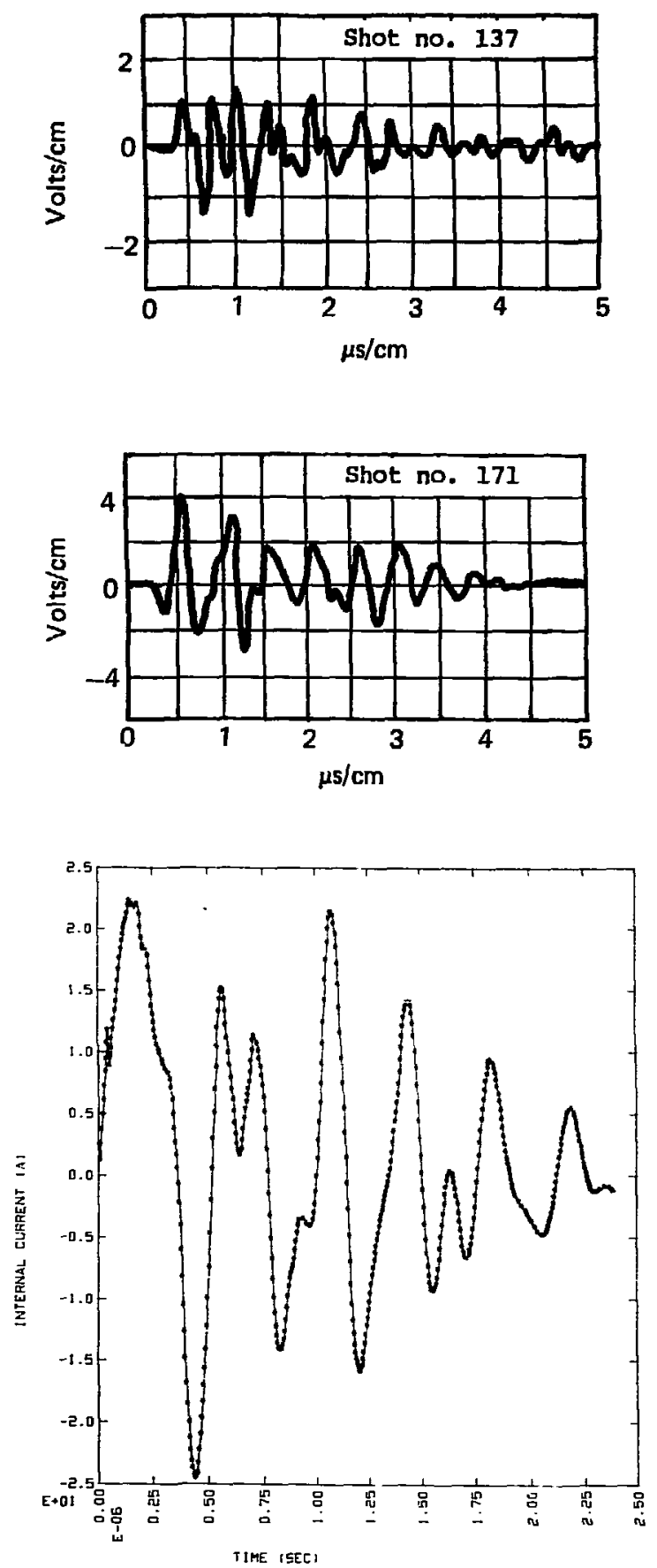

FIG. A.9. Full-scale bulk cable current (TACAN room). 


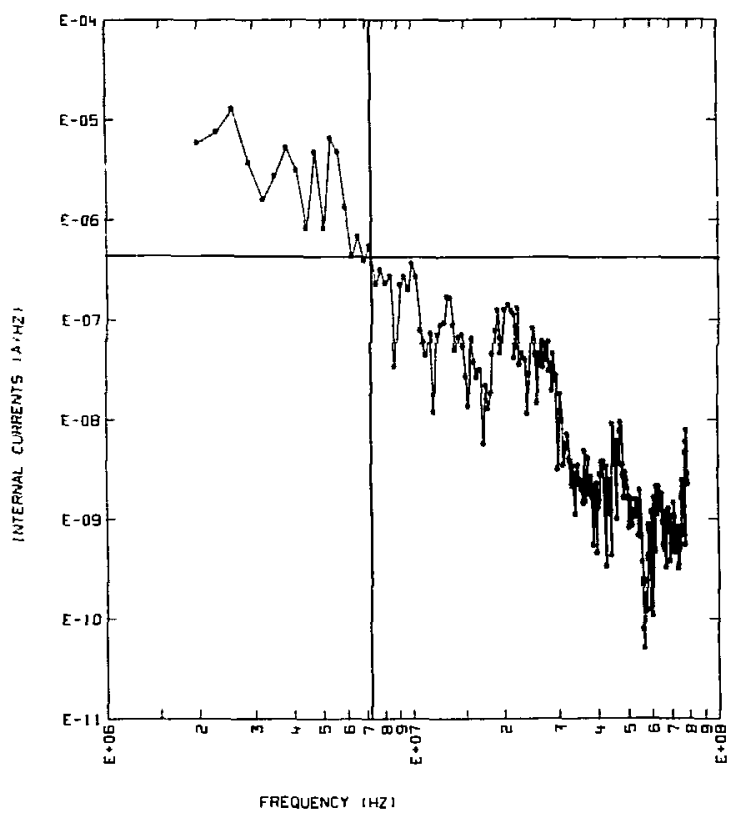

FIG. A.10. Full-scale bulk cable current spectrum (TACAN room) . 

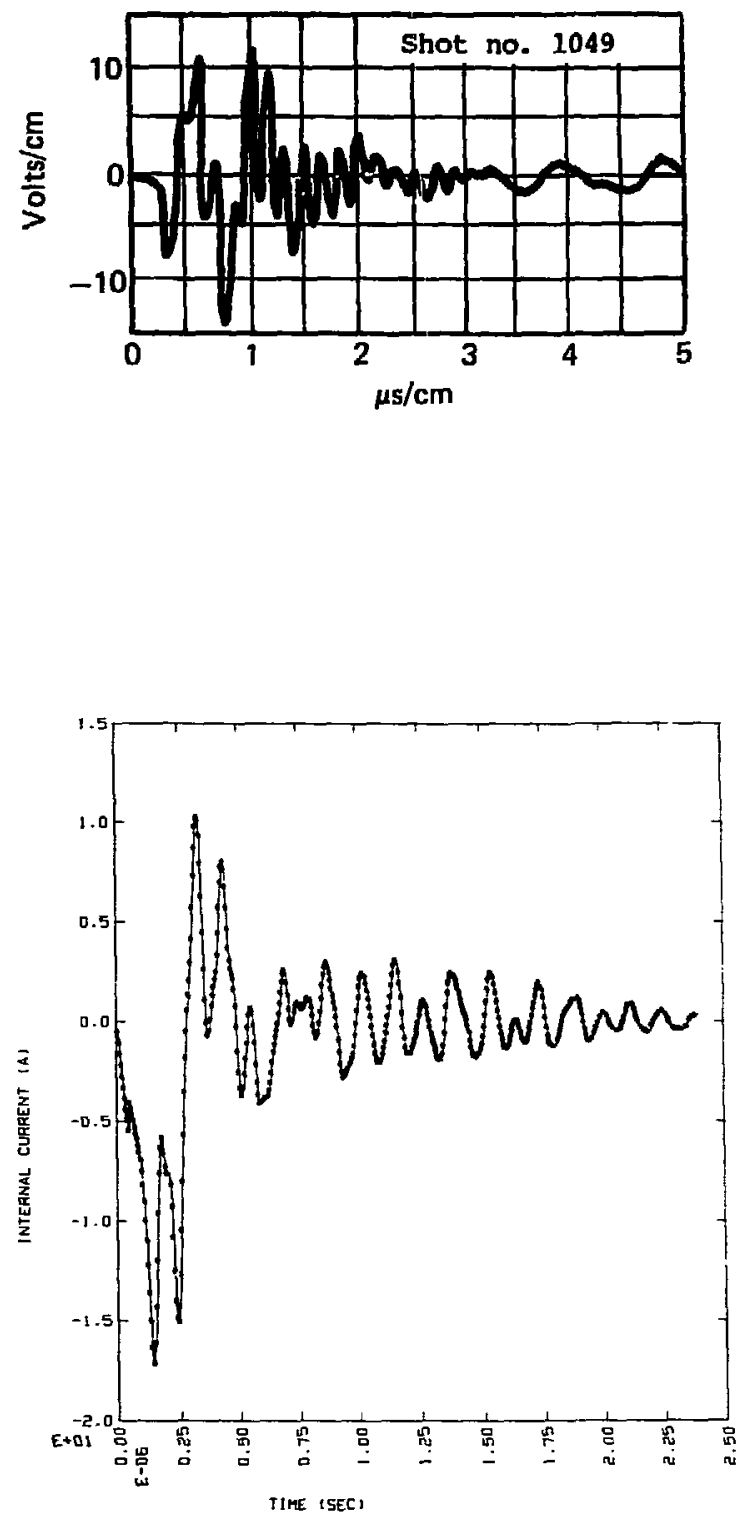

FIG. A.11. Full-scale bulk cable current (EWCR roon). A- 21 


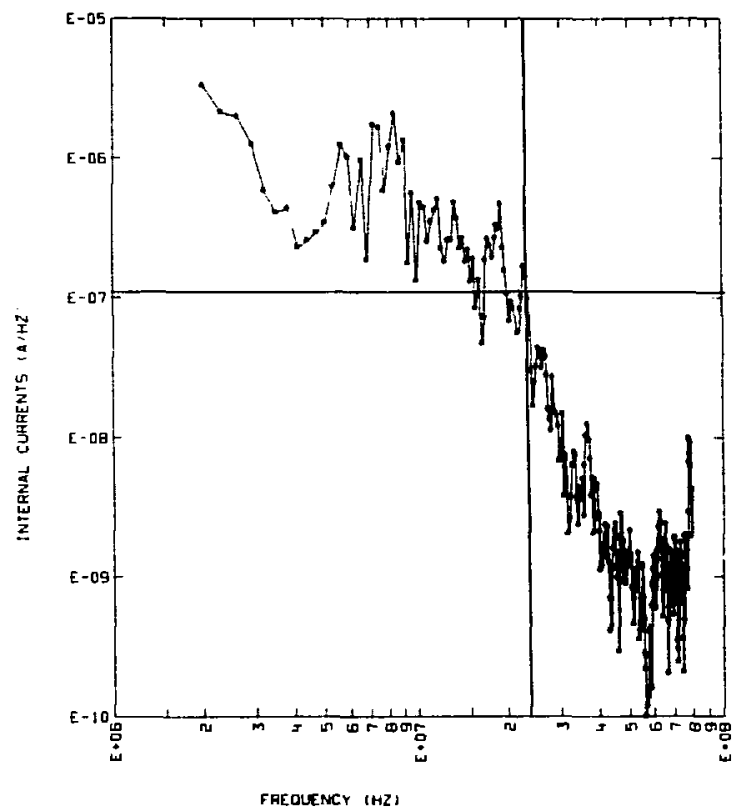

FIG. A.12. Full-scale bulk cable current spectrum (EHCR room). 

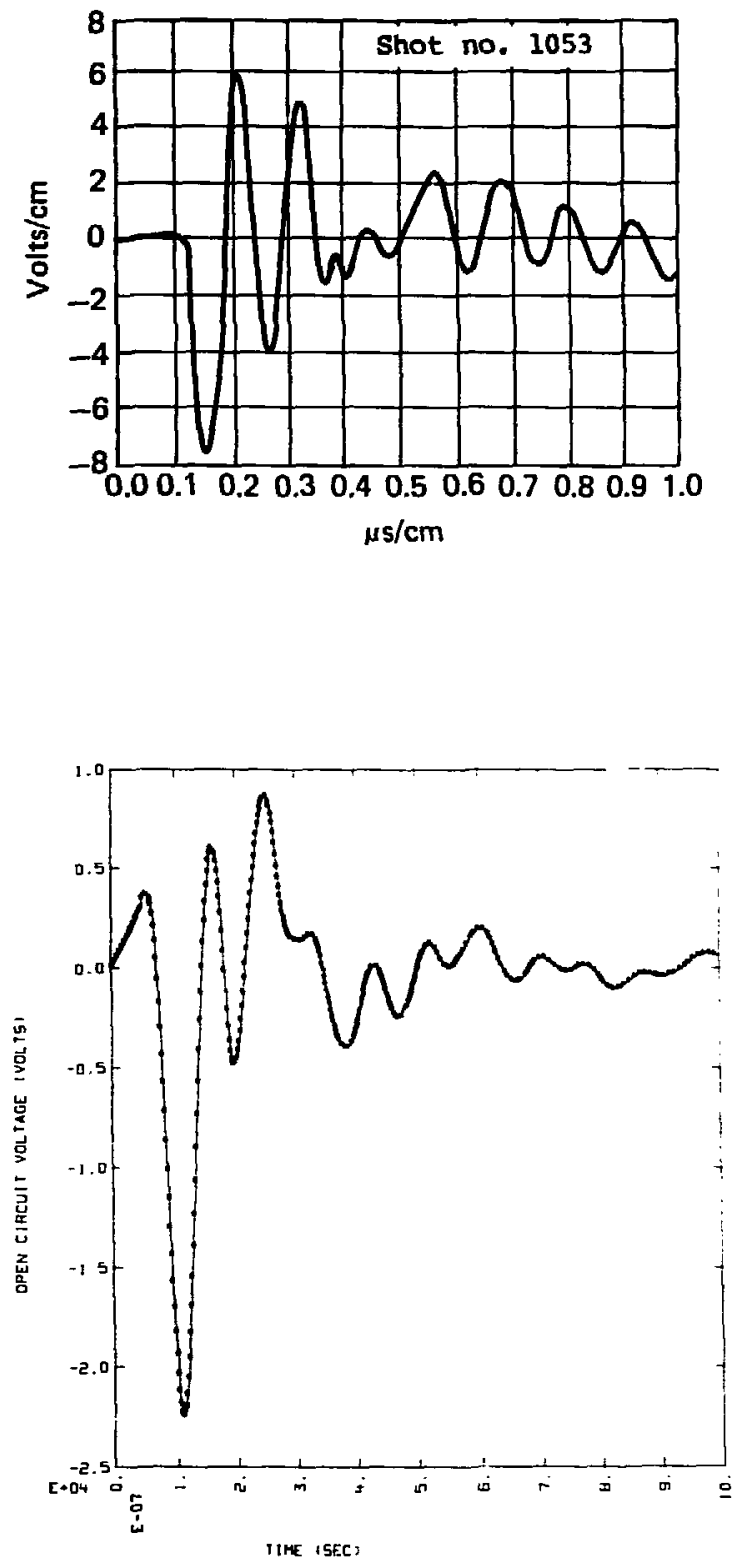

FIG. A.13. Full-scale open-circuit voltage for 35-ft whip antenna (port). 


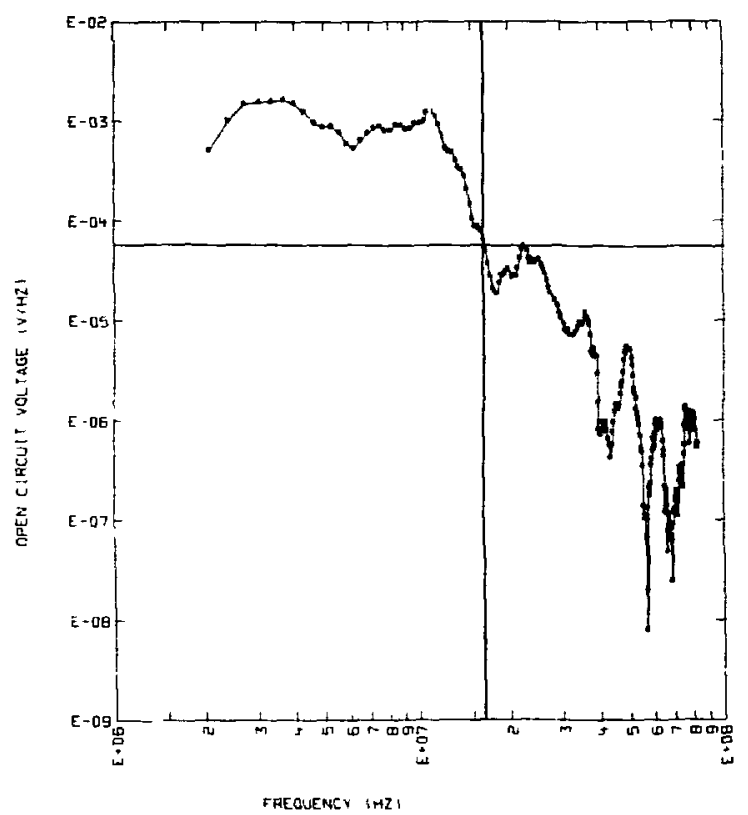

FIG. A.14. Full-scale open-circuit voltage spectrum for 35-ft whip antenna (port). 

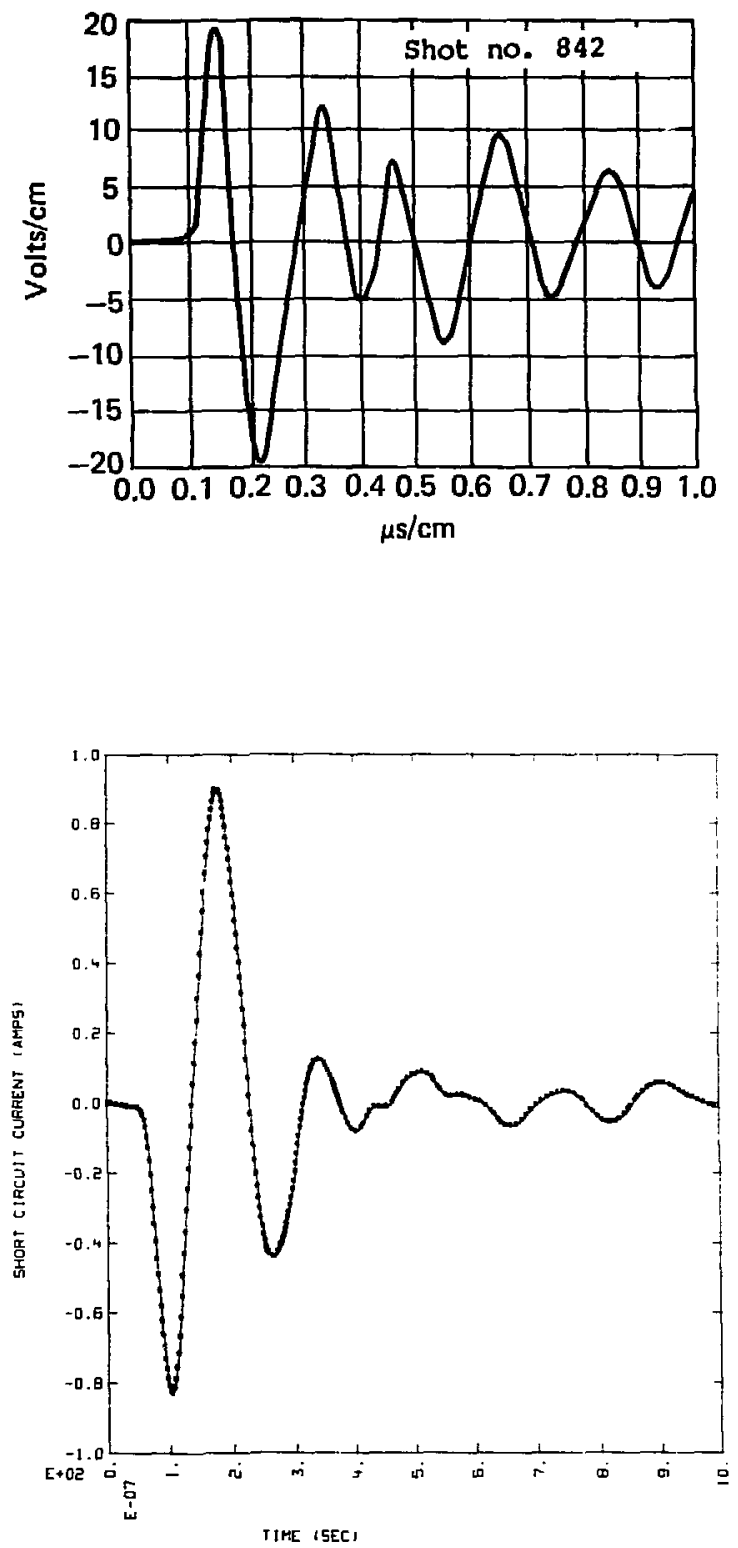

FIG. A.15. Full-scale short-circuit current for 35-ft whip antenna (port). 


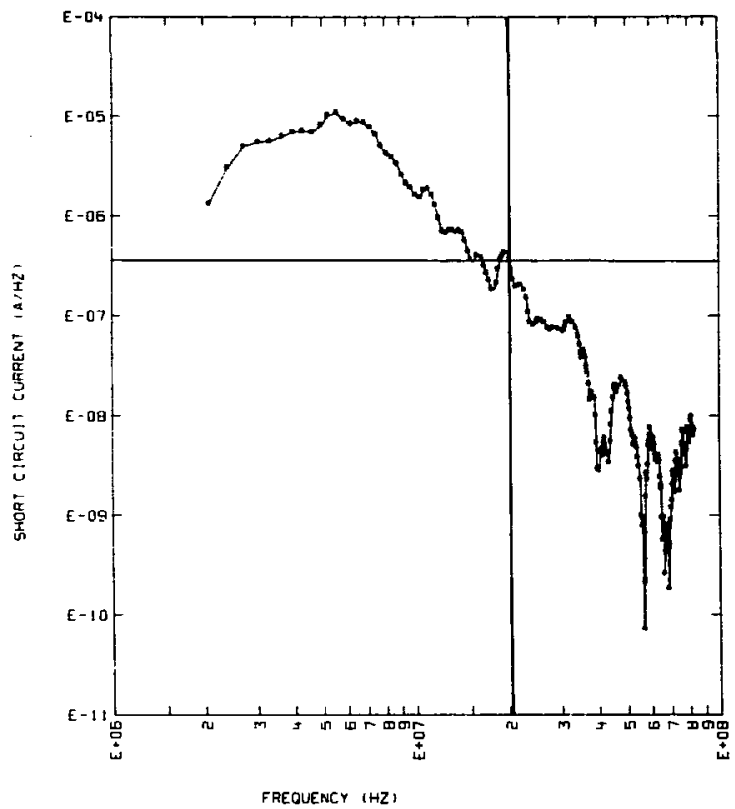

FIG. A.16. Full-scale short circuit current spectrum for 35-ft whip antenna (port). 

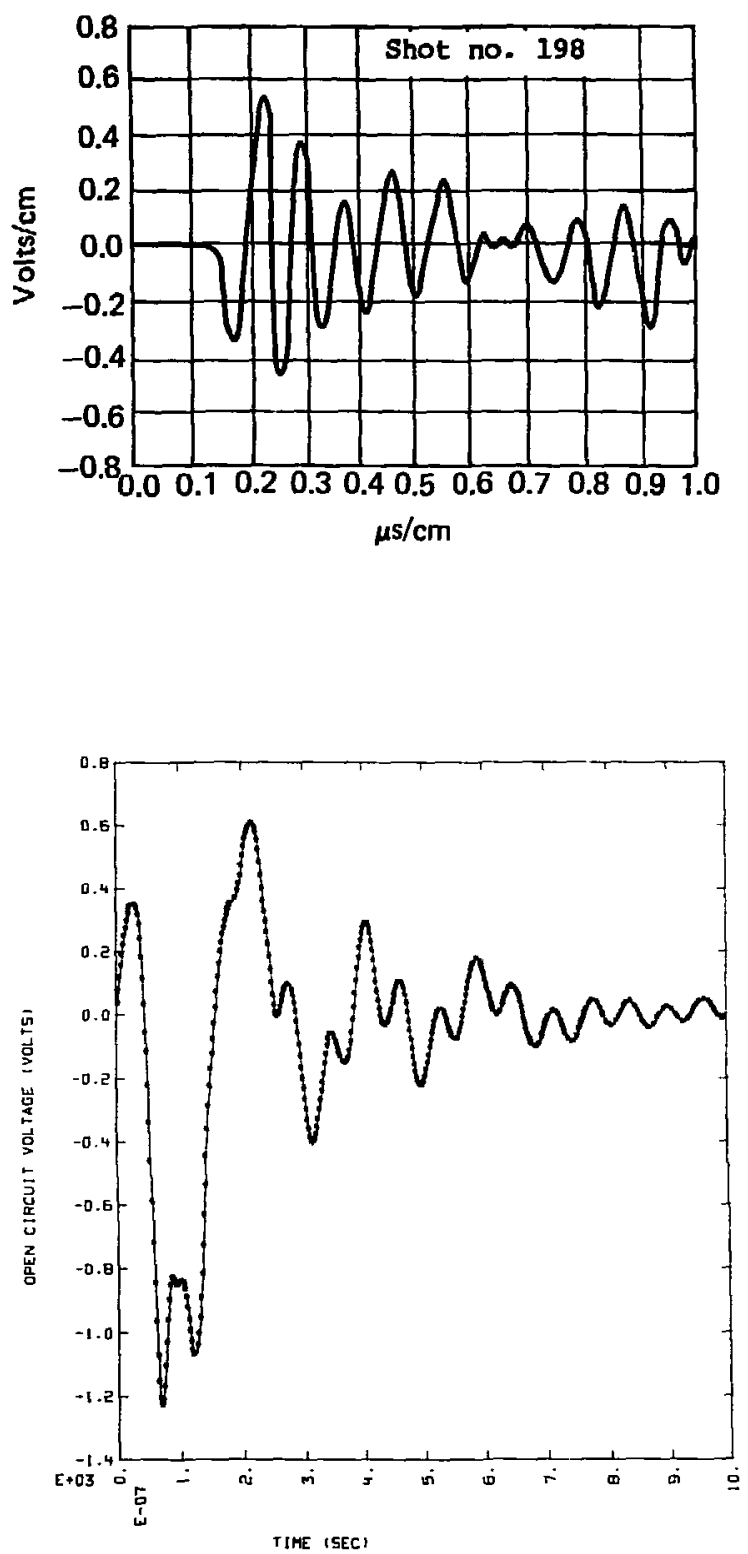

FIG. A.17. Full-scale open-circuit voltage for lower fan antenna (starboard). 


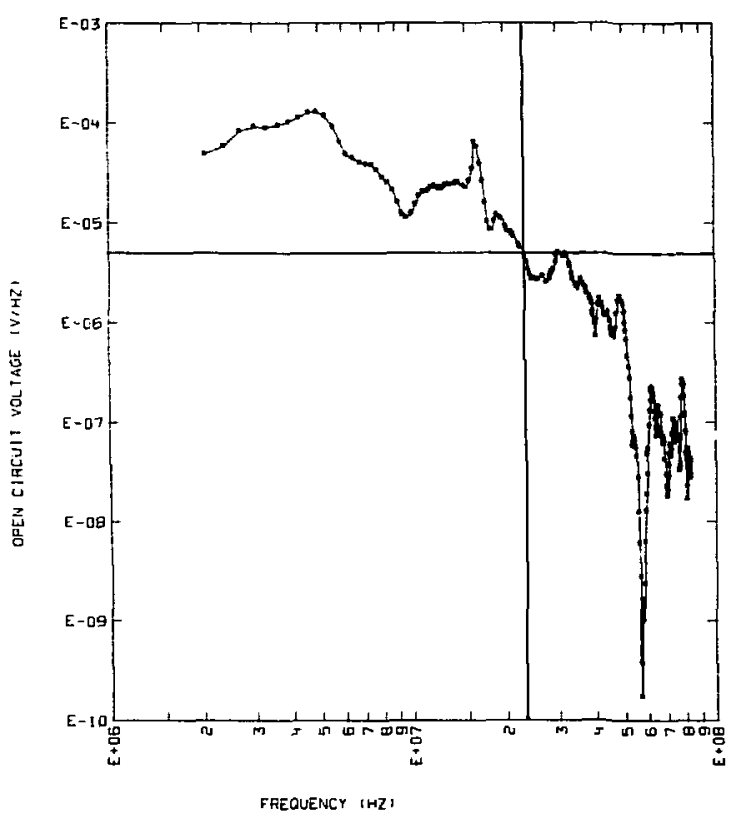

FIG. A.18. Eull-scale open-circuit voltage spectrum for lower fan antenna (starboard). 

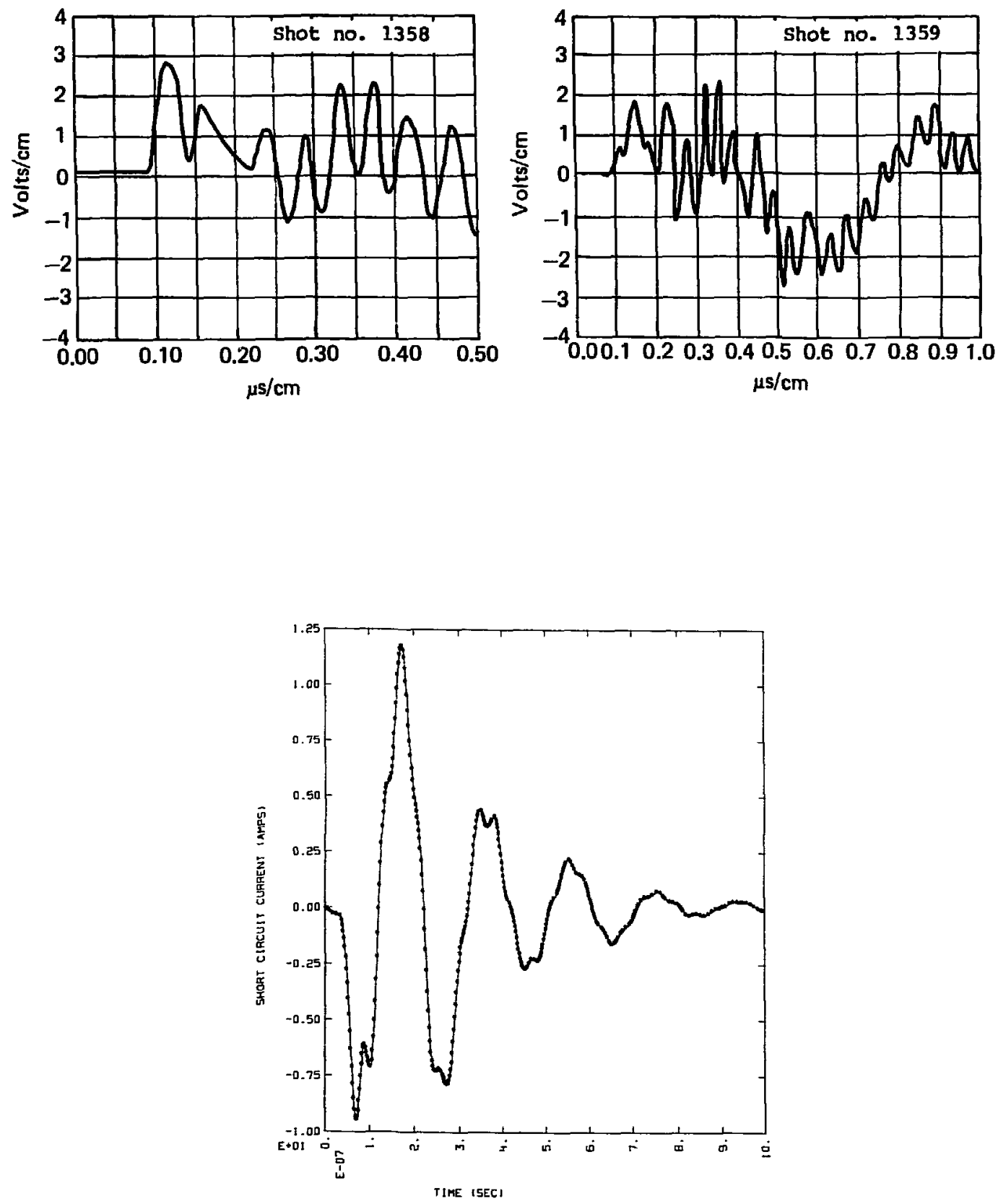

FIG. A.19. Full-scale short-circuit current for lower fan antenna (starboard). 


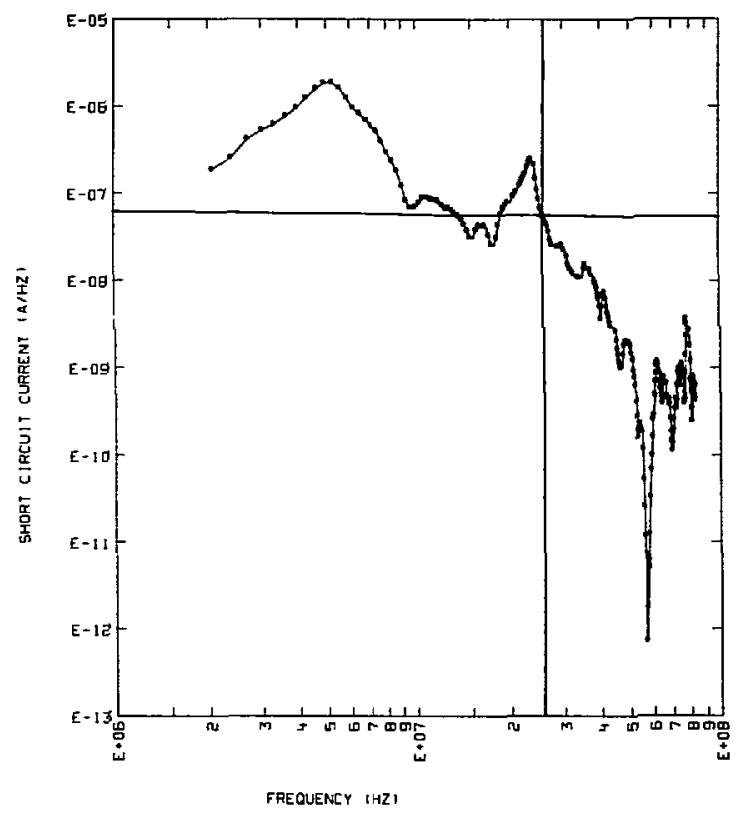

FIG. A.20. Full-scale short-circuit current spectrum for lower fan antenna (starboard). 

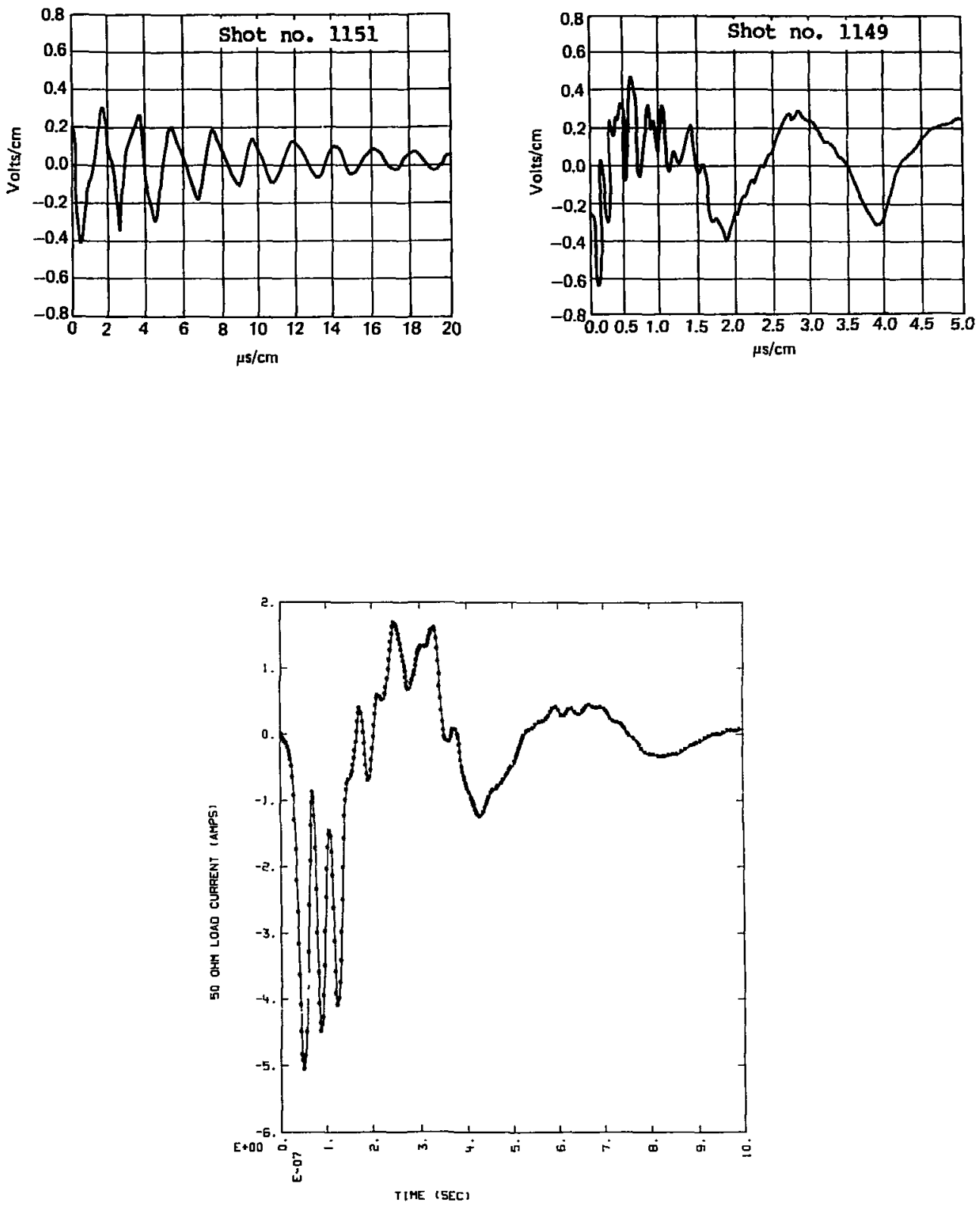

FIG. A.21. Full-scale current for horizontal whip (port side of mast). 


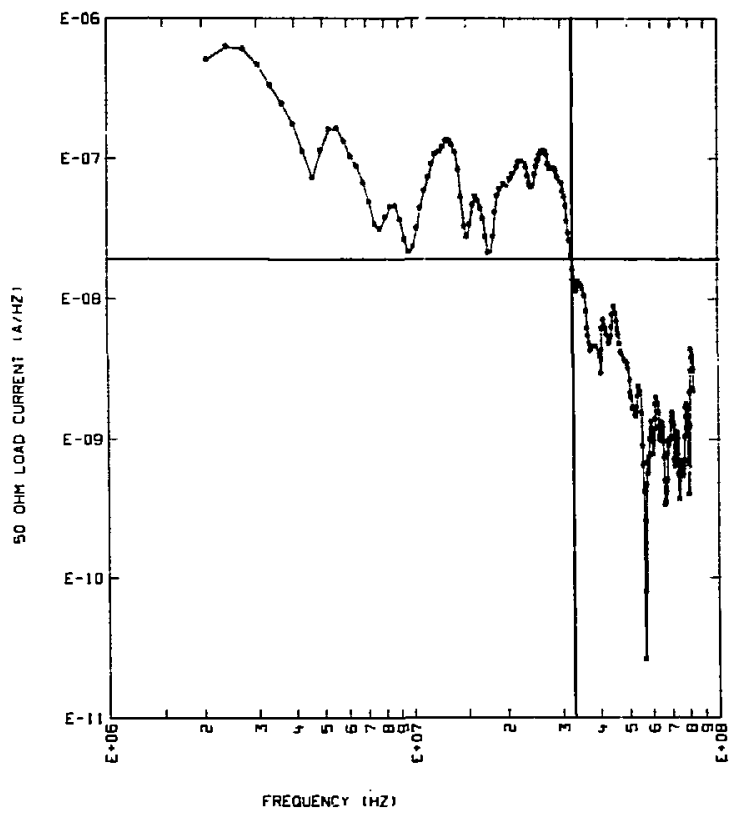

FIG. A.22. Full-scale current spectrum for horizontal whip (port sice of mast). 


\section{REFERENCES}

1. K. S. Kunz and K. M. Lee, "A Three-Dimensional Finite-Difference Solution of the External Response of an Aircraft to a Complex Transient EM Environment: Part 1 - The Method and its Implementation; anā Part 2 - Comparison of Predictions and Measurements," in IEEE Trans. EMC, Vol. EMC-20, No. 2, May 1978.

2. v. Leipa, "Surface Field Measurements on Scale Model EC-135 Aircaft," in Interaction Application Memo 15, AFWL Note Series. Carl Baum, Ed. (August 1977). 